\title{
Doppler studies in the femoro-popliteal pathway
}

Citation for published version (APA):

Bruins Slot, H. (1981). Doppler studies in the femoro-popliteal pathway. [Doctoral Thesis, Maastricht University]. Rijksuniversiteit Limburg. https://doi.org/10.26481/dis.19810626hb

Document status and date:

Published: 01/01/1981

DOI:

10.26481/dis.19810626hb

Document Version:

Publisher's PDF, also known as Version of record

\section{Please check the document version of this publication:}

- A submitted manuscript is the version of the article upon submission and before peer-review. There can be important differences between the submitted version and the official published version of record.

People interested in the research are advised to contact the author for the final version of the publication, or visit the DOI to the publisher's website.

- The final author version and the galley proof are versions of the publication after peer review.

- The final published version features the final layout of the paper including the volume, issue and page numbers.

Link to publication

\footnotetext{
General rights rights.

- You may freely distribute the URL identifying the publication in the public portal. please follow below link for the End User Agreement:

www.umlib.nl/taverne-license

Take down policy

If you believe that this document breaches copyright please contact us at:

repository@maastrichtuniversity.nl

providing details and we will investigate your claim.
}

Copyright and moral rights for the publications made accessible in the public portal are retained by the authors and/or other copyright owners and it is a condition of accessing publications that users recognise and abide by the legal requirements associated with these

- Users may download and print one copy of any publication from the public portal for the purpose of private study or research.

- You may not further distribute the material or use it for any profit-making activity or commercial gain

If the publication is distributed under the terms of Article $25 \mathrm{fa}$ of the Dutch Copyright Act, indicated by the "Taverne" license above, 
Doppler studies in the femoro-popliteal pathway 
The publication of this thesis was supported by the 'Dutch Heart Foundation'.

Het verschijnen van dit proefschrift werd mede mogelijk gemaakt door steun van de Nederlandse Hartstichting. 


\section{Doppler studies}

\section{in the}

\section{femoro-popliteal pathway}

\section{PROEFSCHRIFT}

ter verkrijging van de graad van doctor in de geneeskunde aan de Rijksuniversiteit Limburg te Maastricht op gezag van de rector magnificus Prof. Dr. W.H.F.W. Wijnen, hoogleraar aan de Faculteit der Geneeskunde, volgens besluit van het College van Dekanen, in het openbaar te verdedigen in de aula van de universiteit op vrijdag 26 juni 1981 des namiddags om vier uur.

door

\section{H. Bruins Slot}

geboren te Leiden 
Promotores : Prof. Dr. R.S. Reneman, Maastricht Prof. Dr. J.M. Greep, Maastricht

Referenten : Prof. D.E. Strandness, Jr., M.D., Seattle, U.S.A. Prof. R.G. Gosling, Ph.D., London, U.K.

Prof. Dr. H.A.J. Lemmens, Maastricht 
To Bartje, Annemaria and their wonderfull mother Liesbet 
This study was conducted in the vascular laboratory and the department of surgery of the St. Annadal hospital and the
University of Maastricht. 


\section{Contents}

Preface

Chapter 1 Introduction: the surgical perspective of hemodynamic studies 11

Chapter 2 Parameters of vascular disease: a concise review of the literature 16 2.1 Introduction

2.2 The radiological visualization of blood vessels 16

$\begin{array}{ll}2.3 \text { Volume flow and arterial pressure } & 17\end{array}$

2.4 The noninvasive measurements of arterial pressure 18

2.5 The velocity wave form and methods for its quantification 19

2.6 The measurement of transit-time 21

2.7 Doppler velocity meters: principle 22

2.8 The arterial velocity profile and the Doppler frequency spectrum

2.9 Continuous wave and pulsed Doppler instruments 23

2.10 Single-gate and multi-gate pulsed Doppler instruments 24

2.1. Methods of signal processing 24

2.12 Pulsatility index, transit-time and the Doppler velocity meter

2.13 Aim of the present study

Chapter 3 Materials and Methods

3.1 Introduction

3.2 The patients

3.3 Arteriography

3.4 The instrumentation

3.5 Examination of the patient

3.6 Off-line data processing definitions

3.7 Sources of error and inaccuracy 45

3.7.1 Introduction

45

3.7.2 The signal-to-noise ratio 45

3.7.3 The wave form analyser 45

3.7.4 The pulse repetition frequency 47

3.7.5 Patient- and examiner-related sources of inaccuracy

3.7.6 The cumulative effect from all sources of error and inaccuracy

3.8 Statistical methods

3.8.1 Discriminant analysis

3.8.2. Other statistical tools 
4.1 Introduction

4.2 Comparison of PI and DT peak-to-peak obtained with both Doppler instrumentations

4.2.1 Results

4.2.2 Discussion

4.3 The ultrasonic estimation of the arterial diameter

4.3.1 Results

4.3.2 Discussion

4.4 The ankle systolic pressure index

61

4.4.1 Results

4.4.2 Discussion

61

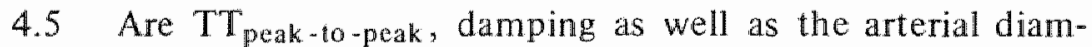
eter of the SFA, as determined in the femoro-popliteal pathway dependent on aorto-iliac lesions?

4.5.1 Results

4.5.2 Discussion

4.6 Is the velocity signal in the common femoral artery influenced by distal atherosclerotic lesions?

4.6.1 Results

4.6.2 Discussion

4.7 The hemodynamic parameters in rellation to femoro-popliteal disease

4.7.1 Results

4.7.2 Discussion

4.8 The effect of age and blood pressure on $\mathrm{TT}_{\text {peak -to-peak }}$ and damping

4.8.1 Results

4.8.2 Discussion

4.9 The discriminative value of the hemodynamic parameters for three arteriographic groups in the femoro-popliteal pathway

4.9.1 Results

4.9.1.1 An example

4.9.1.2 The effect of changes in the a priori classification

4.9.1.3 Discriminant analysis with different parameter combinations

4.9.1.4 Full-length occlusion of the SFA

4.9.1.5 Validation of discriminant analysis

4.9.2 Discussion

Chapter 5. 5.1 General discussion and conclusions

\subsection{Future work}

\section{Summary}

Samenvatting 


\section{Preface}

'A friend and colleague of mine once said that to prescribe sophisticated studies to affirm the diagnosis of an abdominal aneurysm in a patient with a pulsatile mass is as necessary as to perform chromosome tests on a 12-foot truncated pachyderm just to prove it is an elephant'

Francis Robicsek, Editorial Surgery 1.981

This humorous sentiment is not without relevance when applied to the role of modern hemodynamic thinking in vascular surgery. The clinical diagnosis of a failing peripheral vascular system is usually not difficult to make and its surgical treatment, through trial and error, seems well established at present.

Few are the tools, however, which enable the surgeon or physician to anchor his therapeutic measures to fundamental scientific reasoning.

This has inspired a multidisciplinary study to popularize but also to examine critically, a method originally introduced by Gosling in 1969. It is believed by the author that close cooperation between the scientist and the clinician in charge of the patient, is prerequisite for safe advance of the empirical and observational clinician on his way towards new concepts in the treatment of patients with an ailing vascular system. 


\section{LIST OF ABBREVIATIONS}

DT : Delay-time

TT : Transit-time

PI : Pulsatility index

$\Delta \quad$ : Damping

ASPI : Ankle systolic pressure index

CW : Continuous wave

PD : Pulsed Doppler

PRF : Pulse repetition frequency

PWV : Pulse wave velocity

Al : Aorto-iliac

SFA : Superficial femoral artery

pop : Popliteal artery

prof : Deep femoral artery

10 


\section{Introduction: the surgical perspective of hemodynamic studies}

The purpose of this introduction is to bridge the gap seemingly present between day-to-day practice of vascular surgery and the intended evaluation of a practical noninvasive method designed for the hemodynamic description of atherosclerotic lesions in the femoro-popliteal arterial pathway. Increased application of modern technology in the biological sciences added to one's understanding of a number of physiological and pathophysiological phenomena associated with the cardiovascular system. Advanced technology and modern physiological concepts are gradually becoming part of clinical practice in vascular surgery as is apparent from the many recent additions to the medical literature in this field and the rapidly growing number of vascular laboratories.

It seems that traditional thinking is increasingly challenged by new hemodynamic concepts, stimulating discussions on a variety of clinical entities like the role of arteriography in vascular surgery (160) and the feasibility of surgical treatment of occlusive arterial disease $(51)$. The satisfaction derived from difficult surgical accomplishments is moving away from the technical performance itself towards the functional effects of the operation. The therapeutic certainty of vascular reconstructive surgery is gradually replaced by intellectually tormenting questions regarding its usefulness, which does not make surgical life easier. To some extent this gradual change in the approach to the patient with symptomatic arterial disease can be noticed even in the titles of some recent comprehensive textbooks $(72,132,149)$.

The evolution in vascular surgical thinking seems a nalogous to the conceptual changes which have taken place in gastric surgery. No longer are the pro's and cons of different types of gastric resections and anastomoses a subject for lengthy discussion, the emphasis now being placed more on gastric physiology, disturbances of normal physiology and their logical operative sequelae in patients with peptic ulcer disease. This swing from 'how' surgery to "why" surgery becomes also apparent in vascular reconstructive surgery, as is illustrated by the fast growth of the literature dealing with clinical peripheral hemodynamics.

After the classic paper of Linton and Darling in 1962 (91) on femoro-popliteal bypassses, much thas been said on the long term beneficial effect of these reconstructions. The 5-years patency rate of venous bypasses in this region varies with the author from $47 \%$ (2) to $78.9 \%$ (82). Thompson (156) defends his aggressive approach to femoro-popliteal reconstruction with the statement that limb preservation exceeds the patency rate of these bypasses. Strandness and Summer (149) attribute the improvement experienced by patients with an occluded bypass to unconscious adjustment in their life style and to the "powerful psychological effect" of an operation. It is almost needless to say that this will not have been the intention of the operating surgeon.

A fact is that the results of peripheral vascular reconstructive surgery. as given 
by the vast number of papers dealing with this subject, are conflicting from nearly all points of view. Positive and negative correlations of the patency rate of femoro-popliteal bypasses have been noted with diabetes $(104,121,146)$, age $(24,41$, $120)$, angiographic outflow $(32,41,83,120)$, clinical stage of the disease (104, $146)$ and intraoperative graft flows $(12,43)$. Clearly the existing and time-honoured factors suggesting a favourable or unfavourable prognosis fail to be of predictive value in determining the likelihood of early graft thrombosis and failure of revascularisation of the limb in the individual patient.

Femoro-popliteal and femoro-crural reconstructions are generally accepted as limb-saving procedures for patients whose limbs are in jeopardy. Atherosclerotic disease in arteries of the lower extremity usually follows a benign course (101, 168) so that the question, whether relief from claudication in the majority of the patients with intermittent claudication after femoro-popliteal reconstructions outweighs the risk of amputation (1-3\%) after some of the unsuccesful operations, determines a different look on the feasibility of reconstructive surgery in this group of patients. This dilemma is recognized by most vascular surgeons $(2,32$, $41,100,136,171)$ and in fact may contribute to changes in their view regarding the management of patients with intermittent claudication (171). The general opinion is that the risk of losing a limb after reconstruction for claudication is very small $(2,32)$, but also that the beneficial effect of a successful operation cannot be guaranteed for an indefinite period owing to distal progression of atherosclerotic disease (109) or to degenerative changes in the bypass. (152).

Although it is difficult to proclaim an operation 'unnecessary' with certainty, because of the 'semi-exact scientific' nature of medicine, one wonders why femoro-popliteal reconstruction in patients with intermittent claudication is not discussed by Rutkow and Zuidema (133) in their paper on 'unnecessary surgery'. According to the criteria set by the American College of Surgeons, femoro-popliteal reconstruction in patients with intermittent claudication as the sole symptom of their disease, might qualify as a 'comfort operation to alleviate endurable or tolerable symptoms' (133). To rephrase a remark made earlier in this introduction: one knows how to operate on patients with femoro-popliteal disease, but one is less confident in determining the right moment for doing so.

This uncertainty stems from insufficient insight into the progression and regression of atheroselerotic disease in man. Many experiments and observations ( 38 , $61,108,130,145$ ) point at chemical physical or hemodynamic causes for the development of atherosclerosis, which have in common injury of the endothelial cell as the initiating moment for the genesis of atherosclerotic plaques. Surprisingly little emphasis, however, has been placed in surgical literature on trauma to the endothelial cell unwittingly induced by surgical procedures (45). Recent studies demonstrated trauma-provoked atheroma after the use of arterial clamps or embolectomy catheters and following the preparation of vein grafts $(38,152)$. The development of exuberant pannus at arterial anastomotic sites may be the response of endothelium and smooth muscle cells to surgical handling of the vascular tissue (47). The well-known observation that bifurcations are prone to the development of atherosclerotic lesions is thought by some to be associated with higher shear-stress (61) or local turbulence (167) leading to injury of the endothelial cell. That pulsatile flow under physiological conditions may be instrumental to this process $(59,152)$ agrees with repeated observations that the distal arterial system may be protected from atherosclerotic disease by a more 
proximally located occlusion (88). In $30 \%$ of 62 limbs, progression of atherosclerotic disease after femoro-popliteal reconstructions could be demonstrated (109) Hemodynamic quantification of these seemingly pertinent observations is necessary to acquire more insight into the importance of surgery-associated trauma to the development of atherosclerosis.

The follow-up of patients, operated or not, is valuable for correct appreciation of the results of vascular surgery and different regimens of conservative treatment. However, a population selected in this way is less suitable for epidemiological and natural history studies because many opposing or interrelated forces are at work. Symptoms associated with arterial disease may change with time (100), since they are the functional result of progression of the disease and the development of collateral pathways around the site of resistance to flow. For many years surgical interest has been mainly directed to the rapidly expanding technical possibilities in vascular surgery. Little room was left for conservatism in the treatment of intermittent claudication, so appealing was the concept that arteries could be reconstructed (44). With the technical limits explored, interest in the natural history of atherosclerosis is returning (51), stimulated by new concepts regarding its aetiology and pathophysiology.

Widmer (168) conducted an epidemiological survey, rare in its kind, in 6400 factory workers to investigate the incidence of atherosclerotic disease and intermittent claudication in a random, unselected population. Arteriographic evidence of arterial occlusions was present in 99 individuals of whom two-thirds were asymptomatic. The question whether an altered lifestyle or an enormously increased collateral circulation had rendered these patients asymptomatic could not be answered by this study, because no quantifying methods for the measurement of loss of function were available at that time.

More is known about the course of the disease in patients who experience symptoms $(22,78,97,100,115,164)$. There is general agreement on the high mortality from cerebrovascular and coronary heart disease, although reported death rates vary from $3.5 \%$ to $9.5 \%$ annually. Spontaneous improvement in patients with occlusions of the superficial femoral artery is reported in $24 \%$ to $52 \%$ of the cases (101) when relying on the symptomatology of the patient. The many obstacles on the road to better insight into the natural history of the disease include the generally unsystematic scientific approach to the problem, different lengths and ways of follow-up and differences in the composition of studied patient populations. To this may be added the effect of inconsistencies between subjective and objective criteria, i.e. between the patient"s and the examiner's perceptions of the extent of functional impairment.

The measured walking distance on a treadmill and the ankle resting pressure index in two series of patients with intermittent claudication showed poor correlation ( $R$ resp. 0.39 and 0.45 ), although the author's interpretation was quite different $(1,110)$. Extrapolation of these results to the patient's estimation of his experienced limitation in walking activity reduces the diagnostic value of the claudication distance as a functional measure of the disease. This opinion is shared by Verstraete (161) who warns against the use of "purely subjective factors such as intermittent claudication' in the clinical evaluation of drugs recommended for its relief.

In some reports arteriography was used to study the progression of the athero- 
sclerotic lesions. Kuthan (88) studied 1200 limbs with an interval of 2 - 3 years. Fifty-two percent of the patients showed progression of the disease in the superficial femoral artery while the profunda was affected least. Although spontaneous regression of atherosclerotic lesions was observed occasionally (88), improvement of claudication is more likely to occur from development of collateral systems than from disappearance of occlusive lesions.

Two types of collateral pathways in the upper leg may be distinguished. Collaterals can bypass short occlusions in the superficial femoral artery, originating and terminating in this artery. More important, however, is the collateral capacity of the deep femoral artery (162) feeding an extensive network of collaterals which conducts blood to the distal superficial femoral artery or the popliteal artery through one or more re-entry vessels. The efficiency of this collective collateral network is fully dependent on the functional capacity of the deep femoral artery and its main branches, as well as on the collateral network converging on the superficial femoral and/or popliteal artery. Every link in this collateral chain is equally important. From the large accumulated experience with profunda-revascularisation in patients with multilevel disease, aorto-bi-femoral bypasses are known $(28,131,166)$ to have high 5 - and 10-years patency rates owing to the capability of the deep femoral artery to accomodate large flows (16).

Satisfactory clinical results of smaller operations, restricted to the deep femoral artery itself, profundaplasty, were obtained in $20 \%$ to $60 \%$ of the cases $(18,96$, $163,165)$.

Large differences are also reported between the long-term survival rates $(74$, $105)$ and the post-operative increase of the ankle systolic pressure index of patients in different studies $(55,74,90,163)$. Undoubtedly instrumental to these large differences is the radiological inaccessibility of the profunda bifurcation (14, $95,155)$ which has led some surgeons (163) to perform profundaplasty on all patients with rest pain regardless of the radiological appearance of the profunda bifurcation. That indiscriminate use of profundaplasty must lead to unsatisfactory results, is the natural consequence of the already formulated logic that the collateral capacity in the upper leg is as strong as its weakest link. Collaterals and rewentry vessels escape quantitative judgement (31), although some relationship between the clinical response to profundaplasty and the arteriographic appearance of the popliteal artery and the re-entry vessels has been demonstrated by (42).

Anatomy and function are, of course, related, but this does not imply that the radiological assessment of the femoro-popliteal artery is synonymous with the estimation of its function. Clearly, a method is needed which helps the surgeon in his decision if and where to operate on patients with femoro-popliteal disease. The remark has been made that, with regard to femoro-popliteal disease, there are as many radiological classes as there are patients (31). Grading of lesions in this area based on arteriographic information is therefore liable to result in considerable oversimplification.

Diagnostic differentation between patients with well established collateral systems who may eventually become asymptomatic with time and patients who will experience aggravation of atherosclerotic disease with threatening limb loss, may become important in the future philosophy of vascular surgery. Obviously a sensitive method, preferably noninvasive, is needed, which should enable the surgeon to quantify function on a continuous scale. It was therefore 
decided to investigate the diagnostic value of a set of hemodynamic parameters, obtained with continuous wave and pulsed Doppler instruments in patients with atherosclerotic disease in the femoro-popliteal pathway. The rationale of the employed methods and materials is developed in chapter 2 and specified in its last section as the "aim of the present study". 


\section{Parameters of vascular disease: a concise review of the literature}

\subsection{Introduction}

The ideal noninvasive method should be sensitive enough to measure relatively small changes in arterial function. It should combine a high degree of specificity for atherosclerotic disease with an acceptable reproducibility to become a practical clinical method. In addition, the method should be easy to use, harmlless to both patient and examiner and preferably inexpensive.

The optimal function of arteries is best described as their ability to conduct blood to tissues in sufficient quantities for their needs at any time at the cost of minimal encrgy losses (116). This definition emphasizes the inseparable nature of vascular anatomy and function, as either intraluminal impediments or vessel wall changes may have an effect on the transport of blood and its efficiency in terms of increased energy losses.

Progress in the study of physiology and pathophysiology is seriously hampered by the difficulty to 'link up' anatomy and function. Mathematical models and animal experiments often used to study the hemodynamic effect of arterial disease, are undoubtedly of great conceptual value. However, the extremely variable nature of atherosclerosis (145) in terms of intravascular geometry and structure of the vessel wall, allows the extrapolation of experimental findings to the human situation only with certain restrictions. In order to establish an irrefutable relationship between anatomy and function, it will be necessary to compare functional data obtained during life to the physical characteristics of the artery determined after death $(116)$, because at present only few reliable methods are available to study these characteristics in vivo. It is obvious that postmortem verification is hardly feasible in clinically oriented physiological studies.

\subsection{The radiological visualization of blood vessels}

Arteriography, an invasive clinical technique generally available for comparative purposes, provides visual information on atherosclerotic lesions encroaching on the arterial lumen, but its ability to supply objective information on the structure of the vessel wall is limited. Irregularly outlined arteries may suggest the presence of atherosclerotic intimal changes, but it does not supply information on the qualities of the other components of the vessel wall.

The introduction by Dos Santos (50) of translumbar aorto-arteriography marked the beginning of an era in which rapid expansion took place in the field of radiodiagnostics. Although it contributed greatly to the development and practice of arterial surgery (44). shortcomings of arteriography were also noted in more than one aspect. Overestimation of disease in the popliteal and lower leg arteries was noted especially in the presence of multilevel disease $(2,86,120)$. The 
existence of a pressure gradient in the aorto-iliac pathway in some patients with intermittent claudication without showing gross abnormalities on the arteriogram $(37,74,92,107)$ has been reported. The underestimation of disease at the profunda bifurcation is also wellknown and attributable to posteriorly located atherosclerotic plaques and to overprojection of the superficial femoral artery (14,95).

Even if technical shortcomings can be eliminated satisfactorily, functional interpretation of observed lesions will remain difficult. From theoretical and experimental studies $(17,175)$ it is generally assumed that flow remains unimpeded if reduction of the vessel diameter is less than $50 \%$, for which reason most clinical studies differentiate between stenoses less or more than $50 \%(39,80)$. Although this simplification may be acceptable for practical clinical purposes, it is not satisfactory if single plane arteriography is used as a standard reference for the evaluation of new methods, because small alterations in the diameter may result in substantial flow pressure changes especially in this critical area of $50 \%$ narrowing $(17,60,175)$.

The best way to test the 'resolution' of arteriographic techniques is through interobserver variability studies $(46,62,176,29)$. These studies showed without exception poor interobserver agreement, especially in the case of stenosis. Although still considered essential prior to vascular surgery, one can have serious doubts on the ability of arteriography to act as the 'Gold Standard' in comparative studies.

\subsection{Volume flow and arterial pressure}

Despite the considerable difficulty regarding the evaluation and verification of any test applied in clinical physiology, some generally accepted concepts on the functioning of the peripheral arterial system have emerged.

Nature has endowed the human body with compensatory mechanisms aimed at the maintenance of capillary flow and thus tissue oxygenation, when the transport arteries are compromised by obstructing lesions. The relationship of total limb blood flow to the pressure difference over an arterial lesion and to the collective resistance of all arterial pathways, including collaterals, has been established by venous occlusion plethysmography (151) and is predictable to some extent by Ohm's Law, in analogy to electrical theory (149).

There is ample evidence $(11,151,173)$ from plethysmographic studies that volume flow in the calf under resting conditions in patients with intermittent claudication is undiminished owing to the efficiency of compensatory mechatnisms. Although a diminished or slow hyperemic response can be expected distal to the level of disease after exercise or ischemia, plethysmography has only limited diagnostic value for the determination of the level and extent of the arterial lesion $(30,151)$. The undiscriminative nature of plethysmographic volume flow measurements and the present technical inability to measure vessel-specific volume flow, has led to diminished interest in this method.

The intimate relationship between volume flow and arterial pressure does not only apply to hemodynamic events in the complex vascular system of an entire limb, but is also relevant to the local effect of narrowing in an arterial segment. It has been known for many years that a substantial decrease in the lumen of a vessel must occur, before a drop in pressure or flow can be measured distal to the narrowed point. This led to the concept of a 'critical stenosis' which 
was defined by May (99) as a stenotic area in which relatively small alterations in the degree of stenosis cause significant alterations in pressure and volume low. Athough May identified flow velocity as an important factor in determining the 'critical' value for a stenosis, explanatory theoretical and experimental data were provided by Berguer and Hwang (17) based on the Bernouilli theorem regarding the conservation of energy. This approach is unsuitable for the description of the various factors playing a part in the extremely complex hemodynamic events in and around an area of arterial narrowing. Young (175) showed that an experimentally determined Poiseuille-type equation, incorporating blood viscosity and density, blood flow velocity (including its wave form) and the stenosis geometry (including the shape, length and degree of narrowing), is able to predict the pressure gradient across a stenosis, excluding situations in which significant collateral flow contributes to the peripheral tissue perfusion. Studies as referred to above, are of importance to the clinical physiologist because they lend theoretical support to the diagnostic sensitivity of arterial pressure measurements while also calling for their cautious interpretation as, for example, in low flow conditions.

\subsection{The noninvasive measurement of arterial pressure}

The systolic arterial blood pressure can be measured noninvasively with plethysmographic devices $(15,169)$ or with Doppler velocity meters of which the latter are by far the simplest and most accurate means $(84,119,144)$. A standard blood pressure cuff, attached to an ordinary blood pressure meter, is wrapped around the extremity and the return of arterial flow is detected by the Doppler instrument when the cuff pressure is slowly released. These measurements can be carried out at different levels $(3,23,31,60)$ in the lower limb, or just at the level of the ankle, in which case some kind of stress testing (e.g. treadmill exercise or occlusion ischemia) is usually added $(35,147,174)$. To account for time-related variations in the systemic blood pressure (158), especially when treadmill exercise is employed, it is customary to relate pressure readings in the leg to the simultaneously obtained blood pressure in the arm. A pressure gradient between the arm and the lower limb can be expressed in $\mathrm{mm} \mathrm{Hg}$ or as a ratio (ankle systolic pressure index).

Most studies report good correlation between the resting ankle pressure index and the degree of arterial narrowing as seen on the arteriogram, although a significant overlap exists between patients with peripheral vascular disease of increasing severity $(35,81,158,173)$. Pressure measurements at the level of the ankle, on the other hand, separate normal and diseased vascular systems with a high degree of accuracy, especially if the patient is exercised on the treadmill $(34,63,85$, $147,158,172)$.

If the ankle pressure indices at rest and after exercise are plotted against time, patterns become visible which are characteristic for the impairment of the circulation in a particular leg, and useful for objective evaluation of improvement or deterioration of an individual patient, since each leg serves as its own control (79). Several attempts to relate these pressure index patterns with commonly occurring localizations of atherosclerotic disease were unsuccessful $(36,79,87)$, emphasizing the general opinion that ankle systolic pressure measurements are useful in the functional assessment of arterial disease proximal to the ankle, while not providing information on its localization. 
In order to obtain such information segmental pressure measurements have been employed, although their practical value remains controversial as may be judged from the fairly high incidence of false negative or false positive findings in some studies concerning the diagnosis of arterial disease in the lower leg arteries and the aorto-iliac system $(3,31,35,40,60,85,148,150)$. Although mismatch between the occluding blood pressure meter cuff and the limb circumference may be recognized as à possible cause for inaccuracy $(85,158)$, an alternative explanation is proposed by Evans (52). A pressure cuff placed distal to a stenosis arrests flow completely if no major arterial branch leaves the main artery between the stenosis and the occluding cuff. As a result, pressure on both sides of the lesion becomes equal so that the pressure proximal to the stenosis will be determined when the cuff is slowly deflated. When a large arterial branch (e.g. the deep femoral artery) is present between the stenosis and the cuff a pressure gradient will be maintained, thus preventing falsely negative measurements.

McDonald (102) refers to Haldane's 'blinding glimpse of the obvious' with his statement that the oscillating pressure gradient along an artery is the driving force of pulsatile flow, thus stressing the importance of information possibly present in arterial flow and pressure wave forms when given as a function of time.

Plethysmographic mean volume flow and systolic pressure measurements show little resemblance to measurements of pulsatile hemodynamic phenomena. This may partly explain the noted deficiency of the pressure measurements to discriminate satisfactorily between arteriographic lesions of increasing severity. Johnston (81) was able to show that the pressure-time relationship of the systolic slope discriminated far better between atherosclerotic lesions of different severity than the ankle systolic pressure index.

Although the complete pressure-time wave form can be obtained through intra-arterial catheters at many sites in the human body without too great risks, one has to resign oneself to the fact that full information on the intra-arterial pressure cannot be determined noninvasively. This has stimulated the development of noninwasive techniques, primarily designed to obtain an alternative parameter as a substitute for pressure or volume flow.

\subsection{The velocity-time wave form and methods for its quantification}

Flow velocity has been recognized as an alternative parametcr of great practical value because it is intimately related to pressure and volume flow, but also because the velocity of blood can be determined noninvasively without difficulty (see section 2.7).

The oscillatory pressure gradient between any two given sites in the arterial system results in movement of blood (flow velocity), the amplitude and phase lag of its velocity in respect to the pressure gradient are determined by the heart rate, the radius of the artery and the inertia of blood (102). These three variables are also found in Womersley"s adaptation of Poiseuille's law for oscillatory flow in rigid tubes because volume flow in this case can be substituted by the mean vellocity across the vessel diameter.

The distensibility of arteries, which allows an artery to increase its diameter during systole with as much as $15 \%$ (48), is further complicating the already complex relationship between flow velocity and pressure, not only because the arterial diameter is constantly changing, but also, because the elastic properties of 
the vessel wall have great influence on the conduction of pressure and flow waves along the entire vascular system. The reflection of pressure waves from two functionally discrete sites in the upper and lower parts of the body has been recognized to be a major source for changes in the shape of the pressure and flow velocity wave forms $(102,117,159)$. In fact, these considerations were substantiated recently by Fourier analysis of the velocity wave form obtained from the femoral artery $(7,140,141)$. A third order Laplace transform was able to describe the velocity wave form of the femoral artery, its coefficients being related to the distal impedance, the proximall lumen diameter of the vessel and the elasticity of the vessel wall $(140,141)$.

These interesting studies corroborate the generally accepted view that the blood velocity-time wave form contains information relevant to the severity of an atherosclerotic lesion. The question remains how to extract this kind of information from the velocity wave form, without relying on high level technical sophistication, which at present is usually unavailable to the average clinician.

The methods employed in the past to quantify wave form changes have been taylored to the characteristic wave form shape of the particular artery under study. Carotid disease is manifested by the enhancement of the second peak during systole so that the ratio between the first and the second peak can be taken as a measure of disease (13). Changes in the systolic and diastolic amplitudes of the velocity wave form have also been used for quantification of carotid disease (106).

The velocity wave form of arteries in the lower extremity, on the other hand, has a reverse component which increases when going downstream $(69,102)$. The changes of the velocity wave form following arterial disease in this region are defined as damping and coincide with the disappearance of backflow and a decrease in amplitude and acceleration of the systolic peak. Although various different parameters, calculated from the outline of the wave form, have been described $(77,81,128)$, a dimensionless number representing the pulsatility of the wave form is thought to be the most useful parameter. The 'pulsatility index', of a wave form introduced by Gosling and co-workers (70), is defined as the ratio between the sum of the maximum oscillatory energy of the Fourier harmonics of the wave form and the energy of the mean forward flow. A good correlation was shown to exist between the pulsatility index (PI) thus defined, and a much simpler equation calculated directly from the outline of the velocity wave form $(67,80$,$) . This simplified version of \mathrm{PI}$, defined as peak-to-peak velocity divided by mean velocity during one cardiac cycle, made this parameter an attractive tool for the quantification of arterial disease. However, the results from several clinical studies revealed a significant overlap between femoral PI values in patients with different grades of aorto-iliac disease as judged from the arteriogram $(73,80,140$, 141). Although inaccuracy related to arteriographic reading may play a part ( 29 , 107), impedance of the terminal vascular system $(53,140,141)$, as well as the performance of the heart, may be important contributing factors to differences found between individuals (69).

In order to eliminate disturbing influences related to the condition of the vascular system proximal and distal to the arterial segment of interest, the degree of arterial damping over this segment was calculated by dividing the PI measured at the proximal site by the distally obtained PI $(80,170)$. Although reflections of the pressure wave at the site of stenosis or occlusion may induce changes in the proximal recorded velocity-time wave form and thus in the value of $P[(13,102$, 
112) this subject is not pursued at length by Gosling or Johnston. The effect of the peripheral vascular impedance on the Pl distal to a significant lesion $(53,140$, 141) has also remained undiscussed in relation to the clinical significance of damping. An additional limitation of this type of analysis is its difficult application in arteries not readily accessible to ultrasound, such as the aorta (69).

\subsection{The measurements of transit-time}

The time delay or transit-time (TT), between the arrival of the pulse wave at two distinctly separated arterial sites is determined by the pulse wave velocity and the length of the interposed arterial segment. Gosling (69) added this parameter of arterial function to PI measurements to improve their discriminative value for different degrees of arterial disease. TT is best measured from the foot of the wave form, whether it be the pressure, the pressure gradient or the flow velocity wave form, because early systole is least affected by reflected pressure waves and independent of the frequency composition of the wave form $(69,102)$.

The pulse wave velocity (PWV) along an artery is determined by its frequency composition, the viscosity of blood, the diameter of the artery but most of all by the compliance of the vessel wall $(102,116)$. Its relationship with vascular compliance is so strong that changes in the structural composition of the vessel wall accompanying aging and sexual maturation of young adults lead to measurable and reproducible differences of the PWV between individuals in a healthy young population (64). Because the intraluminal blood pressure also affects the compliance of the vessel wall $(27,69,71)$ it may be difficult to separate the influence of age and atherosclerosis on the PWV in patients with concomitant hypertension. Even greater difficulties will be met in the interpretation of the measured PWV in the presence of an arterial stenosis. The lower-than-normal poststenotic blood pressure and the atherosclerotic vessel wall changes have an opposite influence on the PWV, which might explain why no significant differences were found by Harris (73) between the PWV measured in normal and stenotic arterial segments. For this reason, a 'normalization' for mean blood pressure was proposed by Gosling (64) leading to a significant improvement of the discriminating qualities of TT foot-to-foot measurements. A recent study (77), employing identical methods, was not able to confirm these findings.

The effect of the vascular conduit length on TT, however, is of far greater magnitude, than the effect of blood pressure or age $(57,67)$ so that TT, if sufficiently prolonged, can be taken as a measure for the length of collateral pathways. In combination with the measurement of damping they have been used to describe the functional capacity of the collateral system $(57,67)$.

The combination of damping and TT, both derived from the velocity-time wave form, seems able to describe the function of the arterial segment between two measuring sites with a high degree of accuracy. When compared with arteriography an agreement level of over $90 \%$ was reported (69). The only type of instrument capable at present to provide the investigator with the means to study the velocity-time wave form noninvasively, is an ultrasonic velocity meter. 


\subsection{Doppler velocity meters: principle}

Characteristic for any Doppler instrument is the emission of ultrasound by a piezoelectrical crystal and the reception of back-scattered and reflected signals from moving targets and tissue interfaces, containing information relevant to their velocities. The received signal contains information on the velocity of moving erythrocytes within an artery or vein if the ultrasonic beam is directed at a blood vessel. The principle involved is simple and based on the Doppler effect, which can be expressed mathematically as follows:

$$
\Delta \mathrm{f}=\frac{2 \cdot \mathrm{f}_{\mathrm{e}} \cdot \mathrm{v} \cdot \cos \alpha}{\mathrm{c}}
$$

$\Delta f=$ frequency shift in backscattered signal

$\mathrm{f}_{\mathrm{c}}=$ emission frequency

$v=$ velocity of the moving ery throcyte

$\alpha=$ angle between ultrasonic beam and the direction of the moving erytrhocyte

$c=$ velocity of sound in tissue.

Assuming that $f_{e}, c$, and the angle $\alpha$ are constant, the detected frequency shift $(\Delta \mathrm{f})$ is directly proportional to the velocity of the ery throcyte. Doppler ultrasonic velocity meters, however, are less suitable to measure the absolute velocity $(\mathrm{m} / \mathrm{sec})$ of moving erythrocytes because the angle between the ultrasonic beam and the axis of the vessel is usually not precisely known under clinical conditions. Although several angle-independant Doppler systems have been described (e.g. 93) no clinical experience with this type of instrument has been reported.

\subsection{The arterial velocity profile and the Doppler frequency spectrum}

Poiseuille (1799-1869), according to McDonald, was the first to appreciate that individual erythrocytes under condition of steady flow had varying velocities across the diameter of cylindrical tubing owing to the viscous properties of blood. The ery throcy tes are flowing in a series of 'laminae' parallel to the wall of the tube and this flow is henceforth designated 'laminar flow'. The profile of the successive laminae across the tube under conditions of steady flow will be of parabolic shape. When flow is pulsatile, as in arteries, laminarity is maintained under normal conditions, although its velocity profile is seldom parabolic and more often of a 'plug' type $(5,54,102,124,125)$. These laminar velocity patterns are lost and turbulence arises when the Reynolds number, relating flow velocity to the diameter of the vessel as well as to the blood viscosity and density, exceeds a certain value as occurring for instance at the outlet of an arterial stenosis. This loss of laminarity has been observed on a lesser scale in the vicinity of vessel wall irregularities and in association with high flow velocities accompanying post-ischemic hyperemia (disturbed flow) (21).

The ultrasonic beam directed at the axis of an artery insonates its entire width, so that the back-scattered signal contains the velocity information of all fluid laminae and hence not a single frequency but a spectrum of frequencies. The bandwidth of this Doppler spectrum is relatively small owing to the 'plug type' velocity profile in the case of normal pulsatile flow. Frank turbulence is characterized by chaotic movement of erythrocytes with flow velocities in all directions 
causing spectacular broadening of the Doppler spectrum bandwidth with the simultaneous occurrence of positive (towards the probe) and negative (away from the probe) frequencies. It has been suggested that flow disturbances occurring at relatively slight degrees of arterial stenosis can be identified through the observed increase of the Doppler spectrum bandwidth $(21,124,134)$. However, the interpretation of slight spectral broadening is still under discussion since changes from 'plug' flow to more parabolic flow, while maintaining laminarity, will also be accompanied by spectral broadening (126).

\subsection{Continous Wave and Pulsed Doppler instruments}

The first clinical application of an ultrasonic velocity meter for the estimation of blood flow, described by Satomura (135) and Franklin (58), marked the beginning of a period in which stormy developments in Doppler technology took place. Ultrasonic methods in clinical medicine have greatly contributed to our knowledge of the physiology and pathophysiology of arterial blood flow, because the intravascular geometry and pattern of blood-movement remain undisturbed by ultrasound. Technological diversification, based on the principle of velocity detection with ultrasound, led to the development and construction of two essentially different instrumentations: continous wave (CW) and pulsed Doppler systems.

Any CW instrument uses a transducer containing two piezoelectrical crystals, one for continuous emission and one for subsequent reception of ultrasound. Directional Doppler instruments (103) soon replaced the non-directional earlier systems, because it became apparent that the direction of flow velocity was important in many clinical situations, as for example, when arteries and veins are closely aligned. At present a variety of this type of instruments is commercially available, with only marginal differences in their performance (139).

The transducer of a pulsed Doppler system usually holds one piezoelectrical crystal for the emission of short bursts of ultrasound, generated by gating the master oscillator. The same crystal is the receiver of back-scattered echoes in the comparatively long intervals between the emission of short ultrasound pulses, gated by means of an internal time-clock. The principal advantage of pulsed Doppler systems over $\mathrm{CW}$ Doppler instruments lies in their ability to measure the velocity of moving particles or structures at known distances from the transducer along the ultrasonic beam.

Pulsed Doppler systems, however, are of far greater technical complexity than $\mathrm{CW}$ instruments, because their mode of action is not only dependent on the chosen emission frequency, but also on the selected pulse repetition rate (PRF) and the duration of the emission. The PRF determines the maximum depth (R)* of velocity detection but also the maximum Doppler shift $(\Delta \mathrm{f})^{* * *}$ which can be detected accurately according to the Nyquist theorem $(9,10,75,76)$. Therefore, the measurement of high velocities at great depths is difficult. The depth of operation is also restricted by the duration of the ultrasonic pulse and the emission frequency because they determine the power of the back-scattered signal and thus the signal-to-noise ratio of the received signal.

The sampling properties of $\mathrm{CW}$ and pulsed Doppler systems are entirely different, due to the operational mode of each instrument. The output signal of a

* PRF $<c / 2 R_{\max }$.

* $\quad \Delta \mathrm{f}<\mathrm{PRF} / 2$. 
CW system contains velocity information from the entire insonated volume with decreasing contribution from moving bloodcells at increasing depths (tissue absorption) while a pulsed Doppler system obtains the velocity information from a small sample volume limited in the lateral direction by the ultrasonic beam width and in the axial direction by the sample gate width. The effective gate width depends on the duration of the emission, the band width of both the transducer and the input section of the instrument, as well as on the low pass filter in the phase detector $(75,76)$. Any pulsed Doppler system should be tailored to its intended application, as illustrated by differences between the technical specifications of several experimental and clinical reports $(5,8,54,154)$ calling for elaborate compromises in the choice of the emission frequency, the PRF and the duration of the emission $(10,75,76)$.

\subsection{Single-and multi-gate pulsed Doppler systems}

Single-gate pulsed Doppler systems have been used to study the velocity of an artery at known distances to the transducer, undisturbed by vessel wall movements and venous flow $(5,54,118)$. They allow the determination of the instantaneous mean velocity at only one site in the vessel so that many consecutive heartbeats are required for synthesis of the velocity profile. In addition to the poor reproducibility of the velocity profile in the vicinity of an arterial stenosis cluring subsequent heartbeats, problems are encountered in positioning and maintenance of the sample volume along the range of interest. Their combined use with B-mode echo $(9,20,21)$ facilitates this task but considerably reduces the maximum detectable frequency in the artery.

In order to eliminate some of these limitations, multi-gate pulsed Doppler instruments were developed $(25,26,75,76)$ with the ability to detect, simultaneously and instantaneously, velocities over the full range of interest. The instantaneously determined velocity profile allows better control of the measurements because time-related changes in the sagittal and lateral angles of the insonating beam are minimized. Bench-model measurements with multi-gate Doppler instruments $(25,26,49)$ show surprisingly good agreement with the test tube diameter although the finite dimensions of the sample volume (sample resolution) are still considered a limiting factor. In the latest multigate pulsed Doppler system, developed by Hoeks $(125,126)$, velocity samples can be taken along the ultrasonic beam at distances of $0.5 \mathrm{~mm}$ (sample volume about $1 \mathrm{~mm}^{3}$ ) and as close to the vessel wall as $0.5 \mathrm{~mm}$.

\subsection{Methods of signal processing}

The ways in which output signals from Doppler instruments can be processed are numerous and should be adjusted to the Doppler instrumentation or to the parameter of intended study derived from the Doppler signal. The demodulated output of any Doppler instrument can be presented to the examiner as an audiosignal for immediate interpretation, because the human ear is able to recognize characteristic frequency patterns within the audiosignal as associated for example with normal flow or turbulence. Classification of audiosignals is the simplest and most widely practised form of 'signal-processing' among clinicians, which estab- 
lished Doppler velocity meters in clinical practice $(3,31,85,157,172)$. Its subjective nature prevents scientific comparison, so that methods were developed and tested to quantify the Doppler signal. Quantification of the Doppler signal can be performed by electronic averaging of the Doppler frequency spectrum to its instantaneous mean frequency, or by display of the Doppler frequency spectrum itself, both of which can be done in various ways.

A zero-crossing meter, commonly applied in commercially available Doppler instruments, determines the mean frequency of the Doppler spectrum in analog form. However, it is only under ideal conditions that the output is linear to the instantaneous average frequency in the Doppler signal, and thus to the instantaneous average velocity in the insonated vessel. Low signal-to-noise ratio's $(6,94$, $123,127)$, contamination with venous signals $(67,80)$ and vessel wall movements $(123,124)$, may introduce serious error in the conversion of the Doppler signal into a voltage assumed to be proportional with the average frequency in the Doppler frequency spectrum. The analog output signal of zero-crossing meters may also suffer serious distortion from surrounding electrical equipment and from shortwave radio transmissions (67). Much attention has been paid to the error in the determination of the average velocity resulting from an increased band width of the Doppler frequency spectrum. This fundamental problem of the zero-crossing meter accounts for an estimated error of $16 \%$ to $20 \%(94,129)$ whereas all above factors together, may be responsible for error varying from twenty to several hundred percent $(80)$.

An entirely different method for the determination of the average velocity of the Doppler signal was proposed by Arts (6) and Reid (122) utilizing the power density spectrum of the receiver signal. The limitations inherent in the use of a zero-crossing meter are hereby eliminated. However, venous signals and artefacts remain potential sources for error.

Zero-crossing meters possess great merit owing to their ability to condense all the information present in the Doppler signal into a single line but it can be argued with equal justification that much information about the pattern of flow is hereby lost (126). If spectral anallysis of the Doppler signal is employed, sonagrams are produced in which the various frequencies in the signal are given as an instantaneous function of time, while their amplitude is represented by the intensity of the sonagram pattern. Proponents of the use of spectral analysis $(20,21,57,69,80,170)$ are of the opinion that none of the above mentioned limitations and disadvantages of the zero-crossing meter are applicable to this method, because arterial signals, displayed as a clearly defined and relatively narrow frequency band, are easily distinguished from venous signals, artefacts and noise. This arterial high frequency envelope broadens and assumes an irregular outline in the presence of disturbed flow or turbulence due to associated random changes in the flow velocities that occur at any time during the cardiac cycle. These changes can be of diagnostic value $(20,21,54,124,134)$ for relatively slight degrees of stenosis.

\subsection{Pulsatility index, transit-time and the Doppler velocity meter}

The calculation of PI and the measurement of TT (sections 2.5 and 2.6) can be performed either on analog velocity signals or on sonagrams obtained with $\mathrm{CW}$ or pulsed Doppler instruments. PI is calculated from the outline of the "high fre- 
quency envelope' in the sonagram or directly from the analog mean velocity wave form. When normal flow patterns (plug-type) in the artery are present, the contours of the 'peak' and 'mean' velocity wave forms, and hence the calculated PI, may be identical. The analog wave form however is sensitive to distortion from many causes beyond the examiner's control, for which reason spectral analysis is preferred by most workers in this field $(57,69,80)$.

The Doppler velocity wave form, whether presented as a sonagram or as an analog signal, is suitable for the calculation of PI, because this dimensionless number is virtually independent of the angle between the ultrasonic beam and the direction of blood velocity. According to the definition of PI, both numerator and denominator are sensitive to the angle of insonation. The high degree of accuracy of damping and TT measurements as reported by Gosling (69) has not been equalled by others, presumably because two Doppler instruments were simultaneously used to obtain the PI proximal and distal to an atherosclerotic lesion. It is stated $(57,69)$ that the simultaneous recording of the sonagram at two sites along the arterial pathway prevents unwanted influences from time-related changes of the circulation (cardiac arrhythmia's, respiration) on the shape of the wave form and hence on PI and TT. However, the significance of these time-related changes on the accuracy of the employed method has never been convincingly established. Angelides (4) was able to differentiate between occlusions at different levels of the arterial pathway using only one commercially available CW Doppler instrument!

It was stated in the beginning of this chapter that a noninvasive method, besides its scientific merits, must be cheap and easy to operate, so that a busy physician can carry out the necessary diagnostic work-up in a practical manner. The recording of two simultaneous signals requires two technicians to hold the transducers, which is neither easy nor cheap. Another disadvantage of the method proposed by Gosling (69) concerns the off-line calculations of Pl, damping, and TT from the sonagrams, which is certainly time-consuming. It is not impossible that this aspect of the otherwise elegant method, proposed by the Guy's group, has been a limiting factor to its widespread use in clinical circumstances.

\subsection{Aim of the present study}

Noninvasive diagnostic methods, employed to detect and quantify the extent of disease along the arterial segments between two sites of measuring, are in great demand by clinicians who face difficult decisions regarding the 'if" and 'where' of an arterial reconstruction especially in the presence of multi-level disease.

The previous section discussed the time-consuming and laborious nature of the method first proposed by Gosling. Obviously, on-line measurements of PI and TT using one transducer, are to be preferred if it can be shown that acceptable accuracy is maintained. Averaging of PI and TT over a large number of heart beats may be carried out to eliminate the influence of some time-related phenomena such as cardiac arrhythmia's. The R-wave of the ECG may be used as a fixed reference for the detemination of the TT. On-line TT measurements are fairly easy to achieve electronically, using the systolic peak of an available singlevoltage signal which rules out the use of spectral analysis.

The limitations and disadvantages of zero-crossing meters (section 2.11) which provide the analog velocity signal for on-line signal processing, may not be appli- 
cable when used in pulsed Doppler instruments (94). The frequency bandwidth of the Doppler signal obtained from a midstream sample volume is small, whereas yenous and vessel wall signals are left aside.

Modern multi-gate pulsed Doppler systems (section 2.10) have greatly facilitated the examiner's task to achieve controlled sampling in the artery of interest along the ultrasonic beam, meanwhile supplying relevant information about the diameter of the insonated vessel.

It is therefore proposed to study the accuracy of on-line PI and TT measurements, averaged over a number of heart cycles, for the functional description of a vascular segment. Only one transucer is used at a time, in combination with a commercially available CW Doppler instrument (Versatone D9-Medsonics) and the multi-gate pulsed Doppler system as built in Maastricht by Hoeks (76). The noninvasive parameters are computed with a custom-built wave form analyser (138) from the analog velocity wave form provided by both instrumentations. The noninvasive parameters will be compared independently with the arteriographic information.

The femoro-popliteal area gives ample opportunity for the evaluation of this technique, because the common femoral artery and the popliteal artery are both easily accessible to ultrasound. Local disease in the common femoral and the lower popliteal arteries is relatively uncommon if compared to the prevalence of obstructive disease in the superficial femoral artery. Error in measurement, caused by clisruption of normal flow patterns resulting from disease at the site of measuring, may therefore be small. An extensive description of the materials and methods will be given in the next chapter. 


\section{Materials and methods}

\subsection{Introduction}

This study deals with a noninvasive method for the functional description of atherosclerotic lesions in the femoro-popliteal area. Detailed information about the patients' signs and symptoms is omitted, because they refer to the general vascular status and lifestyle of the patient rather than provide objective information on the extent of disease in the limited area of the femoro-popliteal pathway.

\subsection{The patients}

One hundred and seven new patients with clinical signs or symptoms of vascular disease, referred to the vascular laboratory of St.Annadal Hospital between 1.5.1979 and 1.5.1980, were studied according to the protocol outlined in section 3.5. of this chapter. This series is not consecutive, mostly because patients were asked to participate only if future arteriography was anticipated prior to further treatment. An additional group of 41 patients without clinical signs or symptoms of vascular disease, usually referred to the vascular laboratory to exclude the presence of significant vascular disease, were also admitted into the study primarily to serve as a control group. The distribution of sex and age over both groups (table 3.1) shows the expected preponderance of males in all age groups with the highest incidence of vascular disease in the six th decade.

Table 3.1 Specifying age and sex for all 296 legs included in the study. ( ) are legs from the control group.

\begin{tabular}{lccccccc}
\hline Age & $30-39$ & $40-49$ & $50-59$ & $60-69$ & $70-79$ & $80-89$ & Total \\
\hline Sex & & & & & & & \\
Male & $2(8)$ & $10(24)$ & $46(8)$ & $56(10)$ & $46(8)$ & $6(0)$ & $166(58)$ \\
Female & $2(2)$ & $4(4)$ & $18(4)$ & $8(8)$ & $6(4)$ & $10(2)$ & $48(24)$ \\
\hline Total & & & & & & & $296(82)$ \\
\hline
\end{tabular}

A leg was considered to be normal and free from significant arterial disease if the ankle systolic pressure index (ASPI) at rest and after exercise was equal to or more than $95 \%$ (sections 2.4 and 3.5 ). Thus, all legs with pressure indices below this value were considered pathological.

Not all patients with symptomatic arterial disease were affected bilaterally and some of the 82 legs belonging to the group of 41 patients without clinical 
signs or symptoms of vascular disease were shown to have a pathological ASPI at rest or after exercise. The ASPI 's were incomplete in 37 legs for a variety of reasons, such as dyspnoea, angina, restpain in a leg or incompressible arteries at the level of the ankle. Ninety-five legs were considered normal according to the criteria outlined above, so that 164 legs with significant arterial disease (table 3.2.) and completed ASPI's could be investigated.

Table 3.2 The distribution of normal, pathological and incomplete ankle systolic pressure indices (ASPI) over 188 legs in which arteriographic visualization was carried out. TLA = translumbar aorto-arteriography; punc. fem. = direct puncture of the femoral artery.

\begin{tabular}{lcccc}
\hline & $\begin{array}{c}\text { Normal } \\
\text { ASPI } \\
\geqslant 95 \%\end{array}$ & $\begin{array}{c}\text { Pathological } \\
\text { ASPI } \\
<95 \%\end{array}$ & $\begin{array}{c}\text { Incomplete } \\
\text { ASPI }\end{array}$ & No. of legs \\
\hline Translumbar aorto-arteriography & 14 & 94 & 12 & 120 \\
TLA + punc. fem. & 2 & 12 & 6 & 20 \\
Punc. fem. & 5 & 16 & 15 & 36 \\
Seldinger aorto-arteriography & 3 & 9 & 0 & 12 \\
No arteriographic visualization & 71 & 33 & 4 & 108 \\
\hline Total no. of legs & 95 & 164 & 37 & 296 \\
\hline
\end{tabular}

\subsection{Arteriography}

In 188 of the 296 legs arteriographic visualization was performed by means of translumbar aorto-arteriography, direct puncture of the femoral artery, arteriography according to the Seldinger technique or by a combination of these procedures (table 3.2.).

Each anatomically defined arterial segment (table 3.3), from the aorta down to the lower leg arteries, was classified as normal, stenotic or occluded depending on its arteriographic appearance. The peroneal artery was exempted from systematic reading and classification because it was usually poorly visualized on the arteriogram. A micrometer was used to measure the diameter of the narrowest part of each vascular segment. Arterial segments were often inadequately visualized or not visualized at all in a particular arteriogram, so that reliable judgment in those cases was not possible. These segments were graded separately, together with the corresponding arterial segments of 108 legs in which no arteriography was carried out (table 3.3.). Some explanatory comments are indicated here to justify this seemingly gross and subjective classification of the arteriographically visualized lesions, because they are of crucial importance for the interpretation of the results of this study.

It became clear during the preparatory phase of the current study that singleplane arteriograms could be misleading as to the true extent of the degree of arterial. narrowing present in an arterial segment. The results of a separate study concerning the interobserver agreement on arterial lesions as observed on singleplane aortographies revealed a low degree of interobserver agreement on stenotic lesions if they were classified as stenoses with more than $50 \%$ or less than $50 \%$ 
Table 3.3 Thirten arterial segments were defined in 188 arteriograms (table 3.2 ) and classified as normal, stenosis or occlusion. Depending on the type and/or quality of these arteriograms, classification was not possible in a substantial number of these legs. They were added to 108 arterial segments from legs in which no arteriographic visualization was carried out (missing values).

\begin{tabular}{lcccc}
\hline & normal & stenosis & occlusion & missing yalues \\
\hline Aorta & 170 & 4 & 0 & 122 \\
Common iliac artery & 136 & 26 & 8 & 126 \\
External iliac artery & 149 & 15 & 6 & 126 \\
Common femoral artery (CFA) & 160 & 4 & 3 & 129 \\
Bifurcation of the CFA & 137 & 22 & 2 & 135 \\
Deep femoral artery & 144 & 21 & 3 & 128 \\
Superficial femoral artery (proximal) & 98 & 28 & 44 & 126 \\
Superficial femoral artery (distal) & 63 & 36 & 71 & 126 \\
Popliteal artery (above kneejoint) & 138 & 17 & 12 & 129 \\
Popliteal artery (below kneejoint) & 152 & 7 & 6 & 131 \\
Trifurcation & 126 & 10 & 6 & 154 \\
Posterior tibial artery & 116 & 5 & 13 & 162 \\
Anterior tibial artery & 116 & 8 & 9 & 163 \\
\hline
\end{tabular}

obliteration of the arterial lumen (29). These findings confirmed the observations of other investigators in similar studies $(46,62,176)$, that considerable error may be expected in the estimation of the degree of narrowing if this classification is applied.

It was therefore decided to classify stenotic lesions according to their presumed functional significance, thereby relying entirely on the examiner's experience. The examiner was thus allowed to interpret caliber changes of the arterial lumen, subtle indentations on the outline of the vessel as well as changes of the intraarterial contrast density, while comparing the area of maximal narrowing with the diameter of the normal arterial segments in the same arteriogram. The micrometer measurements were not considered useful in this grading process because they disregard generalized disease and supply information about the vessel diameter in only one direction.

It is realized that arterial grading of this kind is highly subjective and open to error; however, no solution to this problem seems available at present. Verifying intra-arterial pressure measurements in the femoral artery were not routinely carried out and segmental noninvasive pressure measurements, especially in the upper thigh region are known to lack surficient accuracy (section 2.4).

An arterial segment was thus graded 'stenotic' if a pressure drop was assumed to exist over the area of observed arterial narrowing whether at rest or after exercise. A narrowed arterial segment not thought to be associated with a pressure drop was classified as normal. Arterial segments displaying a totally obliterated lumen were classified as 'occlusion' irrespective of the length of the occlusion. No attempts were made to classify collaterals.

The difficulties encountered in arteriographic interpretation and the reliability of ankle systolic pressure measurements for signalling significant atherosclerotic disease (section 2.3) above the level of the lower leg arteries, were the reason that 
pressure measurements could overrule observations from arteriography. If pressure indices before and after exercise were normal $(\geqslant 0.95)$, all arterial segments were considered to be normal. The lower leg arteries are excluded from further analysis, because they are expected to have little effect on the results of the present study. The distal part of the popliteal artery is also excluded, because the ultrasonic measurements in the knee were carried out somewhere at the level of the joint space, well above the distal part of the popliteal artery. Lesions in this particular artery are comparatively rare (table 3.3 ), so that the possible influence of reflected pressure waves on the velocity signals obtained in the proximal part of the popliteal artery would affect the results of this study only to a limited extent.

$\begin{array}{ll}0 & \text { Normal } \\ 1 & \text { SAI } \\ 2 & \text { SAl SSFA } \\ 3 & \text { SAl SSFA Spop } \\ 4 & \text { SAl Spop } \\ 5 & \text { SAl Opop } \\ 6 & \text { SAl Sprof } \\ 7 & \text { SAl S SFA Sprof } \\ 8 & \text { SAI OSFA } \\ 9 & \text { SAI OSFA Sprof }\end{array}$

ASPl at rest
10 SAI OSFA O pop

11 SAI OSFA S prof Spop

12 OAl

13 OAI SSFA S pop

14 OAI OSFA Opop

15 SSFA

16 SSFA S prof

17 SSFA S pop

18 SSFA Opop

19 SSFA Opop Sprof
20 SSFA Oprof Opop 21 OSFA

22 OSFA Spop

23 OSFA Oprof

24 OSFA O pop

25 OSFA Sprof

26 OSFA S prof O pop

27 OSFA Sprof Spop

28 OSFA S prof O pop

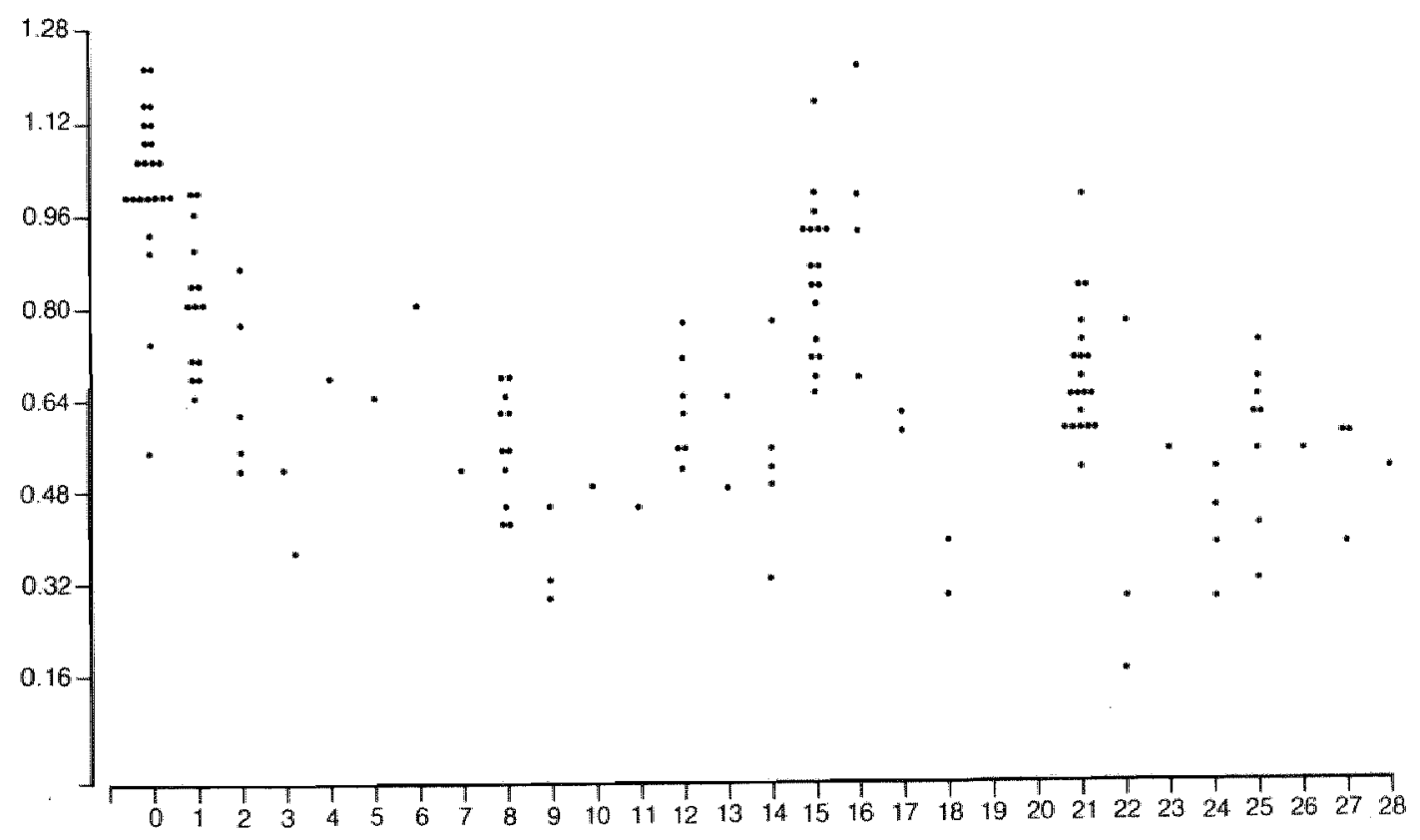

Fig. 3.1 Complete arteriographic information (from aorta to the popliteal artery) in 143 legs is set against the ankle systolic pressure index (ASPI) at rest. Twenty eight different combination $(0-28)$ of disease $(\mathrm{S}=$ stenotic, $0=$ occlusion) in the aorto-iliac-common femoral segment (AI), the superficial femoral artery (SFA), the deep femoral (prof) and the popliteal artery (pop) were made. Four legs with normal arterial systems from the aorta down to the popliteal artery had significant arterial lesions in the lower leg arteries explaining their ASPI less than $95 \%$. 
Three vascular areas are of interest. The aorto-iliac-femoral (AI) segment provides the arterial inflow of the area under study, covering the vascular system from the aorta down to the bifurcation of the common femoral artery. The superficial femoral artery (SFA) and the proximal part of the popliteal artery compose the arterial segment used for comparison with the noninvasive data. The deep femoral artery and its origin in the common femoral artery are separately classified, being part of the main collateral pathway in this area.

Complete arteriographic information from the aorta down to the popliteal artery was available in only 143 legs. The non-homogenous character of the group of patients with symptomatic arterial disease is well illustrated in figure 3.1.

\subsection{The instrumentation}

Detailed technical descriptions and block-diagrams concerning the multi-gate pulsed Doppler system (76) and the continuous wave (CW) commercial Doppler vellocity meter (Versatone D9-Medsonics) are available elsewhere. Only a short summary will be given here (table 3.4 ).

Table 3.4 The technical characteristics of the multi-gate pulsed Doppler system.

emission frequency
emission duration
pulse repetition frequency
maximum velocity detection
in direction of ultrasonic beam.
beamwidth
axial sample resolution
cut-off frequency high pass filter
cut-off zero-crossing filter

$6 \mathrm{MHz}$

1 or $2 \mu \mathrm{sec}$

5,10 or $20 \mathrm{kHz}$

$1.2 \mathrm{~m} / \mathrm{sec}$ at $20 \mathrm{kHz}$

in direction of ultrasonic beam.

beamwidth

axial sample resolution

cut-off zero-crossing filter

$1 \mathrm{~mm}(-6 \mathrm{~dB})$ from $10-40 \mathrm{~mm}$ along the beam

$1.2 \mathrm{~mm}$

$300 \mathrm{~Hz}$

$7.5 \mathrm{~Hz}$ (first order low pas filtering)

The basic configuration of the multi-gate pulsed Doppler system (fig. 3.2) consists of an enitter, a phase detector, an audio filter and a zero-crossing detector followed by a low-pass filter. One $5 \mathrm{~mm}$ piezoelectrical crystal is mounted in a pencil probe and employed alternately as emitter and receiver. Short emissions of ultrasound (emission frequency $6 \mathrm{MHz}$, duration 1 or $2 \mu \mathrm{sec}$ ) are constantly repeated at preselected pulse repetition frequencies (PRF) of 5,10 or $20 \mathrm{kHz}$. An axial sample distance of $1 \mathrm{~mm}$ was selected, so that 32 adjacent sample volumes restrict the maximum $\mathrm{PRF}$ to $20 \mathrm{kHz}$, allowing the detection of velocities relative to the transducer up to $1.2 \mathrm{~m} / \mathrm{sec}$ over the depth range of $32 \mathrm{~mm}$. Examinations on patients with a PRF of $20 \mathrm{kHz}$, however, could not be maintained for a long period of time owing to the development of heat at the crystal interface. The emission duration of $1 \mu \mathrm{sec}$ provides the best resolution along the ultrasonic beam and matches the selected sample distance of $1 \mathrm{~mm}$. The range of interest in the femoro-popliteal region was usually between 10 and $20 \mathrm{~mm}$, so that the signalto-noise ratio was adequate for normal measuring conditions.

The lateral dimension of the sample volume depends on the local beamwidth. A transducing crystal of $5 \mathrm{~mm}$ in diameter, mounted behind a perspex lens, 


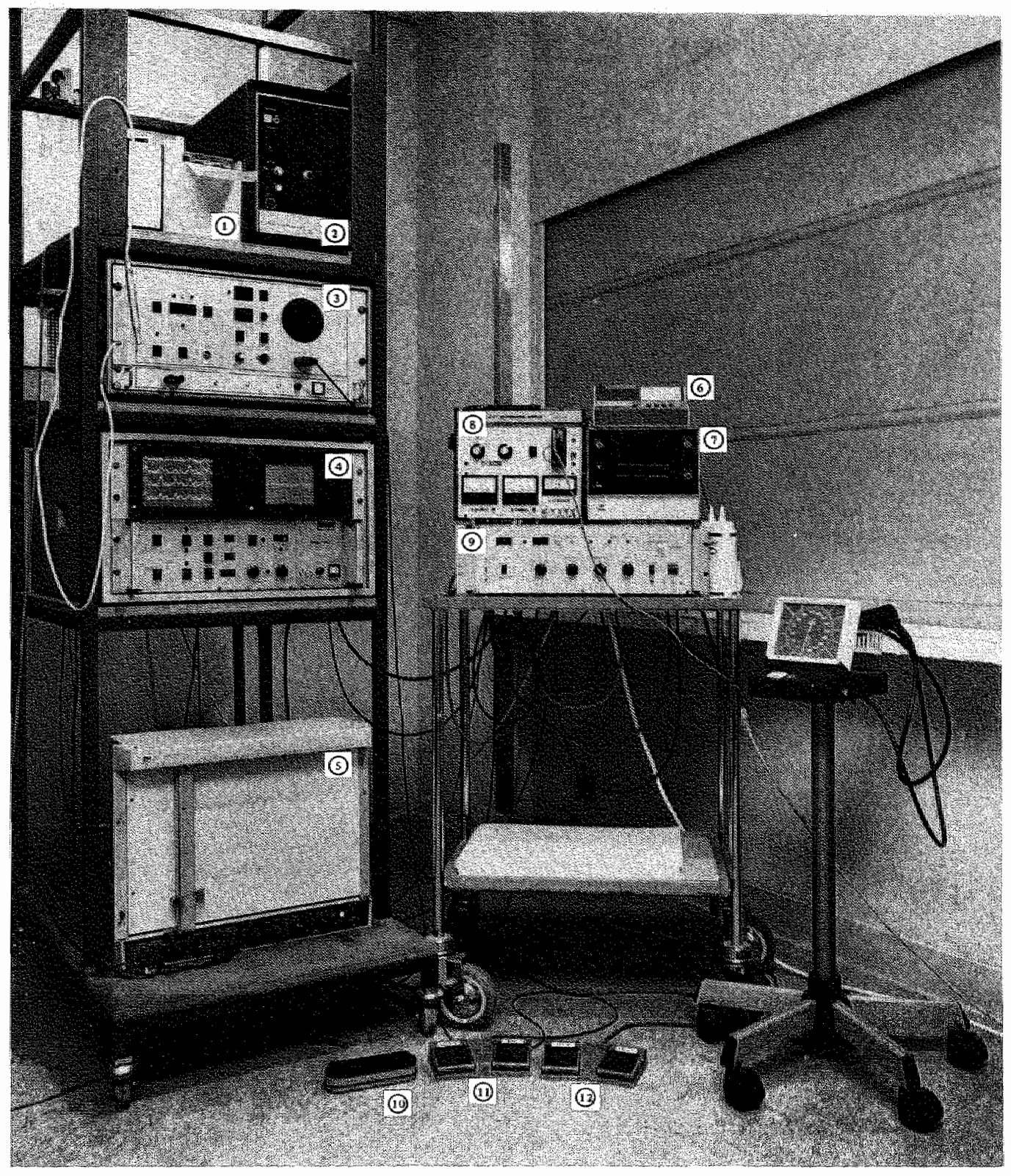

Fig. 3.2 1 threechannel physiological recorder (Oscillomink-Siemens)

2 E.C.G. trigger

3 multi-gate pulsed Doppler system

4 multi-gate storage display unit

$5 \quad X$ Y plotter (Hewlett-Packard)

6 printer: Printina-SR alpha-numerique

7 oscilloscoop for Versatone signals

8 Versatone D9-Medsonies

9 digital wave form analyser

10 footswitch for three channel physiological recorder

11 footswitch to select sample volume

12 footswitch to initiate $\mathrm{PI}$ and DT recording with printer 
focussed at $25 \mathrm{~mm}$ which results in a beamwidth of less than $2 \mathrm{~mm}(-6 \mathrm{~dB}$ level) from 10 to $40 \mathrm{~mm}$ along the beam.

A "phase sample" represents the average phase difference, averaged over the sample volume, between the received signal and the reference signal (emitted signall), weighted by the amplitude of the received signal. Successive phase samples originating from a single sample volume produce the familiar Doppler signal sampled by the PRF. With multi-gate pulsed Doppler systems phase sampling with a rate of $750 \mathrm{kHz}$ is employed to assure an axial resolution of $1 . \mathrm{mm}$. Only one signal processing circuit is employed in serial data processing, so that phase samples, taken from a number of adjacent sample volumes, are processed via a single circuit (high-pass filtering followed by zero-crossing counting). Information on the flow direction is preserved by quadrature phase detection.

During each processing cycle the instantaneous mean velocity in each of the adjacent sample volumes appears in serial form at the velocity profile output. Processing is done on a digital base so that a digital-to-analog converter had to be inserted for visual display.

The Doppler audio signal in each of the 32 gates could be selected by a footswitch, its position in relation to the other gates clearly indicated on the visual display as a bright dot (fig. 3.2). The zero-crossed analog signal of this gate was simultaneously available for further analysis (fig. 3.3). In order to retain velocity data of all sample volumes, a special multi-channel storage display was developed,
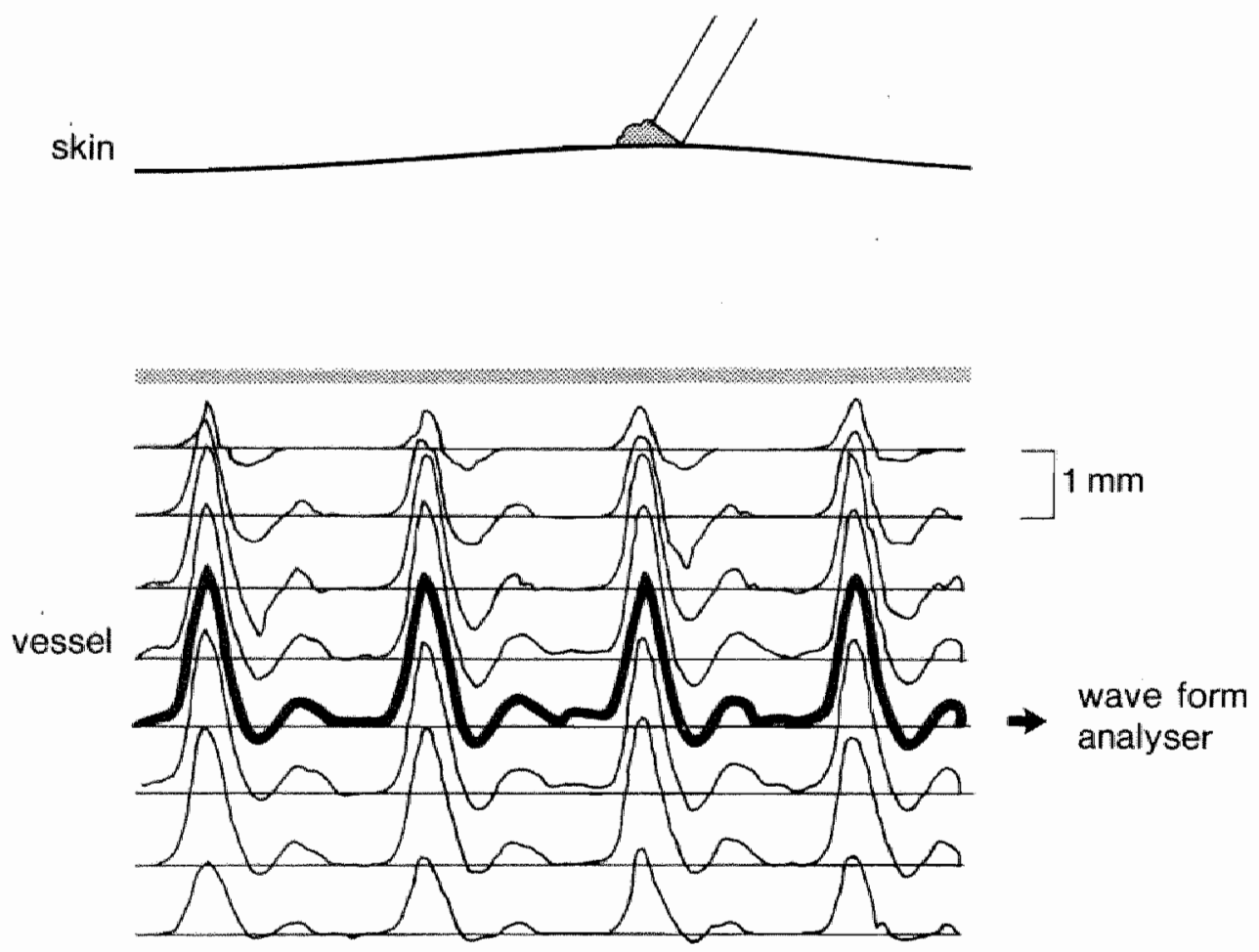

Fig. 3.3 Schematic presentation of a centerstream velocity measurement with the pulsed Doppler system. 
holding the last recorded velocity profiles up to a maximum of 256 profiles.

Two modes of display are available. In the depth mode (fig. 4.3) the velocities are presented as a function of depth at discrete time intervals during the cardiac cycle, while the time mode (fig. 3.7, 3.8, 3.10,3.11 A) shows the velocities as an instantaneous function of time in each individual gate. The instrument was usually operated in the time mode because the main interest was in the temporal. behaviour of velocities. An off-line X-Y plotter (Hewlett-Packard provided hard copies.

The CW Doppler instrument used was the commercially available Versatone D9-Medsonics (fig. 3.2). No technical modifications were made. The zero-crosser output was connected to a three-channel physiological recorder (OscillominkSiemens) operated by a footswitch, providing hard copies of the analog velocitytime wave form (fig. 3.13). The main characteristics of the instrument, as specified by the manufacturer, are an emission frequency of $8 \mathrm{MHz}$ and a band pass filter with a range of 0.1 to $12 \mathrm{kHz}$.

A digital waveform analyser (fig. 3.2) was especially developed for on-line computation of pulsatility index (PI) and delay-time (DT) from the analog velocitytime signal as obtained from the output of both Doppler instrumentations (138) ${ }^{\text {*k }}$. The essential components of the instrument are an analog-to-digital converter with a conversion rate of $400 \mathrm{~Hz}$ and an 8 bits microprocessor ( $8085 \mathrm{~A}-2$, microprocessor from Intel).

Maximum $\left(\mathrm{V}_{\max }\right)$ and minimum $\left(\mathrm{V}_{\min }\right)$ values of $\mathrm{V}_{(\mathrm{t})}$ as well as the timeaveraged velocity $\bar{V}_{(t)}$ are respectively determined by peak detection and integration using the microprocessor. $V_{\text {peak-to-peak }}$ and PI are subsequently calculated

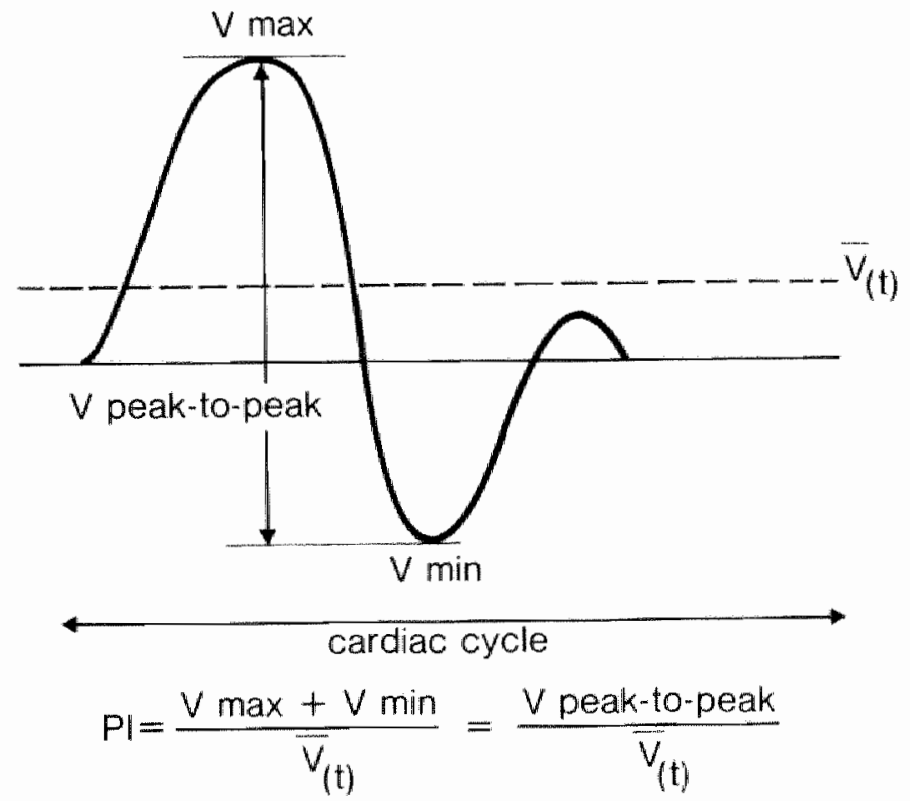

Fig. 3.4 $V_{\max }, V_{\min }$ and $\bar{V}_{(t)}$ are on-line determined by the wave form analyser for on-line calculation of $\mathrm{V}_{\text {peak-to-peak }}$ and PI.

* Detailed technical information is available on request with the author. 


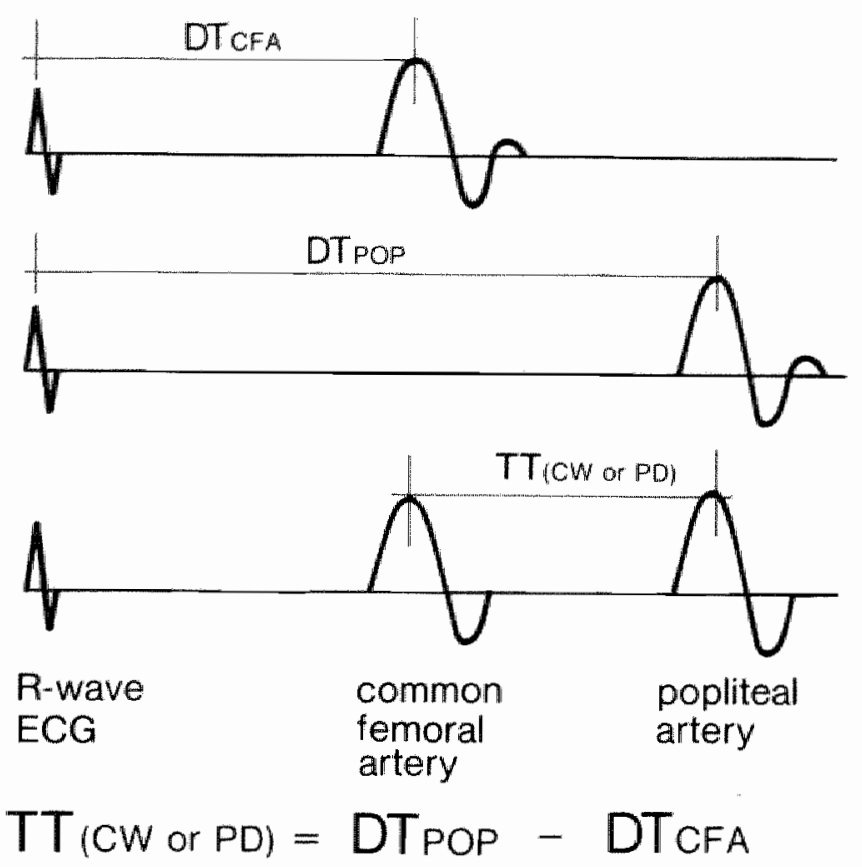

Fig. 3.5 The computation of $\mathrm{TT}_{(\mathrm{CW}}$ or PD) is schematically shown. $\mathrm{DT}_{\mathrm{CF}}=$ delay-time between the heart and the systolic velocity peak at the common femoral artery; DT $_{\text {pop }}=$ delay-time between the heart and the popliteal artery.

(fig. 3.4). The peak of the R-wave of the ECG is used to initiate the computation, but it served also as a point of reference for the measurement of the DT.

The delay-time between the R-wave of the $\mathbb{E C G}$ and the arrival of the systolic velocity peak at a proximal (DT $\mathrm{CFA}_{\mathrm{A}}$ ) and distal $\left(\mathrm{DT}_{\text {pop }}\right.$ ) site of the femoropopliteal pathway is determined, so that TT can be calculated by subtraction of both delay-times (fig. 3.5).

Fig. 3.6 illustrates the difference between TT peak-tomeak as compared to TT measured at the foot of the velocity wave form. The presence of an arterial occlusion forces the velocity wave through a network of collaterals which has a

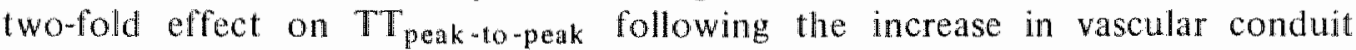
length and the occurrence of damping. The increase in vascular conduit length is best measured at the foot of the wave form ( $T T_{\text {foot-to-foot }}=b$, fig. 3.6) while further temporal displacement of the systolic peak, caused by the sluggish upstroke of the velocity wave (c), can be quantified by measurements on the peak of the wave form. TT peak - to-peak may therefore be a stronger parameter for athero-

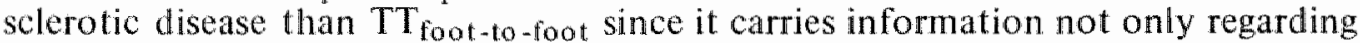
the length of the vascular pathway but also on the severity of the arterial lesion.

Although the wave form analyser was able to compute PI and $\mathrm{DT}_{\text {peak-to-peak }}$ for each individual heartcycle, an averaging modality was provided to calculate mean parameter values over a preselected number of heartbeats. The values of PI and DT were averaged over four heartcycles and displayed on the front panel of the instrument, thereby greatly facilitating the examiner's task to reject parameter values well outside the range. Sampling of 4 -heartbeats averaged values was contimued over altogether 20-30 heartbeats. A series of 5-8 4-heartbeats averaged 


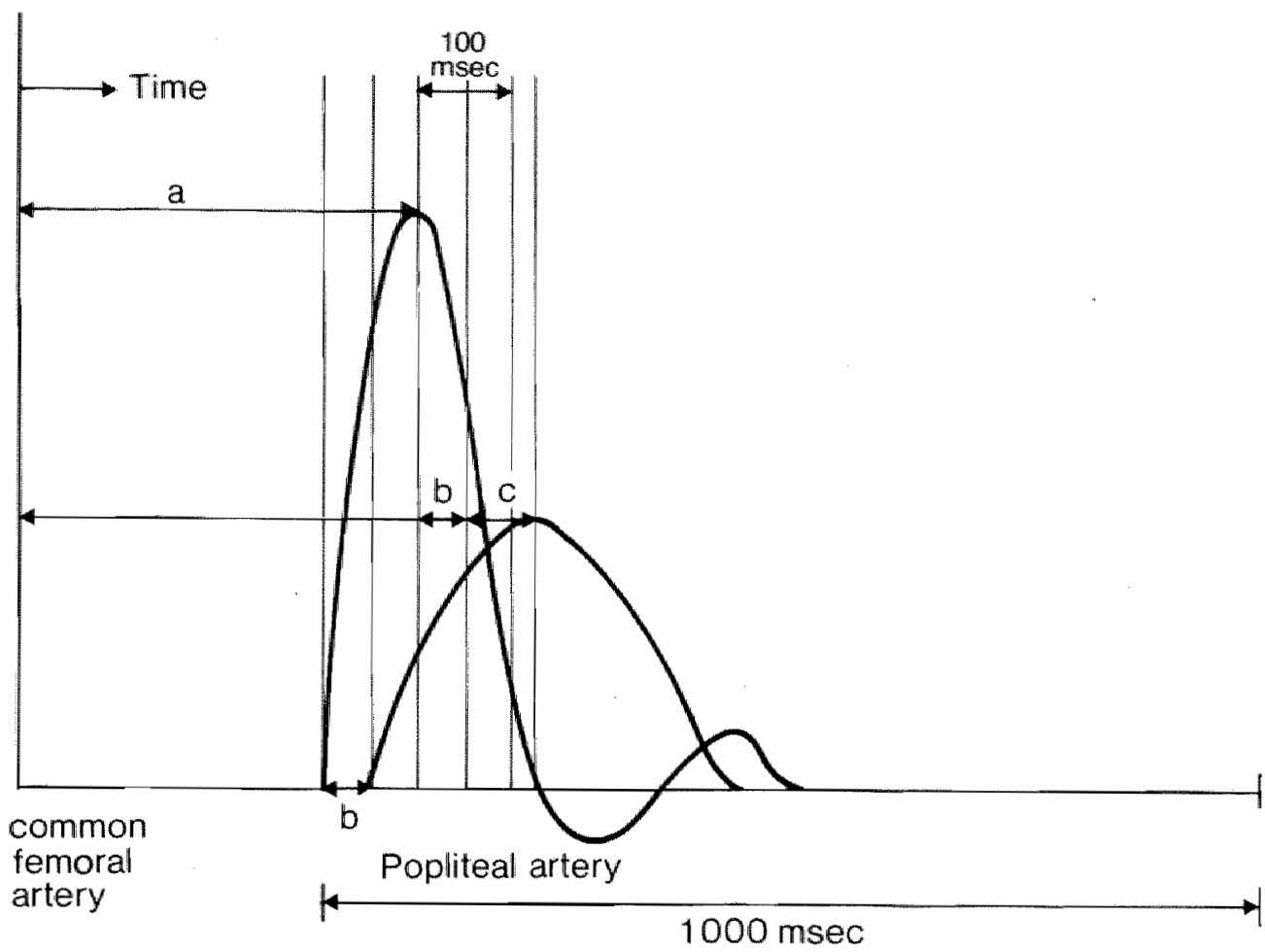

Fig. 3.6 Schematic diagram to show the difference between $\mathrm{TT}_{\text {foot-to-foot }}$ and $\mathrm{TT}_{\text {peak-to peak }}$ $\mathrm{a}=$ transit-time common femoral artery - normal popliteal artery (peak-to-peak),

$\mathrm{b}=$ increase of transit-time caused by longer arterial pathway in case of occlusion of the SFA (foot-to-foot).

$c=$ additional increase of transit-time caused by damping (peak-to-peak).

$a+b+c=$ transit-time between the common femoral artery and the popliteal artery in case of occlusion of the SFA (peak-to-peak).

values of PI, DT, $V_{\max }, V_{\min }$ and $\bar{V}_{(t)}$ were printed by the Printina-SR alphanumerique, operated by a footswitch.

\subsection{Examination of the patient}

The time necessary to complete an examination was usually $1 \frac{1 / 2}{2}$ to 2 hours, individual differences being dependent on the quality of the Doppler signals and the cooperation of the patient. Poor signal-to-noise ratio's were usually associated with extensive atherosclerotic arterial lesions which made it difficult to obtain optimal signals, thus prolonging the examination-time.

The patients were asked to rest on a couch for 20 minutes. Three precordial ECG electrodes were applied to trigger (fig. 3.2) the velocity wave form analyser and a skin marker was used to outline the inguinal ligament, connecting the anterior superior iliac spine with the pubic tubercle. Poupart's ligament served as a reference from which the measuring sites at $5 \mathrm{~cm}$ 's interval on the medial aspect of the sartorial muscle were determined along the projected course of the superficial femoral artery. This methodical preparation assured good orientation, so 
that the time-separated measurements with both instrumentations could be carried out more or less at identical sites.

The examination was usually started with the CW Doppler system and, after completion, repeated with the multi-gate pulsed Doppler instrumentation. This scheme implies a substantial separation in time (30-45 minutes) between measurements with each instrumentation for each site of sampling. The examination was usually concluded with the measurements of the ankle pressures unless the patient was unable to walk.

The measurements with both instrumentations were carried out in the same order, starting with the common femoral artery just below the ligament of Poupart, followed by measurements at the preselected and marked sites in the upper leg and finishing with the popliteal artery with the leg flexed in the knee or with the patient turned on the left side.

The way Doppler signals were selected for further processing was rather similar for both instrumentations in spite of their technical differences. Simultaneous interpretation of the CW audio signal and its corresponding analog velocity signal, displayed on an oscilloscope (fig. 3.2), enabled the examiner to select the optimal signal. The multi-gate pulsed Doppler system offers its user the advantage that velocity measurements may be carried out in readily identifiable arteries. The 32 simultaneously displayed analog signals (section 3.4) allow the examiner good

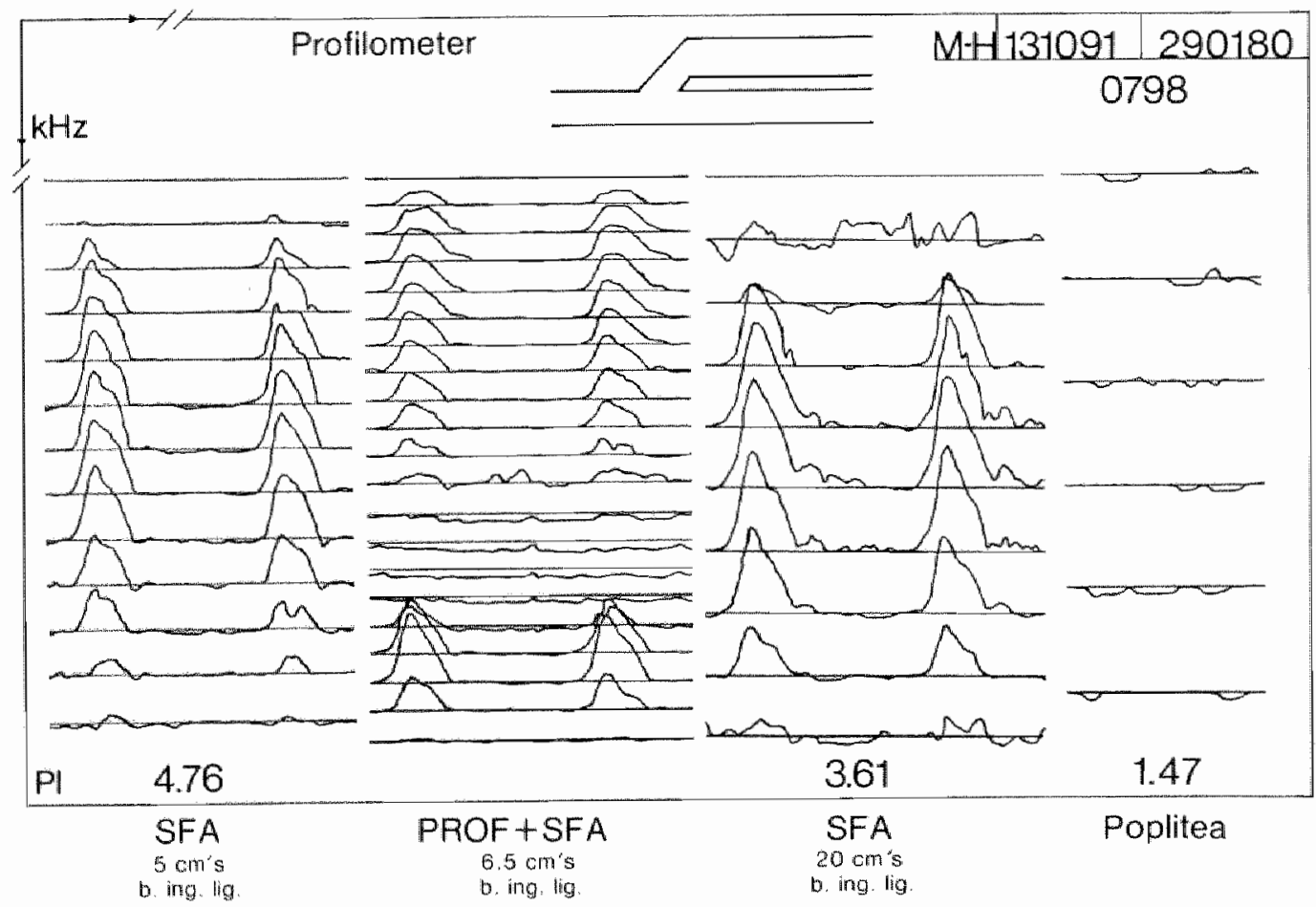

Fig. 3.7A Four recordings are displayed, obtained with the multi-gate pulsed Doppler system, in a patient with an occluded popliteal artery and a slightly dilated SFA (fig. 3.7B). The second recording from the left shows the simultaneously recorded SFA (11 channels) and deep femoral artery ( 4 channels). Both vessels are clearly defined and separated by a vein. The popliteal artery was not found. 
orientation in depth. Having first located and sampled the common femoral artery, identification of the profunda and the superficial femoral artery (SFA) was carried out using the bifurcation (fig. 3.7A, 3.8) and the femoral vein (fig. 3.9) as a guide. The femoral vein is a useful landmark because of its constant position between the superficial femoral and deep femoral arteries, especially helpfull in the case of a full-length occlusion of the superficial femoral artery. Midstream velocity measurements (fig. 3.3) were then carried out in the deep femoral artery distally to the bifurcation and in the SFA at 10,15 and $20 \mathrm{~cm}$ 's below the inguinal ligament. A footswitch controls the selection of the analog signal for further processing by the wave form analyser. Hard copies were made at each site of sampling.

The examination was concluded with the measurement of the ankle systolic pressure index at rest and after exercise. The systolic arterial pressure in the arm (brachial artery) and at the ankle (posterior tibial or dorsal pedal artery) were determined at rest in standard fashion with a semi-automatic sphygmomanometer
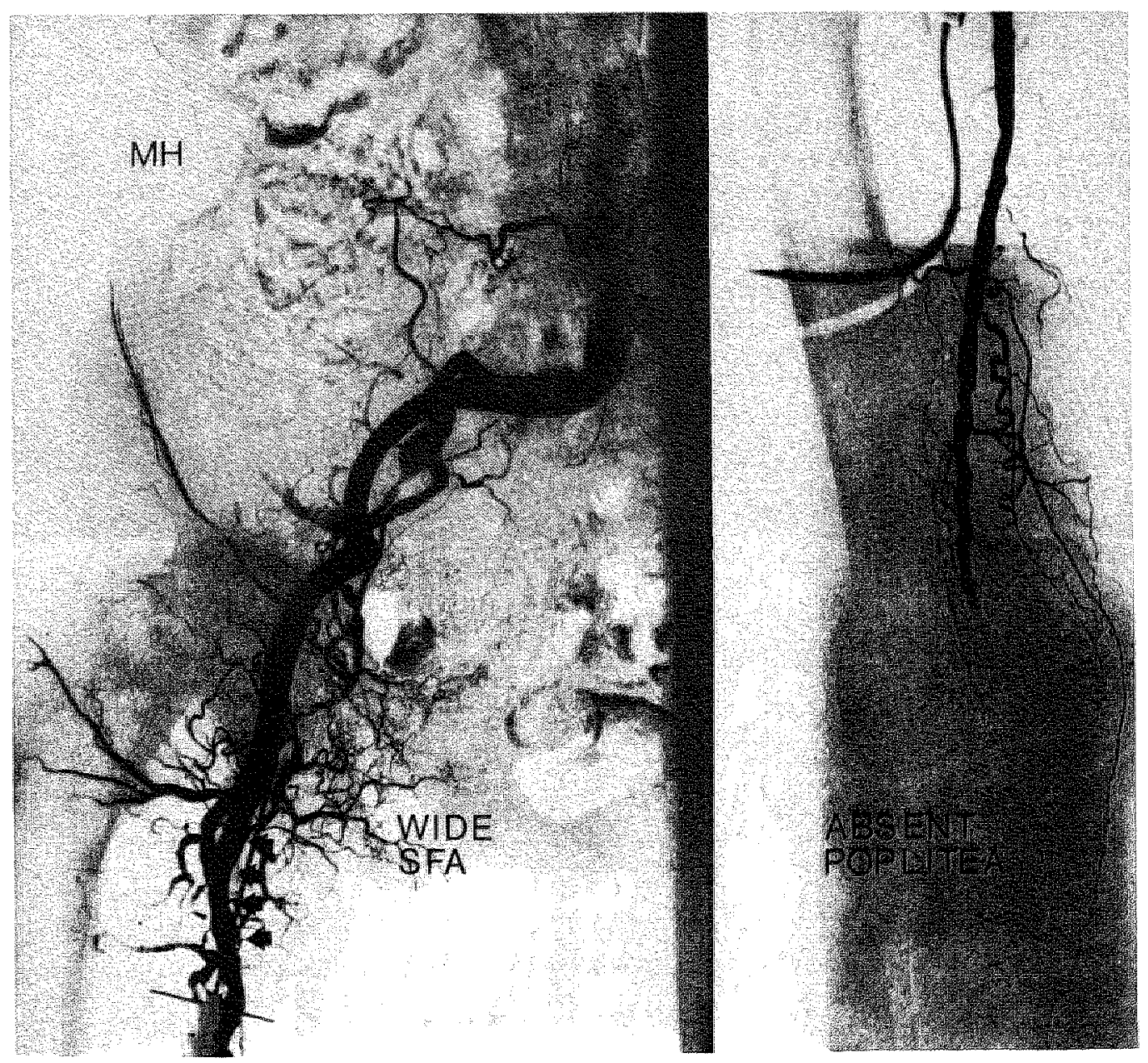

Fig. 3.7B Femoral arteriogram (subtraction) for comparison with the recordings of the multigate pulsed Doppler system of fig. 3.7A. Note wide SFA and occluded popliteal artery. 


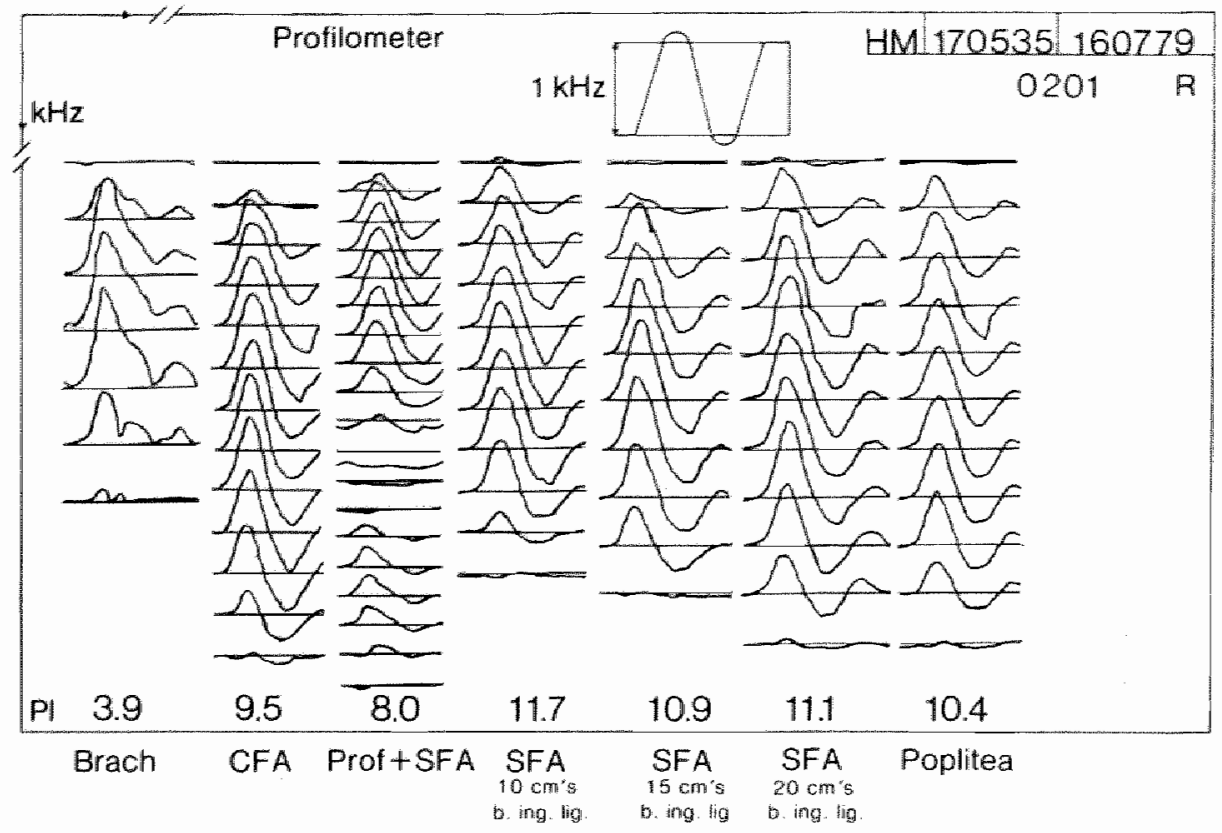

Fig. 3.8 Seven recordings with the multi-gate pulsed Doppler system at seven sites along the arterial segment of interest, are shown in a normal subject. The third recording from the left displays the simultaneously reconded SFA and dleep femoral artery, clearly separated by a vein. Note the increase of pulsatility going downstream.

\section{calfcompression}

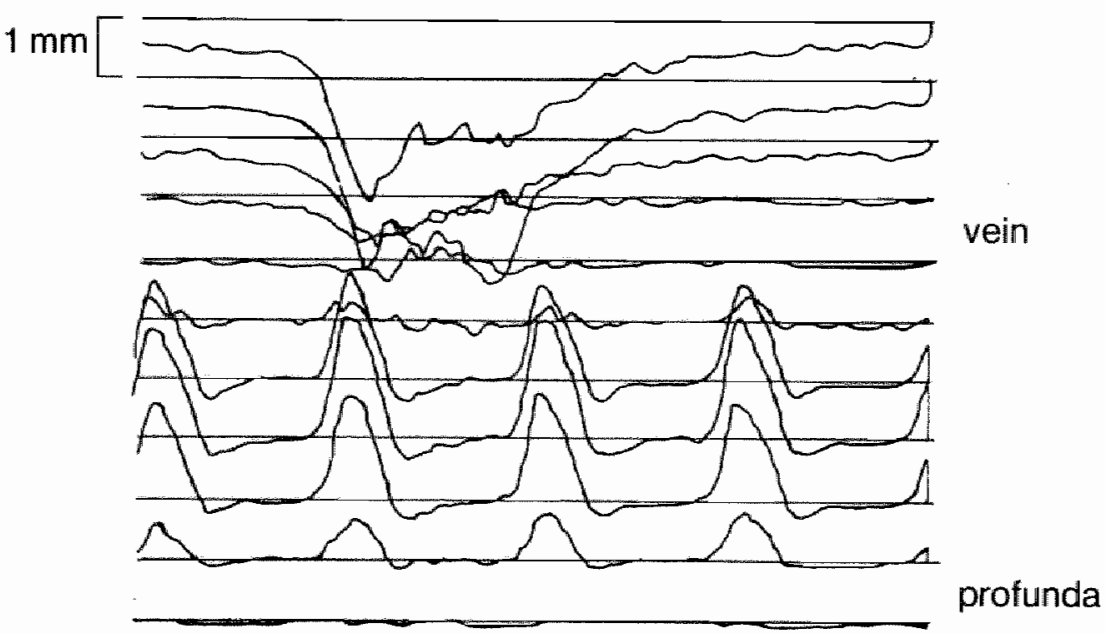

Fig. 3.9 Identification of the deep femoral artery, as shown above in a case of full-length SFA occlusion, is greatly facilitated by the enhancement of venous flow through 'calfcompression'. The femoral vein is thus used as a landmark. 
(cuff size $35 \times 14 \mathrm{~cm}$ ) using the CW Doppler instrument for detection of flow. The patient was then asked to walk on a treadmill in the horizontal plane ( 4 $\mathrm{km} / \mathrm{h}$ ) for 5 minutes or shorter if forced to stop by claudication. After the supine position was resumed (30-45 secs) arm and ankle pressures were recorded at one-minute intervals until the ankle pressure had returned to the pre-exercise level.

\subsection{The off-line data processing, definitions}

A representative velocity-time wave form was selected from the hard copy output of both instrumentations for each measuring site and compiled on large sheets of paper to prevent loss and confusion of data. Underneath each recording the corresponding parameter values were added as sampled with the wave form analyser. Each printed line gave PI, $V_{\max }, V_{\min }, \bar{V}_{(t)}$, and $D_{\text {peak-to-peak }}$ averaged over 4 heartbeats. The mean value of the printed PI and DT measurements were computed off-line with a pocket calculator for all measuring sites, altogether over 20-30 consecutive heartbeats, after elimination of some measurements which were

\section{Multi-gate pulsed Doppler system}

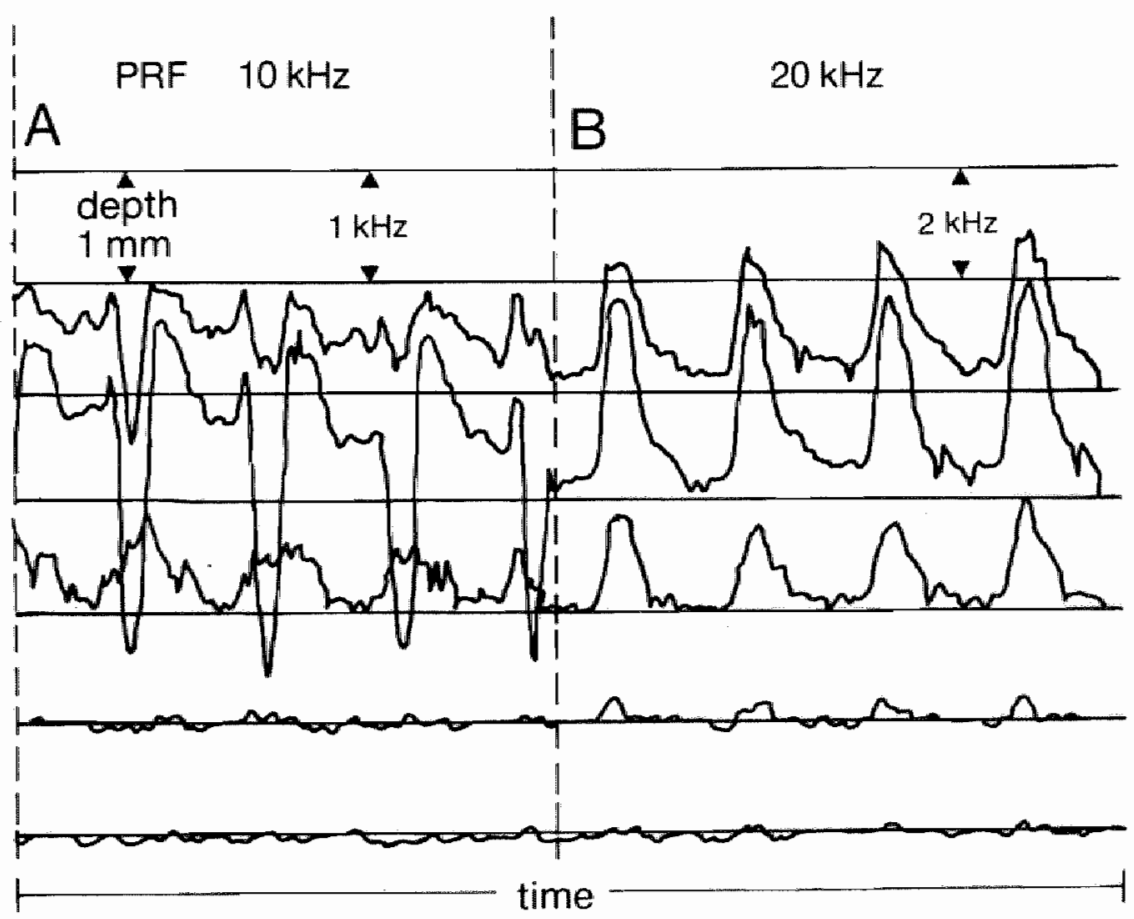

Fig. 3.10 The 'fold-over' of the analog velocity-time wave form, obtained with the multi-gate pulsed Doppler system, and sampled with a pulse repetition frequency (PRF) of $10 \mathrm{kHz}(\mathrm{A})$ in a stenosis of a venous femoro-popliteal bypass at the level of its proximal anastomosis. The bypass at this point is less than $3 \mathrm{~mm}$ in width. The wave form contour was restored to normal when a PRF of $20 \mathrm{kHz}$ was selected (B). The instantaneous mean frequency of the centerstream sample volume was approximately $4 \mathrm{kHz}$ so that $\mathrm{f}_{\max }$ exceeded $5 \mathrm{kHz}$, causing 'fold-over' when sampled with a PRF of $10 \mathrm{kHz}$. 
more than 2 SD's away from this mean value. These averaged values of PI and DT are subsequently used in the study.

The multi-gate pulsed Doppler system is able to provide a hard copy displaying all analog velocity wave forms in 32 channels. The number of actually recorded channels was usually restricted to those showing active engagement with arterial signals in order to save plotting time. They were counted so that noninvasive information was obtained about the width of the vessell segment under investigation for later comparison with the arteriographic information. Finally a qualitative description of the analog velocity wave form of the common femoral artery, as recorded with the multi-gate pulsed Doppler system, was given, differentiating "fold-over" (fig. 3.10A) and 'dicrotic notch' (fig. 3.11A) as well as backflow or diastolic forward flow (fig. $3.10 \mathrm{~B}$ ) in the most centrally situated channel.

All data considered essential were put on punchcards. They comprised information on the patient (section 3.2), the arteriographic material (section 3.3), the pressure measurements from the arm and the ankle (section 3.5) and the hemodynamic parameters as listed in the beginning of this section*. The parameters used for analysis in chapter 4 were stored, labeled and/or calculated with computer-assisted techniques (Statistical Package of Social Sciences, 113). The following parameters were defined:

* Rough data are available on request with the athor.

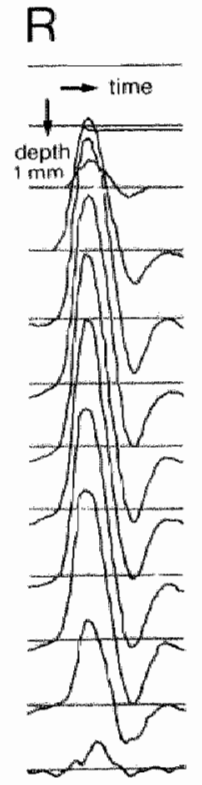

GFA $11 \mathrm{~cm}$

b. ing lig

PI 9.1

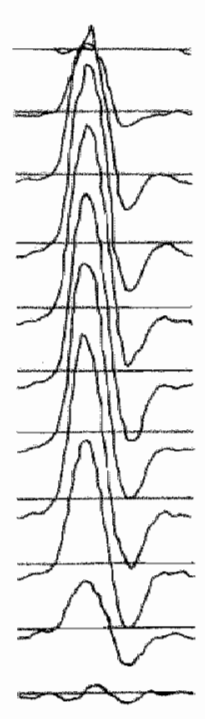

SFA $20 \mathrm{~cm}$ 's b. Ing. lig.

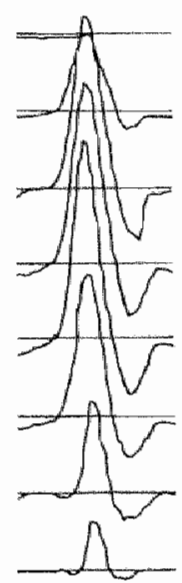

Popliteas artery
$L$
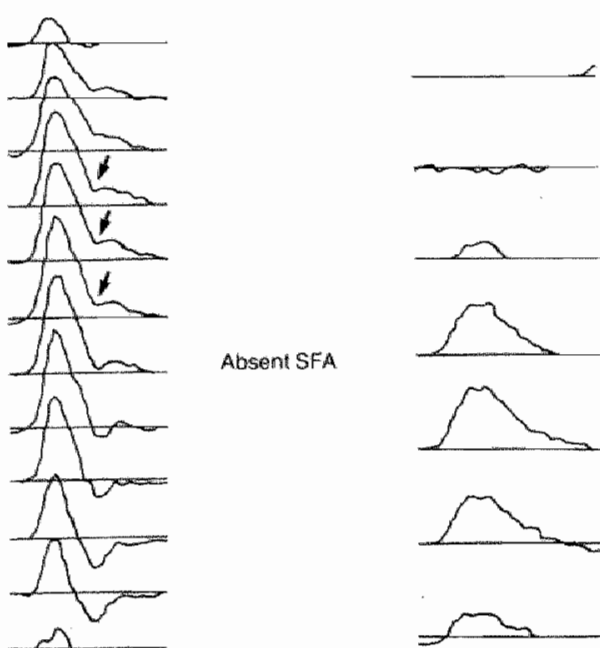

Poplitteall artery 
Fig. 3.11B On the transumbar worto-arteriogram of a man 65 years of age, full information from the renal arteries down to the popliteal artery is giwen. No aortoliac lesions in this single plane arteriogram are observed. Atherosclerotic disease is present in the right SFA (irregularly defined arterial wall) without significantly obstructing arterial now. The left SFA is occluded over its entire leugth from the common femoral bifurcation to the proximal part of the popiliteal artery. The deep femoral artery has a firm caliber. The five multi-gate pulsed Doppler recordings of lig. $3.1 \mathrm{AA}$ were obtained in this pattient.

Fig. 3.11A Five recordings with the multi-gate pulsed Doppler instrument (time-mode) are displayed from a patient with an occluded SFA on the left side (arteriogram (fig. 3.11B). Three recordings, made in the right $\operatorname{leg}(R)$, show nomal velocity signals in the common femoral artery (CFA) over 10 channels $\left(\right.$ Diam $\left._{C F}=10\right)$, in the superficial femoral artery (SFA) at $20 \mathrm{~cm}^{3} \mathrm{~s}$ below the inguinal ligament over 9 channels (DiamsFA-20 $=9$ ) and in the poptiteal artery over 7 channels $\left(\right.$ Diam $\left._{\text {pop }}=7\right)$.

Only two recordings obtained in the left leg (L) are displayed because no welocity signalls were round at $20 \mathrm{~cm}^{3}$ s bellow the in guinal ligament. The velocity-time wave form of the CFA shows the dicrotic "notich" (arrow) in six chanmels nearest to the transducer (transducer top of figure) while the four, deeper situated, channels show velocity wave forms with a nomal configuration. The recording is probably made just proximal to the pro funda bifurcation so that reflected waves do not influence the four deeper situated velocity channels. The popliteal artery of the left leg shows a dampened wave form. The effect of the dicrotic "notch' on the value of PI calculated from the centerstream wave form is evident.

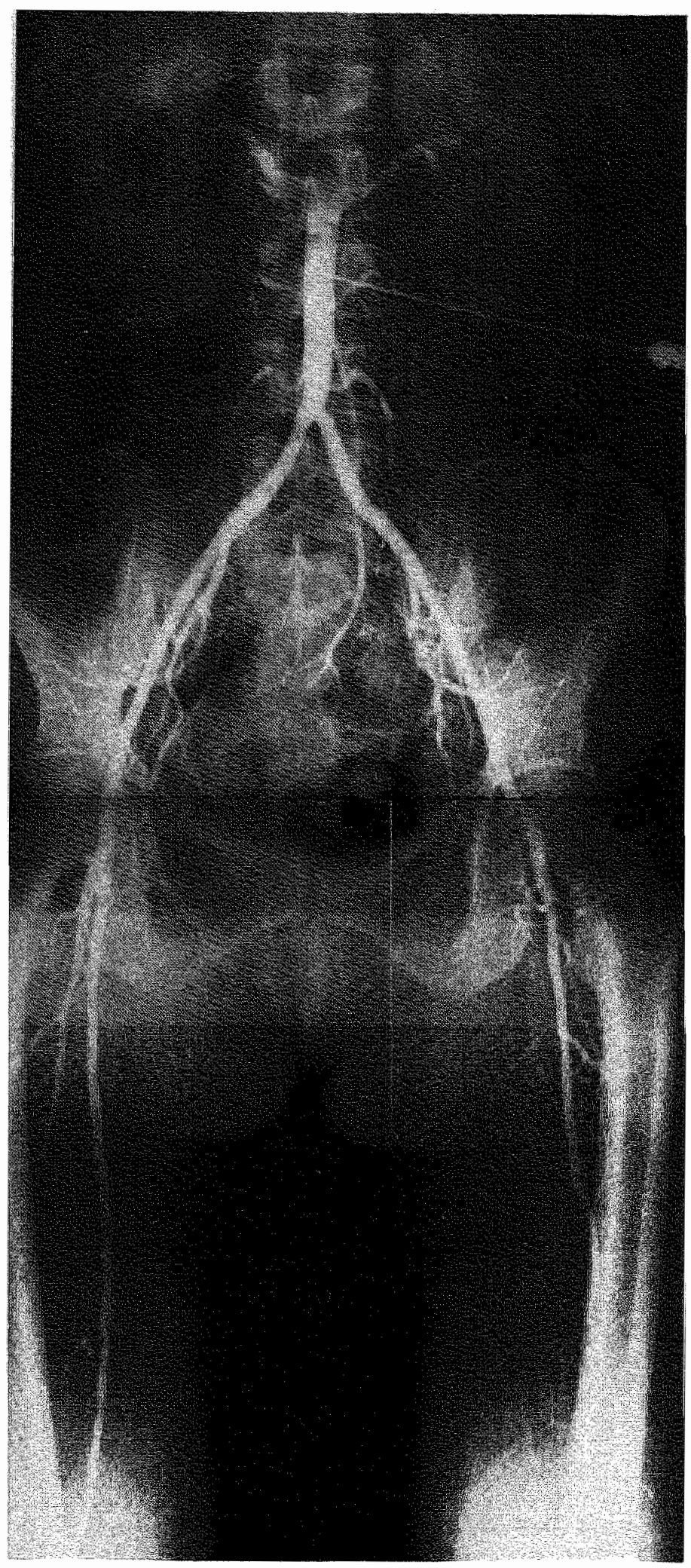


$\mathrm{CW}$ instrumentation:

PI CFA-CW : PI common femoral artery

PISFA-10-CW, PISFA-15-CW, PISFA-20-CW: PI superficial femoral artery at respectively 10,15 and $20 \mathrm{~cm}$ 's below the inguinal ligament

$P I_{\text {pop-CW : }}$ PI popliteal artery

DTCFA-CW: delay-time between the heart and the common femoral artery (DT peak-to-peak)

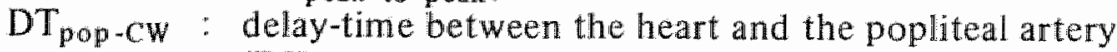
$\left(\mathrm{DT}_{\text {peak - to-peak }}\right)$

Multi-gate pulsed Doppler instrumentation:
PICFA-PD : PI common femoral artery
Plprof-PD : PI deep femoral artery
PISFA-10-PD, PISFA-15-PD, PI SFA-20-PD: PI superficial femoral artery at re- spectively 10,15 and $20 \mathrm{~cm}$ 's below the inguinal ligament
$\mathrm{PI}_{\text {pop-PD }}$ : PI popliteal artery
DTCFA-PD : delay-time between the heart and the common femoral artery (DT $T_{\text {peak-to-peak }}$ )

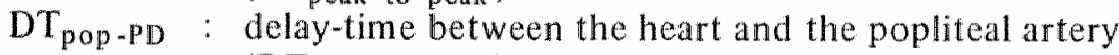
(DT peak-to-peak)

Diam $_{\text {CFA }}$, Diamsfa-10, Diamsfa-15, Diamsfa-20, Diamprof, Diampop: estimated diameter of respectively common femoral artery, superficial femoral artery at 10,15 and $20 \mathrm{~cm}$ 's below the inguinal ligament, deep femoral artery and popliteal artery.

The following parameters were calculated:

- Index $\mathrm{I}=$ ankle systolic pressure at rest/brachial systolic pressure at rest

- Index II = ankle systolic pressure after exercise/brachial systolic pressure after exercise

$-\mathrm{TT}_{\mathrm{CW}}=\mathrm{DT}($ pop-CW $)-\mathrm{DT}_{(\mathrm{CFA}-\mathrm{CW})}\left(\mathrm{TT}_{\text {peak }- \text { to-peak }}\right)$

$-\mathrm{TT}_{\mathrm{PD}}=\mathrm{DT}\left(\right.$ pop-PD)$-\mathrm{DT}(\mathrm{CFA}-\mathrm{PD})\left(\mathrm{TT}_{\text {peak }}\right.$ - io-peak $)$

Transit-time between the common femoral artery (CFA) and the popliteal artery (pop) with the $\mathrm{CW}$ instrumentation ( $\mathrm{TT}_{\mathrm{CW}}$ ) and the multi-gate pulsed Doppler system (TTPD).

$-\Delta$ com-pop $\mathrm{CW}=\mathrm{PI}_{\text {pop-CW }} / \mathrm{PI}_{\mathrm{CFA}-\mathrm{CW}}{ }^{*}$

$-\Delta$ com-pop PD $=\mathrm{PI}_{\text {pop }-\mathrm{PD} / \mathrm{PI}_{\mathrm{Cl}} \mathrm{A}-\mathrm{PD}}$

Damping $(\Delta)$ between the common femoral artery (CFA) and the popliteal artery (pop) with the $\mathrm{CW}$ instrumentation ( $\Delta$ com-pop $\mathrm{CW}$ ) and the multi-gate pulsed Doppler system ( $\Delta$ com-pop PD)

$-\Delta$ prof-pop $\mathrm{PD}=\mathrm{PI}_{\text {pop }-\mathrm{PD}} / \mathrm{PI}_{\mathrm{prof}-\mathrm{PD}}$

Damping $(\Delta)$ between the deep femoral artery (prof) and the popliteal artery (pop) with the multi-gate pulsed Doppler instrumentation.

* The distally measured PI is the numerator and the proximally measured PI the denominator. Femoro popliteal disease is thus indicated with small values $(0$ to 1.0$)$ of this inverse damping factor which is felt to be comformable with current clinical practice where most clinical measurement indices decrease witl increasing disease states $(80)$. 


\subsection{Sources of error and inaccuracy}

\subsubsection{Introduction}

Physiological measurements in patients, using electronical equipment such as described in the preceding sections of this chapter, may suffer from inaccuracies, introduced by a variety of factors, widely different in origin but also in significance. One may distinguish instrumental error and patient- or examiner-related sources of inaccuracy. They may be proportional or non-proportional with the signal value as well as systematic or random.

The cumulative effect of all sources of error or inaccuracy on $P I$ and DT peak-to-peak measurements can be appreciated when descriptive statistical techniques are applied to prolonged PI and DT recordings such as displayed in fig. 3.12. However, no further analysis of these particular frequency histograms is carried out, because identification of the contribution of each source of error or inaccuracy is only feasible in special studies in which all potential sources of error are well controlled. This was considered to be beyond the scope and possibilities of this study.

The discussion in the forthcoming sections is therefore restricted to some widely recognized sources of error and inaccuracy following observations on prolonged on-line recordings of PI and $\mathrm{DT}_{\text {peak -to-peak . }}$

\subsubsection{The signal-to-noise ratio}

The use of a frequency-to-voltage converter, based on the number of zero-crossings per time-unit, requires an adequate signal-to-noise ratio of the input-signal. Taking a directional Doppler signal power $\mathbb{P}_{s}$ with average frequency $\bar{f}_{s}$ and noise with average frequency 0 (relative to the carrier frequency) and power $P_{n}$, then the detected average frequency $\overline{\mathrm{f}}_{\mathrm{d}}$ can be expressed as:

$$
\overline{\mathrm{f}}_{\mathrm{d}}=\frac{\overline{\mathrm{f}}_{\mathrm{s}}}{1+\frac{\mathrm{P}_{\mathrm{n}}}{\mathrm{P}_{\mathrm{s}}}}
$$

showing that for a signal-to-noise ratio of 10 the Doppler frequency will be underestimated by approximately $9 \%$.

Each individual is different with regard to his or her subcutaneous fatlayer, the location of arteries, the absorption of ultrasound in the tissues and the reflection of ultrasound at tissue interfaces, so that signal-to-noise ratio's will vary accordingly. Signals were excluded from further processing if the audio signal and the visual display of the analog signal were of such poor quality, that a low signal-tonoise ratio was suspected although no quantification of the signal-to-noise ratio was attempted. It should be noted, that the error of DT and PI (fig. 3.12) was determined for signals without clearly identifiable superimposed noise.

\subsubsection{The wave form analyser}

The time resolution of peak detection depends on the sample rate of the velocity wave form ( $400 \mathrm{~Hz}$ ), the accuracy of the analog-to-digital converter (ADC), the signal input range (peak-to-peak value), as well as on the frequency content and 


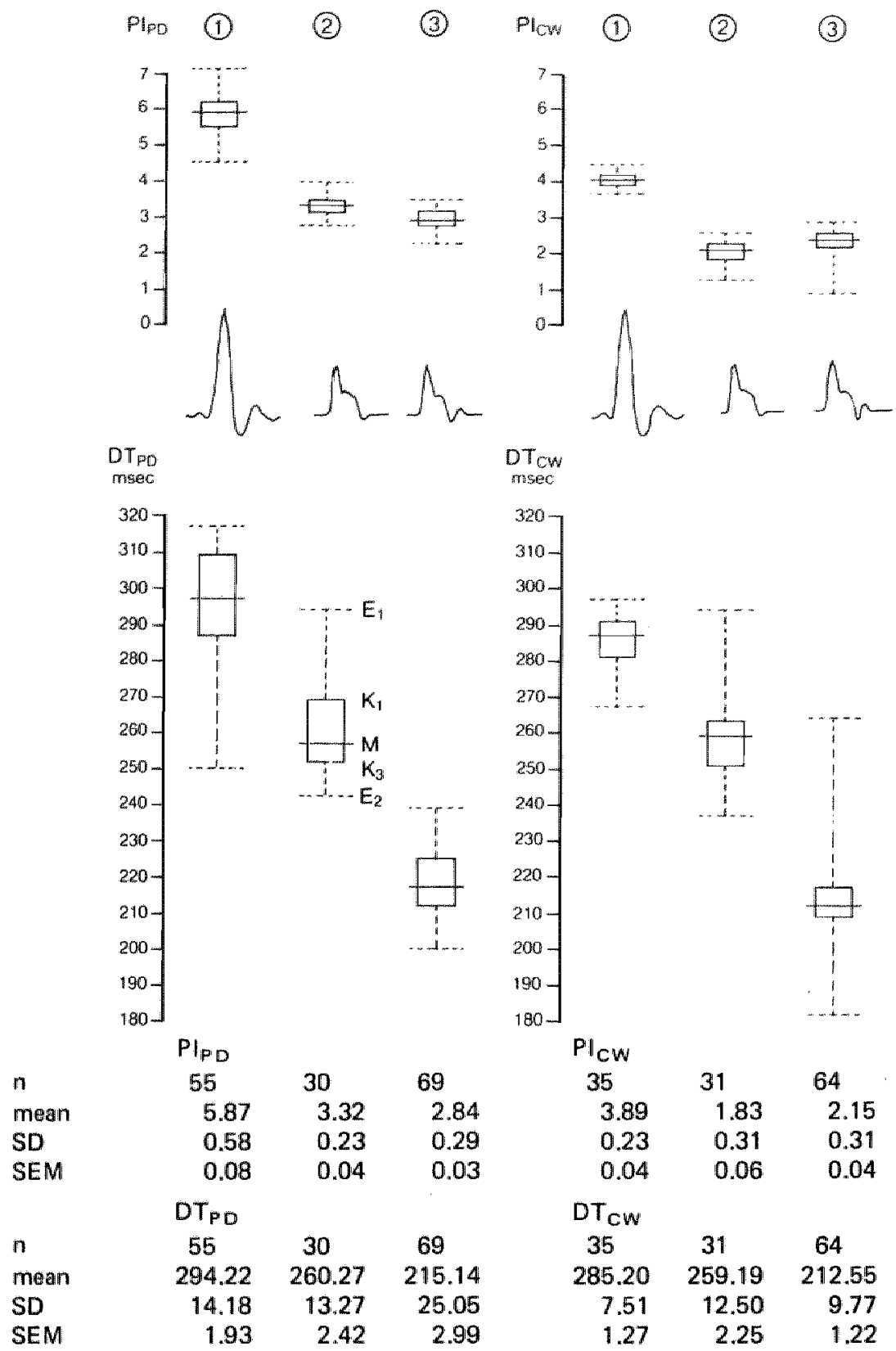

Fig 3.12 Single beat PI and DT measurements from prolonged velocity recordings in the SFA at $20 \mathrm{~cm}$ 's below the inguinal ligament were carried out in three patients with both instrumentations. The analog velocity-time wave form (CW Doppler) is displayed for each of the three patients. The Tukey histograms depict the distribution of $\mathrm{PI}_{\mathrm{PD}}, \mathrm{PI}_{\mathrm{CW}}, \mathrm{DT}_{\mathrm{PD}}$ and $\mathrm{DT}_{\mathrm{CW}}$ over each individual value range. Each histogram has two extreme values $\left(E_{1}\right.$ and $\left.E_{2}\right), 50 \%$ of all parameter values are situated in the column between $\mathrm{K}_{1}$ (quartile one) and $\mathrm{K}_{3}$ (quartile three) and the median value of all recorded values is indicated with $\mathrm{M}$. The Tukey histograms are especially useful for immediate appreciation of skew-ness of a particular distribution. The number of heartbeats (N), the mean and standard deviation (SD) as well as the standard error of the mean (SEM) are calculated for PI and DT for each prolonged velocity recording. 
amplitude of the actual velocity wave form. A Doppler frequency input range of $20 \mathrm{kHz}$, converted by an 8 bit $\mathrm{ADC}$ results in a frequency resolution of $80 \mathrm{~Hz}$ (20.000/256). Assuming the velocity wave form to be a sine wave with an amplitude equivalent to a Doppler frequency of $1 \mathrm{kHz}$ and a frequency of $4 \mathrm{~Hz}$ (corresponding with the natural frequency of the velocity wave in the femoral artery), than the input signal will not vary more than $80 \mathrm{~Hz}$ around the systolic peak value over an interval of $16.6 \mathrm{msec}$. With higher velocities and thus a higher amplitude of the velocity wave form, this time reduction will become better.

The $80 \mathrm{~Hz}$ accuracy of peak detection, in a worst-case situation of $1 \mathrm{kHz}$ input signal (Doppler frequency), determines a $4 \%$ error in the determination of the peak and $8 \%$ in the determination of the peak-to-peak value. The averaging procedure over the cardiac cycle will effectively reduce the error present in the determination of $\bar{V}_{(t)}$. The error in the calculation of PI is therefore virtually the same as with $\mathrm{V}_{\text {peak -to-peak }}(8 \%)$.

\subsubsection{The pulse repetition frequency (PRF)}

One recognized source of error is related to the PRF of the pulsed Doppler system and its accuracy to determine the maximum frequency in the Doppler signal. The Nyquist theorem states that the sample frequency should be at least twice the maximum encountered frequency in a signal. Violation of the Nyquist theorem results in 'fold-over' of the wave form peak, introducing error in the measurement of DT and PI. This phenomenon is often observed under high velocity conditions such as encountered in a stenosis (fig. 3.10 and 4.3). If recognized in time, a higher PRF can be selected to comply with the Nyquist theorem. Small distortions in the analog wave form, especially in combination with poor signal-to-noise ratio's are difficult to detect and quantify.

\subsubsection{Patient-and examiner-related sources of inaccuracy}

The beat-to-beat variations in PI, calculated from an analog tracing with an adequate signal-to-noise ratio, display a regular pattern if followed over a prolonged period of time (example A, fig 3.13). Although PI is theoretically independent of the angle of insonation, these variations can be the result of small angle changes during the cardiac cycle owing to respiration-induced changes in the position of the probe during the on-line recording. Pulsed Doppler instruments, having a small sample volume in both the lateral as well as the axial direction, suffer from the additional disadvantage that displacement of the sample volume in the axial direction occurs following these small alterations of position even if the angle of insonation is maintained. This may explain the somewhat larger beat-tobeat fluctuations as shown in example B (fig. 3.13). Venous contamination cannot be responsible for these variations because the velocity samples are obtained centerstream in the artery.

Another source of variation in PI over prolonged periods of time can be the irregular heart rate of the patient. Example $C$ (fig 3.13), however, shows that in this case the influence of cardiac arrhythmia on PI is surprisingly small, presumably because beat-to-beat differences affect peak-to-peak and mean velocity values equally. Acute step-wise changes in $\mathrm{PI}$ are also observed during continuous recording, probably resulting from sudden movements of the hand caused by examiner's fatigue or anxiety of the patient (example D, fig 3.13) 


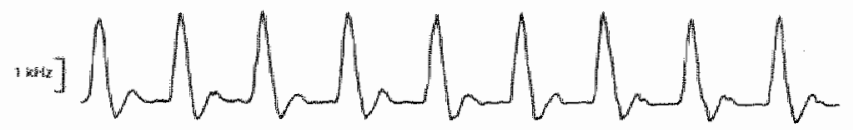

(B)

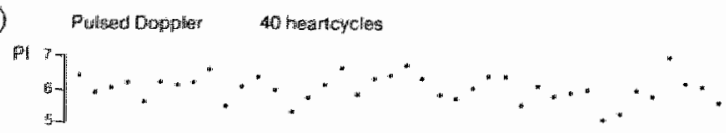

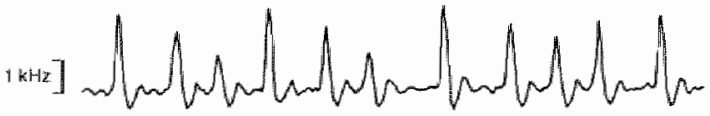

(D)

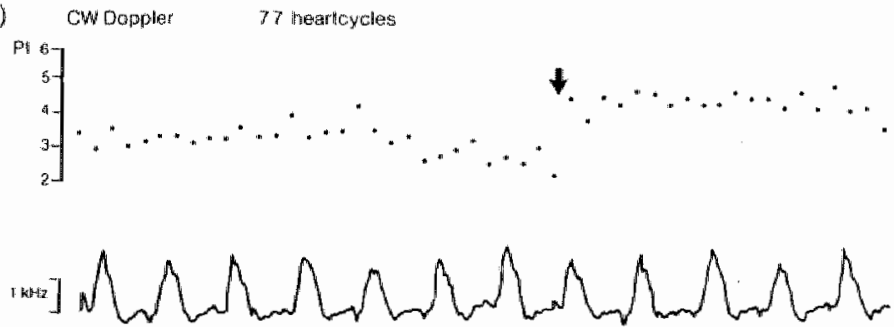

Fig. 3.13 (A, B) Prolonged on-line PI recording in SFA $20 \mathrm{~cm}$ 's below the inguinal ligament in a healthy young man with the CW Doppler instrument (A) and the pulsed Doppler system (B). Both recordings show a regular respiratory-induced, pattern of variation in the value of PI. Note the difference in beat-to-beat variation caused by the different modes of sampling.

(C) Prolonged on-line PI recording with the $\mathrm{CW}$ instrumentation in man of 73 years with atrial fibrillation, in the SFA at $20 \mathrm{~cm}$ below the ligament of Poupart.

(D) Prolonged on-line PI recording with the $\mathrm{CW}$ instrumentation in man of 61 years. Note sudden change in PI value $(\downarrow)$ during this period of uninterrupted recording. For explanation $\mathrm{M}, \mathrm{K}_{1}, \mathrm{~K}_{3}, \mathrm{E}_{1}, \mathrm{E}_{2}$ see figure 3.12 .

\subsubsection{The cumulative effect from all sources of error and inaccuracy}

The error introduced by the velocity wave form analyser has a rectangular probability density function so that the range of error in the worst-case situation per single heartbeat amounts to $16.6 \mathrm{msec}$ for $\mathrm{DT}_{\text {peak-to-peak }}$ and $8 \%$ for PI. The maximum variation of $\mathrm{PI}$ and $\mathrm{DT}\left(\mathrm{E}_{1}-\mathrm{E}_{2}\right)$ observed during prolonged recordings ( $30-60 \mathrm{sec})$ with both instrumentations is shown in fig. 3.12. This variation is far in excess of $16.6 \mathrm{msec}$ for DT and $8 \%$ for PI so that other sources than the veloc- 
ity wave form analyser must be responsible. It should be realized that in this study mean values of PI and DT peak-to-peak are used, produced by averaging over 20-30 consecutive heartbeats, so that the error attributable to the wave form analyser is reduced to less than $4 \mathrm{msec}$ for DT $\left(\frac{16.6}{\sqrt{25}}\right)$ and $1-2 \%$ for PI $\left(\frac{8}{\sqrt{25}}\right)$. The examples given in fig. 3.13 suggest that patient-and examiner-related sources of inaccuracy (respiration, cardiac arrhythmia, step-wise changes), in combination with variable levels of noise, contribute significantly to the shown variations. Any attempt, however, to specify the importance of each source of error and inaccuracy must end in speculation because they are probably interrelated. Older patients with arterial disease are often difficult to examine and the signal-to-noise ratio of Doppler signals obtained distal to occluded arteries is relatively poor in general.

\subsection{Statistical methods}

\subsubsection{Discriminant analysis}

This study is designed to evaluate a score of variables, which may or may not be interrelated. The classical statistical tool for the management of such problems is discriminant analysis, one of the many possible forms of multivariate analysis. The mathematical objective of discriminant analysis is to weigh and combine the discriminating variables - in this study the hemodynamic parameters - in such a fashion that the groups are forced to be as statistically distinct as possible. In this study, only linear combinations of the discriminating variables were used. This type of analysis is basically an attempt to maximize statistical separation through the formation of 'discriminant functions" which take the general form:

$$
D_{i}=d_{i 1} Z_{1}+d_{i 2} Z_{2}+\ldots d_{i p} Z_{p}
$$

where $D_{i}$ is the score on the discriminant function, the $d_{i}$ 's are weighting coefficients and the $Z$ 's are the standardized values of the $p$ discriminating variables used in the analysis.

The underlying statistical theory regarding the calculation of the coefficients of these discriminant functions is beyond the scope of this study and described in detail elsewhere $(98,153)$.

The number of functions, produced by the analysis, is closely related to the number of groups or discriminating variables under study. In general, the maximum number of discriminant functions to be computed is either one less than the number of groups or equal to the number of discriminating variables, whichever is smaller. This study distinguishes between three arteriographic groups - normal, stenosis and occlusion - so that two discriminant functions suffice to describe the multi-dimensional space formed by the parameters. The discriminant functions can be thought of as the axes of a geometric space allowing the evaluation of spatial relationships between these groups. The computation of more than two functions is theoretically possible, but practically of no value, because most of the discriminating power in the parameters is already picked up by the first two functions. The importance of each function in this respect is given by the Eigen- 
value, which is a quantification of the discriminating power removed by each discriminant function.

The standardized discriminant function coefficients are of great analytical value, because they allow identification of parameters contributing most to the observed differentation along the respective dimension (= function). The larger the numerical value of a particular standardized coefficient, the greater the contribution of the associated parameter to the discrimination between the three arteriographic groups. In general not all the parameters will give a significant contribution to the discrimination between the groups.

In addition to the described analysis technique, discriminant analysis also provides the opportunity to evaluate the classifying properties of the parameters. Analysis is concerned with the identification of the best discriminating parameters, taking into consideration all cases simultaneously. Classification, on the other hand, makes use of the calculated functions and coefficients in reverse order, its success empirically measured by observing the proportion of cases successfully classified (à posteriori) into their correct previously defined group (à priori).

In section 4.9 several examples of the computer output, concerning the a posteriori classification, are given. All legs included in the analysis are à priori labelled with their group membership, their à posteriori group placement in the scattergram is determined by both discriminant functions. Legs trespassing the limits of a mathematically defined space (territory) are considered incorrectly classified legs. They can be identified on the scattergram(s) (fig. $4.24 \mathrm{~A}$ and B, fig. 4.25) although accurate information on the percentages of correctly classified legs is also supplied per group, with a table (table 4.8).

\subsubsection{Other statistical tools}

Additional statistical procedures used in this study are the Mann-Whitney test (113) for the determination of the degree of statistical difference between groups, and linear regression, with its associated Pearson coefficient of correlation. 


\section{Results and discussions}

\subsection{Introduction}

The following eight sections present the results of this study. Each titled section is completed by a short discussion.

\subsection{Comparison of PI and DT as obtained with both Doppler instrumentations}

\subsubsection{Results}

The measurements with both instrumentations, carried out according to the protocal (section 3.5), permitted comparison of mean PI and DT values as obtained with both Doppler instrumentations at each arterial site in the upper leg. Differences between the corresponding parameter values can have a systematic or non-systematic appearance. Systematic differences are likely to be associated with differences in the technical design and sampling properties of both instrumentations, and they can be evaluated to some extent with linear regression. Nonsystematic differences, on the other hand, are more likely to be the result of the many unpredictable and often interrelated sources of error and inaccuracy such as described in section 3.7. The significance of these instrument-related (signal-tonoise ratio, wave form analyser) and patient- or examiner-related errors and variations can be established to some extent with correlation studies. However, sudden stepwise changes in the parameter value such as displayed in fig. 3.7 will interfere with the interpretation of these correlation studies because their random appearance and magnitude have considerable influence on the averaged value (20-30 consecutive heartbeats) of the hemodynamic parameter, used for correlation. The most obvious stepwise changes were off-line removed from the 5-8 series of 4-heartbeats, available at each site of measurement. This preliminary consideration in mind, no serious objections against correlation studies of mean Pl and DT values were felt to remain because the averaging procedure over so many heartbeats (20-30) results in a mean value determination of sufficient accuracy (note SEM, fig. 3.12).

The Pearson coefficient of correlation and the coefficients of the regression lines were computed for the corresponding PI and DT measurements as obtained with both instrumentations at six arterial sites and summarized in table 4.1. PIPD and $\mathrm{PI}_{C W}$ correlate poorly especially in the higher PI ranges, causing the shape of the correlation diagram (fig. 4.1) to be of triangular form. The regression line, calculated from paired observations for each arterial site except the deep femoral artery, showed a positive intercept and a slope of less than $45^{\circ}$. The corresponding DT measurements (DT $T_{\mathrm{PD}}$ wS $\mathrm{DT}_{\mathrm{CW}}$ ) correlated more evenly over the whole 
Table 4.1 The Pearson coefficient of correlation ( $R$ ) and the coefficients of the regression lines were calculated for each pair of PI (PIPD and PI $I_{C W}$ ) and DT (DT PD and DT $\mathrm{CW}_{\mathrm{P}}$ ) values, ob. tained at six arterial sites.

$S F A_{10}, S F A_{15}, S F A_{20}$ : superficial femoral artery at respectively 10,15 and $20 \mathrm{~cm}$ 's below the inguinal ligament. Each value of PI or DT used in the correlation diagram is the averaged value of 20.30 consecutive heartbeats.

\begin{tabular}{lcccc}
\hline $\begin{array}{l}\text { Pulsatility Index } \\
\left(\mathrm{PI}_{\mathrm{PO}} \text { and } \mathrm{PI}_{\mathrm{CW}}\right)\end{array}$ & R(Pearson) & Intercept & $\begin{array}{c}\text { Direction } \\
\text { coefficient }\end{array}$ & No. of legs \\
\hline Brachial artery & 0.68 & 1.39 & 0.75 & 282 \\
Common femoral artery & 0.77 & 1.50 & 0.79 & 278 \\
SFA 10 & 0.77 & 1.35 & 0.86 & 254 \\
SFA 15 & 0.78 & 1.36 & 0.89 & 237 \\
SFA 20 (see fig. 4.1). & 0.80 & 1.61 & 0.81 & 224 \\
Popliteal artery & 0.78 & 1.42 & 0.77 & 272 \\
& & & & \\
Delay-Time & & & & 248 \\
(DTPD and DT & & & & \\
\hline Brachial artery & 0.79 & 62 & 0.74 & 226 \\
Common femoral artery & 0.82 & 54 & 0.82 & 219 \\
SFA 10 & 0.92 & 37 & 0.89 & 201 \\
SFA 15 & 0.92 & 42 & 0.87 & 240 \\
SFA 20 (see fig. 4.2) & 0.91 & 36 & 0.89 & \\
Popliteal artery & 0.88 & 34 & 0.91 & \\
\hline
\end{tabular}

range of their values, while also consistently showing a positive intercept with a slope of less than $45^{\circ}$ (fig. 4.2 ).

\subsubsection{Discussion}

The decreasing correlation as noted with the higher values of PI is unlikely to be associated with the error related to poor signal-to-noise ratio's, since they occur especially in the presence of arterial disease and consequently in Doppler signals with low PI values (section 3.7.6). This suggests that patient-and examiner-related sources of inaccuracy are mainly responsible for the relatively low degree of correlation shown at the higher end of the value range.

The pulsatility of the velocity signal is usually large in healthy arteries, so that one may assume that PI in those cases is calculated from small-banded high frequency Doppler signals. The Nyquist theorem regarding the sampling of signals demands that the PRF used in the pulsed Doppler system (sections 2.9 and 3.7.4) should be twice the maximum frequency occurring in a velocity signal.

The Doppler frequencies occurring with higher tow velocities are sometimes in the order of $10 \mathrm{kHz}$ with the use of a $6 \mathrm{MHz}$ Doppler instrument (fig. 4.3). Prominent wave form distortion ('fold-over' in fig. 3.10,4.3) occurs when a PRF of $10 \mathrm{kHz}$ is selected, waming the examiner to switch over to a PRF of $20 \mathrm{kHz}$. However, smaller distortions may go unnoticed resulting in appreciable under-

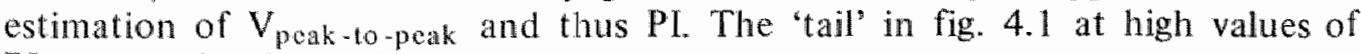
Plcw may thus be explained.

No definite conclusions can be arrived at with the results of regression analysis 


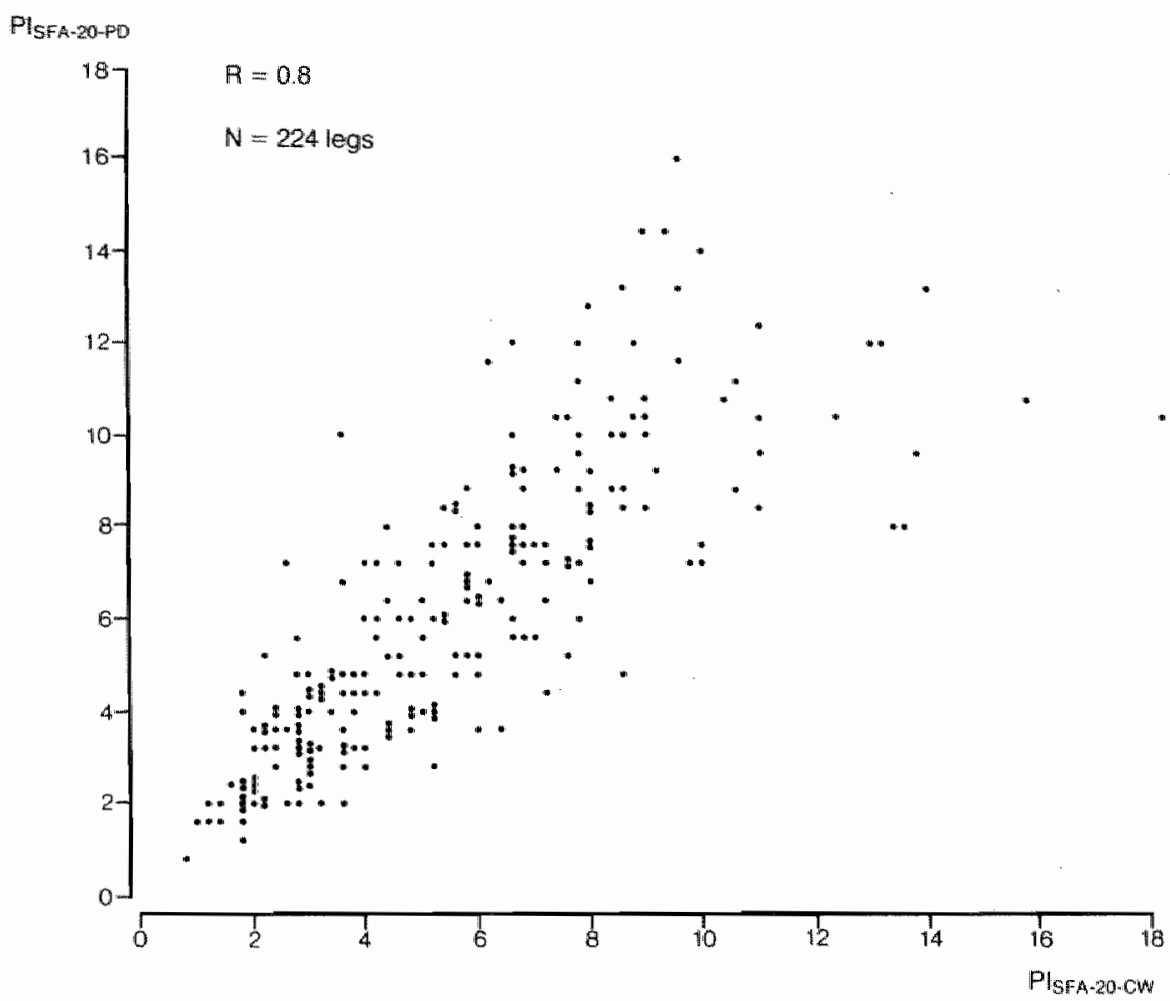

Fig. 4.1 In this example $\mathrm{PI}_{\mathrm{PD}}$ and $\mathrm{PI}_{\mathrm{CW}}$, obtained in the superficial femoral artery at $20 \mathrm{~cm}$ 's below the inguinal ligament are compared in a correlation diagram. Note the triangular shape and 'tail'.

(table 4.1) owing to the poor correlation between $\mathrm{PI}_{\mathrm{PD}}$ and $\mathrm{PI}_{\mathrm{CW}}$. One has to bear in mind that the PI when determined with the pulsed Doppler system is more sensitive to changes in the transducer positioning than with a CW system. Even if one assumes that the sample volume remains coincident with the centerline of the artery throughout the cardiac cycle, unpredictable differences between the corresponding PI values occur owing to their dependence on the velocity profile. This unpredictability is related to differences in the time-dependent velocity profile as observed between individual patients as well as between various intraarterial sites of sampling especially in the presence of disease at the site of measurement.

The observed degree of correlation between $\mathrm{DT}_{\mathrm{CW}}$ and $\mathrm{DT} \mathrm{T}_{\mathrm{PD}}$ appears to be independent of the incident value of DT (fig. 4.2). The R-wave of the ECG is considered to be a stable point of reference so that variations in the measurement of DT refer to problems encountered in the detection of the systolic peak of the velocity wave form.

The duration of systole in the arteries of the leg, assuming a normal myocardial contraction, is approximately $250 \mathrm{msec}$, so that random displacement of the incident peak may occur to a maximum of $125 \mathrm{msec}$. Therefore if the error related to the wave form analyser is added, $16 \mathrm{msec}$ in the worst-case situation (see section 3.7.3), a beat-to-beat variation of $141 \mathrm{msec}$ is possible. This single beat variation of DT is reduced to approximately $28 \mathrm{msec}$ by averaging over $20-$ 


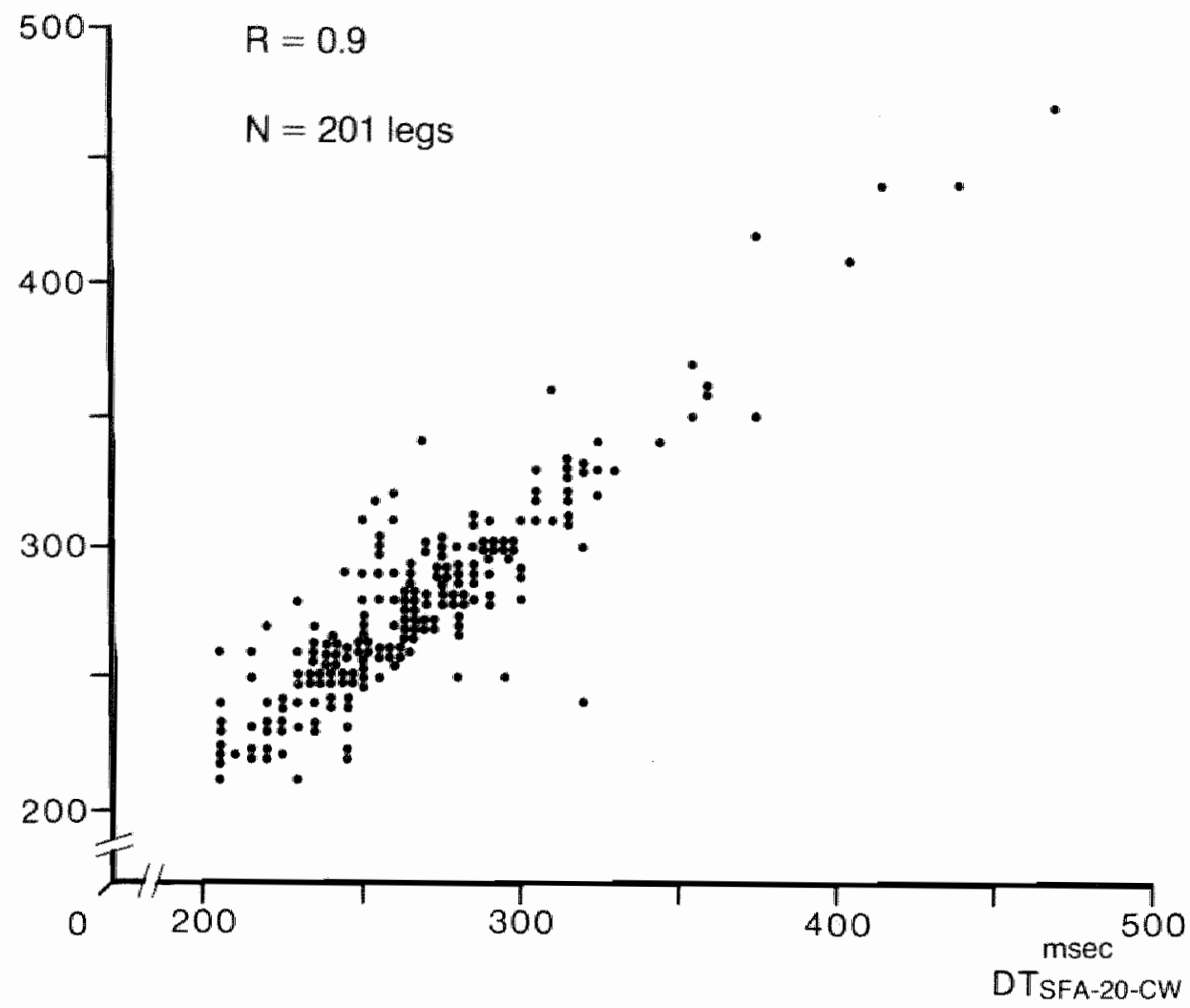

Fig. 4.2 In this example DT $P D$ and $D T_{C W}$ obtained in the superficial femoral artery at $20 \mathrm{~cm}$ 's below the inguinal ligament are compared in a correlation diagram.

30 consecutive heartbeats $\left(\frac{141}{\sqrt{25}}\right)$. Poor signal-to-noise ratio's as associated with higher DT values and arterial disease, as well as "fold-over" of the systolic peak, such as observed in the analog wave form of the pulsed Doppler system at higher velocity rates, will be associated with a maximum error of $28 \mathrm{msec}$. The correlation diagram (fig. 4.2) shows that most of the scatter around the regression line is lying within the range of $56 \mathrm{msec}$. This scatter, unlike the scatter as seen with PI, could be attributed theoretically to instrument-related error. This suggests that patient- and examiner-related sources of variation are less significant in the measurement of DT.

It remains doubtful whether linear regression is valuable when applied to correlation diagrams, such as provided by the measurement of $D T_{C W}$ and $D T_{P D}$, because the positive intercept is the result of considerable extrapolation. The direction coefficients of the regression lines computed for the DT measurements (table 4.1) possibly suggest a small systematic delay $(30-60 \mathrm{msec})$ in the arrival of the systolic peak when measured with the pulsed Doppler system. Some of this delay could be explained by the low cutt-off frequency of the low-pass filter ( 7.5 $\mathrm{Hz}$ ) necessary to 'smooth' the velocity signal after zero-crossing in the pulsed Doppler system. Blunting of the velocity wave form results, following loss of 
high frequency components, thus contributing to the delay in the arrival of the systolic peak.

An additional, but theoretical, possibility explaining a systematic difference between $\mathrm{DT}_{\mathrm{PD}}$ and $\mathrm{DT}_{\mathrm{CW}}$, is given by the particular sample characteristics of both Doppler systems in combination with the behaviour of viscous fluids in elastic tubes. McDonald (102) estimated the phase lag between the axial and mural laminae in the femoral artery of a dog to be in the order of $50 \mathrm{msec}$. The $\mathrm{CW}$ system will average this phase lag over the vellocity profile, so that the phase lag between the velocity signals of both Doppler systems will be reduced to 20-30 msec. This hemodynamic factor can contribute to the positive intercept of fig. 4.2 only if the velocity profile is parabolic, which is probably very seldom the case under normal conditions in larger arteries (section 2.8). The multi-gate pulsed Doppler recordings of a normal 45-year old person (fig. 3.8) did not exhibit this phase lag to a noticeable degree.

\subsection{The ultrasonic estimation of the arterial diameter}

\subsubsection{Results}

A preceding pilot-study left the impression that the ultrasonically estimated diameter of an artery corresponded well with the degree of narrowing seen on an arteriogram (fig. 4.3, 4.4, 4.6A and B). Each sample volume along the ultrasonic beam of the pulsed Doppler system has a width of $1 \mathrm{~mm}$, so that the total number of channels in which velocity signals can be detected is representative for the diameter of the vessel if corrected for the angle of insonation. The decision whether to include or exclude a channel during off-line study of the hard copies was often difficult because of the low velocity of blood in the vicinity of the vessel wall. The ultrasonic method is therefore subject to error, for which reason the word 'estimation' is preferred to 'measurement'.

The narrowest point of six predetermined arterial segments in all available arteriograms was measured with a micrometer. The narrowest diameter was measured because it was considered to be representative for the extent of the disease in this particular segment. If a total occlusion was present somewhere in the segment, the diameter was considered zero mm's.

To evaluate the accuracy of these ultrasonically estimated diameters, comparison was made with the arteriographically measured diameters in six arterial segments. Three diameter estimations obtained in the upper leg at three levels below the bifurcation of the common femoral artery (Diam $\mathrm{SF}$ - 10, Diam $\mathrm{SF}$ - 15 ,

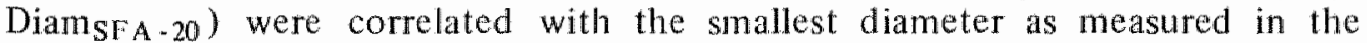
proximal part of the superficial femoral artery. Similar correlations were made in the common femoral, the deep femoral and the popliteal arteries. The Pearson coefficient of correlation was calculated together with the coefficients of the regression line for each of the six arterial sites. The results are summarized in table 4.2 .

The best correlation was found between the ultrasonic estimation and the arteriographic measurement in the superficial femoral artery $20 \mathrm{~cm}$ 's below the inguinal ligament (fig. 4.5). The thirty-two perfectly correlated cases (total occlusion on the arteriogram and absent velocity recording with the pulsed Doppler system) 

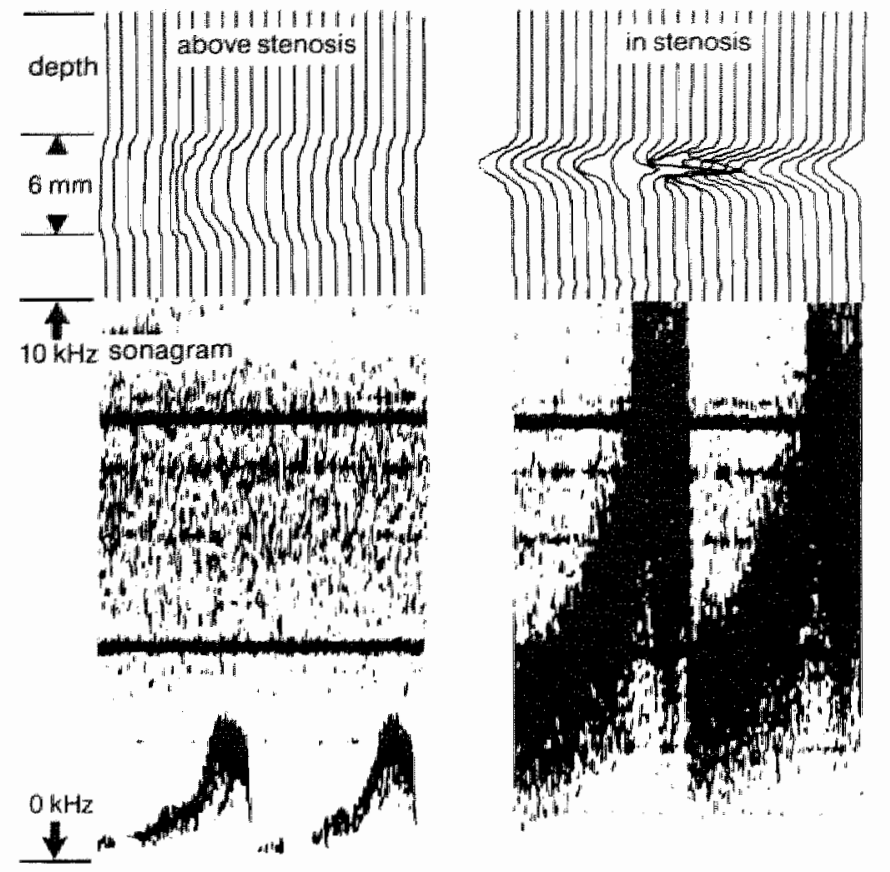

Common temoral antery

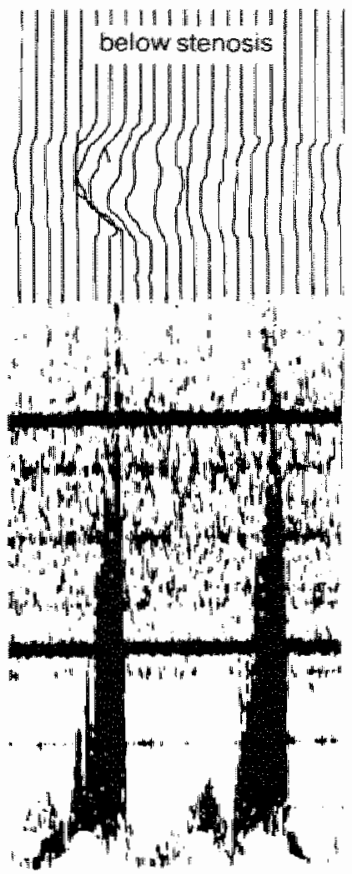

Fig. 4.3 Above, in and below a stenosis in the common femoral artery, velocity profiles are recorded with the multi-gate pulsed Doppler system (depth mode). The sonagram of each centerstream obtained velocity sample is displayed at the bottom of the figure. It may be noted that the maximum centerstream velocities when recorded in the stenosis exceed $10 \mathrm{kHz}$, causing 'fold-over' of the pulsed Doppler signal. The same sonagram, taken centerstream within the stenosis, shows spectral broadening, while frank turbulence is visable in the sonagram dista] to the stenosis. The width of the common femoral artery above, in and below the stenosis corresponds well with its arteriographic visualization in fig. 4.4

undoubtedly had great effect on the high degree of the correlation $(\mathrm{R}=0.8)$. 'Twelve legs could be considered 'falsely' classified. In six legs with an occluded proximal SFA (arteriographic diameter), a positive identification of the SFA at $20 \mathrm{~cm}$ 's below the inguinal ligament was made with the pulsed Doppler system ("false positive"). In six other legs the SFA was patent on the arteriogram but not localized with the multi-channel pulsed Doppler system ('false negative'). When the false classifications were removed from the analysis $R$ increased to 0.9 . The resullts of the statistical analysis after removal of "false negative" and "false positive" findings of each diameter correlation is also summarized in table 4.2, because the corrected $\mathrm{R}$ is indicative for the degree of correlation of arteria] segments apart from occlusions.

A systematic overestimation of the ultrasonically determined diameter was observed in the SFA of 10,15 and $20 \mathrm{~cm}$ 's below the inguinal ligament, caused by the oblique insonation of the artery. If the temptation to calculate the angle of insonation from the corrected directional coefficient (1.26) was not resisted, it appeared that the transducer on the average was held with an angle of $60^{\circ}$ to the arterial axis (fig. 4.5). 


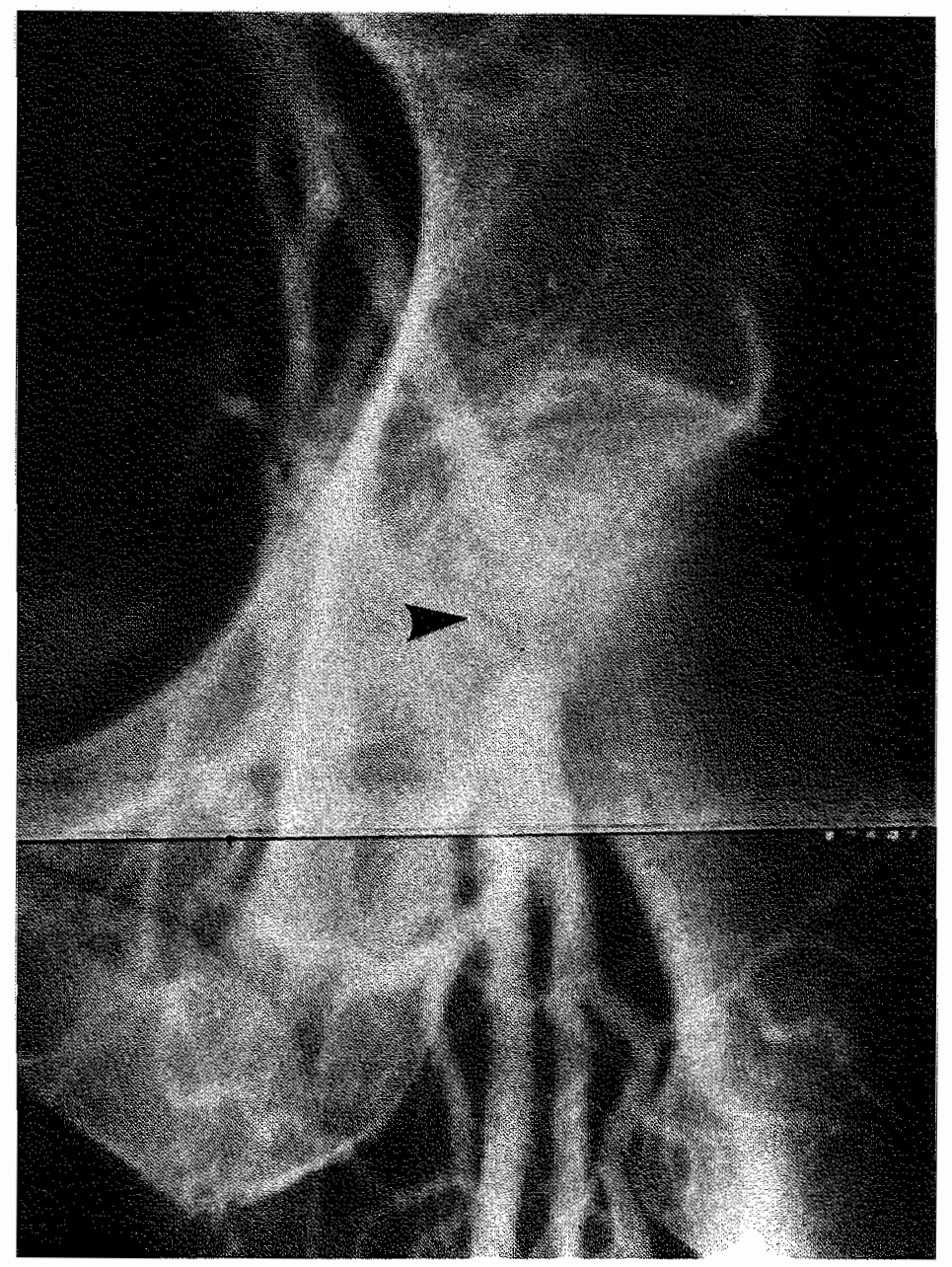

Fig. 4.4 Detail from a translumbar aorto-arteriogram showing a stenosis (arrow) in the common femoral artery one $\mathrm{cm}$ above its bifurcation. The artery was investigated with the multi-gate pulsed Doppler system above, in and below the stenosis (fig. 4.3).

Table 4.2 The Pearson coefficient of correlation (R) and the coefficients of the regression lines were calculated for the paired estimation (multi-gate pulsed Doppler system) and measurement (on arteriogram with a micrometer) of the arterial diameter at six arterial sites.

$\mathrm{SFA}_{10}, \mathrm{SFA}_{15}, \mathrm{SFA}_{20}$ : superficial femoral artery at respectively 10,15 and $20 \mathrm{~cm}$ 's below the inguinal ligament. Between ( ) the results after removal of 'false positive' and "false negative" classifications.

\begin{tabular}{lllll}
\hline & R (Pearson) & Intercept & $\begin{array}{c}\text { Direction } \\
\text { coefficient }\end{array}$ & No. of $l e g s$ \\
\cline { 2 - 5 } Cormmon femoral artery & $0.54(0.44)$ & $3.83(3.93)$ & $0.74(0.73)$ & $147(144)$ \\
Deep femoral artery & $0.33(0.27)$ & $3.66(3.83)$ & $0.36(0.32)$ & $157(155)$ \\
SFA 10 & $0.65(0.81)$ & $3.10(1.24)$ & $0.76(1.14)$ & $158(134)$ \\
SFA 15 & $0.78(0.89)$ & $1.51(0.58)$ & $1.03(1.22)$ & $154(136)$ \\
SFA 20 (see fig. 4.5) & $0.81(0.90)$ & $0.81(0.51)$ & $1.09(1.26)$ & $158(146)$ \\
Popliteal artery & $0.52(0.56)$ & $3.05(1.98)$ & $0.65(0.90)$ & $154(142)$ \\
\hline
\end{tabular}




\subsubsection{Discussion}

The degree of correlation such as found between the ultrasonically estimated and arteriographically measured diameters of arteries in the upper leg is remarkably good if the following considerations are taken into account.

There is no way to be sure that the correlated diameters (ultrasonic vs arteriographic) were indeed obtained from identical locations in the artery which is important because caliber changes of several $\mathrm{mm}$ 's over small distances along a diseased superficial femoral artery are often seen. Mismatch of localization is certainly responsible for some of the scattering around the regression line. Mismatch of localization may be also considered in the case of the six 'false positive' legs (fig. 4.5) because the proximal SFA can be partially occluded, with a patent vessel remnant close to the common femoral artery at the site of the ultrasonic measurement (fig. 4.6 A and B). The six "false negative' legs are obviously true errors. Nevertheless, one felt justified to remove all falsely classified

\section{Diameter}

SFA-20

(nô of channels)

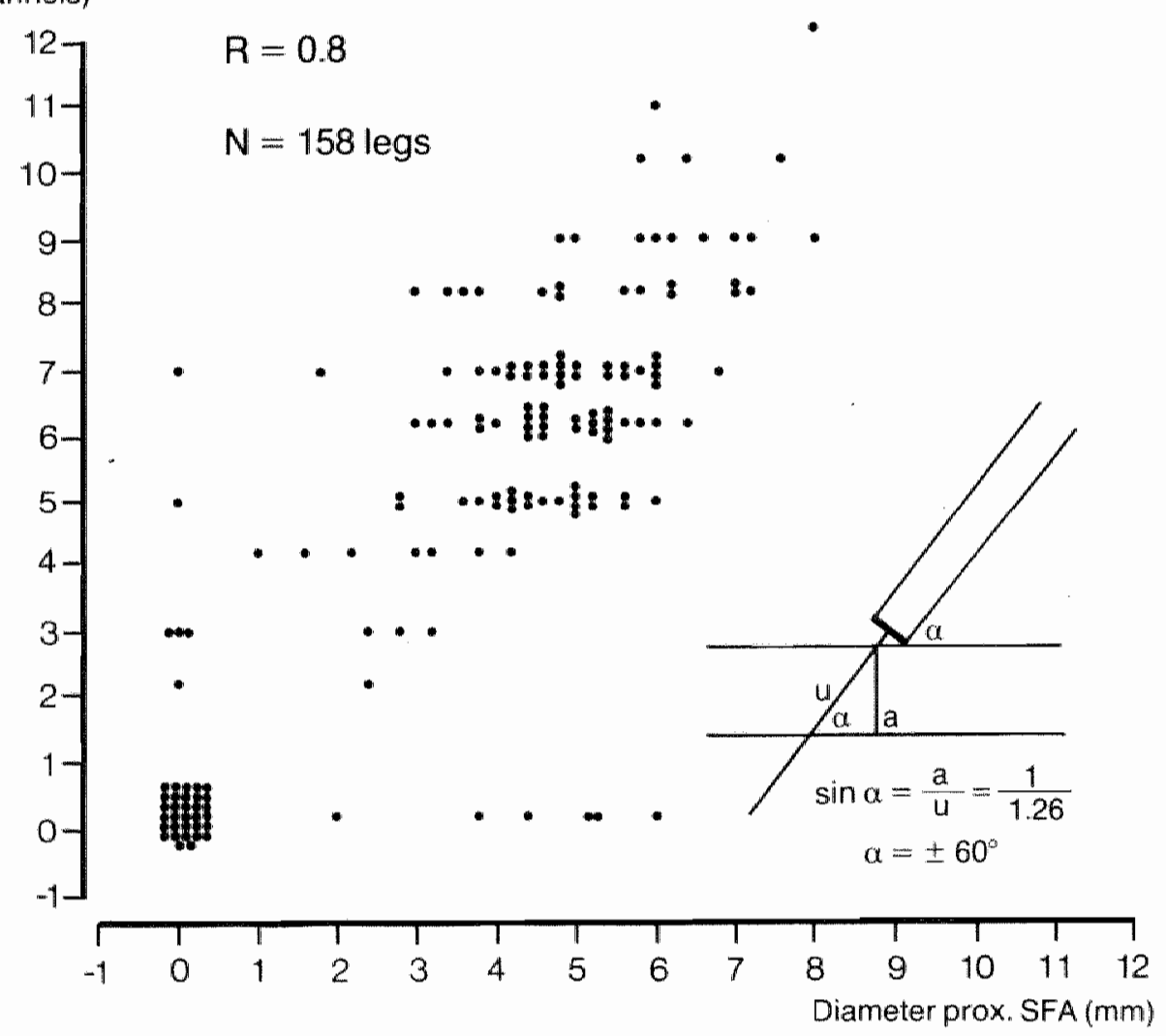

Fig. 4.5 The diameter of the superficial femoral artery (SFA) at $20 \mathrm{~cm}$ 's below the inguinal ligament is estimated with the multi-gate pulsed Doppler system ( $u=$ no. of channels in mm's) and compared with the measured transverse diameter of the proximal part of the SFA. $(a=$ $\mathrm{mm}$ 's). The average angle of insonation $(\alpha)$ is calculated. 
legs if the interest was focussed on the true correlation of measured and estimated diameters of stenosed or unstenosed arteries.

The sagittal and lateral angles of insonation have considerable influence on the actual value of the ultrasonically estimated diameter of an artery. The lateral angle can be controlled to some extent by searching for axis-symmetrical velocity profiles during the patient's examination, which indicates that the ultrasonic beam is aimed at the center of the vessel. This is difficult in practice, so that underestimation of the diameter, thus determined, can occur. Although it is easy to maintain an angle of $45^{\circ}$ to $60^{\circ}$ in the sagittal plain over the superficial femoral artery during prolonged measurements, no certainty exists concerning the angle of insonation regarding more inaccessible arteries like the deep femoral artery and the popliteal artery.

Finally, the diameter measurements of an artery with a micrometer can also be a source of error $(7,39)$. A representative diameter is often difficult to select, especially in arteries with a constantly changing caliber (e.g. the deep femoral artery), and many arteries show poor visual resolution on the arteriogram in the presence of arterial disease (e.g. the popliteal artery).

The ultrasonic estimation of the arterial diameter provides useful and relevant clinical information in spite of the discussed limitations. This is well illustrated with the given examples (fig. 3.7 A and B, fig. 4.6A and B, fig. 4.3 and 4.4 . as well

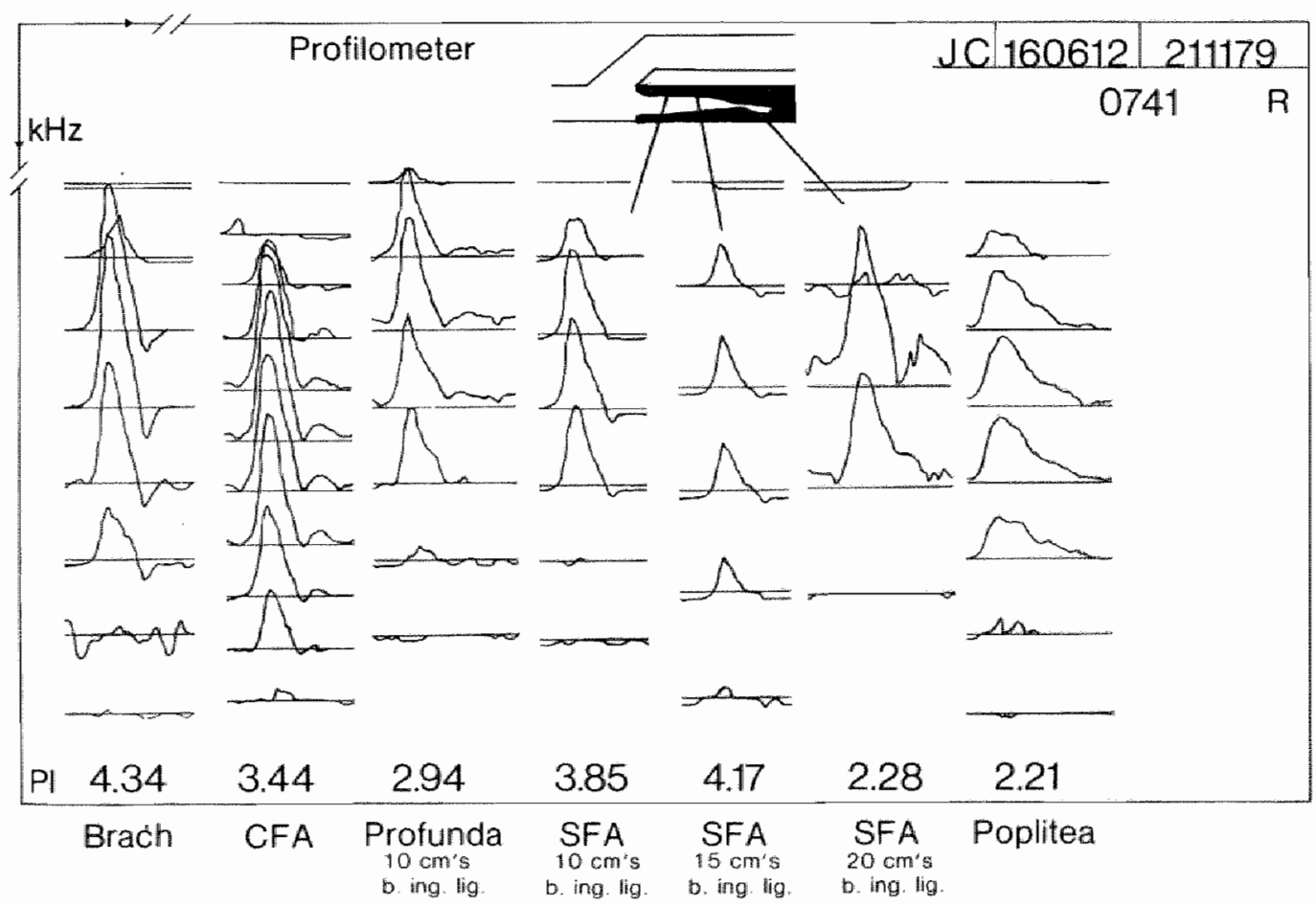

Fig. 4.6A Seven recordings are displayed, obtained with the multingate pulsed Doppler system, from a patient with an occluded SFA. The most proximal part of the SFA is arteriographically patent (fig. $4.6 \mathrm{~B}$ ). The velocity recordings of the $S F A$ at 10,15 and $20 \mathrm{~cm}$ 's below the inguinal ligament show a narrowed arterial segment $\left(\right.$ diam $\left._{S F A-10}=4 ; \operatorname{diam}_{\mathrm{SFA}-15}=4 ; \operatorname{diam} \mathrm{MFA}_{\mathrm{A}-20}=2\right)$. 
as in fig. 3.11A and B). An occluded or patent SFA is diagnosed by the absence or presence of arterial velocity signals as indicated on the display visualizing the 32 channels (fig. 3.11 A and B). If no arterial signals were detected in the expected channels, the assumption that the SFA was occluded appeared to be correct in $72 \%$ of the cases as judged from the arteriograms. If the six 'false positive' legs (mismatch) are excluded, the accuracy of the multi-gate pulsed Doppler system for the diagnosis of an occlusion in the SFA is $84 \%$ (sensitivity). This indicates that the femoral vein, used for orientation (fig. 3.9) during the examination, is a reliable 'landmark' for the separation of velocity signals coming from the deep and superficial femoral arteries. The deep femoral artery was identified with the multi-gate pulsed Doppler system in 155 out of 157 cases, where arteriography had shown a patent vessel (table 4.2).

The multi-gate pulsed Doppler instrument under study appears to estimate the diameter of an artery rather accurately. No other detailed clinical studies regarding multi-gate and arteriographically measured diameters are available. All previous reports $(8,143)$ dealing with Doppler diameter measurements, employ some kind of velocity imaging.

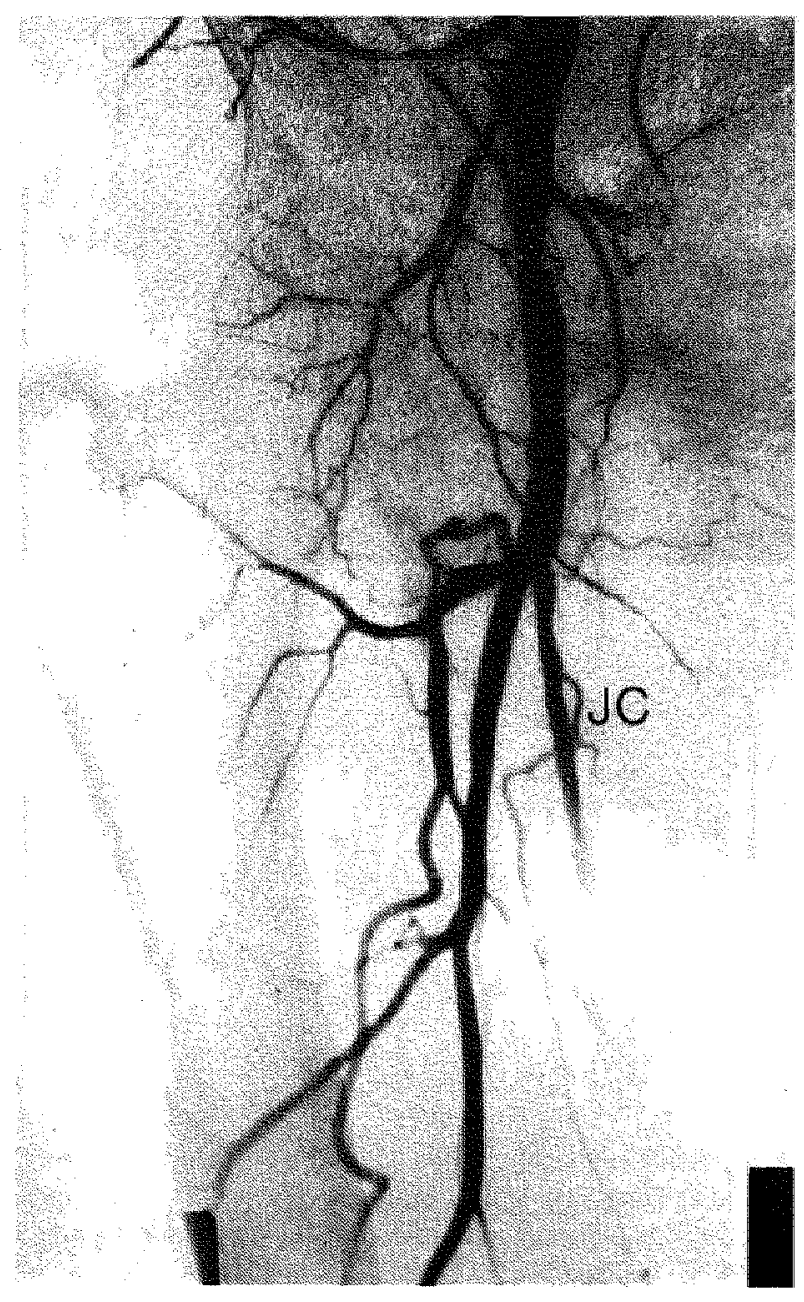

Fig. 4.6B Detail from a femoral arteriogram (subtraction) for comparison with recordings of the multi-gate pulsed Doppler system of fig. 4.6A. Note occlusion of SFA and general narrowing of its most proximal part. 


\subsection{The ankle systolic pressure index}

\subsubsection{Results}

Depending on the presence or absence of arteriographically significant lesions in the aorto-iliac and femoro-popliteal regions, six groups of legs were distinguished (table 4.3. and fig. 4.7). Arteriographic information, from the distal part of the aorta down to the distal part of the popliteal artery was complete in each leg of groups 1 to 5 . Legs with incomplete arteriographic information and a pathological ankle systolic pressure index - ASPI $<0.95$ either at rest or after exercise (group 6) - were also included to show that their functional impairment was equally distributed over the value range of Index I and Index II. Some of these legs are used in later sections.

If the ankle pressure indices were normal at rest and after exercise $(29 \%)$, no significant atherosclerotic lesions were assumed to be present, thereby considerably enlarging the number of legs available for analysis in group 0 (also discussed in sections 3.2 and 3.3 ).

The six groups (0-5) of legs are also used for analysis in section 4.5 . The range of interest in that section is determined by the localization of the proximal and distal ultrasonic measurements, so that the distal part of the popliteal artery, $1 y$ ing distally to the distal ultrasonic measurement, is excluded. One is aware that their exclusion introduces some error in the analysis as described here, but it leaves the main reasoning intact because the number of significant atherosclerotic lesions in the distal part of the popliteal artery is small (table 3.3). It is generally accepted that lesions in the lower leg arteries can remain undetected with the noninvasive measurement of the ankle pressure $(34,149)$ and that their arteriographic appearance can be misleading, which was the reason they were excluded as well.

The determination of the ASPI has great diagnostic value for the differentiation between normal and diseased (fig. 3.1 and 4.7). However, their inability to differentiate legs with disease at different levels is also shown. The pressure indices of legs with single-level aorto-iliac stenosis (group 1) are within the same (wide) range as legs with stenotic lesions in the SFA (group 4). On the other hand, the cumulative effect of multi-level disease on the ASPI evident.

Table 4.3 List of codes for six groups of legs with disease at one or two levels (1-5). Group 6 comprises a group of legs in which no arteriographic information was availlable with either a pathological ASPI at rest or after exercise. Aorto-iliac = aorta + common and external illiac arteries + common femoral artery; SFA = superficial femoral artery from the bifurcation of the common femoral artery to the distal part of the popliteal artery.

\footnotetext{
$0=$ normal

$1=$ aorto-iliac stenosis

2 = aorto-iliac stenosis + SFA stenosis

$3=$ aorto-iliac stenosis + SFA occlusion

$4=$ SFA stenosis

$5=$ SFA occlusion

$6=$ no arteriography and abnormal pressure indices
} 
None of the legs with arteriographically visualized atheroscle rotic lesions in the aorto-iliac and femoral pathways had normal resting and post-exercise pressures. Eight legs with stenotic lesions (critical stenosis) and one leg with an occluded SFA would have been erroneously considered normal on the strength of their normal resting pressure index (marked legs in groups 1, 4 and 5). Statistical confirmation of the diagnostic value of both indices to detect significant disease was obtained by discriminant analysis (section 3.8.1). Correct assignment into normal (0) or diseased (1-5) was present in $99 \%$ of the legs.

\subsubsection{Discussion}

The presented findings sustain the generally held opinion $(34,36,81,147,174)$ that ankle pressures reliably indicate the presence or absence of disease, especially if the pressure after exercise is determined $(34,36,79)$. The nearly total overlap between the pressure ranges of the arteriographic groups with disease of increasing severity is comparable with the results of other studies $(36,81,142)$, and precludes any deductions regarding the localization of disease. That the pressure may drop $10 \%$ in normal subjects after exercise is also previously shown (79, 142).

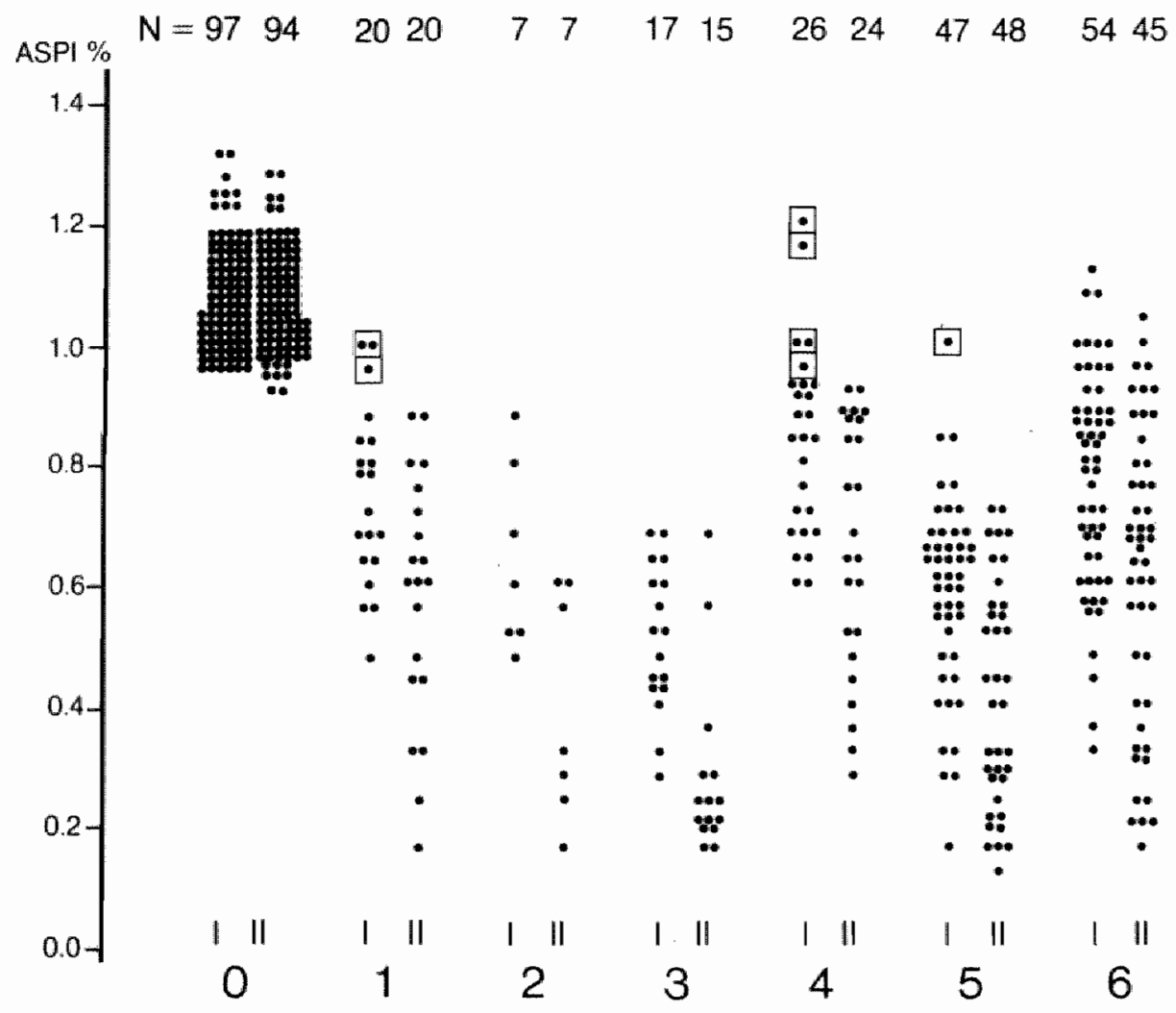

Fig. 4.7 The ankle systolic pressure index (ASPI) at rest (Index I) and after exercise (Index II) are vertically shown for each leg in all arteriographic groups as denoted in table 4.3. The legs of groups 1.4 and $5(\square)$ would have been considered normal on the strength of Index $\mathbb{I}$. 
4.5 Are TT and damping, as well as the arterial diameter estimation of the SFA, determined in the femoro-popliteal pathway, independent of aorto-iliac lesions?

\subsubsection{Results}

If the hemodynamic parameters obtained by measurements in the femoropopliteal pathway are specific for lesions in this region, their independence of aorto-iliac lesions should be made certain.

The TTpeak-to-peak (msec) of the velocity wave in the femoro-popliteal pathway was measured with both instrumentations and is graphically represented in fig. 4.8 for six groups of legs as defined in table 4.3. Aorto-iliac lesions do not seem to influence TT measured in the femoro-popliteal pathway as may be deduced from the similar TT range of groups 3 and 5 as well as of groups 2 and 4 . This was verified with the Mann-Whitney test (table I, appendix) showing no statistically significant differences between the mean TT value of legs with an

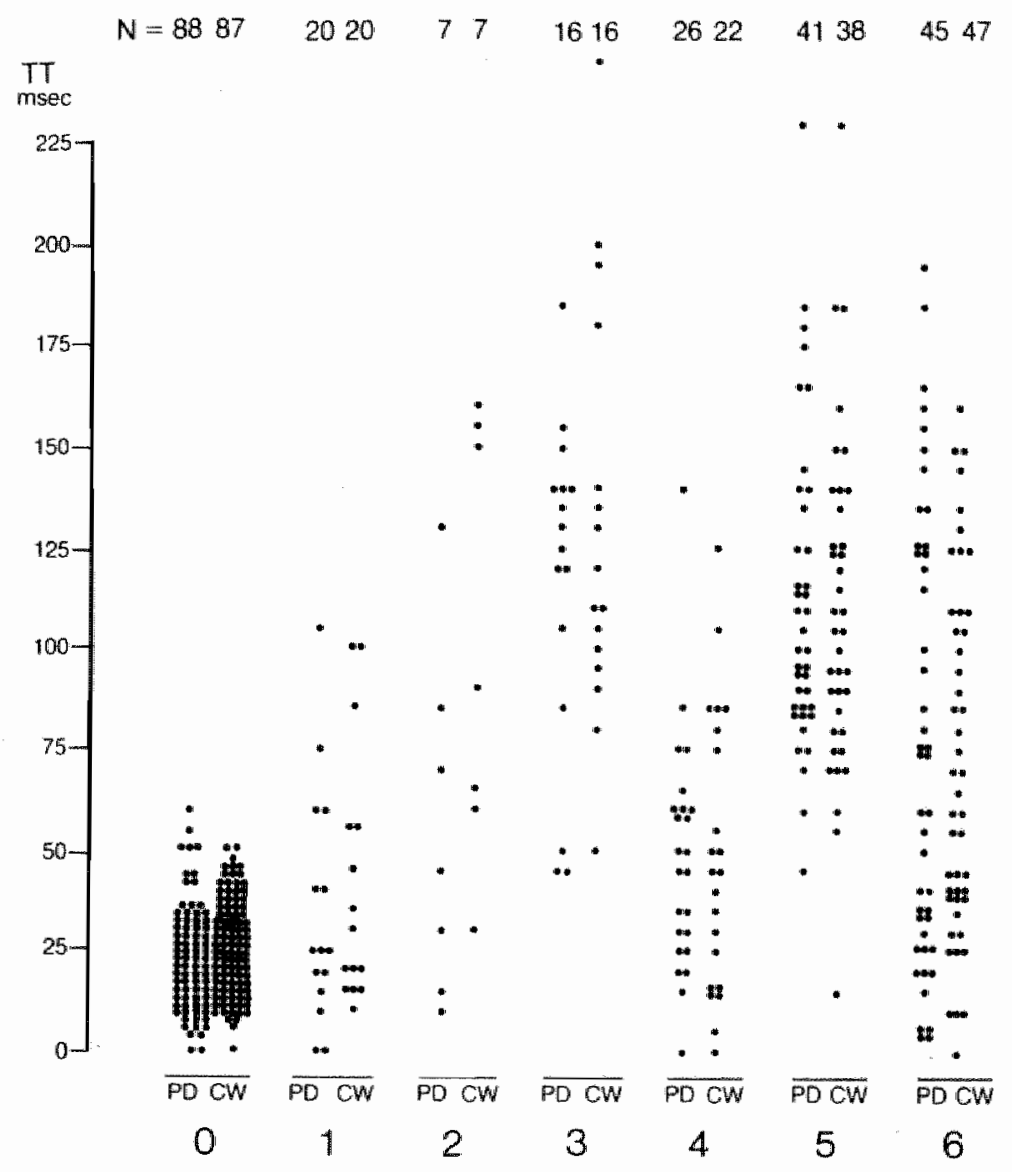

Fig. 4.8 The transit-time ( $\mathrm{TT}_{\text {peak-to-peak }}$ in msec) of the velocity wave between the common femorall artery and the popliteal artery is shown for each leg in all arteriographic groups as denoted in table 4.3. $\mathrm{TT}_{\mathrm{PD}}$ and $\mathrm{TT}_{\mathrm{CW}}$ are pairwise presented for each group and indicated respectively as $\mathrm{PD}$ and $\mathrm{CW}$ at the foot of each column. The number of legs $(\mathbb{N})$, included in the Mann-Whitney test (table I, appendix), is given for each group. The actual number of displayed legs (each $\operatorname{leg}=$ one dot) is less than indicated in some groups $(0,1,4)$, because legs with a negative TT are omitted. 
occluded SFA with or without aorto-iliac lesions (groups 3 and 5 in fig. 4.8). This finding was similar if the SFA was stenotic although the number of legs with combined stenoses in the aorto-iliac and SFA pathways (group 2) was small. $T_{P D}$ and $T_{C} T_{W}$ are pairwise presented, showing no differences between their value range in all investigated groups.

The pulsed Doppler system enabled the examiner to measure PI at three arterial sites (the common femoral artery, the deep femoral artery and the popliteal artery) in the femoro-popliteal segment from which two meaningful damping factors could be calculated. These damping factors, $\Delta$ com-poppD and $\triangle$ prof-popPD, carry all the information present in the observed differences between the PI's as measured at these three sites. With the CW instrumentation only one damping factor could be calculated, $\Delta$ com-pop $_{C}$. Fig. 4.9 and 4.10

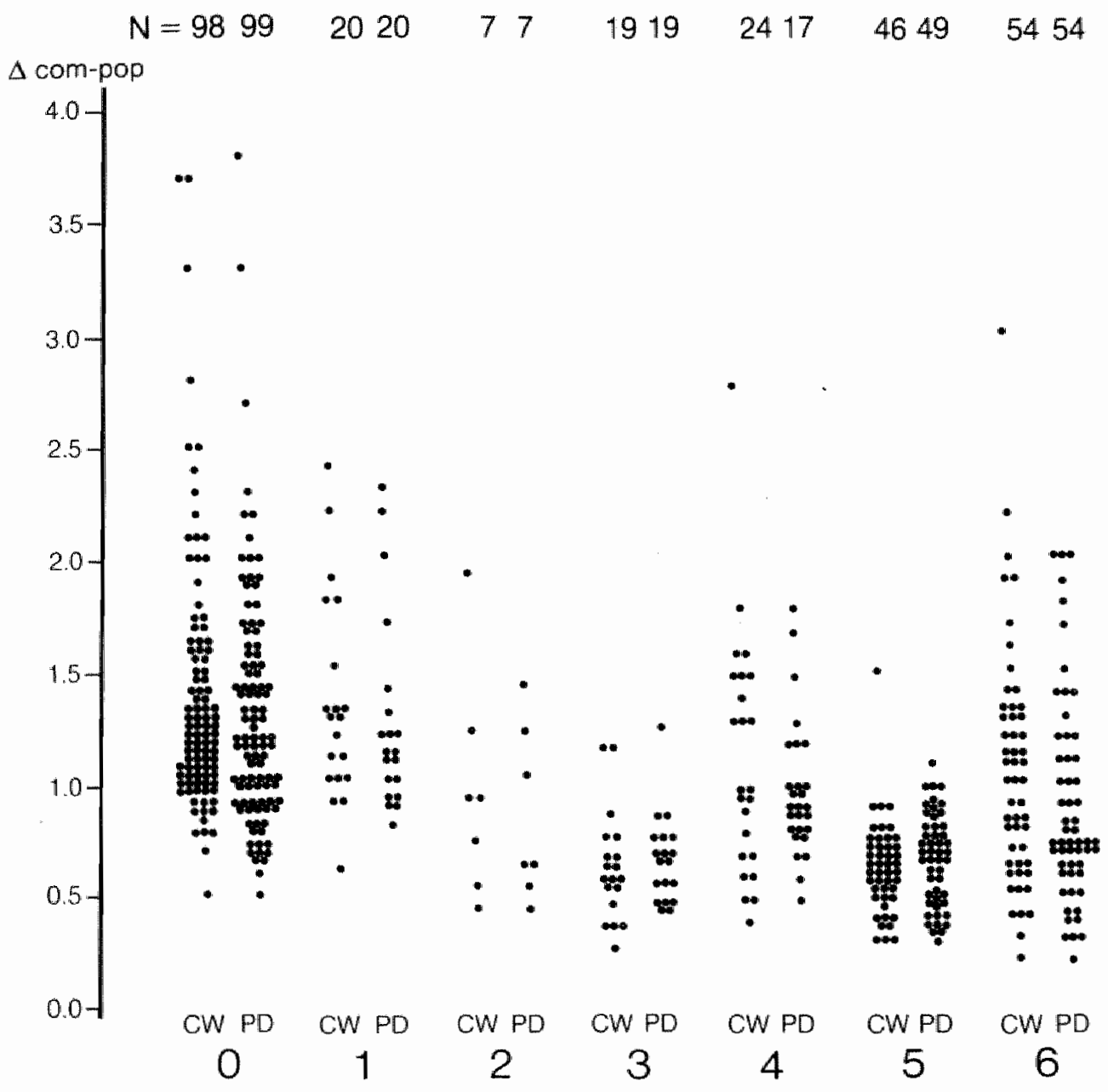

Fig. 4.9 Damping of the velocity wave form between the common femoral artery and the popliteal artery, obtained with both instrumentations, is vertically shown for each leg in all arteriographic groups as denoted in table 4.3. $\Delta$ com-poppD and $\Delta$ com-popcw are pairwise presented for each group and indicated respectively as PD and CW at the foot of each column. The number of legs (N), included in the Mann-Whitney test (table $\mathrm{I}$, appendix), is given. 
show these calculated damping factors for each leg in the six arteriograhic groups as defined in table 4.3. The difference of the mean value of damping between the six groups was statistically evaluated with the Mann-Whitney test (tabel $\mathbb{I}_{\text {, appen- }}$ dix). The value range of the three damping factors within each arteriographic groups is similar. No statistically significant differences are observed between the mean damping value of groups 3 and 5 , groups 2 and 4 , or between groups 0 and 1 so that the conclusion is justified that the calculation of damping in the femoropopliteal pathway is undisturbed by aorto-iliac disease.

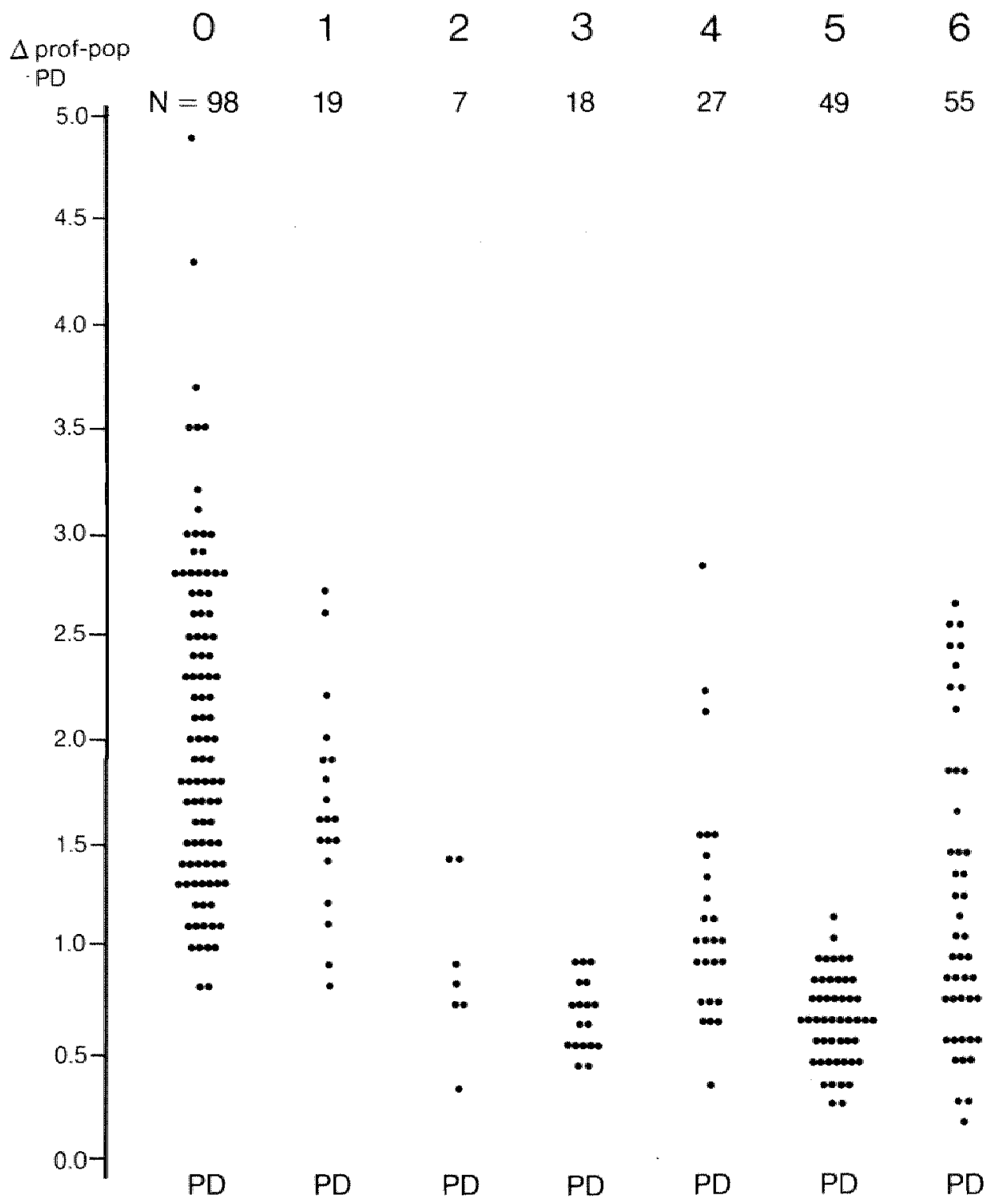

Fig. 4.10 Damping of the velocity wave form between the deep femoral artery and the popliteal artery ( $\Delta$ prof-poppD) is vertically shown for each leg in all arteriographic groups as denoted in table 4.3. The number of legs $(N)$, included in the Mann-Whitney test (table I, appendix), is given. 
The diameter estimation of the SFA with the pulsed Doppler system at 15 and $20 \mathrm{~cm}$ 's below the inguinal ligament is given for each leg in the six arteriographic groups (fig. 4.11). The Mann-Whitney test (table I, appendix) confirms that no statistically significant differences are present between the mean diameter of the SFA at 15 and $20 \mathrm{~cm}$ 's below the inguinal ligament of normal legs and legs with aorto-iliac disease (groups 0 and 1), between legs with an SFA stenosis with or without aorto-iliac disease (groups 2 and 4 ) as well as between legs with an occlusion of the SFA with or without aorto-iliac disease (groups 3 and 5).

\subsubsection{Discussion}

Compliance of the vessel wall is the main determinant of the pulse wave velocity, and hence of $\mathrm{TT}_{\text {foot-to-foot }}$ as well as of $\mathrm{TT}_{\text {peak -to-peak }}$, along arteries. The arteriographic classification is based entirely on the most prominent intraluminal

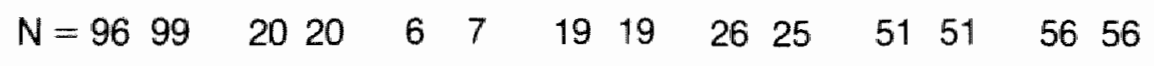

no of chameis

Diam SFA

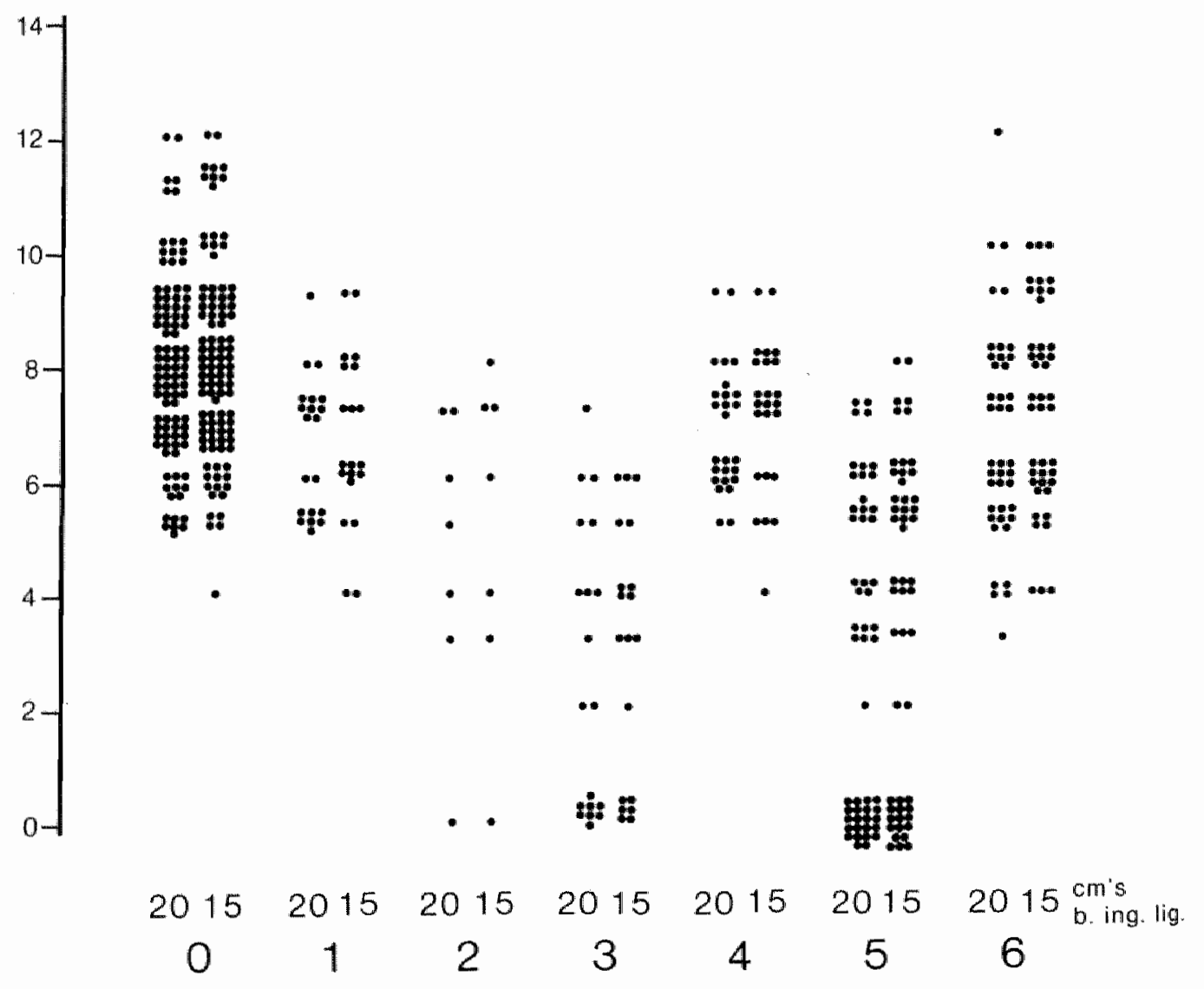

Fig. 4.11 The diameter estimation (number of counted channels) of the superficial femoral artery (SFA) at 15 and 20 cm's below the inguinal ligament, obtained with the multi-gate pulsed Doppler instrumentation, is vertically shown for each leg in all arteriographic groups as denoted in table 4.3. The number of legs $(\mathrm{N}$ ), included in the Mann-Whitney test (table I, appendix), is given. 
changes as seen on the arteriogram, so that neither generalized narrowing of the vessel, differences in the length or efficiency of collateral networks, nor different lengths of occlusion are expressed in this classification. These unexpressed ana-

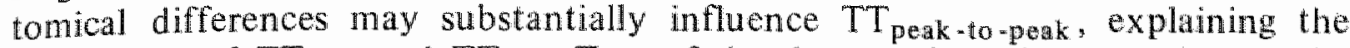
wide range of $T T_{C W}$ and $T T_{P D}$. Two of the three patients in group 3 with the lowest values of TTPD had enormously developed collaterals and wide arterial systems in the presence of a SFA occlusion.

The influence of age, sex and blood pressure on $\mathrm{TT}_{\text {foot-to-foot }}$ has been established in a population free from atherosclerotic symptoms $(64,67,71)$. The eftect of obliterative disease can be expected to be greater than these subtle physiological entities. It is therefore impossible to estimate separately the influence of all causative factors in an individual patient. One would be tempted to explain the slightly increased transit-time (decreased pulse wave velocity) in the femoropopliteal area in patients with aorto-iliac disease (group 1) by the lowered intraluminal pressure distal to the lesion. It could also be argued, referring to the general nature of atherosclerotic disease, that stiffer vessel walls would lead to acceleration of the pressure wave in such cases, thus counteracting the effect of the lower blood pressure (73).

The scatter of damping along its value range in each arteriographic group is similar for the three damping factors, although some difference in the observed overlap seems to be present between $\triangle$ prof-popPD and $\Delta$ com-poppD or $\Delta c o m-p o p_{C W}$ in groups 0 and 5 . The remark made earlier in this section that many anatomical and physiological differences between patients are expressed improperly or not at all in the arteriographic classification, is also valid in relation to damping.

Aorto-iliac lesions are not expected to influence the diameter of arteries relatively far removed from this segment which is confirmed by the absence of a statistically significant difference between groups 3 and 5 , and between groups 2 and 4. The difference in SFA diameter between legs without arterial disease (group 0) and legs with aorto-iliac stenosis (group 1) portrays the generalized nature of atherosclerosis. A second, more daring, explanation of observed but non-significant differences between the legs with and without aorto-iliac lesions speculates that the distal lower-than-normal intra-arterial pressure has a deflatory effect on the arterial lumen.

It has been established in this section that damping and $\mathrm{TT}_{\text {peak-to-peak, }}$, calculated from measurements in the femoro-popliteal pathway are relatively insensitive to the presence of aorto-iliac lesions. The pulsed Doppler system appears to be useful because the overlap between the six arteriographic groups seems less if the damping factor, calculated from Pl in the deep femoral and popliteal arteries, is used. It is furthermore suggested that the ultrasonic diameter estimation of the SFA at 15 or $20 \mathrm{~cm}$ 's below the inguinal ligament is supplying relevant information on the extent of disease in the SFA, the diameter being also independent of arto-iliac lesions. 


\subsection{Is the velocity signal in the common femoral artery influenced by distally located artherosclerotic lesions?}

\subsubsection{Results}

If damping of the velocity wave form such as occurring during its passage through a diseased femoro-popliteal segment, is specific for lesions in this area, it should not be affected by aorto-iliac disease (section 4.5), nor should the proximal signal obtained at the common femoral artery be influenced by more distally located lesions in the femoro-popliteal pathway.

It was the impression during this study, that the contours of the velocity signal from the common femoral artery had changed frequently in the presence of occlusive disease in the femoro-popliteal vessels. The reversed flow component of the normal wave form was replaced by a dicrotic 'notch' on the deceleration side of the wave form in the early diastolic phase, while maintaining a forward direction of flow (fig. 3.11 A). Such a qualitatively defined signal was present in $38 \%$ of the legs with an occluded SFA without arteriographic evidence of significant aorto-iliac disease (table 4.4). None of the normal legs and none of the legs with aorto-iliac disease demonstrated this particular wave form so that the change in wave form seems to be related to the presence of occlusive disease in the SFA.

Signals with a dicrotic "notch' on the deceleration side of the systolic peak have an additional feature which is easier to quantify (fig. 3.11 A). The backflow component of the normal wave form seems to be replaced by the dicrotic "notch", leaving the diastolic phase of the wave form relatively unchanged. When severe aorto-iliac disease is present, forward flow velocities are maintained during diastole as a result of the greatly lowered peripheral impedance. Consequent to these described changes in the wave form, one may distinguish signals with an equation

Table 4.4 The velocity wave form obtained with the pulsed Doppler system in the common femoral artery, classified as 'normal" (figure $3.11 \mathrm{~A}$ right leg), dicrotic 'notch' (figure $3.11 \mathrm{~A}$, left leg) or 'diastolic forward velocities' (figure 3.10B). This classification was given to the common femoral artery in eight arteriographic groups. The frequency of a particular wave form is given as a percentage of the total number of legs available for qualitative judgment in each arteriographic group. $\mathrm{AI}=$ aorto-iliac; proximal $\mathrm{SF} A=$ from the bifurcation of the common femoral artery to approximately 10 cm's above Hunter's canal; distal SFA = SFA from $10 \mathrm{~cm}$ 's above Hunter"s canal to the distal popliteal artery.

\begin{tabular}{lcccc}
\hline & Normal & Dicrotic 'notch' & $\begin{array}{c}\text { Diastolic forward } \\
\text { velocities } \\
\%\end{array}$ & No.of legs \\
Normal & $\%$ & $\%$ & 1 & 97 \\
Al stenosis & 99 & 0 & 50 & 14 \\
Al occlusion & 50 & 0 & 90 & 10 \\
Al stenosis, SFA stenosis & 10 & 0 & 50 & 8 \\
Al stenosis, SFA occlusion & 8 & 37.5 & 50 & 18 \\
SFA stenosis & 87.5 & 12.5 & 0 & 24 \\
Proximal SFA occlusion & 35 & 38 & 27 & 26 \\
Distal SFA occlusion & 40 & 26 & 32 & 25 \\
\hline
\end{tabular}


$V_{\text {peak-to-peak }} / V_{\text {max. systole }}>1$ (normal), equal to 1 (dicrotic "notch') and $<1$ (severe aorto-iliac disease). These calculations were carried out on the velocity signals obtained with both instrumentations and the results summarized in table 4.5. The wave form with the equation $V_{\text {peak-to-peak }} / V_{\max }$ systole equals 1 appeared to occur most frequently in legs with an occlusion of the SFA. The summarized results in the tables 4.4 and 4.5 are not strictly comparable because many signals in patients with SFA and/or aorto-iliac disease, show absence of back flow without evidence of a dicrotic 'notch'.

The high percentage of common femoral artery signals showing absent back flow in the analog recording, when significant atherosclerotic disease is restricted to the SFA, implies that the velocity signal can be influenced by more distally located lesions. However, a possibility remains that atherosclerotic changes of the aorto-iliac arteries, undoubtedly present in most patients with symptomatic arterial disease, whether visible on the arteriogram or not, are responsible for this decrease of pulsatility. A small group of seven patients with unilateral disease in the SFA without arteriographic signs of significant aorto-iliac lesions was therefore evaluated. The contralateral SFA of these seven patients was considered free from significant atherosclerotic disease because the ankle pressures on that side were normal. It is shown (fig. 4.12 ) that the equation $\mathrm{V}_{\text {peak-to-peak }} / \mathrm{V}_{\max }$ systole is considerably lower on the side with SFA disease.

Table 4.5 The ratio $\mathrm{V}_{\text {peak-to-peak }} / \mathrm{V}_{\max }$ systole was calculated from the velocity wave form obtained in the common femoral artery with both instrumentations (PD and CW) in eight arteriographic groups. The ratio can be $>1=$ normal wave form, equal to $1=$ no backllow or $<1=$ diastolic forward velocities as frequently observed with severe AI disease. The frequency of a particular ratio is given as a percentage of the total number of legs available for this type of analysis in each arteriographic group.

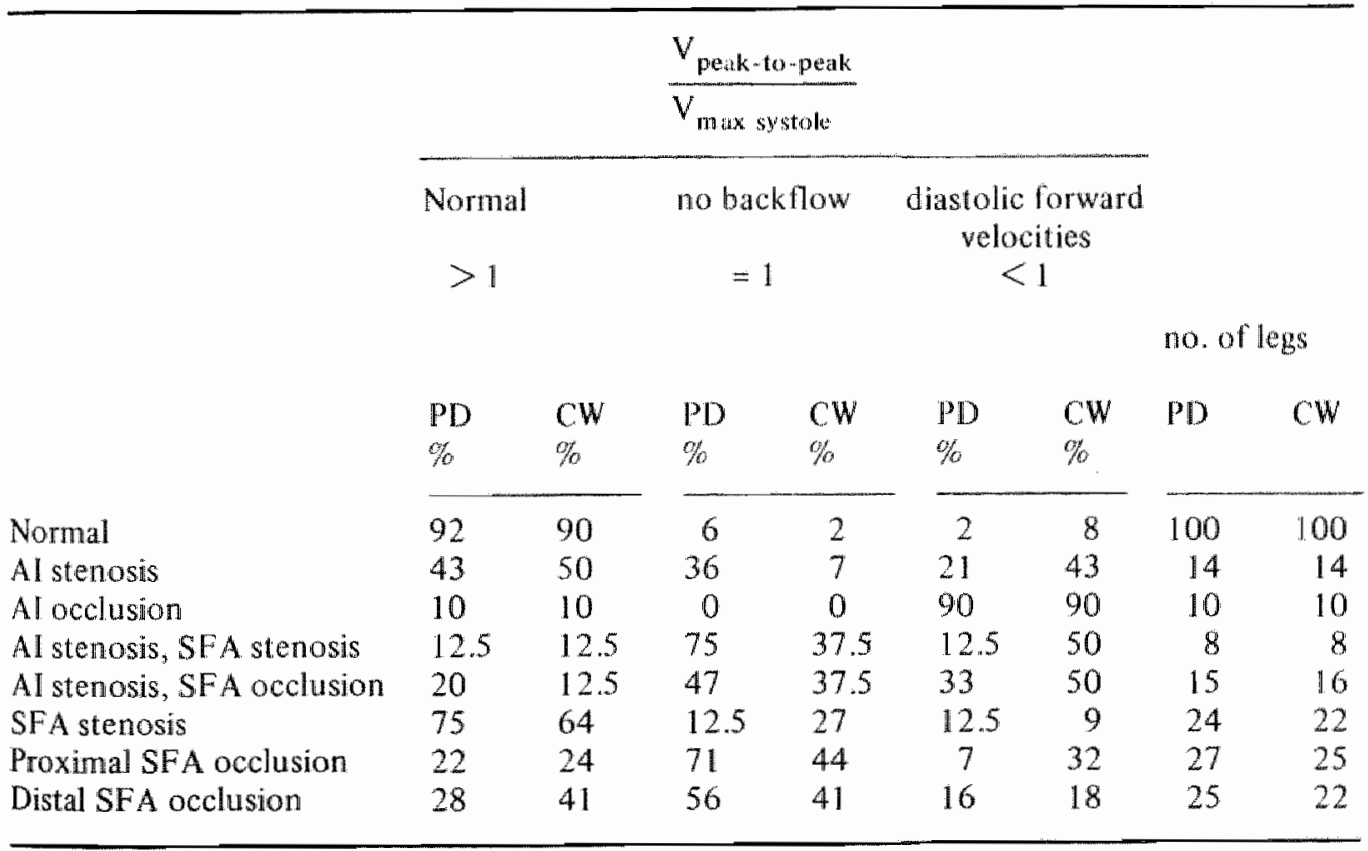




\subsubsection{Discussion}

The presented data strongly suggest that atherosclerotic disease distal to the common femoral artery announces its presence through changes in the velocity wave form recorded in this artery. Thirty-eight percent of the legs with an occlusion in the proximal part of the SFA showed this so-called dicrotic "notch" while $71 \%$ appeared to show no signs of back flow or diastolic forward flow in the analog tracing of the common femoral artery as obtained with the pulsed Doppler instrument.

Busse (33) stated that the described alterations of the velocity wave form result from generalized degenerative changes in the aorto-iliac arterial system. In 14 legs with an aorto-iliac stenosis none of the cases displayed this dicrotic 'notch' (table 4.4), and in a group of seven patients with unilateral SFA disease, without arteriographic evidence of aorto-iliac abnormalities, wave form distortions were seen predominantly on the side of the SFA lesion. These findings indicate that aortoiliac degenerative disease is not likely to be responsible for velocity wave form changes described as dicrotic 'notch'.

The pressure and flow pulse can be regarded as a summation of forward and backward (or reflected) waves $(33,159)$. Forward waves run into the system following the myocardial contraction, while backward waves are the result of reflections of forward waves bouncing from arterial sites where impedance mismatch is present $(33,102,111,159)$. Under ideal conditions, the pressure wave is transmitted down the arterial tree with a minimum of reflections at large bifurcations $(65,89,102)$, the arteriolar part of the arterial tree being the predominant site of strong but variable reflections $(102,117)$. 'Impeding' atherosclerotic

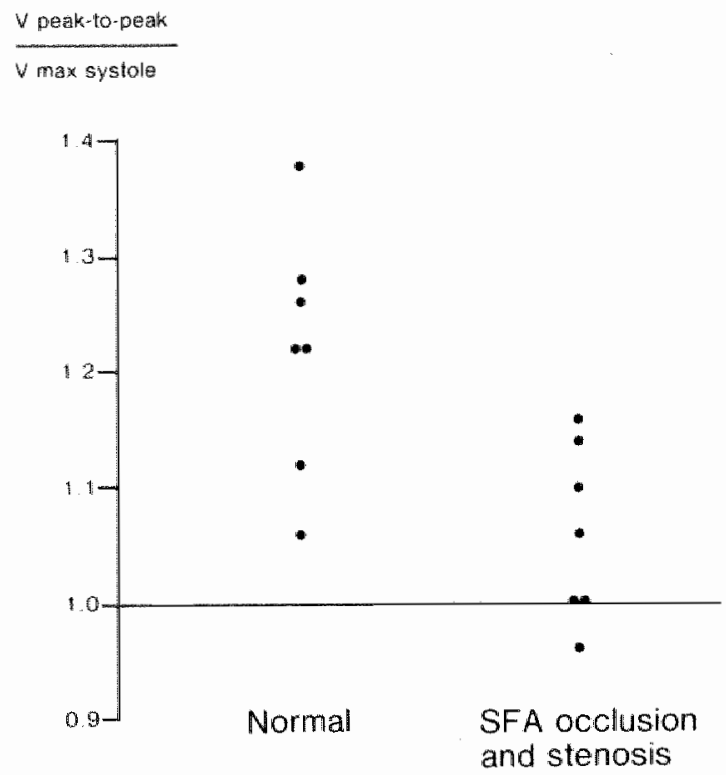

Fig. 4.12 The velocity wave form, obtained with the multi-gate pulsed Doppler instrumentation in both common femoral arteries of seven patients with unilateral disease of the superficial femoral antery (SFA), are compared. The ratio $V_{\text {peak-to-peak }} / V_{\text {max systole }}$ (vertical) is given for the normall legs (ASPI $\geqslant 95 \%$ ) and the legs with an SFA occlusion or stenosis. 
lesions may thus be expected to result in impedance changes, causing the reflection of pressure waves.

Experimental evidence $(111,159)$, strenghtened by clinical observations in the carotid artery (13) and the femoral artery (112), supports the view that changes in the Doppler wave form contour, proximal to atherosclerotic lesions are caused by reflections of pressure waves at the site of disease. Reflected pressure waves give rise to node and anti-node formation of pressure and flow only in the immediate vicinity of the reflection site, because of the rapid dissipation of the reflected energy at greater distances. It was stated by Newman (111), that assessment of a vascular obstruction, by means of a proximal measurement, must be carried out close to the point of reflection. This statement can be appropriately rephrased by saying that PI measurements should be carried out as far away as possible from a site of reflection to diminish its influence on the shape of the velocity wave form. The site and geometrical properties of an atherosclerotic lesion in the SFA is different in each patient, so that its influence on the proximal velocity signal will vary accordingly. This may explain why the dicrotic 'notch' is not consistently found in all legs with an occluded SFA.

The described change of the wave form contour has a profound influence on the calculated damping factor between the common femoral and the popliteal arteries following the decrease of peak-to-peak and mean velocity values of the wave form, and subsequently, on the computed PI. This may explain some of the overlap of damping between the arteriographic groups (section 4.5).

Reflected pressure waves from somewhere in the SFA may have a greater effect on the velocity wave form in the common femoral artery than on the signal in the deep femoral artery. This is supported to some extent by the difference between the shape of the wave form of four deeper situated channels of the common femoral artery, as compared to six channels 'looking' at the SFA (fig. 3.11A). The diminished influence of reflections on the wave form of the deep femoral artery may possibly explain why the observed overlap between the range of $\Delta$ prof-poppD of the arteriographic groups described in section 4.5 is less than between $\Delta$ com-poppD. The velocity peak in the superficially situated channels did not appear to be displaced to a notable degree which was the reason that $\mathrm{TT}_{\text {peak }}$. to-peak in relation to distal or proximal SFA disease was not examined.

\subsection{The hemodynamic parameters in relation to femoro-popliteal disease}

\subsubsection{Results}

This section describes the relationship between the three parameters - damping, $\mathrm{TT}_{\text {peak -to-peak }}$ and arterial diameter estimation - and atherosclerotic lesions of different extent and severity in the femoro-popliteal pathway.

Section 4.5 established that aorto-iliac disease has no influence on damping and

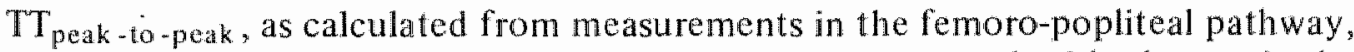
so that observed changes in the parameter values correspond with changes in the hemodynamic properties of the vessel segment between the sites of measurement in the common femoral and popliteal arteries. The variable nature of lesions in this area with regard to their location and effect on the ankle pressure(s) has already been pointed out in the sections 3.3 (fig. 3.1) and 4.3 (fig. 4.7) for which reason it was considered essential to evaluate these parameters in relation to 
various combinations of atherosclerotic lesions in the three arterial segments of interest (proximal and distal segment of the SFA, proximal part of the popliteal artery).

This resulted in the formation of 21 arteriographic subgroups (a-u), each with a different pattern of atherosclerotic disease. Separate evaluation of each of the 21 arteriographic subgroups was considered to be statistically unsound because of the small number of legs in many of these subgroups. The legs under study were therefore re-arranged into three larger groups $(0=$ normal, $1=$ stenosis, $2=$ occlusion). If one or more stenoses were present in addition to an occlusion, the leg was assigned to the group 'occlusions', no matter their localization (table 4.6).

Fig. 4.13 to 4.19 show the value of each hemodynamic parameter per leg over the 21 arteriographic subgroups (legs in box), their re-allocation into groups 0,1 and 2 is indicated with an arrow. The statistical differences between the mean of these groups $(0,1,2)$ are highly significant for all parameters except for the diameter estimations (results of Mann-Whitney tests, table II in appendix).

\subsubsection{Discussion}

All figures (fig. 4.13 to 4.19 ) have similar features regarding the degree of overlap of the parameter values between the arteriographic groups 0,1 and 2 . None of the

Table 4.6 Twenty-one arteriographic subgroups (a-u) are formed using the arteriographic information of the arterial segments of interest (proximal and distal SFA, proximal popliteal artery). The rearrangement into three larger groups $(0=$ normal, $1=$ stenosis, $2=0$ cclusion $)$ is shown.

SUBGROUP

No. of legs

a normal

normal group

(128) 0

128

b stenosis prox. SFA

c stenosis dist. SFA

d stenoses prox. and dist. SFA

e stenosis popliteal artery

$r$ sitenoses prox. SFA + pop. art.

g stenoses dist. SF $\mathrm{A}+$ pop. art.

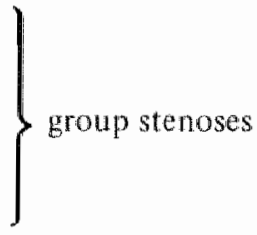

Ih occlusion prox. SFA

occlusion dist. SFA

stenosis prox. SFA + occlusion dist. SFA

$k$ occlusion prox. SFA + stenosis dist. SFA

I occlusion prox. SFA + occlusion dist. SFA

occlusion dist. SFA + stenosis poplitea

$n$ sten. prox. SFA + ocel. dist. SFA + sten, pop.

o ocel. prox. SFA + sten. dist. SFA + sten. pop.

p ocel. prox. SFA + occl dist. SFA + sten. pop.

$q$ occlusion poplitea

r stenosis dist. SFA + occlusion poplitea

$\$$ occlusion dist. SFA + occlusion poplitea

$t$ sten. prox. SFA + ocel, dist. SFA + ocd. pop.

u ocel prox. SFA + ocel dist. SFA + ocd. pop.

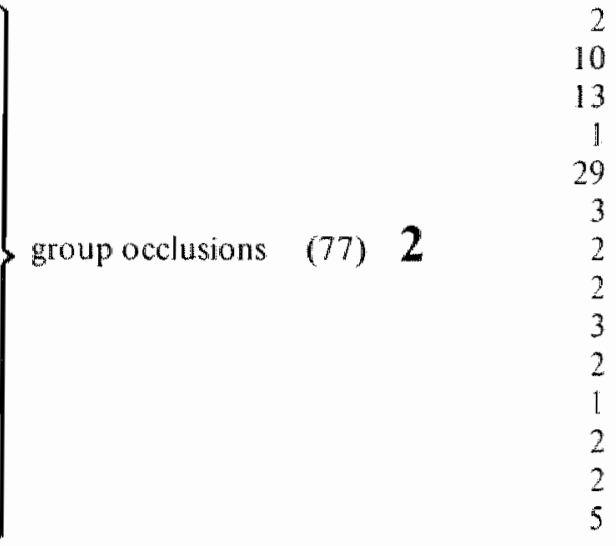




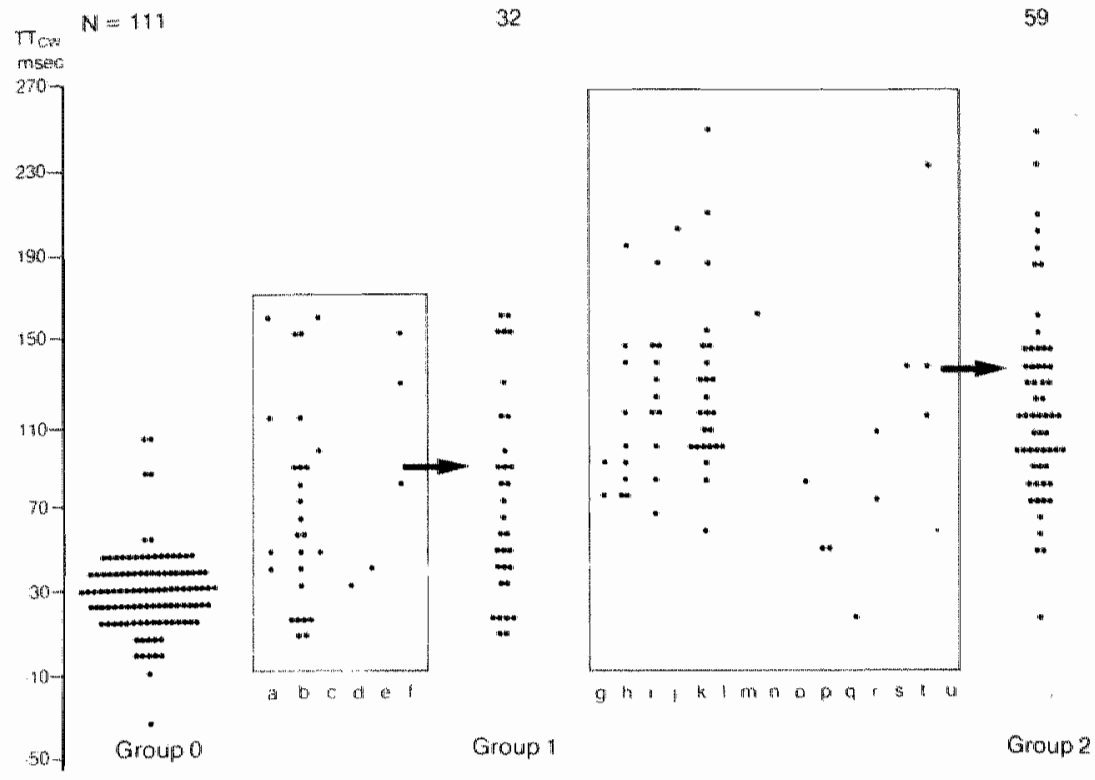

Fig. 4.13 The transit-time ( $\mathrm{TT}_{\text {peak-to-peak }}$ in msec) of the velocity wave between the common femoral artery and the popliteal artery, obtained with the $\mathrm{CW}$ instrumentation ( $\left.\mathrm{TT}_{\mathrm{CW}}\right)$, is given over 21 arteriographic subgroups (a-u, table 4.6). Their rearrangement into larger groups $(1,2)$ is indicated with an arrow. $\mathrm{N}=$ number of legs in each group $(0,1,2)$.

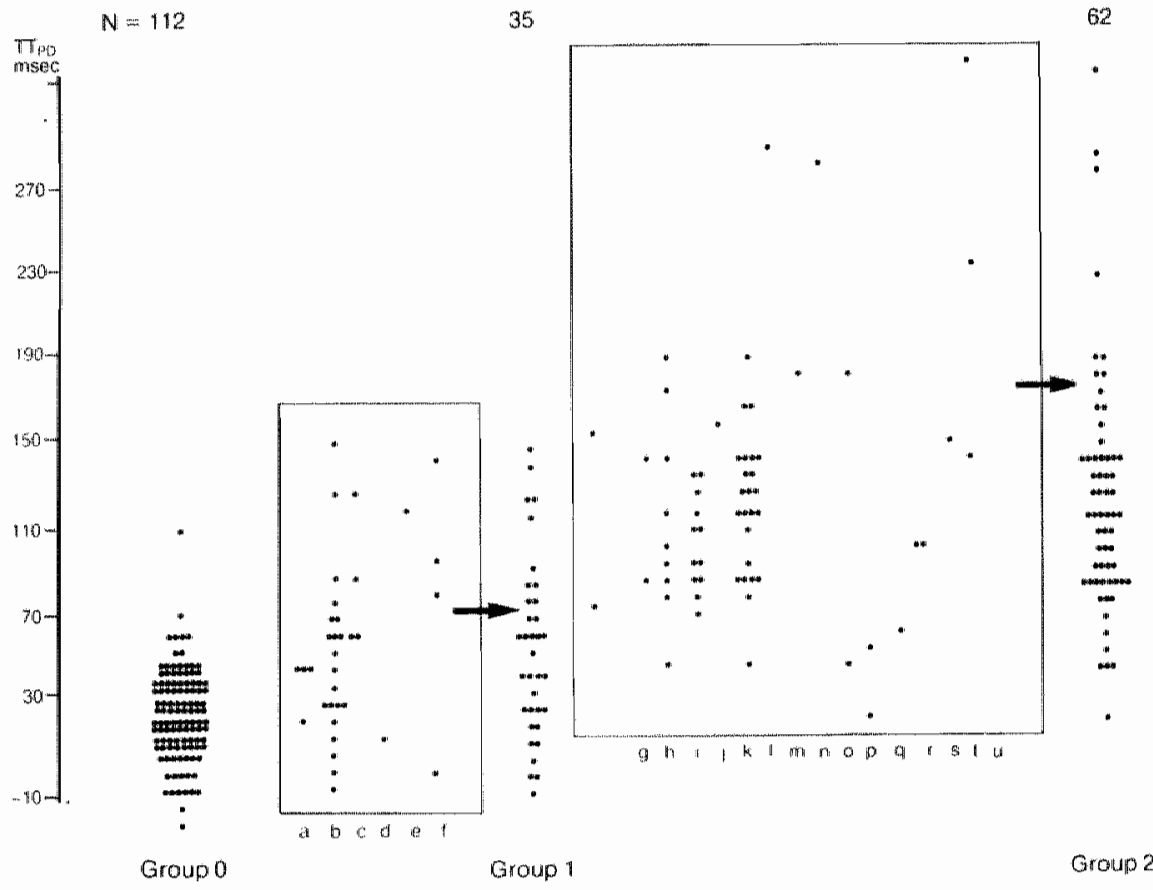

Fig. 4.14 The transit-time ( $\mathrm{TT}_{\text {peak-to-peak }}$ in msec) of the centerstream velocity wave between the common femoral artery and the popliteal artery, obtained with the multi-gate pulsed Doppler instrumentation (TTPD), is given over 21 arteriographic subgroups (a-u, table 4.6). Their rearrangement into larger groups $(1,2)$ is indicated with an arrow. $N=$ number of legs in each group $(0,1,2)$. 


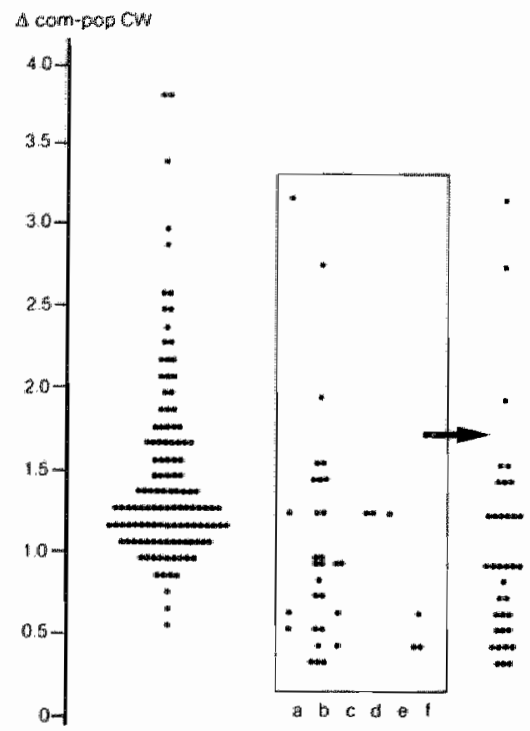

Groupo

Giroup 1

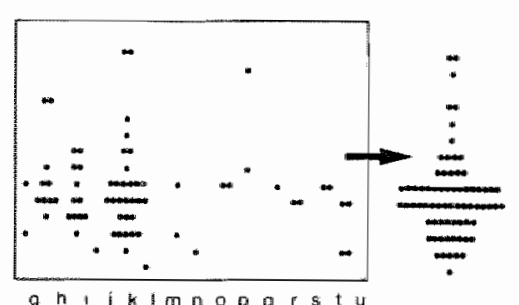

Group 2

Fig. 4.15 Damping of the velocity wave between the common femoral artery and the popliteal artery as determined with the $C W$ instrumentation $(\Delta$ com-pop $\mathrm{cw})$ is given over 21 arteriographic subgroups (a-u, table 4.6). Their rearrangement into larger groups (1.2) is indicated with an arrow. $\mathrm{N}=$ number of legs in each group $(0,1,2)$.

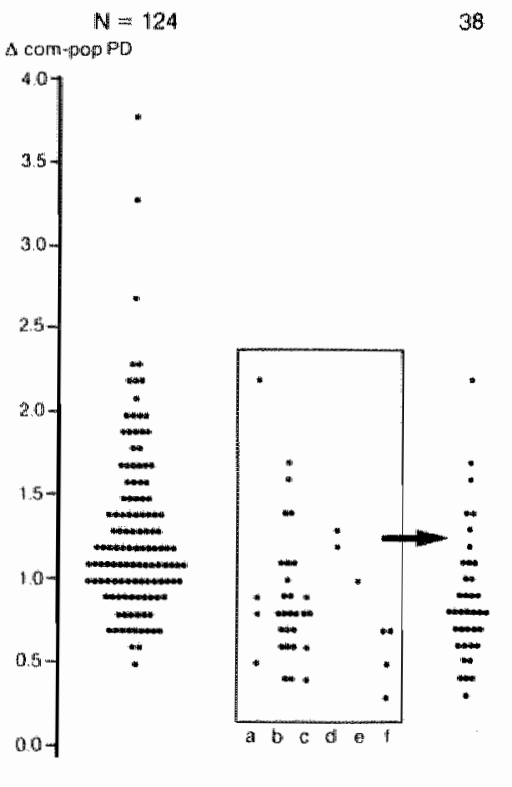

Group 0

Group 1

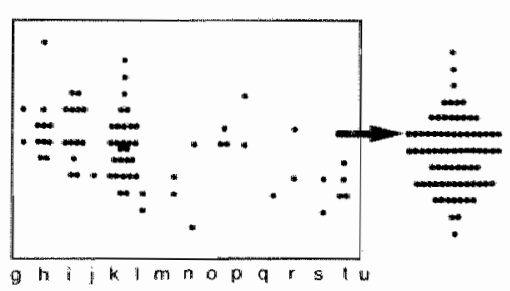

Group 2

Fig. 4.16 Damping of the centerstream velocity wave between the common femoral artery and the popliteal artery as determined with the multi-gate pulsed Doppler instrumentation $(\Delta$ com-poppD), is given over 21 arteriographic subgroups (a-u, table 4.6$)$. Their rearrangement into larger groups $(1.2)$ is indicated with an arrow. $N=$ number of legs in each group $(0,1,2)$. 


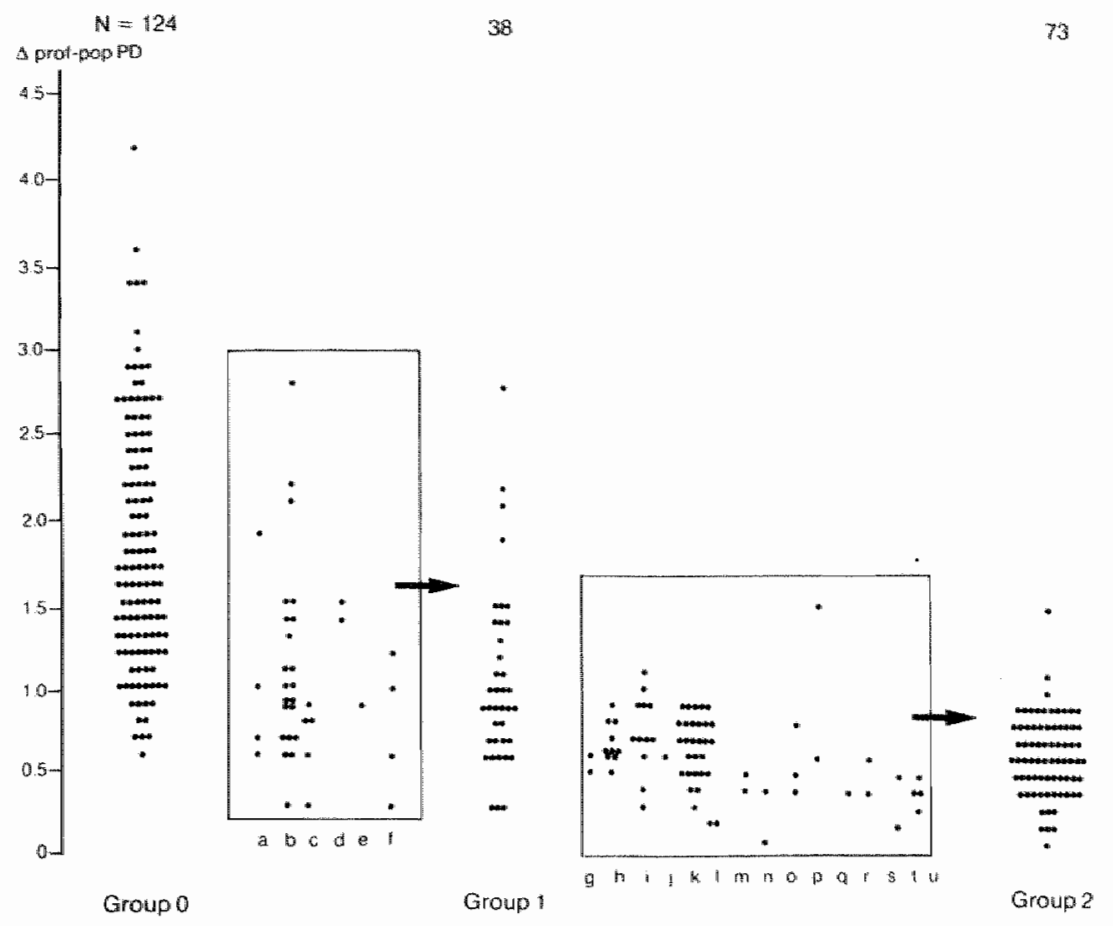

Fig. 4.17 Damping of the centerstream velocity wave between the deep femoral artery and the popliteal artery as determined with the multi-gate pulsed Doppler instrumentation ( $\Delta$ profpopPD), given over 21 arteriographic subgroups (a-u, table 4.6). Their rearrangement into larger groups (1.2) is indicated with an arrow. $N=$ number of legs in each group $(0,1,2)$.

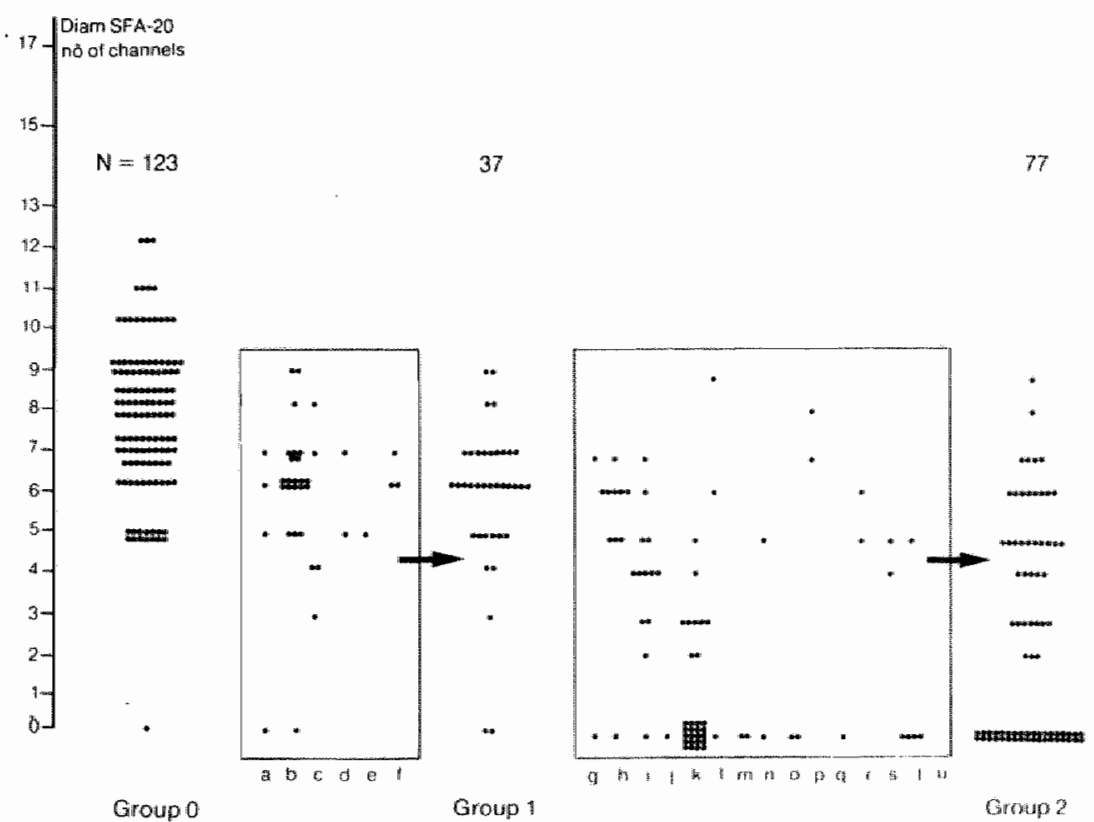

Fig. 4.18 The diameter of the superficial femoral artery (Diam SFA-20) at $20 \mathrm{~cm}$ 's below the inguinal ligament estimated with the multi-gate pulsed Doppler system, is given over 21 arteriographic subgroups ( $a-u$, table 4.6$)$. Their rearrangement into larger groups $(1,2)$ is indicated with an arrow. $N=$ number of legs in each group $(0,1,2)$. 


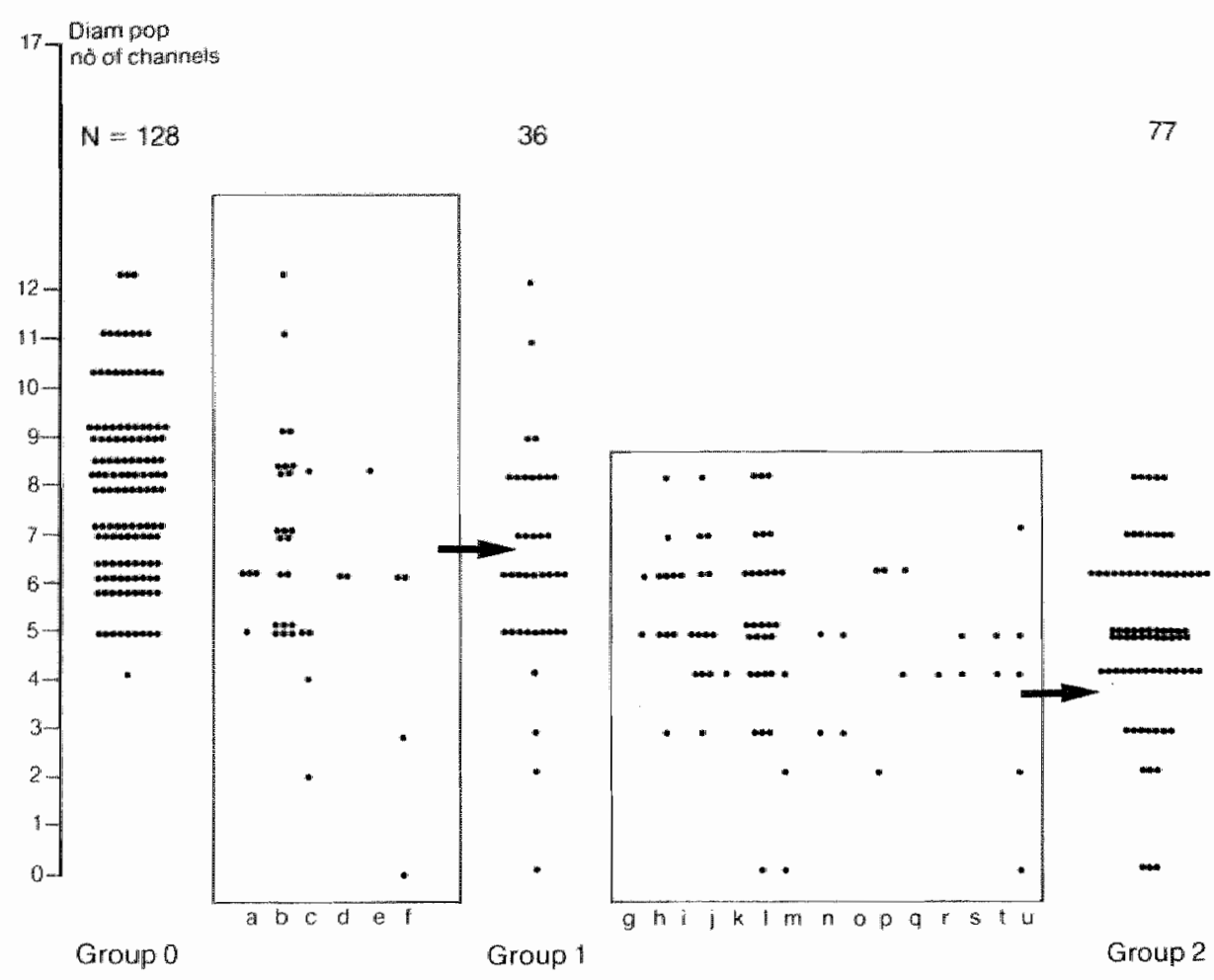

Fig. 4.19 The diameter of the popliteal artery $\left(\right.$ Diam $\left._{\text {pop }}\right)$ estimated with the multi-gate pulsed Doppler system, is given over 21 arteriographic subgroups (a-u, table 4.6). Their rearrangement into larger groups $(1,2)$ is indicated with an arrow. $N=$ number of legs in each group $(0,1,2)$.

parameters can individually distinguish between them because of mutual overlap, and no essential differences are noted to exist between both instrumentations. The selected method of presentation emphasizes the heterogenous composition of the group with stenosis (group 1) and the group with at least one occlusion (group 2), regarding their presented arteriographic findings. Although a gross correspondence may be observed between the severity and extent of the lesions in the three arterial segments under consideration and the value of each parameter (compare for example subgroup $u$ with subgroup $q$ ) large differences of damping and TT can also be noted to exist within arteriographic subgroups. Some of this may be the result of the many possible sources of error and inaccuracy as described in section 3.7, but subtle differences regarding the extent of the disease at non-occluded arterial sites, in addition to differences of the collateral response, will also play an important part. The presence of an occlusion somewhere in the femoro-popliteal pathway seems to be associated with a general narrower SFA at $20 \mathrm{~cm}$ 's below the inguinal ligament (fig. 4.6A and B, 4.18). The data systematically displayed in fig. 4.13 to 4.19 will be used for further analysis in section 4.9 . 


\subsection{The effect of age and blood pressure on $T_{\text {peak-to-peak }}$ and damping}

\subsubsection{Results}

The pulse wave velocity through an artery is related to the compliance of the arterial wall and hence to age and intra-luminal blood pressure (section 2.6). Gosling $(64,69)$ showed that correction for age and blood pressure of $\mathrm{TT}_{\text {foot }}$. to-foot contributed to improved noninvasive identification of stenotic or occluded vessel segments when compared with the arteriographic findings.

The effect of age and systolic blood pressure on $\mathrm{TT}_{\text {peak-to-peak }}$ and damping in the femoro-popliteal pathway, was evaluated in 97 legs with a normal ASPI before and after excercise. The age of the patients varied from 30 to 80 years. The Pearson correlation coefficient $(R)$ and the direction coefficients as obtained with linear regression analysis, facilitate the interpretation of the findings.

Neither age nor blood pressure were found to correlate with damping, as is shown by the horizontal course of the regression lines (fig. 4.20). This implies that correction for age (or systolic blood pressure) of this parameter will not increase its ability to discriminate between the three arteriographic groups $(0,1$ and 2$)$ which were described in section 4.7 . TT, on the other hand, decreased $7.5 \mathrm{msec}$ over the full age range of 50 years or over the pressure range of $120 \mathrm{mmHg}$ (fig. 4.21 ). It is doubtful, however, whether the regression lines of $\mathrm{TT}_{\mathrm{PD}}$ on $\mathrm{TT}_{\mathrm{CW}}$ may be interpreted in view of the observed low degree of correlation.

It is obvious that the effect on TT, following correction for age or blood pressure, will be lost in the error or variation of $\mathrm{TT}_{\text {peak -to-peak }}$ measurements as described in sections 3.7 and 4.2 .

\subsubsection{Discussion}

Normalization of damping for age or blood pressure, as determined with both Doppler systems, does not improve its discriminative value for lesions in the femoro-popliteal pathway. Gosling (69) determined the average value of damping in three different arterial pathways in a group of 8 normal subjects with their age ranging from 21-56 years. The damping observed in a particular vascular segment of a patient was divided by the average damping factor of the same vascular segment as found in this group of normal subjects. This 'normalization' procedure was not meant to correct for age, but to adapt the observed damping from different vascular segments (aorto-iliac, femoro-popliteal, popliteo-tibial) for graphic display in the same figure. The findings of Humphries (77) that no differences are present between unmodified and 'normalized' damping factors regarding their discriminative value for lesions in the femoro-popliteal segment is therefore not surprising!

Aging coincides with higher systolic blood pressure (fig. 4.22). It is probable that the decrease of TT observed in patients with increasing age, is largely due to the effect of raised systolic blood pressure which is in accordance with the evidence presented by Gosling (64).

Figure 4.23 compares TTPD/systolic blood pressure (present study) with $\mathrm{TT}_{\text {foot-to-foot }} /$ mean arterial pressure in a group of normal subjects (50-60 years of age) as measured by Gosling in an arterial segment of $40 \mathrm{~cm}$ 's (taken from 

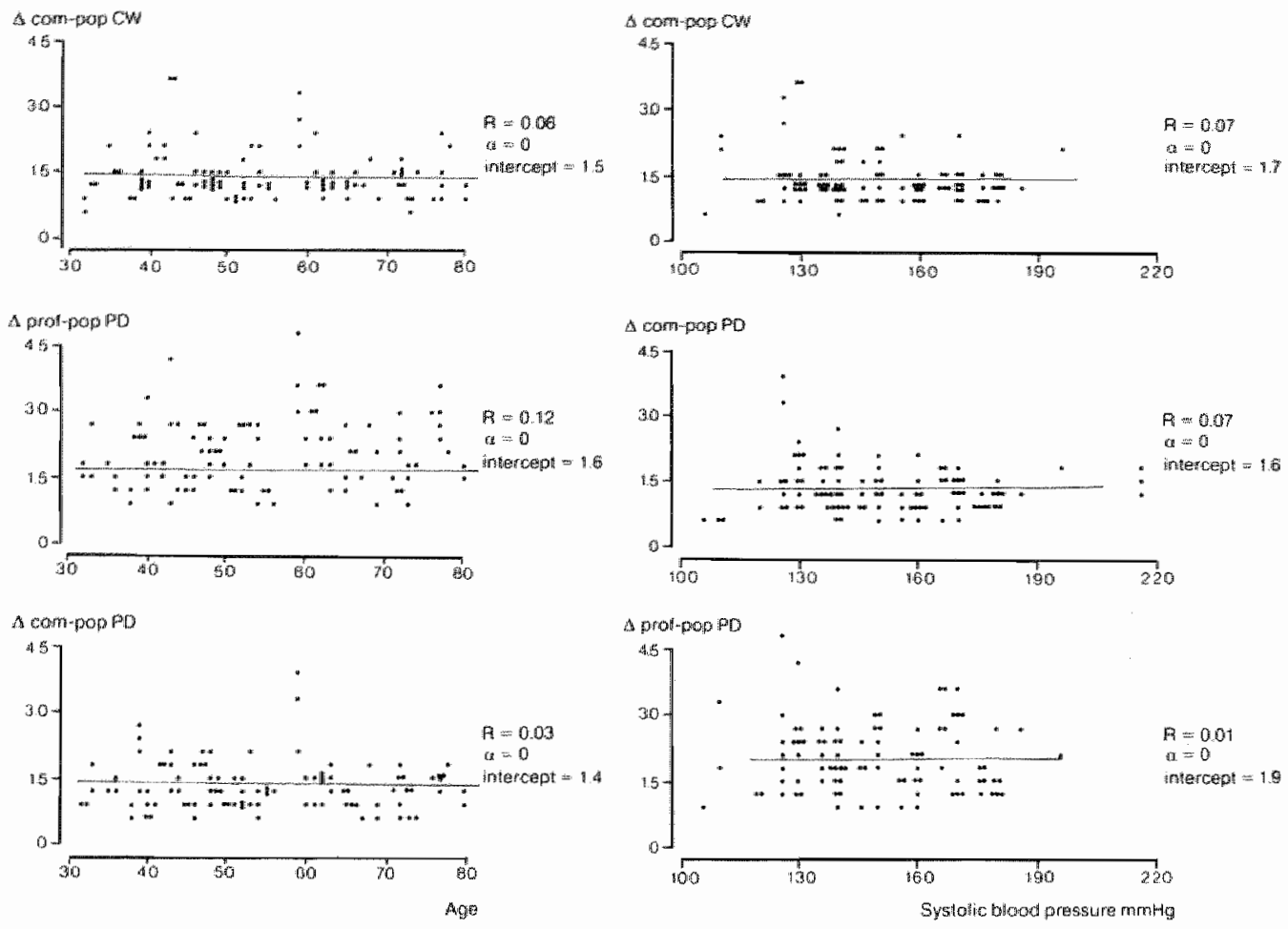

Fig. 4.20 Damping, determined between the common femoral artery and the popliteal artery with both instrumentations ( $\Delta$ com-popcw, and $\Delta$ com-poppD $)$ and damping between the deep femoral artery and the popliteal artery determined with the multi-gate pulsed Doppler system ( $\Delta$ prof-poppo), are related to age ( $30-80$ years) and systolic blood pressure (brachial artery, range $100-200 \mathrm{~mm} \mathrm{Hg}$ ) in 97 legs with normal ankle pressures (ASPI at rest and after exercise $\geqslant$ 95\%). The Pearson correlation coefficient $(\mathbb{R})$, the direction coefficient $(\alpha)$ and the intercept of the regression line are given for each correlation.
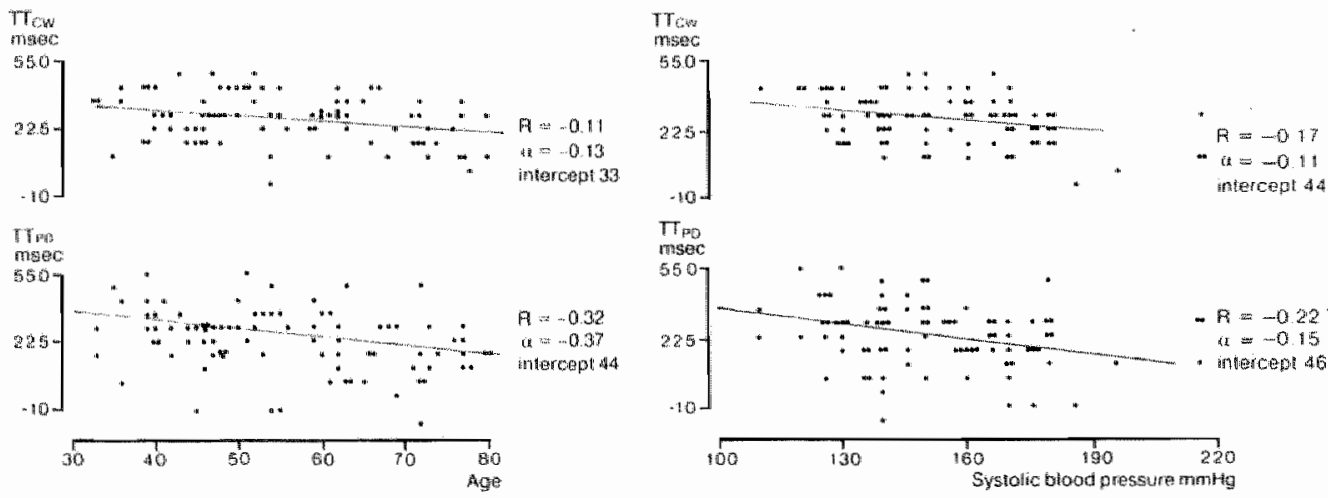

Fig. 4.21 The transit-time of the velocity wave between the common femoral artery and the popliteal artery, obtained with both Doppler instrumentations ( $T T_{P D}$ and $\mathrm{TT}_{\mathrm{CW}}$ ), are related to age (30-80 years) and systolic blood pressure (brachial artery, range $100-220 \mathrm{~mm} \mathrm{Hg}$ ) in 97 legs with normal ankle pressures. (ASPI at rest and after exercise $\geqslant 95 \%$ ). The Pearson correlation coefficient $(R)$, the direction coefficient $(\alpha)$ and the intercept of the regression lines are given for each correlation. 


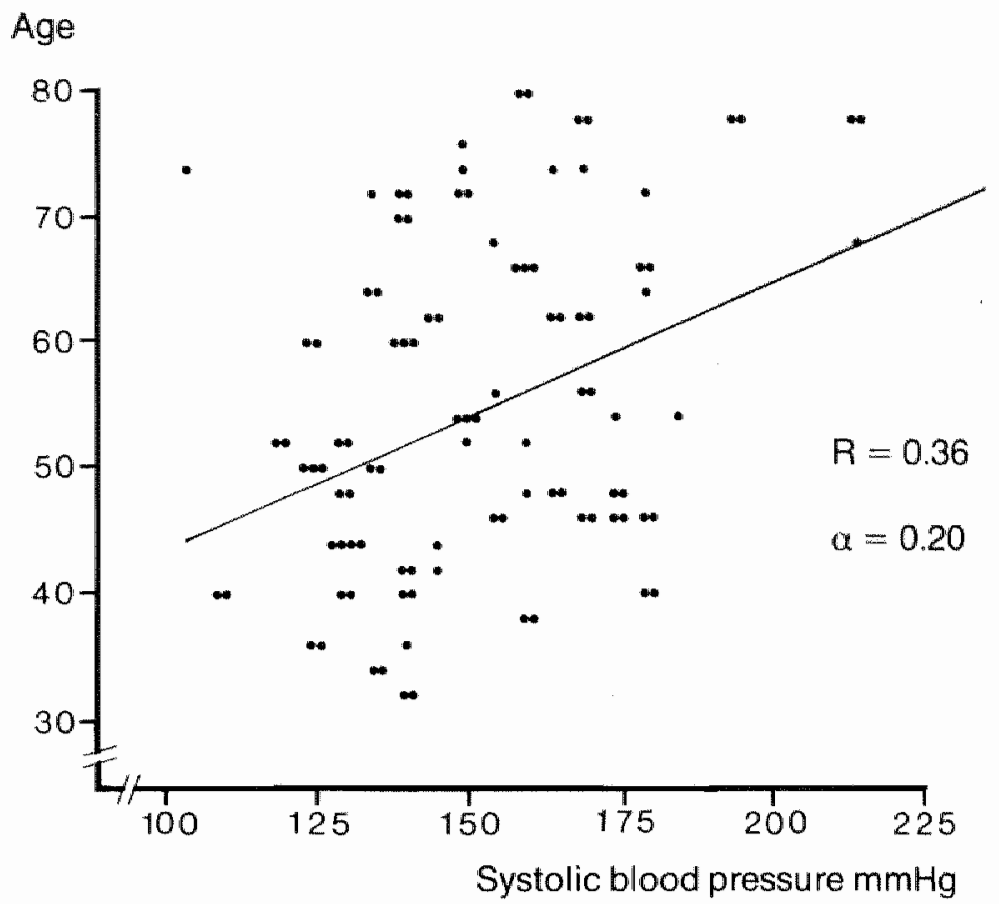

Fig. 4.22 Age and the systolic blood pressure (brachial artery) are correlated in a group of patients without significant arterial disease (ASPI at rest and after exercise $\geqslant 95 \%$ ). $R=$ Pearson coefficient of correlation; $\alpha=$ the direction coefficient of the regression line.

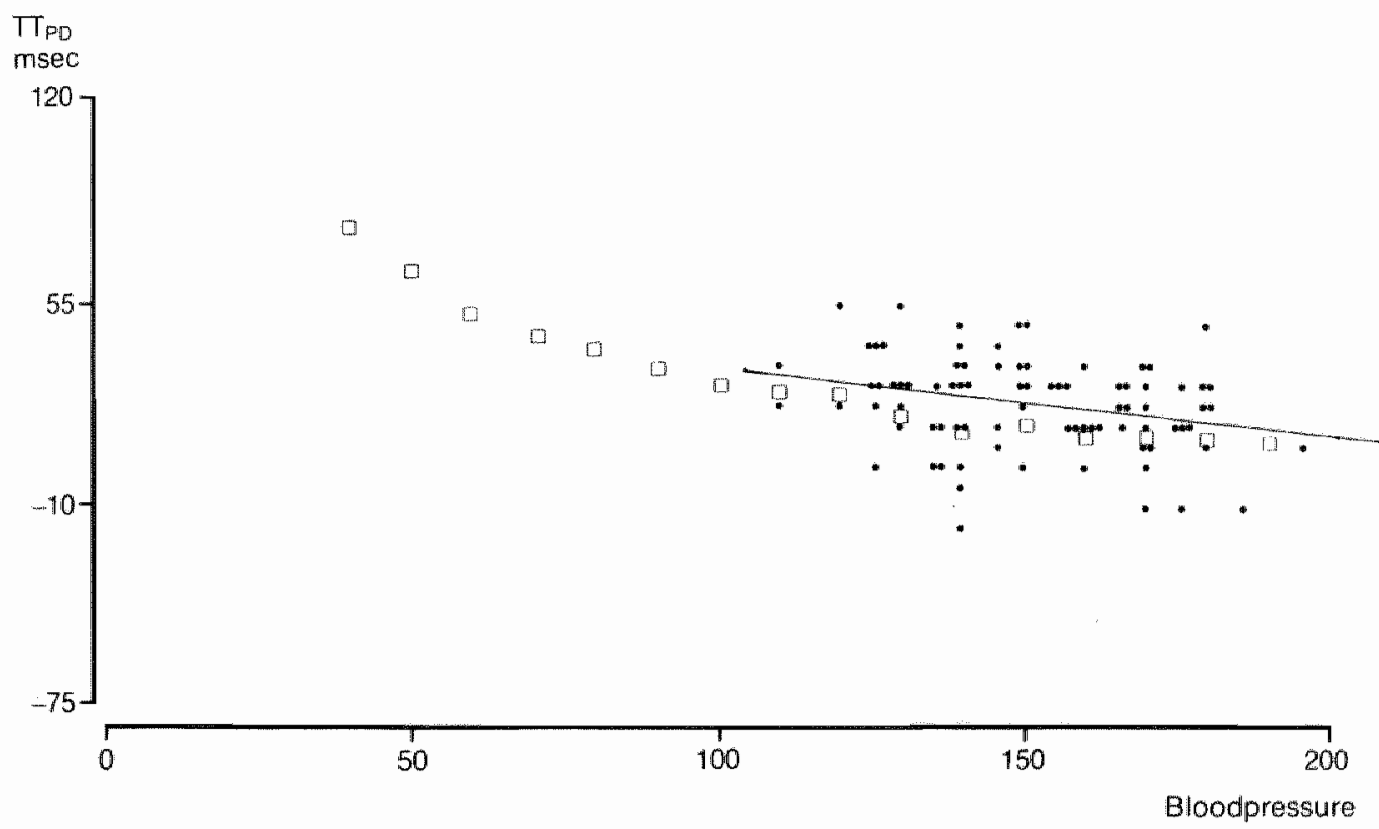

Fig. 4.23 Transit-time peak-to-peak (TTPD) as determined in the femoro-popliteal segment of 97 legs with normall ankle pressures (ASPI at rest and after exercise $\geqslant 95 \%$ ) is compared with transit-time foot-to-foot as measured by Gosling (69) in a vascular segment of $40 \mathrm{~cm}$ 's. TT is vertically given in msec. $\mathrm{TT}_{\text {foot-to-f(x)t }}(\mathrm{a})$ is compared with the mean blood pressure and $\left.\mathrm{TT}_{\mathrm{BD}}\right)$ (•) with the systolic blood pressure (brachial artery) both horizontally displayed. 
Gosling, 69). The regression line (present study) indicates close agreement with the findings of Gosling, showing the accelerating effect on the pulse wave velocity (decrease of TT) with higher arterial pressures.

The "correcting" effect of normalization is maximal at low pressures in the femoro-popliteal area in view of the non-linear relation of TT with pressure. The accuracy of noninvasive pressure measurements in the femoro-popliteal area is therefore important because relatively small pressure changes in the non-linear area will have considerable effect on $\mathrm{TT}$. It is therefore unlikely that normalization for blood pressure in the femoro-popliteal area is feasible, especialiy since the noninvasive measurement of mid-thigh or high-thigh pressures is still considered controversial $(3,31,35,40,60,85,148,150)$.

It lends great credit to the instrumentation and methods used by Gosling (69) (two transducers, spectral analysis, off-line foot-to-foot detection) that normalization of TT for blood pressure leads to improvement of his results. Humphries (77), on the other hand, reported no improvement of the separation between the arteriographic groups using an identical technique.

The observed low values of the correlation coefficients $(R=-0.17$ and -0.22$)$ express foremost the cumulative effect of measurement error or variation on

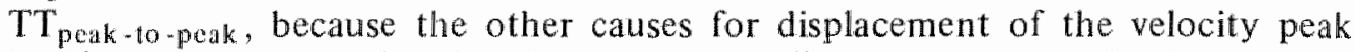
besides the vessel conduit length and vessel wall compliance can, be disregarded. The calculations were carried out on normal pulsatile velocity wave forms thus excluding damping as a possible cause for displacement of the velocity peak (fig. 3.6).

Gosling (68a) compared $\mathrm{TT}_{\text {foot-1o-foot }}$ with $\mathrm{TT}_{\text {peak-to-peak }}$ in the common femoral to dorsal pedal arterial pathway in normal supine subjects at different degrees of table tilting. $\mathrm{TT}_{\text {foot-to-foot }}$ decreased more or less linearly with increasing tilt, while $\mathrm{TT}_{\text {pcak-to-peak }}$ was unpredictable, which was explained by the velocity peak displacement following reflections at the peripheral end of the circulation $(68 \mathrm{a}, 116,117,159)$. The $\mathrm{TT}_{\text {peak-10-peak measurements in this study }}$ were far removed from the potential site of reflection in the normal individual, which make them an unlikely cause for the shown scatter around the regression line, since it is also known that maximum effect from reflections can be expected near the site of reflection $(102,111)$. To conclude, one may speculate that the theoretical improvement in the discriminative value of $\mathrm{TT}_{\text {peak-to-peak, }}$ resulting from normalization, will be obscured by relatively large patient-examiner or instrument-related causes of error and variation.

\subsection{The specificity of the hemodynamic parameters for femoro-popliteal lesions}

\subsubsection{Results}

The previous sections of this chapter established a statistically significant difference between the arteriographic groups (normal, stenosis, occlusion) for all hemodynamic parameters except Diam $\mathrm{SF}$ A -20 and Diampop (table II, appendix). None of the parameters, on the other hand, could individually classify a leg to a particular group as a result of the observed overlap (fig. 4.13 to fig. 4.19).

Discriminant analysis is especially useful in these situations because it makes optimal use of the variance as present over all parameters in order to obtain maximum discrimination. Several combinations of the parameters as described in 
section 4.7 were therefore subjected to discriminant analysis (section 3.8.1). The analysis was separately carried out with the parameters obtained with both instrumentations. The ankle systolic pressure indices before and after exercise (Index I and Index II) were also included.

- Pulsed Doppler instrumentation: damping between the common femoral and popliteal arteries $(\Delta c o m-p o p P D)$, damping between the deep femoral and popliteal arteries $(\Delta$ prof-poppd $)$, transit-time between the common femoral and popliteal arteries (TTPD) and the diameter estimations of the SFA at 20 $\mathrm{cm}$ 's below the inguinal ligament (Diam $\mathrm{SFA-20}_{20}$ ) and the popliteal artery (Diampop).

- CW instrumentation: damping and transit-time between the common femoral and popliteal arteries $\left(\Delta\right.$ com-pop $\mathrm{p}_{\mathrm{W}}$ and $\left.\mathrm{TT}_{\mathrm{CW}}\right)$.

The results of discriminant analysis with different combinations of these parameters are summarized in table 4.7 (for comment, see section 4.9.1.3).

\subsubsection{An example}

The results of discriminant analysis including $T_{P D}, \Delta c o m-p o p p D$ and $\Delta$ prof-poppD will be discussed in greater detail, serving as an example for the reader who is not familiar with this method.

The femoro-popliteal segment was à priori classified as normal $(0)$, stenotic (1) or occluded (2), according to the arteriographic classification as given in table 4.6. Classification with discriminant analysis determined the à posteriori allocation of each leg in the diagram (fig. 4.24A and B). Group 1 (stenosis) is overlapping with group 0 (normal) and group 2 (occlusion), while groups 0 and 2 seem to be almost completely separated in the diagram.

The observed overlap in the diagram can be interpreted as the remaining overlap after summary comparison and discriminant analysis of all parameters in each group. The almost complete disappearance of the overlap between groups 0 and 2, formerly shown in figures $4.14,4.16$ and 4.17 for each individual parameter, results from optimal use of variance by the calculated functions as supplied by discriminant analysis. Popliteal artery occlusions for example (c in fig. 4.14, $4.16,4.17)$ are usually associated with severe loss of pulsatility due to poorly developed collateral supply routes at this level of the arterial system. The TTPD of the velocity wave, on the other hand, is significantly less influenced, because the available collaterals are short. Discriminant analysis determined in this case that loss of pulsatility (damping) was far outweighing the apparently normal transittime.

Each leg is located in the diagram according to the 'à posteriori' calculation, and indicated with its 'a priori' classification. The legs in each group cluster around their centroid (mean) thereby demarcating their territory. Legs trespassing' their territory are incorrectly classified according to the a posteriori classification (fig. $4.24 \mathrm{~A}$ and B). All information regarcling the correctly or incorrectly classified legs is summarized in table 4.8 . The number of legs in each group actually used in the analysis, is at variance with the number given in table 4.6 following the occurrence of missing parameter values in some legs in all groups.

This table is of considerable interest because its way of presentation allows application of generally used analysis techniques. In this particular example $80.1 \%$ 


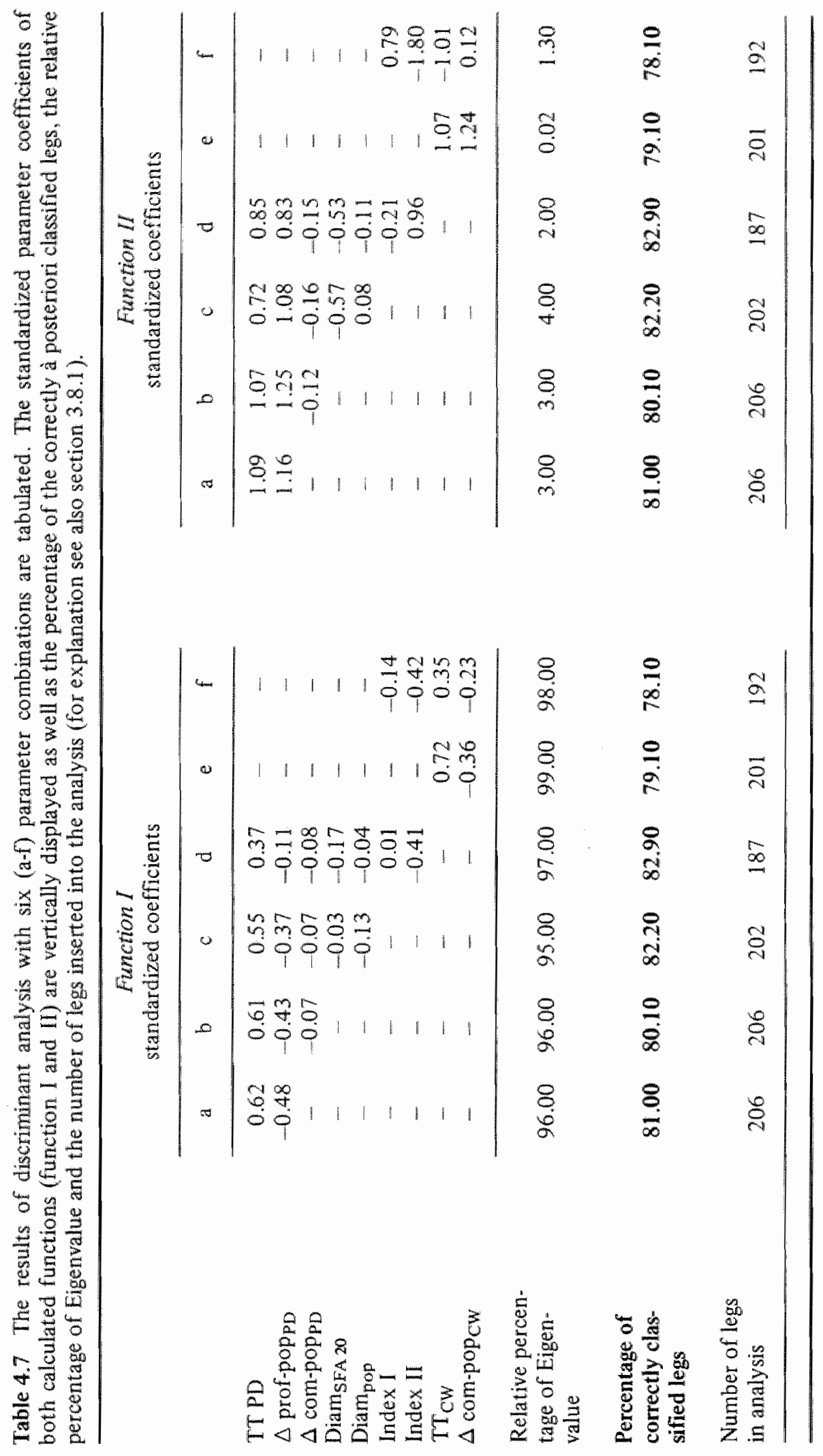



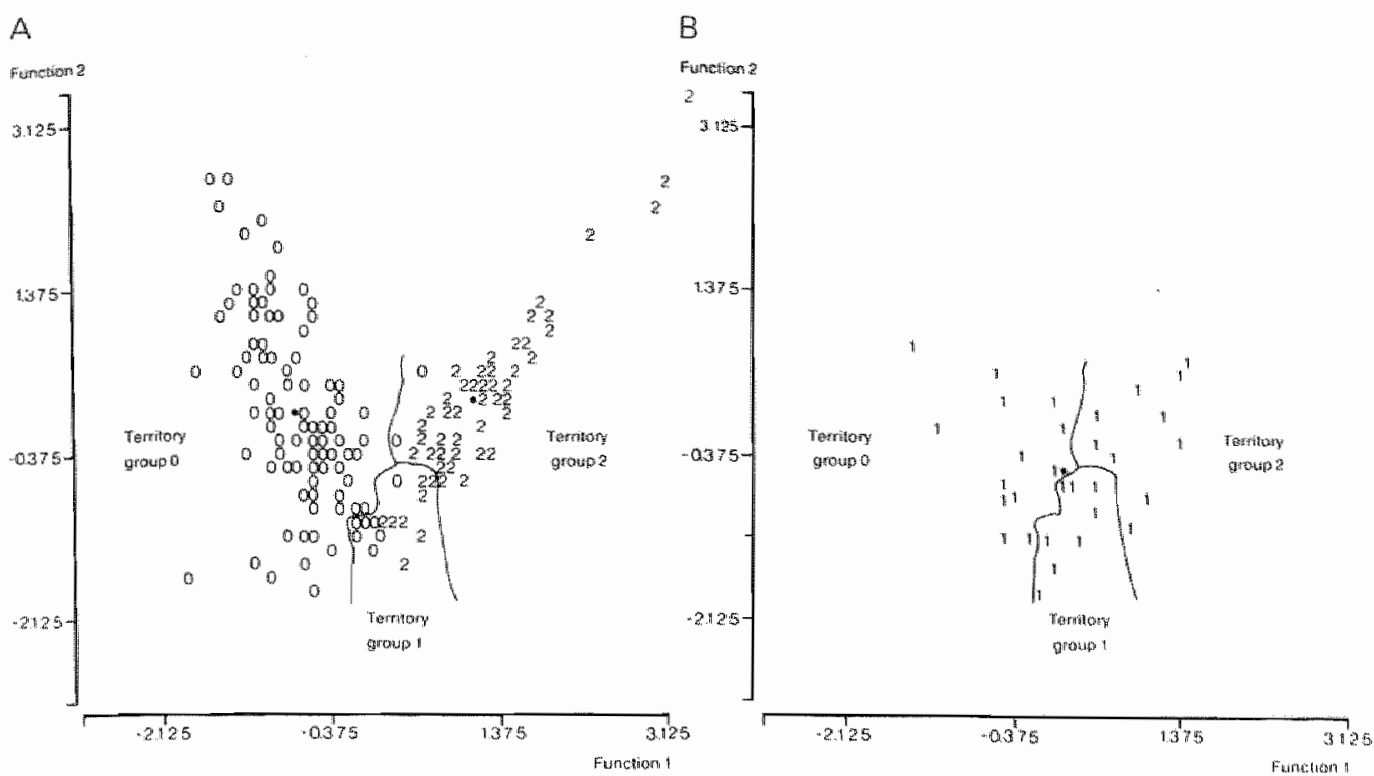

Fig. 4.24A and $B$ Two hundred and six legs $(0=$ normal, $1=$ stenosis, $2=$ occlusion $)$ are displayed, their à posteriori allocation in the diagram determined by the calculated value of function I (horizontal) and function 11 (vertical), both derived from discriminant analysis, using the coefficients (table 4.7 ) as weighting factors. Each leg is indicated with its a priori classification so that legs "trespassing' their territory can be easily identified. To prevent confusion two separate diagrams are made. The centroid (mean) of each group is indicated as *

of the legs were correctly classified (diagnostic accuracy), meaning that $19.9 \%$ were 'trespassing' their territories, most of them belonging to group 1 (stenosis). Specificity and sensitivity are statistical terms exclusively reserved for the dichotomous situation, so that the data in table 4.8 had to be re-arranged for their calculation.

The conversion of this three-way table (table 4.8 ) into 3 two-way tables (normal vs stenosis - occlusion; stenosis vs normal-occlusion; occlusion vs normalstenosis), needed for the calculation of specificity and sensitivity, is given in table III of the appendix. They range respectively from $32.4 \%$ to $91.8 \%$ and from $85.4 \%$ to $92.4 \%$.

A perhaps more adequate way to describe the agreement between two diagnostic methods, is to calculate the overall diagnostic accuracy (percentage of correctly classified legs) corrected for the marginals (M1-M6 in table 4.8). Kappa, a measure for pairwise agreement (137), is determined by the diagnostic accuracy (the diagonal in table 4.8) and the marginal distribution (M1-M6). Small changes in a three-way table e.g. following re-allocation of falsely classilied items (legs) may not influenced the diagnostic accuracy, but are always reflected by changes in the marginal distribution and thus expressed through Kappa. The Kappa-corrected diagnostic accuracy of table 4.8 is 0.66 , where 1.0 means complete agreement between two diagnostic methods and 0.0 absence of any agreement (for better appreciation see also table $\mathrm{V}$ in the appendix).

\subsubsection{The effect of changes in the à priori classification}

The criteria for à priori classification of the legs were strictly applied so that tight 
stenoses in the femoro-popliteal pathway, showing e.g. a razor-thin line of contrast, were allocated to group 1. Re-examination of the arteriograms with more realistic (clinical) interpretation of the visualized lesions, resulted in reclassification of 16 legs. Reasons for reclassification were, for example, generalized narrowing of the SFA or the post-operative ascertainment that a stenosis of the SFA, observed on a pre-operative arteriogram, was of no significance as deduced from complete normalization of the ankle pressures ariter aorto-iliac reconstructive surgery. Four legs, previously considered normal, were reclassified as stenosis and

Table 4.8 This three-way table displays the à priori classification (arteriography) vertically and the à posteriori classification (hemodynamic parameters) horizontally. Nine cells (A-I) are thus formed. The percentage of correctly classified legs (diagnostic accuracy) is calculated $\left(\mathrm{P}_{0}\right)$. The marginals $\left(\mathrm{M}_{1}-\mathrm{M}_{6}\right)$ are used for calculation of $\mathrm{P}_{\mathrm{c}}$ and subsequently for the calculation of the Kappa-corrected diagnostic accuracy.

\begin{tabular}{|c|c|c|c|c|}
\hline \multirow[b]{2}{*}{$\begin{array}{l}\text { a priori } \\
\text { (arteriography) }\end{array}$} & \multicolumn{3}{|c|}{$\begin{array}{c}\text { á posteriori } \\
\text { (hemodynamic parameters) }\end{array}$} & \multirow[b]{2}{*}{ Marginals } \\
\hline & $\begin{array}{c}0 \\
\text { (normal) }\end{array}$ & $\begin{array}{c}1 \\
\text { (stenosis) }\end{array}$ & $\begin{array}{c}2 \\
\text { (occlusion) }\end{array}$ & \\
\hline 0 (normal) & $\begin{array}{ll}\text { A } & \\
& 101\end{array}$ & B & C & $M_{1}$ \\
\hline 1 (stenosis) & 13 & 12 & $\mathbf{F}$ & $\mathrm{M}_{2}$ \\
\hline 2 (occlusion) & G & $\mathrm{H}$ & 52 & $M_{3}$ \\
\hline Marginals & $M_{4} \quad \begin{array}{l}115 \\
\end{array}$ & $\begin{array}{ll}\mathbf{M}_{5} & \\
& 28\end{array}$ & $M_{6}$ & ${ }^{N} 206$ \\
\hline
\end{tabular}

Percentage correctly classified legs : $\frac{A+E+1}{206}: 80.1 \%\left(P_{0}\right)$
(diagnostic accuracy)

Kappa-corrected diagnostic accuracy : $66.0 \%$ (see below)

$$
\begin{aligned}
& \text { Kappa }=\frac{P_{0}-P_{c}}{1-P_{c}} \quad P_{0}=\text { observed agreement }=\frac{A+E+1}{206}=0.8009 \\
& P_{c}=\left(\frac{M_{1}}{206} \times \frac{M_{4}}{206}\right)+\left(\frac{M_{2}}{206} \times \frac{M_{5}}{206}\right)+\left(\frac{M_{3}}{206} \times \frac{M_{6}}{206}\right)=0.4117 \\
& K=\frac{0.8009-0.4117}{1}=0.66
\end{aligned}
$$


12 legs, previously considered stenotic, were either reclassified as occlusion (8) or normal (4). The percentage of correctly classified legs rose from $80.1 \%$ to $87.7 \%$, when the analysis was repeated as described in the previous section.

\subsubsection{Discriminant analysis with different parameter combinations}

Discriminant analysis is a suitable tool to specify the value of the different hemodynamic parameters under investigation. It also provides the opportunity for numerical comparison of both Doppler instrumentations, with regard to their capacity to discriminate between lesions of increasing arteriographic severity.

By subjecting several parameter combinations to discriminant analysis, existing differences in variance between the parameters emerge through their standardized coefficients calculated for each function. These coefficients are of great analytical value because they control the actual contribution of each individual parameter to the final value of the respective functions (see formula in section 3.8.1). The value of a coefficient can be interpretated as being representative for the discriminative value of the corresponding parameter.

The results of discriminant analysis with several parameter combinations are summarized in table 4.7. Transit time ( $\mathrm{TT}_{\mathrm{PD}}$ as well as $\mathrm{TT}_{\mathrm{CW}}$ ) has greater discriminative value for femoro-popliteal disease than damping, irrespective of the Doppler instrumentation used. A similar systematic difference is also present between $\Delta$ prof-popPD and $\Delta$ com-pop $_{\mathrm{PD}}$, the latter consistently having a lower coefficient in each parameter combination. The parameters Diampop and Diam $_{S F A-20}$ are ranking third and their insertion into the analysis increased the percentage of correctly classified legs only from $80.1 \%$ to $82.2 \%$. No significant contribution towards better discrimination between the arteriographic groups is given by these parameters. Diam SFA -20 reached a relatively high value only in the second function $(0.57)$.

The high values of the coefficients belonging to the pressure indices need some clarification. It has been shown (section 4.4) that pressure indices at the level of the ankle detect the presence of arterial disease with a high degree of accuracy, but also that their ability to differentiate between different localizations of disease is poor. The pressure indices (especially Index II in parameter combination d) are thus powerful discriminating parameters for normal (group 0 ) and diseased legs (groups 1,2), apparently at least as good as $\mathrm{TT}_{\mathrm{PD}}$ and $\mathrm{TT}_{\mathrm{CW}}$. The large value of their coefficients is to some extent explained by the relatively large number of normal legs included in the analysis.

Important is the observation that corresponding parameters obtained with both Doppler instrumentations discriminate equally well between the three arteriographic groups. The difference is slight and in the order of $1-2 \%$ (table 4.7 ).

\subsubsection{Full-length occlusion of the SFA}

Considering the hemodynamic consequences of long and short occlusions of the SFA one expects in general a greater loss of function with full-length occlusions of the SFA (162).

Legs with full-length occlusion of the SFA were therefore distinguished from those with short SFA occlusions and the resulting four groups (normal, stenosis, short occlusions, full-length occlusions) were subjected to discriminant analysis with four parameter combinations. Table 4.9 lists the percentages of correctly and incorrectly classified legs for each arteriographic group (0-3) over four parameter 
combinations (be) used in the previous section. The coefficient of each parameter in all parameter combinations is given in table IV (appendix).

In spite of substantial overlap between both occlusion groups $(2,3)$, legs with full-length occlusions are separated better from normal and stenotic legs than legs with short occlusions, which is most marked when all parameters are included in the analysis (table 4.9 , combination d). This interpretation is more conveniently visualized in fig. 4.25 depicting a cluster of legs from group 3 in the lower right corner of the diagram. TTPD, Diam $\mathrm{TFA}_{20}$ and Index II attained the highest discriminant coefficients in combination d (table IV, appendix). Comparison with table 4.7 permits the tentative conclusion that the diameter measurement of the

Table 4.9 The information from four 4-way tables (b-e) is summarized. Each 4-way table depicts the percentage of incorrectly and correctly à posteriori classified legs for one particular parameter combination over four arteriographic groups (0-3). The four parameter combinations $b, c, d$ and $e$ are described in detail in table 4.7. The arteriographic groups are $0=$ normal, $1=$ stenosis, 2 =occlusions in the fernoro-popliteal segment with exclusion of full-iength occlusions of the SFA, 3 = full-length occlusions of the SFA. The percentage of correctly classified legs (diagnostic accuracy rate) is given in the lower right corner. Between ( ) Kappa.

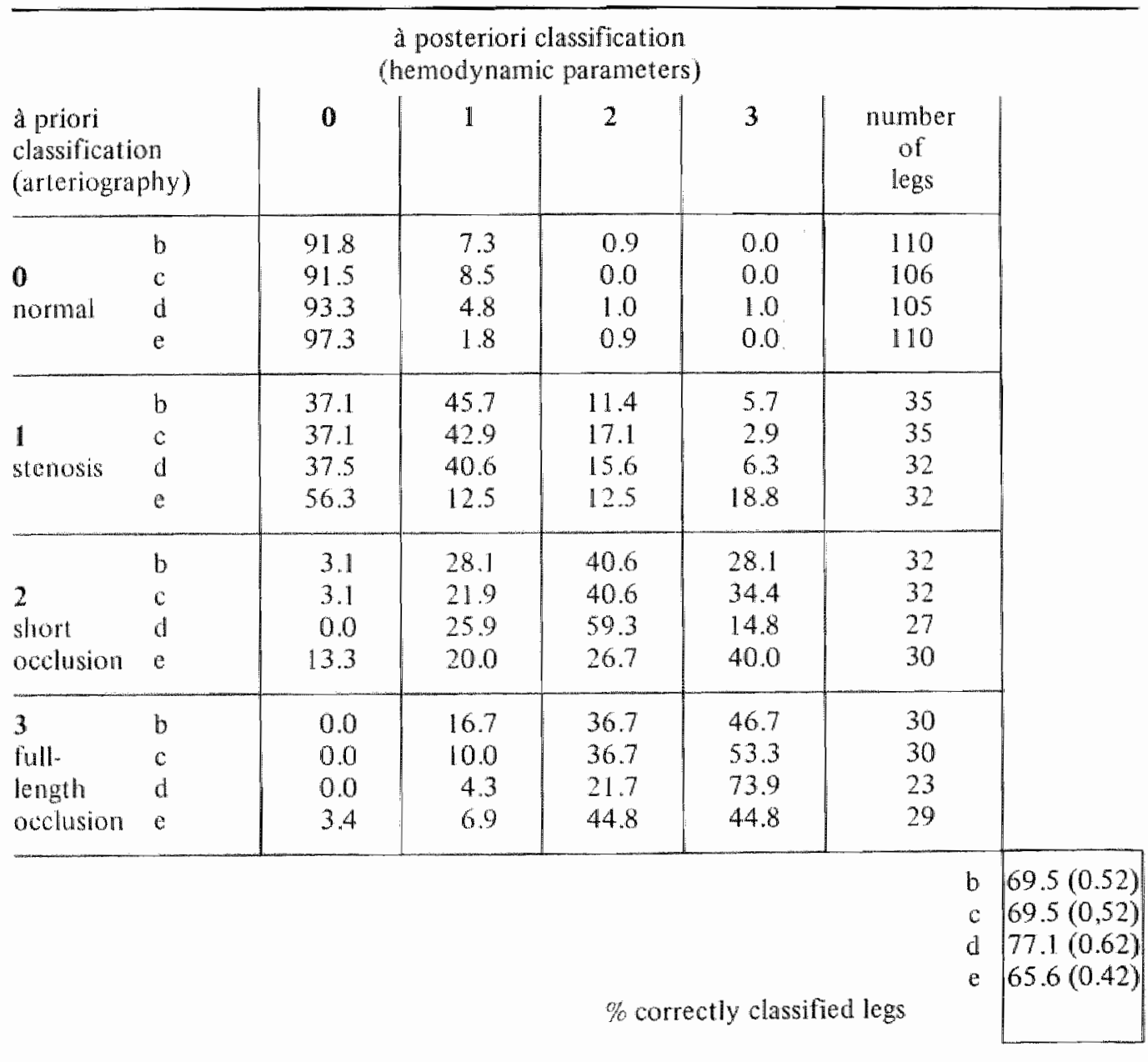


SFA at $20 \mathrm{~cm}$ 's below the inguinal ligament contributes significantly to the observed discrimination between full-length and short SFA occlusions. This is is not surprising because full-length occlusions of the SFA are reliably picked up with the multi-gate pulsed Doppler system (section 4.3).

\subsubsection{Validation of discriminant analysis}

Good practice in statistical analysis demands that definition and subsequent testing of criteria is done independently. Although à posteriori classification in discriminant analysis is performed after calculation of the standardized discriminant function coefficients, analysis and classification, comparable to definition and testing, are clearly not truly independent.

A random sample containing $50 \%$ of the available legs $\mathrm{B}_{\mathrm{F}}$, was therefore subjected to discriminant analysis, and the calculated functions were used to classify the legs in the remaining other half (A). In this way, comparison was

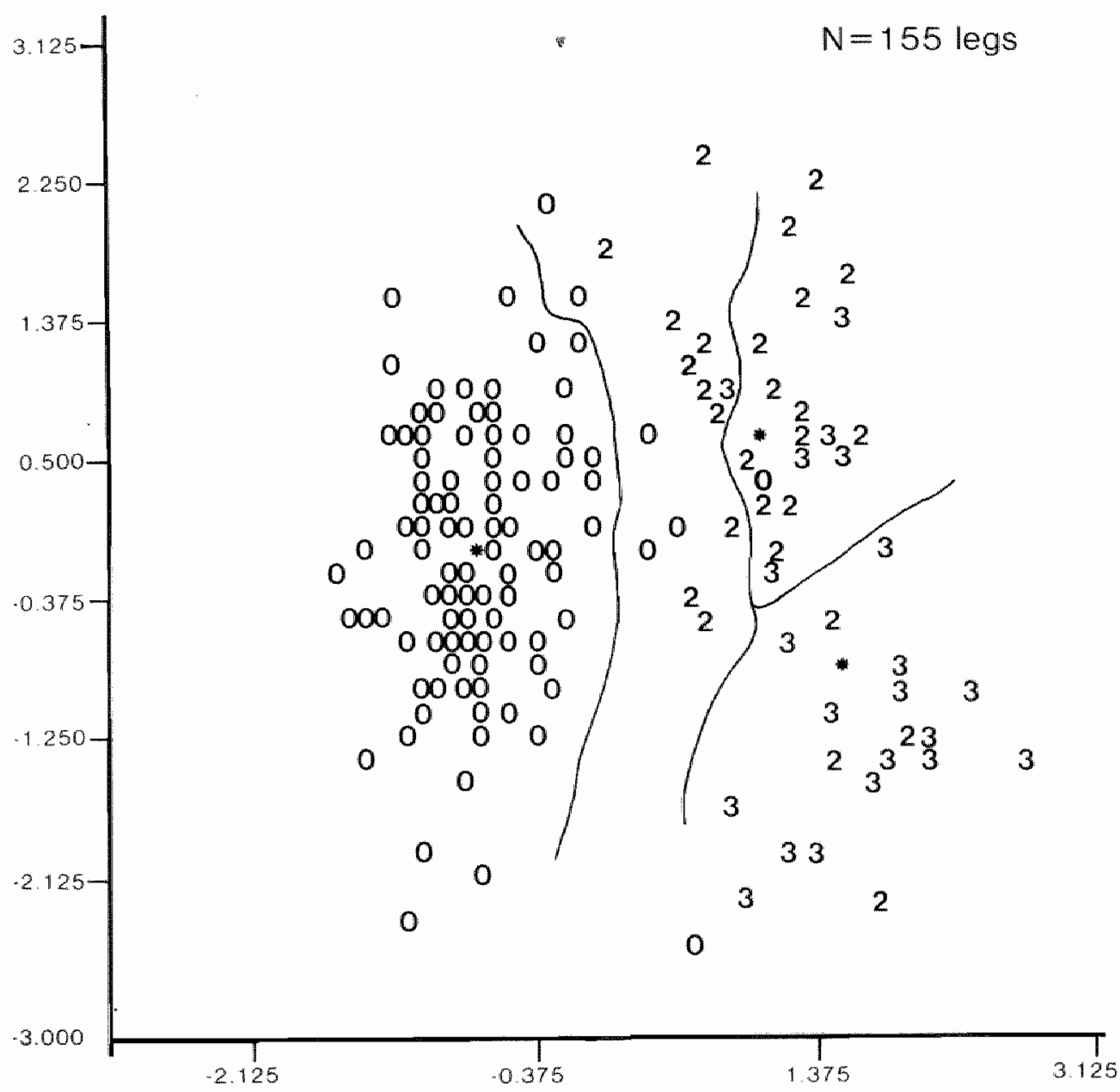

Fig. 4.25 Hundred and fifty-five legs $(0=$ normal, $2=$ short occlusion SFA, $3=$ full-length occlusion SFA) are displayed in a diagram according to the calculated value of functions $\rrbracket$ and II of combination d (see also fig. 4.24A and B). Legs with full-llength occlusion (3) cluster in the right lower comer, their centroid $\left({ }^{*}\right)$ further removed from the normal legs. 
Table 4.10 Each column denotes the percentage of correctly classified legs in samples $A, B$, $A_{\mathrm{F}}$ and $\mathrm{B}_{\mathrm{F}}$ calculated for six parameter combinations (a-f) as previously defined in table 4.7 . Discriminant functions are calculated in one randomized sample $\left(B_{F}\right.$ or $A_{F}$ ). The remaining legs (samples $A$ or $B$ ) are classified with these computed functions $\left(A-B_{F}\right.$ or $\left.B \leftarrow A_{F}\right)$. The percentage of the correctly à posteriori classified legs (in samples $B_{F}$ or $A_{F}$ ) are also given vertically in line of the headings $\mathbb{B}_{\mathrm{F}}$ and $\mathrm{A}_{\mathrm{F}}$.

\begin{tabular}{lllll}
\hline $\begin{array}{l}\text { Parameter } \\
\text { combinations }\end{array}$ & \multicolumn{2}{l}{ samples } & \multicolumn{2}{l}{ samples } \\
& $\mathrm{A} \leftarrow \mathrm{B}$ & $\mathrm{B} \leftarrow \mathrm{A}$ \\
\cline { 2 - 4 } $\mathrm{a}$ & 78 & 82 & 86 & 79 \\
$\mathrm{~b}$ & 78 & 82 & 84 & 79 \\
$\mathrm{c}$ & 72 & 83 & 82 & 80 \\
$\mathrm{~d}$ & 78 & 86 & 81 & 86 \\
$\mathrm{e}$ & 74 & 83 & 83 & 77 \\
$\mathrm{f}$ & 74 & 85 & 76 & 81 \\
\hline
\end{tabular}

possible between the percentage of correctly classified legs from sample $A$, assessed with discriminant functions derived from sample $\mathrm{B}_{\mathrm{F}}$ with the percentage of correctly à posteriori classified legs in sample $B_{\mathbb{T}}$. This was done for all combinations of the parameters already described in section 4.9.1.3. The same procedure was carried out in reverse order and the results are summarized in table 4. 10 .

Small differences are observed between the percentage of correctly classified legs as determined in samples $A$ and $B_{F}$ (à posteriori) as well as between $B$ and $A_{F}$ (à posteriori). The differences were generally similar in magnitude and sign following both procedures $\left(A \leftarrow B_{F}\right.$ and $B \leftarrow A_{F}$ ). Random samples taken from a limited population (approximately $200 \mathrm{legs}$ ) are not likely to have an identical composition, which may explain the small differences as observed in table 4.10. This verification technique firmly establishes discriminant analysis as a reliable method for the discrimination of atherosclerotic lesions in the femoro-popliteal pathway.

\subsubsection{Discussion}

Discriminant analysis is a popular statistical tool in behavioural sciences. Interest in this technique by members of the medical profession $(31,112)$ who are faced with vast quantities of data has been noted to increase. Discriminant analysis is particularly useful in the present study because the value of each hemodynamic parameter can be individually assessed. Parameters with high discriminative value can be identified so that less valuable parameters can be eliminated. This leads to a reduction of data but also towards a better insight into the problem.

Analysis of the standardized discriminant function coefficients corroborated, to a certain extent, the earlier findings in the previous sections. The TT peak-to-peak of the velocity wave is the best parameter for discrimination between arteriographically defined groups of disease in the femoro-popliteal pathway irrespective of the used instrumentation. It indicates that the on-line detection of the peak of the analog velocity wave form, using the $\mathrm{R}$-wave of the ECG as a reference, is 
clinically relevant and of sufficient accuracy to establish $\mathrm{TT}_{\text {peak-to-peak mea- }}$ surements as a hemodynamic parameter of practical significance. All damping factors have consistently lower coefficients than those of the transit-time. TT and damping together embrace nearly all the available variance in each arteriographic group for all hemodynamic parameters so that additional parameters (e.g.diameter), can hardly contribute to better separation. The coefficient value of Index II and $\mathrm{TT}_{\mathrm{PD}}$ (combination $\mathrm{d}$, table 4.7 ) are almost equal, which might be explained by the high discriminative value of pressure measurements at the level of the ankle for normal legs or legs with arterial disease (section 4.4.). The total number of correctly classified legs hardly increases, so that the conclusion is justified that TT and damping of either instrumentation does this equally well.

Physiological reasoning may explain the high discriminative value of TT peak to-peak. The maximum velocity peak of the analog velocity wave form may be subjected to temporal displacement either away from, or towards the referent R-wave of the ECG, respectively caused by longer conduit vessels $(69,70)$ and damping or by reflected pressure waves $(68 \mathrm{a}, 102)$. A longer vascular pathway and damping effectuate displacement of the systolic peak at the distal site of mea-

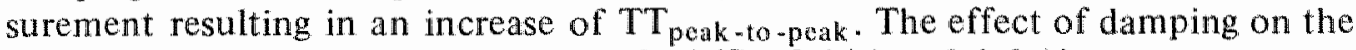
peak displacement is usually quite marked (fig. 3.11 A and 4.6.A).

The direction of the systolic peak displacement caused by reflected velocity waves, on the other hand, is towards the reference. Reflections in the femoral area are strong in the presence of occlusive disease in the SFA, while the peripheral impedance distal to significant disease is usually lowered causing few reflections $(53,102)$ to occur in this area. It is therefore argued that $\mathrm{TT}_{\text {peak-to-peak will }}$ increase following the occurrence of reflected waves in the femoral artery. Reflections enhance the discriminative value of $\mathrm{TT}_{\text {peak-to-pcak }}$ when the SFA is occluded.

The great merit of discriminant analysis concerns its ability to provide a formula aimed at maximal separation of previously (à priori) defined groups. The verification procedure (section 4.9.1.5) effectively eliminates hindsight from contributing to the observed percentage of correctly classified legs. The statistical validity of discriminant analysis is thus affirmed, which will permit practical application of such methods. Discriminant scores calculated from a representative population of patients with intermittent claudication would be able to classify any newly presented leg into one of the defined groups with a 'reasonable' degree of accuracy.

How reasonable is 'reasonable'? TT and damping together were able to distinguish successfully between the arteriographic groups in approximately $80 \%$ of the legs included in the analysis, regardless of the instrumentation used. This degree of accuracy seems low if compared to the high levels of specificity and sensitivity noted with other noninvasive methods (114).

Specificity (the percentage of true negatives recognized by a test) and sensitivity (the percentage of true positives that are recognized by a test) are valid statistical terms when applied to a dichotomous situation. It explains the popularity of these terms in relation to the many noninvasive tests currently used for the detection of carotid disease. Clinicians are mainly interested in identifying the degree of stenosis at which further diagnostic investigations or even surgery must be contemplated, so that a single value of a test is selected (threshold level) above which the test is considered positive. 
This is obviously not possible in the present study because three categories of disease are recognized (normal, stenosis and occlusion). It can easily be seen that none of the current statistical terms (specificity, sensitivity, false negative, false positive) have meaning for the three-way table, as presented in table 4.8 . Its modification (table III, appendix), necessary in order to calculate these statistical terms, pictures their metaphorical use and does not compare to the more refined 'à priori" and 'à posteriori' classification offered by discriminant analysis.

It was stated by $O^{\prime}$ Donnell (114) 'that the overall diagnostic accuracy (all correct results/all tests performed) depends on the prevalence of disease in the study population and is a poor measure of a noninvasive test." This statement is of value* but when noninvasive tests are discussed with diagnostic capabilities going beyond dichotomous discrimination, diagnostic accuracy (or the percentage of correctly classified legs) remains the only meaningful term left for its statistical evaluation. It is therefore proposed to apply Kappa (137) because it corrects for the marginal distribution and 'normalizes' the found overall diagnostic accuracy for differences in composition of the patient group under investigation. The Kappa-corrected diagnostic accuracy allows better comparison of the results from different noninvasive studies. Of course, data need to be complete in order to do so.

This study identified TT and damping as the best discriminating parameters, so that comparison with Gosling's work $(67,69)$ would be natural and justified. Apart from noting a firm degree of similarity between his linear representations, using $\mathrm{TT}_{\text {foot }-\mathrm{to} \text {-foot }}$ and damping for the differentation of four arterial conditions (normal, mismatch, stenosis, occlusion), and the graphical display, resulting from discriminant analysis as given in figure 4.24 , nothing more can be said as a result of differences in the analysis technique. The Kappa-corrected overall diagnostic accuracy would have been an ideal statistical measure for comparison in this situation. Its calculation from the findings as presented in the literature is unfortunately not possible because complete data are lacking.

The functional deficit resulting from an atherosclerotic lesion in the SFA is related to the extent and severity of the lesion but also to the quality of the collateral response. The leg with a full-length occlusion of the SFA will depend completely on collateral supply routes fed by the deep femoral artery. Short occlusions of the SFA allow shorter, additional collaterals, originating from the SFA itself, to compensate for the loss of function. It is thus to be expected that full-length SFA occlusions will cause greater functional deficit than short occlisions (162). The hemodynamic parameters as well as the applied analysis technique seem sensitive enough to visualize this expected difference in a scattergram. Legs with full-length occlusions of the SFA are further away from normal legs on the scattergram than legs with short occlusions. These results appear to be in agreement with the findings of Gosling $(69)$ and Fitzgerald $(56,57)$ in so far as differences in the quality and length of collaterals can be expressed with the hemodynamic parameters at hand. The 'clustering' effect of legs with full-length occlusions of the SFA is most pronounced (fig. 4.25) when all available parameters are inserted into the analysis (combination d). That the diameter estima-

* It can easily be proven that the overall diagnostic accuracy is a function of specificity, sensitivity and the number of discased legs. 
tion of the SFA at $20 \mathrm{~cm}$ 's below the inguinal ligament is a relatively strong discriminator refers to its accuracy to diagnose full-tength occlusions. Although one would be tempted to analyse legs with combined disease of the SFA and the deep femoral artery, in relation to the remaining legs, the temptation was resisted in view of the unreliability of arteriographic reading in this area $(14,29,95)$ as discussed in section 2.2 .

The summary results of the present study have been discussed in this section. They showed that $\mathrm{TT}_{\text {peak }}$ to -peak and damping, whether obtained with the $\mathrm{CW}$ or pulsed Doppler system, are capable to describe function and loss of function in the femoro-popliteal pathway with sufficient accuracy, allowing the correct identification of three different arteriographic groups in $80 \%$ of the cases. These results are in general agreement with the findings of Gosling (69) and Humphries (77), although meticulous comparison is impossible owing to differences in the analysis technique. The calculation of the Kappa-corrected diagnostic accuracy is proposed to facilitate future comparison with other studies.

The ability of discriminant analysis to respond to small changes in the "à priori' classification (section 4.9.1.2.), added to the results of the statisticall validation (section 4.9.1.5), established the usefulness of this statistical technique.

That the localization of an individual leg in the 'butterfly' shaped figure, presented with discriminant analysis, corresponds to the functional behaviour of its entire femoro-popliteal system including the collateral bed, is a tentative conclusion given by the comparison of full-length and short occlusions of the SFA. 


\subsection{General discussion and conclusions}

The main purpose of this study has been to evaluate and compare the efficacy of a set of hemodynamic parameters obtained in the femoro-popliteal vascular segment for the functional description of atherosclerotic lesions in this area. The femoro-popliteal pathway was selected as the target area for investigation because good ultrasonic access is offered by arteries in the high femoral and popliteal area's.

The conceptual simplicity of pulsatility index and transit-time as noninvasive parameters of disease, introduced by Gosling et al in 1969 (70), has been of considerable appeal $(56,57,73,77,80,81)$. However, the costly and rather complex implementation of the originally proposed method may have limited its widespread use. A system for on-line computation of PI and TT was therefore developed (138) and tested in clinical surroundings, aiming at low costs, reliability and a practical way of operation. An on-line method can be of relatively simple design if an analog voltage signal is employed, so that some of its objectives, llow cost and practicability, are fulfilled with the use of a commercially available $\mathrm{CW}$ Doppler instrument.

Sharp and clearly defined voltage changes are required for the on-line measurement of TT, so that it is difficult to calculate TT from the slow rising foot of the wave form. The delay-time between the R-wave of the ECG and the arrival of the systolic velocity peak at a proximal and distal site of the femoro-popliteal pathway was therefore determined, so that $\mathrm{TT}_{\text {peak-to-peak }}$ could be calculated by subtraction of both delay-times (sections 3.4 and 3.6). The R-wave of the ECG wals also used to trigger beat-to-beat computation of PI from the velocity-time wave form.

Gosling et al $(67,69,70)$ proposed the simultaneous measurement of P1 and $\mathrm{TT}_{\text {foot-to-foot }}$ at two arterial sites in order to reduce the influence of time-related phenomena in the arterial system (e.g. cardiac arrhythmia, respiration). Two operators are obviously needed for these simultaneous measurements, which is neither practical nor cheap. A single transducer was therefore employed in this study, so that proximal and distal measurements in the leg were separated in time. To correct for the instrumental error introduced by the wave form analyser, but also to reduce the influence of the time-related phenomena, averaging of PI and DT peak - to -peak was performed over 20-30 consecutive heartbeats (sections 3.6 and 3.7). This brief summary of the followed methods, different in almost all aspects from the methods formerly introduced by Gosling $(64,66,67,68,68 \mathrm{a}, 69,70)$, is necessary to fully appreciate the significance of the findings in chapter four (table 5.1).

Spectral analysis is generally regarded $(56,67,73,77,80,170)$ as the superior method for processing of the audio signal because it permits reliable interpreta- 
Table 5.1 List of most important differences between materials and methods of the present study (left) and such as used by the Guy's group (right) $(64,66,67,68,68 \mathrm{a}, 69,70$ ).

zero-crossing meter

one transducer

on-line parameter calculation

reciprocal value of damping $(20-30$ heartbeats $)$

TT peak-to-peak $(20-30$ heartbeats $)$

no "normalization" for blood pressure

three arteriographic groups

discriminant analysis

Kappa-corrected diagnostic accuracy

femoro-popliteal pathway spectral analysis

two transducers

off-line parameter calculation

damping ( 3 heartbeats)

$\mathrm{TT}_{\text {foot -to-foot ( } 10 \text { heartbeats) }}$

"nomalization" for blood pressure

four arteriographic groups

linear representation

sensitivity, specificity

3 arterial segments ( $\mathrm{Al}$; fem-pop; pop-tib.)

tion of the Doppler signal in spite of poor signal-to-noise ratio's, venous velocity contamination, disturbed flow patterns and artefacts of various origin. A frequency-to-voltage converter reduces a spectrum of Doppler frequencies to a single voltage which is proportional to the true instantaneous mean frequency in the Doppler signal under ideal signal-to-noise conditions if the frequency spectrum is narrow $(6,69,80,94,123,129)$. Proponents of spectral analysis use the wellfounded theoretical argument that these optimal measuring conditions are seldom accomplished and that the output of a zero-crossing velocity meter is therefore likely to be at variance with the true instantaneous mean Doppler frequency. On the other hand, favourable reports regarding the reliability of these simple devices under clinical conditions, have recently been published $(60,139)$.

Most authors, favouring spectral analysis, use the maximum frequency outline of the sonagram to calculate PI under the assumption that the pulsatile behaviour of the centerstream high velocity laminae is proportional to the 'mean' pulsatility of the entire velocity profile (170). This assumption is permitted if the profile is either parabolic or flat, which is probably not the case during long periods of the cardiac cycle, as may be concluded from observations of the constantly changing profile. There is also increasing evidence that parabolic or flat profiles are rare in patients with atherosclerotic disease because spectral broadening occurs at relatively mild degrees of narrowing $(19,20,21,124)$, especially in the vicinity of atherosclerotic plaques of the vessel wall. It is thus not clear if PI of the maximum frequency outline of the sonagram is proportional to PI of the true instantaneous mean Doppler frequency.

No noninvasive method at present is able to measure accurately the true instantaneous mean velocity across the artery diameter, so that the inferred deficiency to calculate the 'mean' pulsatility index for pulsatile velocity flow across the entire artery, with either method of signal processing (O-crosser or spectral analysis), must be investigated in a manner agreeing with the present technical possibilities. The performance of a CW and a pulsed Doppler instrument were therefore compared, because the small frequency band of the centerstream pulsed Doppler signal would be theoretically not unsimilar, after zero-crossing, to the maximum frequency outline of the sonagram. The output of its zero-crossing meter should be less vulnerable to the influence of vessel wall movements or venous velocity contamination and the narrow frequency band would also assure 
proportionality of the output-voltage with the instantaneous mean Doppler frequency in the sample volume.

The comparison of both instrumentations by means of correlation studies of corresponding PI and DT values did not provide a conclusive answer as to which was the better functioning instrument (section 4.1). No systematic differences were observed between PI and DT measured with both systems, justifying the conclusion that, under clinical conditions of measuring, a zero-crosser is less sensitive to the effect of spectral broadening, venous contamination and vessel wall movements, than previously assumed. If one accepts the statement that the PI determination on centerstream pulsed Doppler measurements is comparable to the PI calculation on the maximum frequency outline of a sonagram, these results do suggest indeed that the theoretical limitations of the zero-crossing meter are of less importance under clinical conditions.

The substantial error or variation, related to the patient or to the examiner (section 3.7) is thought to be mainly responsible for the low degree of correlation between the on-line Pl measurements with both Doppler systems (section 4.2.). Sudden step-wise changes in this group of patient-or examiner-related variations, caused by movements, are assumed to be of greater importance than the timerelated factors, such as respiration or cardiac arrhythmia. These latter factors are responsible for undulating changes in PI and DT with the patient at rest, so that averaging over 20-30 heart cycles effectively reduces their disturbing influence and thus the variation. The effect of sudden step-wise changes in PI and DT can be eliminated to some extent by continuous monitoring of their displayed (wave form analyser) beat-to-beat value, which is impossible if only three PI and ten TT values are computed off-line (69). It is postulated that the on-line measurement of PI and TT, employing one transducer and averaging over 20-30 consecutive heartbeats, is as effective for the elimination of time-related causes of variation as the off-line computation of these parameters on selected wave forms, obtained with two transducers.

This postulate is supported by the efficacy of the hemodynamic parameters, thus obtained, to discriminate between three arteriographic groups in the femoropopliteal segment. The found diagnostic accuracy appears to be grossly similar to the findings as presented by Gosling (69) (section 4.9) although minute comparison was impossible owing to differences in the employed methods and lack of complete data. The accuracy rate of $80 \%$ (percentage of legs correctly classified), reached with both instrumentations, was satisfying.

The similar performance of both instrumentations does not imply that the use of the CW instrument is preferred, because the anatomic information obtainable with the multi-gate pulsed Doppler instrument can be of great value e.g. to identify a full-length occlusion of the SFA (sections 4.9.1.4 and 4.3). The diameter estimation of the arteries in the upper leg and knee is surprisingly accurate when correlated with the measured arteriographic diameter (section 4.3). Some examples (fig. 3.7A and $\mathrm{B}, 4.3,4.4,4.6 \mathrm{~A}$ and $\mathrm{B}$ ) illustrate that the detection of a stenosis is easy without B-mode or velocity imaging because good spatial orientation is provided by the system with the simultaneous display of 32 channels.

Eighty percent of the legs were correctly classified as normal, stenosis or occlusion. Comparison with the efficacy of other noninvasive techniques through 
specificity and sensitivity calculations is not possible because these terms are exclusively reserved for the dichotomous situation (section 4.9.2). It is therefore proposed to use the Kappa-corrected diagnostic accuracy (table $V$, appendix) because it corrects for the composition of a particular group of patients. More important even is its ability to express different degrees of false classifications. It is to be expected that the current popularity of specificity and sensitivity will diminish as the noninvasive methods become more discriminative and subtle.

Overlap ( $20 \%$ of the legs were incorrectly classified) is found in every study in which noninwasive parameters are compared to arteriographic findings.

PI measured at a given arterial site is dependent on the condition of the proximal arterial system (stenosis or occlusion) but also on the terminal impedance of the peripheral outflow system $(53,140,141)$. The results from experimental work on dogs (53) seem to be in close agreement with clinical findings $(73,80,81)$ that tight proximal stenoses always give rise to a low PI, whereas a wide range of PI values is found distal to mild stenoses, owing to the variability of the terminal impedance in these case.

In an unimpeded arterial segment, damping (defined as the difference in PI between two arterial sites) neutralizes the effect of lesions proximal to the proximal measurement as well as distal to the distal measurement. Pl, determined in the common femoral and the popliteal arteries, will be equally aflected by aorto-iliac lesions as by the terminal impedance. The results of this study confirm that aorto-iliac lesions do not influence damping determined in the femoropopliteal area (section 4.5), whether disease in the femoro-popliteal pathway is present or not. However, less clear is the influence of the terminal impedance on damping in the case of a significant atherosclerotic lesion in this vascular segment, because the input impedance at the proximal site of measurement is predominantly determined by the femoro-popliteal lesion, whereas the distally determined PI will be affected by a variable terminal impedance.

Reflections of pressure waves have been shown to influence the shape of the velocity wave form, and thus $\mathrm{PI}$, in the vicinity of significant obstruction in the arterial pathway $(13,111,112,117,159)$. The higher discriminative value of $\Delta$ prof-poppD as compared to $\Delta$ com-poppo (section 4.9.1.3) is tentatively explained by the fact that the reflected pressure waves hardly reach the deep femoral artery (fig.3.11A). In 30\% of the cases with an occluded SFA, wave form distortions of the common femoral artery were seen as a result of reflected pressure waves (section 4.6).

The variable terminal impedance as well as reflections may be considered as causes for overlap of damping between the arteriographic groups in the femoropopliteal area. It remains to be seen whether this overlap will disappear following future improvement of methods and instruments and it is thought that their refinement will only result in more reliable and accurate measurement of PI without affecting the intrinsically present overlap, determined by the variable terminal impedance and by the wave form distortion caused by reflections.

Gosling et al (70) introduced TT foot-to-foot as a second parameter, more or less independent of damping, to improve separation between arterial segments with increasing severity of atherosclerotic disease. Very little is known about the relation of $\mathrm{TT}_{\text {foot-to-foot }}$ and $\mathrm{TT}_{\text {peak-to-peak }}$ in health and in disease, although it 
seems certain that TT peak-to-peak is influenced by damping. No systematic differences were found between the delay-time as determined with the $\mathrm{CW}$ and pulsed Doppler instrumentations (section 4.2). This good correlation was interpreted as a sign that patient- and examiner-related sources of variation are of less significance on the measurement of DT peak-to-peak than on P.I. Discriminant analysis ident:fied TT as the best discriminating parameter for disease in the femoro-popliteal pathway (section 4.9.1.3) for which several physiological explanations were given (section 4.9.2), which comes in addition to the measurement accuracy to detect the systolic peak. As with damping, considerable overlap of TT was found between the three arteriographic groups (section 4.7 ).

Gosling et al $(67,69)$ described a beneficial effect on the discriminative value

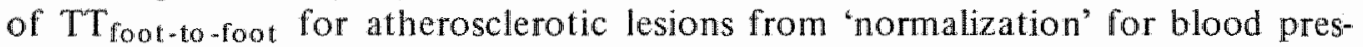
sure (mean). The results in section 4.8 suggest some kind of relation between

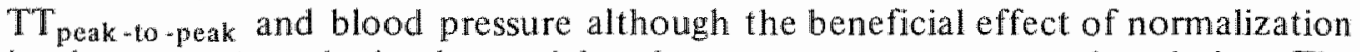
in the present study is obscured by the measurement error and variation. The maximum influence of normalization is to be expected in the lower pressure range (figure 4.22), e.g. distal to aorto-iliac disease, reducing its practical value, because of experienced difficulties with the accurate noninvasive measurement of pressure in the thigh region $(60,85,148)$. Humphries (77), employing the same methods as Gosling, was not able to show that normalization resulted in improved noninvasive separation of arteriographically dlefined groups in the femoro-popliteal area.

Two further subjects remain to be discussed in relation to the shown overlap between the arteriographic groups. Section 4.9.1.2 emphasizes the importance of correct arteriographic classification, which was illustrated by the effect of arteriographic reclassification of $16 \mathrm{legs}$ on the accuracy rate of discriminant analysis. The perilous use of arteriography as the 'Gold-standard' for comparison and validation of noninvasive parameters is increasingly recognized. Recently, a number of studies has pointed at the limitations of arteriography through interobserver variability studies $(29,46,176)$, as well as through comparison with intra-arterial pressure (107). Arteriography is undoubtedly useful for recognition of gross vascular abnormalities in patients with symptomatic atherosclerotic disease, but it is unable to match the subtlety of the hemodynamic parameters with values distributed over a wide, but continuous, scale. Grading of arteriographic lesions into three groups implies the separation of atherosclerotic lesions into three clear-cut, well-defined groups of disease, which is obviously not the case. The inherent simplification introduced by stratification of atherosclerotic lesions leads to the unavoidable loss of subtlety. The overlap between a set of continuous variables (e.g. hemodynamic parameters in the present study) is equally unavoidable because it is compared to a discontinuous, rigid Gold standard". The "butterfly" produced by discriminant analysis may be interpreted as a good representation of the spectral nature of the functional impairment of femoro-popliteal disease. Further investigations are necessary to confirm its practical usefulness for the recognition of improvement or deterioration of function in this wascular segment. 
In conclusion,

- the alleged inferiority of the zero-crossing meter, used as an instrument for quantification of the Doppler signal preparatory to calculation of damping and TT, is not sustained by the results of this study.

- the on-line measurement of PI and DT peak-to-peak from the analog velocity signal, employing one transducer and averaging the parameter values over 20-30 consecutive heartbeats, is probably as effective for the elimination of time-related causes of variation as the off-line parameter extraction from a limited number of sonagrams. This establishes the practicability of the method.

- the anatomic information supplied by the multi-channel pulsed Doppler system, as developed in Maastricht, is of great value owing to its ability to measure the arterial diameter, and to sample with certainty in pre-selected arterial sites (e.g. the deep femoral artery). The ultrasonic diameter estimation when combined with display of the velocity profile, is highly effective for the recognition of localized atherosclerotic disease in the femoro-popliteal pathway. Full-length occlusions of the superficial femoral artery are accurately diagnosed in $72-84 \%$.

- no improvement of the discriminative value of $\mathrm{TT}_{\text {peak -to }}$ peak is to be expected from 'normalization' for systolic pressure in the femoro-popliteal pathway. reflections of the velocity wave from significant atherosclerotic lesions in the SFA have a harmful effect on the discriminative capacity of damping for lesions in the femoro-popliteal segment. The damping factor, calculated between the deep femoral and popliteal arteries, is less influenced, which may explain its high discriminative value. $\mathrm{TT}_{\text {peak - to -peak is probably not affected at }}$ all which contributes to its highest rank as a discriminating parameter.

- three causes for overlap (or false classification) between the parameter ranges are identified when they are applied to three arteriographic groups. They comprise error or variation in the measurement, several physiological causes and finally the conceptual difference between a continuous variable (hemodynamic parameter) and a stratified discontinuous variable (arteriography).

- the Kappa-corrected diagnostic accuracy is proposed as a better measure for agreement between two methods than sensitivity or specificity, especially if more than two groups of disease are recognized.

-..- the performance of the CW and the multi-gate pulsed Doppler systems, measuring PI and DT for subsequent calculation of damping and TT in the femoro-popliteal area, is similar when the efficacy of these parameters for discrimination of three arteriographic groups is determined with discriminant analysis. The diagnostic accuracy with both Doppler systems is $80 \%$. This set of noninvasive hemodynamic parameters seems indeed sensitive enough, not only to classify significant atherosclerotic disease in the femoro-popliteal vascular segment, but also to follow in time its functional behaviour resulting from progression of disease and the subsequent response of the collateral system. 


\subsection{Future work}

When trying to relate the significance of this study to the literature on the subject, one cannot help but feell that more questions have been raised than answered.

The femoro-popliteal pathway was used as the 'testbed' for comparison of invasive and noninvasive parameters (arteriography and hemodynamic parameters) because they seem to be well controlled in this particular part of the vascular pathway.

The three comerstones of this type of study - the hemodynamic parameters, a 'gold-standard' for their verification and the statistical methods for their expression - come under considerable strain when examined in detail.

It has been suggested in the introduction to Chapter II that clinical vascular anatomy and pathophysiology have gone through successive stages of development, separated in time and by discipline. This has led to the present situation in which it is still unknown how to cope with arteriographic information in a meaningful and above all, reliable way. The awareness that single plane arteriography can be misleading is rapidly growing, which calls for converging diagnostic activities of the radiologist, the clinical physiologist and the surgeon in charge of the patient. The stage has been reached, it is believed, that the physiologist has managed to convince the clinician that physical laws govern the behaviour of pulsatile blood flow, so that the time has come for analytical investigations regarding the reliability of arteriography to diagnose disease and to discriminate between significant atherosclerotic lesions of increasing severity. There is need for an interobserver variability study on biplane arteriography, using intra-arterial pressure measurement as a new 'Gold standard'.

Doppler ultrasonic instruments provide versatile means for the noninvasive investigation of the vascular system, with future possibilities seemingly unlimited owing to the rapidly expanding microprocessor industry. However, a major area of study has been neglected in the past related to the beat-to-beat reproducibility of the Doppler signal and its derived hemodynamic parameters. It is obvious that many factors are simultaneously influencing the error or variation of the individual measurement. Biological (arrhythmia, respiration, old age, calcified arterial wall), technical (the choice of signal processing, the influence of signal-to-noise ratio), and hemodynamic (parabolic or plug flow velocity, blood pressure and compliance of the arterial wall, reflected pressure waves) causes of error or variation are identified, although their individual contribution cannot be quantified at present. As indicated in section 3.7.1., special studies, experimental as well as clinical, are needed under well controlled circumstances to learn more about these sources of error or variation. 
The clinical significance of many hemodynamic parameters, derived from the Doppler velocity wave form, has been analysed, because health or disease are accompanied by characteristic velocity patterns. The conceptual simplicity of PI and damping is appealing to clinicians but it cannot be denied that much subtle information, regarding the functioning of the vascular system, is discarded with their application. It is therefore believed that Fourier analysis of the velocity wave form, coming within reach of the practising clinician, will take the lead in this particular field of investigation, because there is increasing evidence that the wave form countour mirrors small changes in the physiological behaviour of the vascular system.

The pulse propagation time is a second parameter of considerable importance and it has been suggested in this study that transit-time, as measured on the systolic peak of the velocity wave form using the ECG as a fixed reference, is of

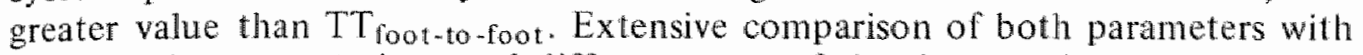
different instrumentations and different ways of signal processing, are necessary to clarify their position as important parameters for the detection of atherosclerotic disease.

This study has been exclusively concerned with a noninvasive method for the functional evaluation of the femoro-popliteal pathway. This arterial segment was chosen because of the good ultrasonic access offered by the arteries in the high femoral and popliteal areas. The results of this study may not be generalized and thus applied to other segments of the vascular system, without additional investigations, although no essentially different methods or techniques were used by the Guy's group in their evaluation of vascular segments above and below the femoropopliteal area, other than using a lower emission frequency in the aorto-iliac region.

Limitations of segmental disease detection are experienced when local disease, at the proximal or distal site of measurement, is the cause of disturbance of the flow velocity. PI measurements should be carried out as far away from significant lesions as possible to reduce the influence of reflections and turbulence. However, local disease may remain unnoticed by the examiner, especially when $\mathrm{CW}$ techniques are used. It is therefore believed that the techniques such as developed by Strand ness'group which are primarily concerned with the detection of local causes of flow disturbances, should be combined with segmental diagnostic methods. If the examiner has convinced himself that no significant disease is present at either site of measurement, he then may safely proceed to segmental parameter analysis.

That pulsed Doppler techniques may play an important part in these future studies seems evident as judged from the vast evolution of multi-gate pulsed Doppler instruments. The first generation instrument, of the type constructed in Maastricht and described in this study, seems to be an adequate tool for the analysis of the temporal and spatial behaviour of the velocity profile. With increasing resolution along the ultrasonic beam more will become known about the significance of local turbulence in the vicinity of wall irregularities previously undetectable with any technique.

Whether the spatial orientation offered by the multi-gate pulsed Doppier system is of equal satisfaction to the examiner as the B-mode imaging modality of the Duplex scanner, is a question of direct practical interest. The versatility of the information obtained with the multi-gate system is remarkable, as was illustrated 
in this study, and this quality is expected to improve with future generations of this instrument. B-mode imaging and the mono-gate pulsed Doppler system, with its known angle of insonation provided by the Duplex scanner, allow the examiner to sample velocities in a well controlled manner in both transverse and longitudinal directions of the artery, permitting repeated sampling at identical sites, which is important for follow-up studies. However, no information on the temporal behaviour of all velocity laminae is obtained. A comparison between both instruments for the follow-up of patients with atherosclerotic disease in the difficult diagnostic area of the common femoral and deep femoral area, would therefore be of great interest.

The abundance of data provided by noninvasive comparative studies necessitates computer-assisted techniques for administration, storage and analysis. Even more important are statistical techniques aiming at reduction of these vast quantities of data through elimination after analysis. Discriminant analysis, one of the many possible methods, was used in the present study to identify the best hemodynamic parameter (reduction) but also to grade or classify a vascular segment into an arteriographic group with the hemodynamic parameters at hand (classification). Refinement of these methods should be considered, because future comparative studies, especially if Fourier analysis is contemplated, will provide the investigator with massive digital information, unmanageable with traditional techniques.

The suggestion that improvement or deterioration of disease in a particular arterial segment might be 'monitored' on a discriminant analysis diagram should be further examined with clinical follow-up studies in unoperated patients, because the prospect of 'an early warning system' is highly desirable.

It is the opinion of the author that terms like specificity and sensitivity must disappear from the medical literature because they are grossly inadequate to describe the agreement present between two diagnostic techniques if more than two groups are recognized. Kappa was introduced in this study to correct for differences in the composition of patient groups so that the results from different studies could be compared. It is believed that refinement of this statistical method, e.g. with the introduction of weighting factors in order to recognize different degrees of false classifications, will increase its practical value. These complicated mathematical techniques call for active participation of mathematicians in this field of research.

A sensitive method aiming at quantification of arterial disease on a continuous scale can be developed only if the clinician is willing to cooperate with the basic scientist. A truly multidisciplinary approach is prerequisite for its success. 


\section{Summary}

This thesis reports on the practical value of a noninvasive method for the discrimination of three arteriographic groups in the femoro-popliteal pathway. Two Doppler instrumentations are compared, a commercially available continuouswave instrument (CW) and a multi-gate pulsed Doppler system (PD), both measuring the velocity at several arterial sites in the femoro-popliteal pathway with one transducer, for on-line calculation of the pulsatility index ( $\mathrm{PI}_{\mathrm{CW}}$ and $\mathrm{PI}_{\mathrm{PD}}$ ) as well as the delay-time $\left(D T_{C W}\right.$ and $\left.D T_{P D}\right)$, which is defined as the time-lag between the $\mathrm{R}$-wave of the ECG and the arrival of the systolic peak of the velocity signal. The zero-crosser output of each Doppler instrumentation was fed into an especially designed instrument, the wave form analyser, for on-line calculation of PI and DT peak to-peak. Mean values of the PI and the DT (20-30 heartbeats) are used to compute damping $\left(\Delta_{C W}\right.$ and $\left.\Delta_{P D}\right)$ and transit-time (TT with both instrumentations between the common femoral and popliteal arteries. An additional damping factor between the deep femoral and popliteal arteries could be calculated using the multi-gate pulsed Doppler system, because it allows for well-controlled centerstream velocity measurements at known distances away from the transducer e.g. the deep femoral artery. The multi-gate pulsed Doppler system displays simultaneously and instantaneously the temporal and spatial velocity behaviour in 32 adjacent sample volumes along the ultrasonic beam. The number of channels, actively engaged with velocity signals, can be counted, which provides an ultrasonic estimation of the arterial diameter. Subsequent analysis deals with the comparison and accuracy of these parameters to discriminate between three arteriographic groups.

The introduction pictures vascular surgery in its present day status and it emphasizes the need for practical, reliable and objective methods to assess function and loss of function in the vascular system.

Chapter 2 provides the reader with a condensed review of the literature on the physiology and patho-physiology of vascular disease. Several parameters of atherosclerotic disease are discussed. In the second part of this chapter attention is focussed on the physical properties of Doppler instruments and the versatility of their diagnostic application. The literature dealing with pulsatility index and transit-time is reviewed.

Chapter 3 accounts in detail for the materials and methods used in the study, placing some emphasis on the interpretation of arteriography and on the employed statistical method, discriminant analysis.

Several instrument-related and patient-examiner related sources of error and variation are recognized but only shortly discussed because extensive evaluation of their significance is considered to be beyond the scope and design of this study.

Eight sections in chapter 4 present the results of this study, each section followed by a concise discussion. 
Section 4.2: The mean value of PI and DT was determined over $20-30$ consecutive heartbeats and the corresponding parameters of both instrumentations, obtained at six arterial sites, were compared with linear regression and correlation analysis. $\mathrm{PIPD}$ and $\mathrm{PI}_{\mathrm{CW}}$ correlated poorly especially in the higher $\mathrm{PI}$ range, whereas $D_{P D}$ and $\mathrm{DT}_{\mathrm{CW}}$ showed acceptable correlation. Some of the possible explanations are discussed. The patient-and examiner-related sources of error or variation seem of less significance in the measurement of DT peak - to -peak than of PI.

Section 4.3: The impression existed during the preliminary phase of this study that the arterial diameter estimation with the multi-gate pulsed Doppler system corresponded well with the arterial diameter measured on the arteriogram. To evaluate the accuracy of these ultrasonically estimated diameters, they were correlated with the arteriographically measured diameter in six arterial sites. The best correlation was found in the superficial femoral artery (SFA) at $20 \mathrm{~cm}$ 's below the inguinal ligament $(R=0.8)$. The multi-gate pulsed Doppler system can accurately diagnose an occlusion in the proximal part of the SFA in $72-84 \%$. The conclusion that the ultrasonic estimation of the arterial diameter provides useful clinical evaluation is illustrated with many examples.

Section 4.4: The ankle systolic pressure index (ASPI) at rest and after exercise were evaluated in five groups of legs with various combinations of aorto-iliac and femoro-popliteal disease and in a group of legs free from atherosclerotic disease. The ability of the ASPI to distinguish normal legs from legs with significant atherosclerotic disease is extremely high (specificity 99\%), especially if the ankle pressure is determined after exercise. The cumulative effect of multi-level disease on the ASPI is evident, however, no deductions can be made regarding the localization of significant lesions.

Section 4.5: The influence of concomitant aorto-iliac disease on the value of damping $\left(\Delta_{C W}\right.$ and $\left.\Delta_{P D}\right)$, $\mathrm{TT}_{\text {peak -to-peak }}\left(\mathrm{TT}_{\mathrm{CW}}\right.$ and $\left.\mathrm{TT}_{\mathrm{PD}}\right)$ and the diameter of the SFA at 15 and $20 \mathrm{~cm}$ 's below the inguinal ligament is examined in five groups with various combinations of superficial femoral artery and aorto-iliac disease. No statistically significant differences between the mean values of these parameters in arteriographic groups with or without aorto-iliac disease are present, thus illustrating their independence from disease proximal to the femoral artery.

Section 4.6: If reflections occur in the femoral artery in the presence of significant atherosclerotic disease of the SFA, they may influence the contour of the velocity wave form and thus the value of PI. Thirty-eight percent of the common femoral artery velocity wave forms obtained with the pulsed Doppler system showed a dicrotic 'notch'. The dicrotic "notch' probably indicates the presence of reflected velocity waves, because they occur predominantly in association with occlusions in the proximal part of the SFA. It is likely that reflections in this area contribute to the overlap between the various parameters for different arteriographic groups.

Section 4.7: This section should be considered preparatory to section 4.9 since it deals with the previously described parameters in relation to 21 arteriographic subgroups composed by various combinations of significant lesions in the femoropopliteal pathway. It emphasizes the variability of atherosclerotic disease in this area. However, separate evaluation of each of these (small) groups was not considered useful so that each leg was re-allocated into one of three possible groups $(0=$ normal, $1=$ stenosis, 2 =occlusion). None of the hemodynamic parameters 
could individually distinguish between them because of mutual overlap between the three groups.

Section 4.8: The pulse wave velocity is known to be influenced by the intraluminal arterial pressure, so that the discriminative value of $\mathrm{TT}_{\text {peak-to-peak }}$ for femoro-popliteal disease might benefit from normalization for the blood pressure distal to a significant aorto-iliac lesion. $\mathrm{TT}_{\text {peak-to-peak in } 97 \text { normal legs was }}$ correlated with the systolic arterial pressure. Although a slight decrease of TT could be observed with increasing blood pressure, the measurement error or variation, represented by the scatter around the regression line, would certainly obscure the possible benefit of normalization.

Section 4.9: Since none of the hemodynamic parameters could individually classify a leg into a particular arteriographic group, discriminant analysis was employed to make optimal use of the variance present over all parameters. Three arteriographic groups were à priori defined and the diagnostic accuracy of several parameter combinations was à posteriori determined after calculation of two linear functions. This was done separately for parameter combinations obtained with both instrumentations. It appeared that only marginal differences were present between the diagnostic accuracy of corresponding parameter combinations (approx. 80\%). Transit-time ( $\mathrm{TT}_{\mathrm{CW}}$ and $\mathrm{TT}_{\mathrm{PD}}$ ) was the best discriminatory parameter followed by damping. Of interest was that damping between the deep femoral and the popliteal artery ( $\Delta$ prof-poppD $)$ was a better discriminator than damping between the common femoral and the popliteal artery ( $\Delta$ com-poppo). To replace terms like sensitivity and specificity, Kappa is proposed as a better measure for pairwise agreement when dealing with more than two groups of disease not only because it corrects for differently composed patient populations but also because it can express the meaning of different 'degrees' of false positive or false negative ratings.

One example of discriminant analysis is thoroughly discussed, to outline the practical usefulness of the 'butterfly' shaped diagram presented with this type of analysis. It is suggested that the hemodynamic parameters, as well as the applied analysis technique, are sensitive enough to visualize differences in collateral function of individual patients.

In chapter five a discussion of the most prominent findings of chapter four is presented. Eight major conclusions are summarized regarding:

- the alleged inferiority of a zero-crossing meter for quantitating measurements using PI and DT, calculated from the analog wave form

- the on-line measurement with one transducer and calculation of damping and $\mathrm{TT}_{\text {peak -to-peak }}$ using averaged (20-30 heartbeats) values of PI and DT

- the anatomic information supplied by the multi-gate pulsed Doppler system

- the absence of improvement of TT peak.to-peak following "normalization" for systolic blood pressure

- the influence of reflected velocity waves on the various hemodynamic parameters

- the cause of overlapping parameter ranges

- the desirability for the introduction of a better measure for pair-wise agreement (Kappa)

- the similar performance of the CW and the multi-gate pulsed Doppler system to classify atherosclerotic lesions in the femoro-popliteal pathway with the hemodynamic parameters damping and TT, according to three arteriographic groups. 


\section{Samenvatting}

Dit proefschrift beschrijft de bruikbaarheid van een non-invasieve methode ter onderscheiding van atherosclerotische afwijkingen in het femoro-popliteale traject die zijn ingedeeld in drie arteriografische groepen. Twee Doppler instrumentaties worden vergeleken, nl. een commercieel verkrijgbaar continuous wave instrument (CW) en een multi-gate pulsed Doppler systeem (PD). Aan het snelheidssignaal werd de pulsatiliteitsindex $\left(\mathrm{PI}_{C W}\right.$ en $\left.\mathrm{PI}_{\mathrm{PD}}\right)$ bepaald, die is gedefinieerd als het verschil van de maximale en minimale snelheden gedeeld door de gemiddelde snelheid tijdens één hartcyclus. Tevens werd de vertragingstijd (DT peak-to-peak) tussen de R-golf van het ECG en de aankomst van de snelheidsgolf bij de meetplaats (DT $T_{C W}$ en $D T_{P D}$ ) geregistreerd. De metingen werden op meerdere plaatsen in het femoro-popliteale traject uitgevoerd met slechts één transducer.

Het uitgangsvoltage van de nulpuntenteller, gebruikt in beide apparaten, werd naar een speciaal ontworpen instrument geleid, de 'wave form analyser', woor automatische bepaling van beide parameters. Gemiddelde waarden van PI en DT peak-10-peak (20-30 hartslagen), met beide instrumentaties verkregen, werden gebruikt om de dampingfactor (PI distaal/PI proximaal) en de looptijd (DT distaal-DT proximaal) te berekenen over het arteriële traject tussen de arteria femoralis communis en de arteria poplitea.

Het multi-gate pulsed Doppler-systeem biedt de mogelijkheid om een tweede dampingfactor te berekenen over dit traject omdat snelheidsinformatie verkregen kan worden vanuit het midden van een arterie gelegen op bekende afstand van de transducer, zoals b.v. in de arteria profunda femoris. De bloedstroomsnelheid in 32 naast elkaar gelegen bemonsteringseenheden in de richting van de ultrageluidsbundel, kan door het multi-gate pulsed Doppler systeem tegelijkertijd zichtbaar worden gemaakt. Het aantal kanalen waarin snelheidssignalen aanwezig waren, gaf een ultrasonore schatting van de arteriële diameter. Dit proefschrift heeft de vergelijking en betrouwbaarheidsbepaling van genoemde parameters (damping en looptijd) om drie arteriografische groepen van elkaar te onderscheiden, tot onderwerp.

De introductie schetst de behoefte van de hedendaagse vaatchirurgie aan een praktische, betrouwbare en objectieve methode om functie en verlies van functie van bloedvaten te meten.

In hoofdstuk 2 wordt een beknopt overzicht gegeven van de literatuur over de fysiologie en pathofysiologie van atherosclerotische bloedvaten. Verschillende diagnostische parameters worden behandeld. In het tweede deel van dit hoofdstuk is de aandacht meer gericht op de fysische eigenschappen van Doppler instrumenten en op de veelzijdigheid van hun diagnostische toepassingen. Een overzicht wordt gegeven van de literatuur die betrekking heeft op PI en looptijd.

Hoofdstuk 3 behandelt in detail het materiaal en de methodiek zoals ze in deze studie werden gebruikt. De beoordeling van arteriografie krijgt hierbij uitvoerig 
aandacht, evenals de gebruikte statistische methode, discriminant analyse. Verschillende oorzaken voor het optreden van fouten en variaties in de meetwaarden worden genoemd en kort besproken, omdat een uitgebreide behandeling buiten het bestek van dit proefschrift valt.

Secties $4.2 \mathrm{t} / \mathrm{m} 4.9$ van hoofdstuk 4 geven de resultaten van deze studie, elk deel wordt gevolgd door een beknopte discussie.

Sectie 4.2: Na berekening van de gemiddelde PI- en DT-waarden over 20-30 opeenvolgende hartslagen, werden de overeenkomende parameters, zoals deze met beide instrumentaties op zes verschillende plaatsen in het bovenbeen waren gemeten, vergeleken door middel van correlatie- en lineaire regressieanalyse.

$\mathrm{PI}_{P D}$ en $\mathrm{PI}_{C W}$ correleren slecht, vooral bij hogere waarden van $\mathrm{PI}$, terwijl TTPD en $\mathrm{TT}_{\mathrm{CW}}$ redelijk goed met elkaar overeen komen. Een korte discussie wordt gewijd aan enkele mogelijke oorzaken. Oorzaken van meetfouten of variaties die terug te voeren zijn op de patient of de onderzoeker worden van minder belang geacht bij de bepaling van $\mathrm{DT}_{\text {peak -to-peak }}$ dan bij PI.

Sectie 4.3: In een vroeg stadium van deze studie werd reeds de indruk verkregen dat de ultrasonore schatting van de arteriële diameter met behulp van het multigate pulsed Doppler apparaat goed overeenkwam met de arteriografische diameter van een arterie. De kwaliteit van deze vermeende correlatie werd getoetst op zes plaatsen in het bovenbeen.

De beste correlatie bestaat in de arteria femoralis superficialis (AFS) gemeten $20 \mathrm{~cm}$ onder het ligament van Poupart $(\mathrm{R}=0.8)$. Het multi-gate pulsed Doppler systeem blijkt een afsluiting in het proximale deel van de AFS met een betrouwbaarheid van 72 tot $84 \%$ te kunnen bepalen. De conclusie dat de ultrasonore schatting van de arteriële diameter nuttige informatie oplevert voor de klinicus, wordt met vele voorbeelden geillustreerd.

Sectie 4.4: De waarde van systolische bloeddrukmetingen ter hoogte van de enkel in rust en na inspanning op een lopende band, werd onderzocht in vijf groepen benen met verschillende combinaties van aorto-iliacale en femoro-popliteale afwijkingen, èn in een groep benen zonder arteriële afwijkingen.

De diagnostische betrouwbaarheid van de arm-enkel index, om normale benen te onderscheiden van benen met significante arterielle afwijkingen, is zeer hoog (specificiteit $99 \%$ ), vooral als de index is bepaald na inspanning. Het cumulatieve effect van atherosclerotische laesies of meerdere niveaus op de enkel-arm index is duidelijk, ofschoon het geen ruimte laat voor conclusies betreffende hun localisatie.

Sectie 4.5: De invloed van aorto-iliacale afwijkingen op damping, $\mathrm{TT}_{\text {peak-to-peak }}$ en de diameter van de AFS op 15 en $20 \mathrm{~cm}$ onder het ligament van Poupart werd onderzocht in vijf verschillende groepen benen.

Er bestaan geen statistisch significante verschillen tussen de gemiddelde waarden wan de parameters afkomstig van benen met en zonder aorto-iliacale afwijkingen, hetgeen de onafhankelijkheid van deze parameters, voor afwijkingen boven het femoro-popliteale traject, bevestigt.

Sectie 4.6: Als tengevolge van atherosclerotische afwijkingen van de AFS reflecties voorkomen in de arteria femoralis communis, dan zouden deze de vorm van de snelheidscurve kunnen beïnvloeden en dientengevolge de waarde van PI.

In $38 \%$ van de benen met een afgesloten $\mathrm{AFS}$, blijkt een zogemaande 'dicrotic 
notch" aanwezig te zijn in de snelheidscurve zoals deze over de arteria femoralis communis was verkregen met het multi-gate pulsed Doppler systeem. De aanwezigheid van een 'dicrotic notch' duidt op het bestaan van gereflecteerde snelheidsgolven, omdat deze kenmerkende vervorming van de snelheidscurve vooral wordt gezien in samenhang met afsluitingen in het proximale gedeelte van de $\mathrm{AFS}$. Het is niet onwarschijnlijk dat reflecties in dit gebied bijdragen tot de overlapping van het bereik van de parameters tussen de verschillende arteriografische groepen.

Sectie 4.7: Deze sectie dient te worden beschouwd als de aanloop tot sectie 4.9. De eerder beschreven parameters werden vergeleken aan de hand van 21 verschillende arteriografische subgroepen, die werden samengesteld uit combinaties van significante laesies in het femoro-popliteale traject.

De variabiliteit van atherosclerotische afwijkingen in dit gebied komt hierin duidelijk tot uiting. De benen worden heringedeeld in drie groepen $(0=$ normaal 1 = stenose, 2 = occlusie), omdat apart onderzoek van de meeste (kleine) groepen om statistische redenen niet zinvol wordt geacht. Niet één van de haemodynamische parameters blijkt in staat om een been met zekerheid in één van deze drie groepen in te delen tengevolge wan de bestaande overlapping.

Sectie 4.8: De intra-arteriële bloeddruk beinvloedt de snelheid van de polsgolf,

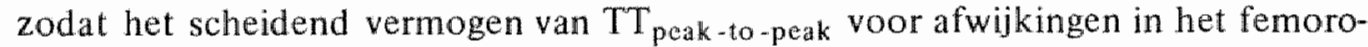
popliteale traject mogelijk verbetert na correctie (normalisatie) voor de heersende bloeddruk, distaal wan een atherosclerotische laesie. $\mathrm{TT}_{\text {peak-to-peak werd gecorre- }}$ leerd met de systolische arteriële bloeddruk in 97 normale benen.

Ofschoon een mogelijke afname van de looptijd met toenemende bloeddruk kan worden vastgesteld, blijken, zoals duidelijk werd uit de spreiding van de waarden rond de regressielijn, meetfouten of variaties het mogelijk goede effect hiervan te verhullen.

Sectie 4.9: Geen van de haemodynamische parameters bleek in staat een been met zekerheid in een bepaalde arteriografische groep in te delen. Om deze reden werd gebruik gemaakt van discriminant analyse, zodat de bestaande variantie over de parameters optimaal kon worden benut. De diagnostische betrouwbaarheid van de verschillende parametercombinaties, met het doel de benen in te delen (à posteriori) nat hun arteriografische classificatie (à priori), werd bepald na berekening van twee lineaire functies. Deze analyse werd afzonderlijk uitgevoerd op parameter combinaties verkregen met beide instrumentaties.

Er blijken slechts marginale verschillen aanwezig te zijn tussen de diagnostische betrouwbarheid van corresponderende parametercombinaties afkomstig van beide instrumentaties (ongeveer $80 \%$ ). Looptijd blijkt de hoogste discriminerende warde te hebben, gevolgd door damping.

Opmerkelijk is dat damping ( $\Delta$ prof-poppD), berekend over de arteria profunda femoris en de arteria poplitea, beter discrimineert tussen de drie arteriografische groepen dan damping ( $\Delta$ com-poppo $)$ over de arteria femoralis communis en de arteria poplitea.

Ter vervanging van de begrippen 'sensitivity' en 'specificity' wordt Kappa geintroduceerd als en betere maat voor overeenstemming tussen twee methodes, omdat het de mogelijkheid biedt deze diagnostische overeenstemming (diagnostic accuracy) te corrigeren voor verschillend samengestelde groepen van benen. Tevens biedt Kappa de mogelijkheid om verschillende nuances van 'fout' (vals positief of vals negatief) tot uitdrukking te brengen. 
Eén voorbeeld van discriminant analyse wordt uitvoerig besproken teneinde de praktische bruikbaarheid van de door de analyse verkregen 'vlinderfiguur' duidelijk naar voren te brengen.

De indruk bestaat dat zowel de haemodynamische parameters als de toegepaste analyse-methode gevoelig genoeg zijn, om het functieverschil tussen collateraalsystemen van individuele patienten zichtbaar te maken.

In hoofdstuk 5 worden de belangrijkste bevindingen, zoals gepresenteerd in hoofdstuk 4, nader besproken. De acht voornaamste conclusies worden hieronder kort weergegeven. Ze hebben betrekking op:

- het vermeende slecht functioneren van een nulpuntenteller bij de berekening van PI en DT aan het analoge snelheidssignaal.

- de effectiviteit van de automatische PI en DT bepaling met één transducer en de hieropvolgende berekening van damping en TT met de gemiddelde parameter waarden (20-30 hartslagen).

- de anatomische informatie die wordt verkregen door toepassing van het multi-gate pulsed Doppler systeem.

- de uitgebleven verbetering van de discriminerende waarde van $\mathrm{TT}_{\text {peak-to-peak }}$ na normalisatie voor de systolische bloeddruk.

- de invloed van gereflecteerde snelheidsgolven op betrouwbaarheid van de onderzochte haemodynamische parameters.

- het complex van oorzaken die bijdragen tot de geconstateerde overlapping van de parameters tussen de arteriografische groepen.

- de wenselijkheid om een betere maat voor overeenstemming tussen twee methodes in te voeren (Kappa).

- de gelijkheid van het CW en het multi-gate pulsed Doppler systeem wat betreft hun vermogen om afwijkingen in het femoro-popliteale traject met behulp van damping en looptijd in te delen overeenkomstig drie arteriografische groepen. 


\section{Appendix}

Table I The differences between the mean parameter value (each parameter indicated with its abbreviation) of six arteriographic groups (table 4.3 ), were statistically tested with the MannWhitney test (section 4.5). The difference was considered significant (s.) if $P<0.001$ (2-tailed) and not significant (n.s.) if $\mathrm{P}>0.001$ (2-tailed). The question mark (?) indicates an inconclusive result. The number of legs (N) is given per group.

\section{$\triangle$ prof-popPD}

\begin{tabular}{lrrrrrr}
$\mathrm{N}=$ & 98 & 19 & 7 & 18 & 27 & 49 \\
& 0 & 1 & 2 & 3 & 4 & 5 \\
0 & $\mathrm{X}$ & $n . s$. & s. & s. & s. & s. \\
1 & & $\mathrm{X}$ & s. & s. & n.s. & s. \\
2 & & & $\mathrm{X}$ & n.s. & n.s. & n.s. \\
3 & & & & $\mathrm{X}$ & s. & n.s. \\
4 & & & & & $\mathrm{X}$ & s. \\
5 & & & & & & $\mathrm{X}$ \\
\hline
\end{tabular}

\section{$\Delta$ com-pop ${ }_{\mathrm{CW}}$}

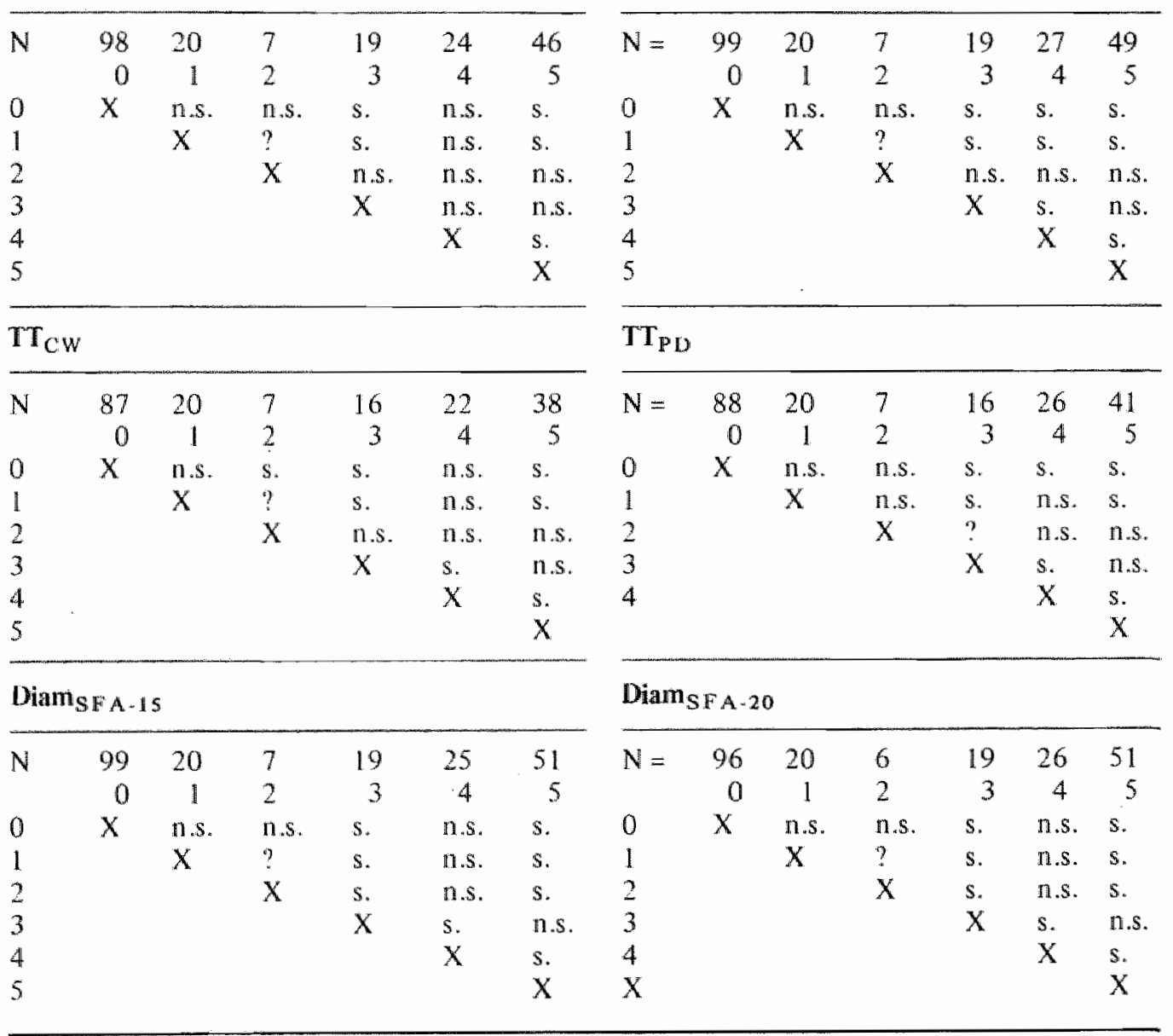


Table II The differences between the mean parameter value (each parameter indicated with its abbreviation) of three arteriographic groups (table 4.6 ), were statistically tested with the Mann-Whitney test (section 4.7). The difference was considered significant (s.) if $P<0.001$ (2-tailed) and not significant (n.s.) if $P>0.001$ (2-tailed). The number of legs $(\mathrm{N})$ is given per group.

\begin{tabular}{lrrr}
\multicolumn{4}{l}{$\Delta$ com-pop } \\
$\mathrm{N}=$ & 123 & 36 & 71 \\
& 0 & 1 & 2 \\
0 & $\mathrm{X}$ & $\mathrm{s}$. & $\bar{s}$. \\
1 & & $\mathrm{X}$ & $\mathrm{s}$. \\
2 & & & $\mathrm{X}$
\end{tabular}

$\begin{array}{lrrr}\Delta \text { com-popPD } \\ \mathrm{N}= & 124 & 38 & 74 \\ & 0 & 1 & 2 \\ 0 & \mathrm{X} & \mathrm{s.} & \mathrm{s} . \\ \mathrm{l} & & \mathrm{X} & \mathrm{s} . \\ 2 & & & \mathrm{X}\end{array}$

\section{$\Delta$ prof-popPD}

\begin{tabular}{rrrr}
\hline $\mathrm{N}=$ & 124 & 38 & 73 \\
& 0 & 1 & 2 \\
0 & $\mathrm{X}$ & $\mathrm{s}$. & $\mathrm{s}$. \\
1 & & $\mathrm{X}$ & $\mathrm{s}$. \\
2 & & & $\mathrm{X}$ \\
\hline
\end{tabular}

\begin{tabular}{lrrr}
$\mathrm{TT}_{\mathrm{PD}}$ & & & \\
\hline $\mathrm{N}=$ & 113 & 35 & 62 \\
& 0 & 1 & 2 \\
0 & $\mathrm{X}$ & $\mathrm{s}$. & $\mathrm{s.}$ \\
1 & & $\mathrm{X}$ & $\mathrm{s.}$ \\
2 & & & $\mathrm{X}$ \\
\hline
\end{tabular}

\begin{tabular}{lrrr}
$\mathrm{TT}_{\mathrm{CW}}$ & & \\
\hline $\mathrm{N}=$ & 112 & 32 & 59 \\
& 0 & 1 & 2 \\
0 & $\mathrm{X}$ & $\mathrm{s}_{*}$ & $\mathrm{~s}$. \\
1 & & $\mathrm{X}$ & $\mathrm{s}$ \\
2 & & & $\mathrm{X}$ \\
\hline
\end{tabular}

\begin{tabular}{lrrr}
\multicolumn{2}{l}{ Diam $_{\text {pop }}$} & & \\
\hline $\mathrm{N}=$ & 128 & 38 & 77 \\
& 0 & 1 & 2 \\
0 & $\mathrm{X}$ & s. & s. \\
1 & & $\mathrm{X}$ & n.s. \\
2 & & & $\mathrm{X}$ \\
\hline
\end{tabular}

\begin{tabular}{lrrr} 
Diam $_{\text {SFA.20 }}$ & \\
\hline $\mathrm{N}=$ & 124 & 38 & 77 \\
& 0 & 1 & 2 \\
0 & $\mathrm{X}$ & $\mathrm{s.}$ & $\mathrm{s.}$ \\
1 & & $\mathrm{X}$ & $\mathrm{n.s.}$ \\
2 & & & $\mathrm{X}$
\end{tabular}


Table III The conversion of a three-way table (table 4.8) irto three two-way tables (I, II, III) is depicted and the statistical terms (false positive rate, false negative rate, specificity, sensitivity) are calculated for each two-way table.

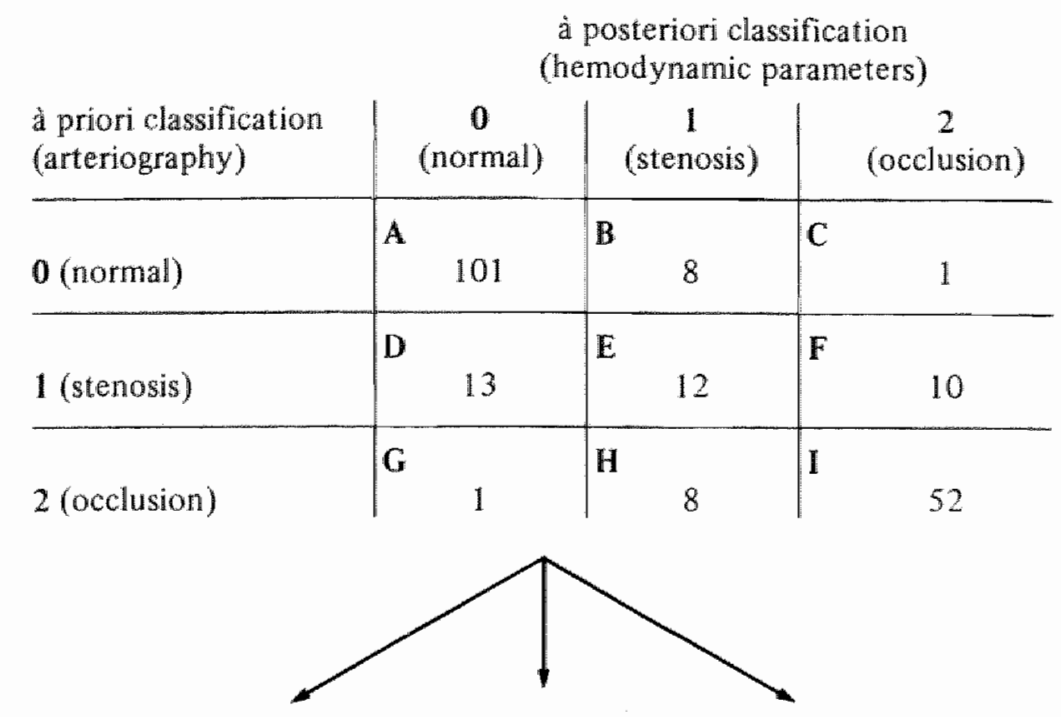

II

III

\begin{tabular}{c|c|c|c|c|c|c|c|c} 
& 0 & 1,2 & & 1 & 0,2 & & 2 & 0,1 \\
\hline 0 & A & BC & 1 & E & DF & 2 & I & GH \\
\hline 1.2 & DG & EFHI & 0.2 & BH & ACGI & 0.1 & CF & ABDE
\end{tabular}

false pos. : $\frac{\mathrm{BC}}{\mathrm{BCEFHI}}=9.8 \%$ false pos. : $\frac{\mathrm{DF}}{\mathrm{DFACGI}}=12.9 \%$ false pos. : $\frac{\mathrm{GH}}{\mathrm{GHABDE}}=6.2 \%$ false neg. : $\frac{\mathrm{DG}}{\mathrm{ADG}}=12.1 \%$ false neg. $: \frac{\mathrm{BH}}{\mathrm{EBH}}=57.1 \%$ false neg. $: \frac{\mathrm{CF}}{\mathrm{ICF}} \quad=17.4 \%$ specificity: $\frac{\mathrm{A}}{\mathrm{ABC}}=91.8 \%$ specificity: $\frac{\mathrm{E}}{\mathrm{EDF}} \quad=32.4 \%$ specificity: $\frac{1}{\mathrm{IGH}} \quad=85.2 \%$ sensitivity: $\frac{\mathrm{EFHI}}{\mathrm{DGEFHI}}=85.4 \%$ sensitivity: $\frac{\mathrm{ACGI}}{\mathrm{BHACGI}}=90.6 \%$ sensitivity: $\frac{\mathrm{ABDE}}{\mathrm{CFABDE}}=92.4$ 


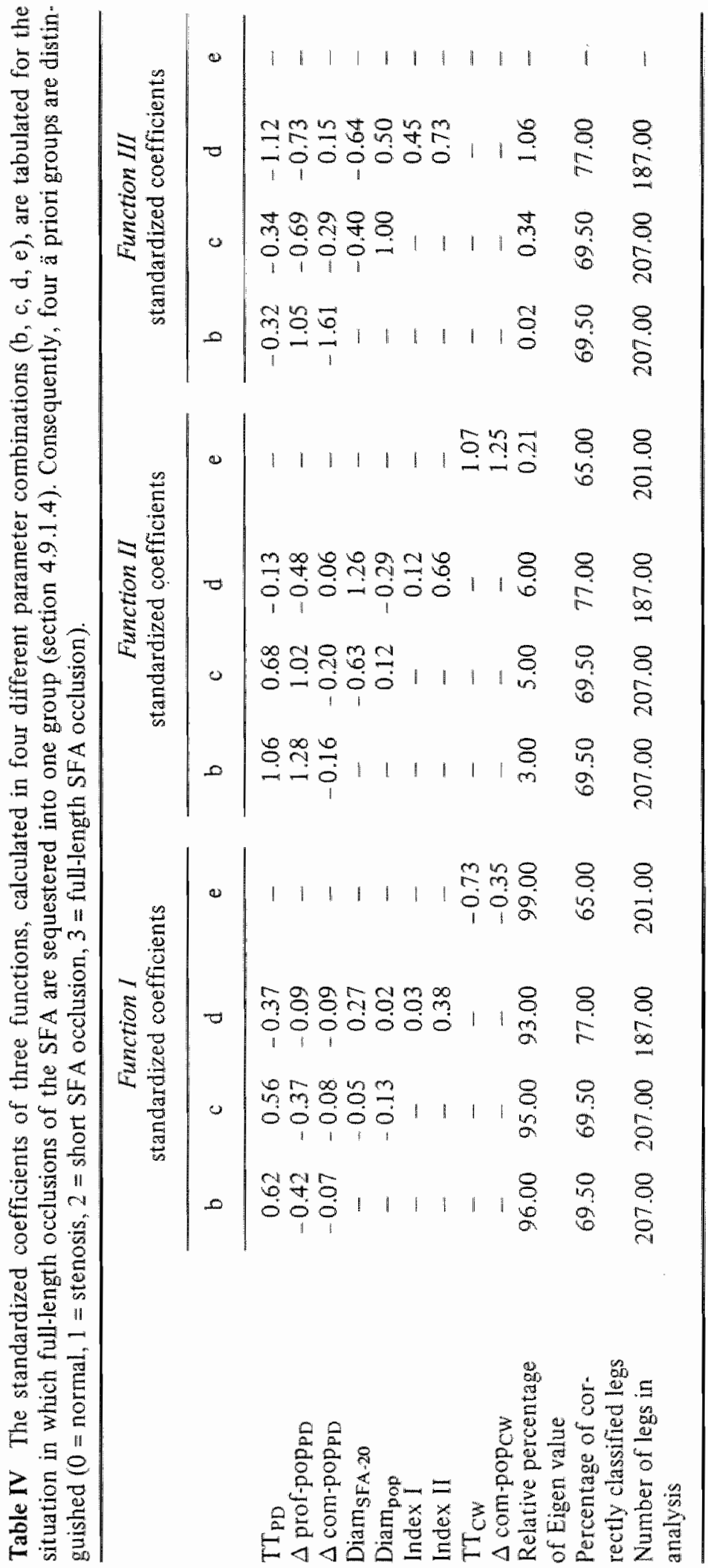




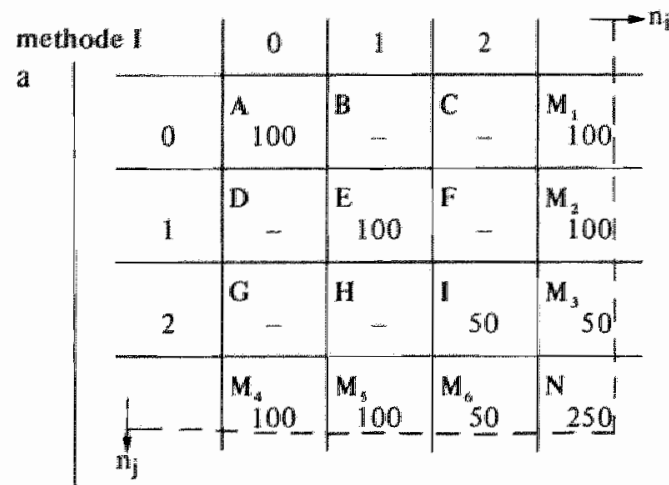

b

\begin{tabular}{c|c|c|c|c} 
& 0 & 1 & 2 & \\
\hline 0 & 100 & & & 100 \\
\hline 1 & & 30 & & 30 \\
\hline 2 & 70 & & 50 & 120 \\
\hline & 170 & 30 & 50 & 250
\end{tabular}

$\mathrm{c}$

\begin{tabular}{c|c|c|c|c} 
& 0 & 1 & 2 & \\
\hline 0 & 100 & & & 100 \\
\hline 1 & & 30 & & 30 \\
\hline 2 & & -70 & 50 & 120 \\
\hline & 100 & 100 & 50 & 250
\end{tabular}

d

\begin{tabular}{c|c|c|c|c} 
& 0 & 1 & 2 & \\
\hline 0 & 130 & & & 130 \\
\hline 1 & & 30 & & 30 \\
\hline 2 & & 70 & 20 & 90 \\
\hline & 130 & 100 & 20 & 250
\end{tabular}

e

\begin{tabular}{c|c|c|c|c} 
& 0 & 1 & 2 & \\
\hline 0 & 150 & & & 150 \\
\hline 1 & & 10 & & 10 \\
\hline 2 & & 70 & 20 & 90 \\
\hline & 150 & 80 & 20 & 250
\end{tabular}

Kappa $=\frac{P_{b}-P_{c}}{1-P_{C}}$

dagniostic accuracy $P_{o}=\frac{A+E+1}{250}=1.0$.

$P_{c}=\left(\frac{M_{1}}{N} \times \frac{M_{4}}{N}\right)+\left(\frac{M_{2}}{N} \times \frac{M_{3}}{N}\right)+\left(\frac{M_{3}}{N} \times \frac{M_{6}}{N}\right)+\cdots$

$\cdots+\left(\frac{\mathrm{M}_{\mathrm{ni}_{\mathrm{i}}}}{\mathrm{N}} \times \frac{\mathrm{M}_{\mathrm{nj}}}{\mathrm{N}}\right)=0.36$

$K=\frac{1-0.36}{1-0.36}=1.0$

$P_{0}=0,72$

$P_{c}=\left(\frac{100}{250} \times \frac{170}{250}\right)+\left(\frac{30}{250} \times \frac{30}{250}\right)+\left(\frac{120}{250} \times \frac{50}{250}\right)=0,382$

Kappa (- corrected diagnostic accuracy): 0,54

$P_{0}=0,72$

$P_{c}=\left(\frac{100}{250} \times \frac{100}{250}\right)+\left(\frac{30}{250} \times \frac{100}{250}\right)+\left(\frac{120}{250} \times \frac{50}{250}\right)=0,304$

Kappa ( - corrected diagnostic accuracy) :0,59

$\mathbf{P}_{0}=0,72$

$P_{c}=\left(\frac{130}{250} \times \frac{130}{250}\right)+\left(\frac{30}{250} \times \frac{100}{250}\right)+\left(\frac{90}{250} \times \frac{20}{250}\right)=0,346$

Kappa ( - corrected diagnostic accuracy): 0,57

$P_{0}=0,72$

$P_{c}=\left(\frac{150}{250} \times \frac{150}{250}\right)+\left(\frac{10}{250} \times \frac{80}{250}\right)+\left(\frac{90}{250} \times \frac{20}{250}\right)=0,40$

Kappa ( corrected diagnostic accuracy) $=0,53$ 
Table $V$ The method, Kappa, has been originally developed to study agreement between two or more independent observers using categorical data (yes or no) vaia nomimal scales $(0,1,2$ ....n). Kappa is the measure for pairwise agreement between both observers (or diagnostic methods) after chance agreement has been removed. The mechanism is best understood with an exercise as given in this table.

Suppose that two diagnostic methods (I and II) are compared in a population of N (250) patients. The agreement (or diagnostic accuracy of method II if method I is considered the "gold-standard'y between both methods is given by the diagonal cells of the three-way table divided by the total number of subjects under investigation $\left(\frac{A+E+1}{N}\right)$. In any $n$-way table (n $>2$ ) different "degrees" of false positive or false negative ratings are possible. Cells $\mathrm{C}$ and $\mathrm{G}$ are "more" falsely classified than cells $B$ and $H$ in the given examples. The possible number of different "grades' false classifications is determined by the discrimination into $\mathrm{n}$ scales offered by both methods. The number of different 'grades" false classifications will be $n-1$. The further away from the diagonal, the worse the agreement between method I and method II.

Example a shows perfect agreement so that the diagnostic accuracy is 1.0 with a maximum value of Kappa (1.0). Examples $b$ and $c$ with a diagnostic accuracy of 0.72 have 70 false classifications, but differently placed in the three-way table. If they are moved from cell $\mathrm{G}$ to cell $\mathrm{H}$, Kappa will increase from 0.54 to 0.59 , thus expressing the improvement in the "grade" of false classification. The diagnostic accuracy remains uninfluenced.

Examples $d$ and e illustrate the influence of one large group in which agreement is easy, e.g. normal subjects. The 70 false ratings in a decreasing population of diseased legs obviously have significance for the reliability of method. II. Kappa will decrease correspondingly while the value of the diagnostic accuracy remains unchanged.

Further reading on this subject:

Cohen J. Weighted Kappa, Psy chological bulletin 70, 213-220, 1968.

Fleiss J.L.: Measuring nominal scale agreement among many raters, Psychologicall bulletin 76 , $378 \cdot 382,1971$.

Light R.J.: Measures of response agreement for qualitative data, Psychological bulletin 76 , $365-377,1971$.

Koran L.M.: The reliability of clinical methods, data and judgments (I, II). N. Engl. J. Med. $642-646,695-701,1975$. 


\section{References}

1. Abramowiz 1.: The assessment of peripheral vascular insufficiency with the Doppler flowmeter. S. Afr. Med. J. $51: 67-70,1977$.

2. Abrechtgen D., Hauss $G$ : Femoropopliteall saphenous vein bypass for ocolusive atherosclerosis causing leg ischamia. Scand. J. Thor. Cardiovasc. Surg. 9: 48.55, 1975.

3. Allan $J$ S. Terry H.J.: The evaluation of an ultrasonic flow detector for the assessment of peripheral vascular disease. Cardionasc. Res. 3: 503-509, 1969.

4. Angelides N.S., Nicolaides A.N., Eastcott H.H.G.: The value of Doppler velocity tracings in femoropopliteal disease. In: Utraschall-Doppler-Diagnostik in der Angiologie. Kriessmann A. und Bollinger A. Thieme Verlag, Stutegart 1979.

5. Angelsen B.A.J., Brubakk A.O: Transcutaneous measurement of blood flow welocity in the human aorta. Cardiowosc. Res. $10: 368-379,1976$.

6. Arts M.G.J. and Roewros J.M.J.G.: On the instantaneous measurement of blood flow by ultrasonic means. Med, Biol. Eng. 10: 23-34, 1972.

7. Baird R.N., Bird D.R., Cliford P.C., Lusby R.J., Skidmore R., Woodcock I.P.: Upstream stenosis. Its diagnosis by Doppler signals from the femoral artery. Alrch. Surg. 115:1316-1322, 1980.

8. Baird R.N., Lusby R.J., Burd D.R., Giddings A.E.B., Skidmore R., Woodcock I.P., Horton R.E., Peacock J.H.: Pulsed Doppler angiography in lower

9. Baker D.W., Daigle, R.E, Noninvasive ultrasonic flowmetry. In: Cardionascular flow dynamics and medswements, Ed. Hwang and Normann, Park Press, Baltimore 1977.

10. Balker D.W., Strandness D.F., Johnson S.L.: Pulsed Doppler techniques : some examples from the university of Washington. Ultrasound Med. Biol, 2: 251-262, 1976.

11. Barendsen G.J.: Plethysmography. In: Methods in angiology, Ed.Verstraete. Martinus Nijhoff, The Hague 1980 .

12. Barner H.B., Kaminski D.L., Codd J.E, Kaiser G.C, Wilman V.L.: Hemodynames of autogenous femoropopliteal bypass. Arch. Surg. 109: 291-293, 1974.

13. Baskett J.J., Beastley M.G., Murphy G.J., Hyams D.E., Gosling R.G.: Screening for carotid junction disease by spectral analysis of Doppler signals. Cardiovasc. Res. 11: 147-155, 1977.

14. Beales J.S.M., Adcock F.A., Frawley J.E., Nathan B.E., McLachlan M.S.F., Martin P., Steiner R.E.: The adiological assessment of disease of the profunda femoris artery. Br. J. Radiol. 44: 854-859, 1971.

15. Bell G., Nielsen P.E., Wolfson B., Ulrich J., Engell H.C., Lassen N.A.: Measurements of systolic pressure in the limbs of patients with arterial occlusive disease. Surg. Gynecol. Obstet. 136: 177-181, 1973.

16. Bernhard V.M., Ray L.1., Militello J.P." The role of angioplasty of the profunda femoris artery in Levascularization of the ischemic limb. Surg. Gymecol Obstet. 142: 840-844, 1976.

17. Berguer R. Hwang N.H.C.; Critical arterial stenosis: a theoretical and experimental solution. Ann. Surg. $180: 39.50,1974$.

18. Berguer R., Cotton L.T., Sabri S.: Extended deep femoral angioplasty. Br. Med. J. 1: 469.471, 1973.

19. Bhackshear W.M., Phillips D.J., Chikos P.M., Ha,rley J.D., Thiele B.L., Strandness D.E.: Carotid artery velocity patterns in normall and stenotic vessels. Stroke 11:67-71, 1980.

20. Blackshear W.M., Phillips D.J., Thiele B.L., Hirsch J.H., Chikos P.M., Marinelli M.R., Ward K.J., Strandness D.E. Detection of carotal occlusive diseage by ultranic imaging and pulsed Doppler spectrum analysis. Surgery 86:698-706, 1979 .

21. Blackshear W.M. Phillips D.J., Strandness D.E.: Pulsed Dopplet assessment of nommal human femoral attery velocity patterns. J. Surg. Res: 27: 73-83, 1979.

22. Bloor K.: Natural history of arteriosclerosis of the lower extremities. Ann. Roy. Coll. Surg. Engl. 28: $36-52,1960$.

23. Bone G.E. Hayes A.C., Slaymaker E., Barnes R.W.: Value of segmental bone blood pressures in predicting results of aortofomoral bypass. Am. J. Surg. 132:733m $738,1976$.

24. Bouhoutsos $J$. Martin $P$. The influence of age on prognosis after arterial surgery for atherosclerosis of the lower limb. Surgery 74:637-640, 1973 .

25. Brandestini M.A. and Forster F.K.: Blood flow imaging using a discrete-fime frequency meter. IEEE Cat. chapter 1344,1978 .

26. Brandestini M.A.: Topoflow -. a digital fiull range Doppler velocity meter. IEEE Tratts on sonics and ultrawonics Vol. Su 25: $287-293,1978$. 
27. Brew W.K.M., FitzGerald D.F.: Transcutaneous assessment of arterial elasticity. OMrasound Med. Brol. 2: $263-270,1976$.

28. Brewster D.C., Darling R.C.: Optimal methods of aortolliac reconstruction. Surgery $84: 739-748,1978$.

29. Bruins Slot H., Strijbosch L., Greep J.M.: Interobserver wariability in single-plane aorto-arteriograplyy. Swrgery, acoepted for publication, 1981 .

30. Bruins Slot H., Breslau P.J., Creep J.M.: A comparative study of strain gauge plethysmography and Doppler ultrasound in patients with acclusiwe arterial disease of the lower extremities. Angiology, actepted for publication 1981 .

31. Bruigninckx C.M.A.: Pre- en postoperatreve emahatie van clandicatio intermattens met behulp man aen Doppler-fowmeter. Thesis, Eindhoven, 1976.

32. Buda J.A., Weber C.J., MCAllister F.F., Voorhees A.B. Factors influencing patency of femoropopliteal artitery bypass grafts. $A m$. J. Surg. $132: 8-12,1976$.

33. Busse R., Pasch T.: Arterielle Strompulsformen - Entstehung und transkutane Registrierumg. In: Utraschall-Doppler-Diagnostik in der Angiologie. Ed. Kriessmann A. und Bollinger A. Thieme Verlag, Stuttgart 1979 .

34. Carter S.A.: Response of ankle systolic pressure to leg exercise in mild or questionable arterial disesso. N. Engt. J. Med. 287: 578-582, 1972 .

35. Canter S.A.: Clinical measurement of systolic pressures in limbs with arterial occlusive disease. IAMA $207: 1869-1874,1969$.

36. Chamberlain $J$, Housley E, Macpherson A.IS.: The relationship between ultrasound assessment and angiography in occlusive arterial disease of the lower limb. Br. J. Surg. 62:64467, 1975.

37. Charlesworth D., Harris P.L., Cave F.D., Tay lor L. A.: Undetected aorto-iliac insurficiency" a reason for early failure of sapenous vein bypass grafts for obstruction of the superficial femoral artery. Br. $f$ Sirg. 62: $567-570,1975$.

38. Chidi C.C., DePalma R.G.: Atherogenic potential of the embolectomy cutheter. Surgery $83 ; 549-557$. 1978 .

39. Chikos P.M., Fischer $\mathbb{L}_{\text {, }}$, Hirsch J., Harley J.D., Thiele B.L., Strandness D. E. : Observer variability in evaluating extracranial carotid artery stenosis. Subjected for publication.

40. Colt J.D.: New Doppler pressure indices plotted as curves. Curve configuration used to determine sites of arterial obstruction. Am. J. Surg. 136:198-201, 1978.

41. Cutler B.S., Thompson I.E., Kleinsasser L.J.y Hempel G.K.: Autologous saphenous vein femoropoptiteal bypass: analysis of 298 cases. Surgery $79: 325-331,1976$.

42. David T.E., Drezner A.D.: Extended profundoplasty for limb salvage. Surgery 84: 758-763,1978.

43. Dean R.H., Yao J.S.T., Stanton P.E., Bergan J.J.: Prognostic indicators in femoropopliteal reconstructions. Arch. Surg. 110:1287-1293,1975.

44. DeBakey M.E.: The development of vascular surgery. Am. J. Surg. 1. 37: 697-738, 1979.

45. DePalma R.G., Clowes A.W. Interventions in atherosclerosis: a review for surgeons. Surgery 84: $175-189,1978$

46. Detre K.M., Wright P.H.E., Murphy M.L., Takaro T.: Observer agreement in evaluating coronary angiograms. Circulation 52: $979-986,1975$.

47. DeWeese J.A.: Anastomotic intimal hyperplasia. In: Vascular grafis, ed. Sawyer P.N., Appleton-CenturyCrofts, New York 1978.

48. Dobrin P.B.: Mechanical properties of arteries. Physiol. Rev. 58: 397-460, 1978.

49. Doriot P.A., Casty M., Milakara B., Anliker M., Bollinger A., Siegenthaler W.: Quantitative amalysis of flow conditions in simulated vessels and large human arteries and veins by moans of ult rasoumd. Ext. Med. Int. Congress Series, no. 363,1975.

50. Dos Santos R., Lamas A.C., Pereira-Caldan J. Arteriografia da aorta e dos vasos abdominais. Med. Contemp. $47: 93,1929$.

5\|. Eastcott H.H.G.: Conservatism in vascular surgery: a mid-atlantic wiew. Am. J Surg. 135:417 420 , 1978.

52. EEans D.H., Quin R.O., Bell P.R.F." The signilicance of blood pressure measurements in patients with peripheral vascular disease. Br. J. Surg. $67: 238-241,1980$.

53. Wvans D.H., Barrie W.W., Asher M.J., Bentley S., Bell P.R.F." The relationship between ultrasonic pulsatility index and proximal arterial stenosis in a canine model. Curc. Rex 46:470-475, 1980.

54. Felix W.R., Sigell B., Gibson R.J., Williams J., Popky G.L., Edelstein A.L., Justin J.R.: Pulsed Doppler ultrasound detection of fllow disturbances in arteriosclerosis. J. Clin. Ultrasound 4: 275-282, 1976 .

55. Fernandes e Fernandes J., Nicolaides A.N.,Angelides N.A., Gordon-Smith I.C.: An objective assessment of common femoral endarterectomy and profundaplasty in patients with superficial femoral occlusion. Situgery 83: $313-318,1978$.

56. FitzGerald D.E., Gosling R.G., and Woodcock J.P.: Grading dymamic capability of arterial collaterul circulation. Lancet 1:66-67, 1971 .

57. FitzGerald D.E and Carr J.: Peripheral arterial disease" assessment by arteriography and alternative moninvasive measurements. Am. J. Roentgenol. 128:385-388, 1977.

58. Franklin D.L., Schlegel W.A., Rushmer R.F.: Blood flow measured by Doppler frequency shluft of back-scattered ultrasound. Science 134: 564-565, 1961. 


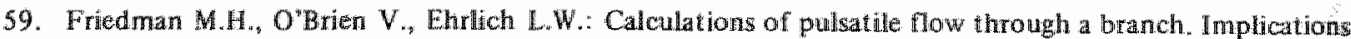
for the hemodynamics of atherogenesis. Circ. Res. 36:277-285, 1975.

60. Fromek A., Coel M., Bernstein E.F.: The importance of combined multigegmentat pressure and Doppler flow velocity studies in the diagnosis of perpheral arterial occlusive disease. Surgery $84: 840-847,1978$.

61. Fry D.L.: Acute vascular endothelial changes associated with increased blood velocity gradients. Circ. Res. 12:165-197, 1968 .

62. Galbratth J.E., Murphy M.L., de Soyza N.: Coronary angiogram interpretation. Interobserwer variability. JAMAL 240: 2053-2056, 1978 .

63. Goodreau J.J., Creagy J.K., Flanigan D.P., Bumham S.J., Kudrna I.C., Schafer M.F., Bergan J.J., Yao I.S.T.: Rational approach to the differentiation of vascular and nevrogenic daudication. Surgery 84: $749.757,1978$.

64. Gosting R.G., King D.H.: Arterial assessment by Doppler-shift ultrasound. Proceedings of the Royal Society of Medicine $67: 447449,1974$

65. Gosling $\mathbb{R}_{*} \mathrm{C}_{\text {, }}$ Newman D.L., Bowden N.L.R., "Twinn, K.W.: The area ratio of normal aortic junctions. Aortic configuration and pulse-wave reflection, Br. J. Radiol. 44:850-853, 1971.

66. Gosling R.G.: Extraction of physiological intormation from spectrum analyzed Doppler-shifted continwous wave ultrasound signals obtained non-inwasively from the arterial system. IEE Med. Electr. 4: $73-125,1976$

67. Gowling R.G. and King D.H.: Contimuous wave ultrasound as an altemative and complement to $x$-rays in vascular examinations. In: Cardionascular applications of ultrasound, Ed. Reneman R., North Holland publishing co., Amsterdam 1974.

68. Gosling R.G. King D. H. and Woodcock J.: Blood-velocity waweforms in the evaluation of atheromatous changes. In: Blood How Measurement. Ed. V.C. Roberts, pp. 33-36, Sector Publishing Ltd. London 1972

68a. Cosling R.G., King D.H.: Uitrasonic angiology. ln: Arteries and veins, Ch. 5, Ed. Harcus and Adamson, Clurchill Livingstone, Lordon 1975.

69. Grosling R.G., King D.H.: Processing arterial Doppler signals for clinical data. In: Handbook of clinical ultrasound, Ed. De Vlieger et al., John Wiley, pp. 613*646, 1978.

70. Gosling R.G., King D.H. Newman D.L., Woodcock J.P.: Transcutaneous measurement of arterial blood-velocity by ultrasound. $J$. Uhrosonics conf. papers: $16-23,1969$.

71. Gribbin B., Steptoe A., Sleight P.: Pulse wave velocity as a measure of blood pressure change. Psychophysiology $13: 86-90,1976$.

72. Haimovici H.: Vascular surgery, principles and techniques. McGraw-Hill Book Company, New York 1976.

73. Harris P.L., Taylor L.A., Cave F.D., Charlesworth D.: The relationship between Doppler ultrasound assessment and angiography in occluisive arterial disease of the lower limbs. Surg. Gynecol. Obstet. 138 : $911-914,1974$.

74. Hill D.A., Jamieson C.W.: The results of arterial reconstruction utilizing the profunda femoris artery in the treatment of rest pain and pre-gangrene. Br. J. Surg. 64:359-361, 1977 .

75. Hoeks A.P.G., Reneman R.S., Ruissen C.J., Smeets F.A.M.: Possibilities and limitations of pulsed Doppler systems. In: Echocardiology, Ed. Lancée T., Pp. 413419, Martínus Nijhoff, The Hague 1979.

76. Hoeks A.P.G., Reneman R.S., Peronneau P.A.: A multigate pulsed Doppler system with serial dataprocessing. IEEE Somics / Ultrasomics, July 1981.

77. Humphries K. N, Hames T.K, Smith S.W.J., Cannon V.A., Chant A.D.B.: Quavtitative assessment of the common femoral to poplitall arterial segment using contintous wave Doppler ultrasound. Ultrasound Med. Biol. 6: 99-105, 1980.

78. Imparato A.M., Kin G.E., Davidson T, Crowley J.G.: Intermittent claudication: its natural course. Surgery 78: $795-799,1975$.

79. Johnson W.C.: Doppler ankle pressure and reactive hyperemia in the diagnosis of arterial imsufficiency. J. Surg. Res, 18:177-180,1975.

80. Jolnston K.W., Maruzzo B.C., Cobbold R.S.C.: Dopplem methods for quantitative measurement and localization of peripheral arteriall occlusive disease by analysis of the blood flow valocity waveform. Chrosound Med. Biod. 4: 209.223, 1978

81. Johnston K.W.: Systolic slope and other pressure measurements in patients with peripheral vascular disease. Surg. Gynecol. Obstet. 140:249.251, 1975 .

82. Jörning P.J.G.: De wenewze overbmugingsplastiek in het femoro-popliteale traject. Thesis, Masatrich", 1981.

83. Kamimski D.L., Barner H.B., Dorighi J.A., Kaiser G.C., Willman V.L.: Femoropopliteal bypass with reversed autogenous wein. Amn. Surg. 177:232-236, 1973 .

84. Kazamias T.M., Gander M.P., Franklin D.L., Ross J.: Blood pressure measurement with Doppler ultrasonic flowmeter. J. Appl. Physiol. 30: 585-588, 1971 .

85. Kempczinski R.F., Rutherford R.B.: Current status of the vascular diagnostic laboratory. In: Advances in Surgery, vol. 12, Ed. Rob, Yearbook Medicall Publishers, 1978.

86. Knox W.G., Finby N., Moscarella A.A.: Limitations of arteriography in determining operability for fenoropopliteal acclusive disease. Amm. Surg. 161: $509.515,1965$.

87. Kriessmann A., Neiss A., Reipert C., Radler M., Rupp N.: Parameteranalyse bei Ultraschall-Doppler- 
Druckmessung vor und nach ischämie-prowokation. In: Uitraschall-Doppler-Dingnostik in der Amgiologie.

Ed. Kriessmann, A., und Bollinger A. Thieme Verlag, Stuttgart 1979.

88. Kuthan F., Burkhalter A., Baitsch R., Ludlin H., Widmer L.K.: Development of occlusive arterial disease in lower limbs. Arch. Surg. 103: 545-547, 1971.

89. Lallemand R.C., Gosling R.G., and Newman D.L.: Role of the bifurcation in atheromatosis of the abdominal aorta. Surg. Gynecol. Obstet. 137: 987-990, 1973.

90. Leather R.P., Shah D.M., Karmody A.M.: The use of extended profundaplasty in limb salvage. Am. $J$. Surg. 136: 359-362, 1978.

91. Linton R.R., Darling R.C.: Autogenous saphenous vein bypass grafts in femoropopliteal obliterative arterial disease. Surgery $51: 62-73,1962$.

92. Lorentsen E., Hoel B.L., Hol R.: Evaluation of the functional importance of atherosclerotic obliterations in the aorto-iliac artery by pressure/flow measurements. Acta Med. Scand. 191: 399-403, 1972.

93. Loubjerg Hansen P., Cross G., Light L.H.: Beam-angle independent Doppler velocity measurement in superficial vessels. In: Clinical blood flow measurement. Ed. J.Woodcock, Sector Publishing Ltd., London 1976.

94. Lunt M.J.: Accuracy and limitations of the ultrasonic Doppler blood velocimeter and zerocrossing detector. Ultrasound Med. Biol. 2: 1-10, 1975.

95. Martin P., Frawley J.E., Barabas A.P., Rosengarten D.S.: On the surgery of atherosclerosis of the profunda femoris artery. Surgery $71: 182-189,1972$.

96. Martin P., Bouhoutsos J.: The medium term results after profundaplasty. Br. J. Surg. 64: 194-196, 1977.

97. Mavor G.E.: The pattern of occlusion in atheroma of the lower limb arteries. Br. J. Surg. 352-364,1956.

98. Maxwell A.E.: Multivariate analysis in behavioural research. Ed. Bartlett M.S. and Cox D., Chapman and Hall, London 1977.

99. May A.G., DeWeese J.A., Rob C.G.: Hemodynamic effects of arterial stenosis. Surgery 53: $513-524$, 1963.

100. McAllister F.F.: The fate of patients with intermittent claudication managed nonoperatively. Am. $J$. Surg. 132: 593-595, 1976.

101. McCombs P.R.: The physiopathologic characteristics of superficial femoral artery occlusion. Surg. Gynecol. Obstet. 148: 775-784, 1979.

102. McDonald D.A.: Blood flow in arteries. Edward Arnold (publishers) ltd., London, 1974.

103. McLeod IF.D.: A directional Doppler flowmeter. Proc. 7 th Int. Conf. Med. Biol. Engng., pp. 13-14, Stockholm 1967.

104. Miller V.M.: Femoropopliteal bypass graft patency: an analysis of 156 cases. Ann. Surg. 180*35.38, 1974.

105. Modgill V.K., Humphrey C.S., Shoesmith J.H., Kester R.C.: The value of profundaplasty in the management of severe femoro-popliteal occlusion. Br. J. Surg. 64: 362-364, 1977.

106. Mol J.M.F.A.: Doppler-haematotachografisch onderzoek bij cerebrale circulatiestoornissen. Thesis, Maastricht 1973.

107. Moore W.S. and Hall A.D.: Unrecognized aortoiliac stenosis. Arch. Surg. 103: 633-638, 1971.

108. Moore S.: Thromboatherosclerosis in normolipidemic rabbits. A result of continued endothelial damage. Lab. Invest. 29: $478,1973$.

109. Mozersky D.J., Sumner D.S., Strandness D.E.: Disease progression after femoropopliteal surgical procedures. Surg. Gynecol. Obstet. 135: 700-704, 1972.

110. Needham T., Yao J.: Assessment of arterial disease of the leg by ultrasound and plethysmography. In: Blood Jlow measurement. Ed. Roberts C., Sector publishing Ltd., London 1972.

111. Newman D.L., Batten J.R., and Bowden N.L.R.: Partial standing wave formation above an abdominal aortic stenosis. Cardiowasc. Res. $11: 160-166,1977$.

112. Nicolaides A.N., Gordon-Smith I.C., Dayandas J., Eastcott H.H.G.: The value of Doppler blood welocity tracings in the detection of aorto-iliac disease in patients with intermittent claudication. Strigery 80: 774-778, 1976.

113. Nie N.H., Hull C.H., Jenkins J.G., Steinbrenner K., Bent D.H.: Statistical package for the sacial sciences. McGiraw-Hill Book Company, New York, 2nd edition, 1975.

114. O'Donnel, T.F., Pauker S.G., Callow A.D., Kelly J.J., McBride K;J., Korwin,S.: The relative value of carotid noninvasive testing as determined by receiver operator characteristic curves. Surgery 87: 9.19, 1980 .

115. Oldham II.B.: Claudication: the case for conservatism. J. Roy. Coll. Surg. Edinburgh 9: 179-193, 1963.

116. O'Rourke M.F., Blazek J.V., Morreels C.L., Krovetz L.3.: Pressure wave transmission along the human aorta. Changes with age and in arterial degenerative disease. Circ. Res. 13:567-579, 1968.

117. O'Rourke M.F., Avolio A.P.: Pulsatile flow and pressure in human systemic arteries. Studies in man and in a multibranched model of the human systemic arterial tree. Circ. Res. 46: 363-372, 1980.

118. Peronneau P.A., Hinglais J.R., Xhaard M., Delouche P., Philippo J.: The effects of curvature and stenosis on pulsatile flow in vivo and in vitto. In: Cardiovascular applications of ultrasound. Ed. Reneman $\mathrm{R}$, North-Holland Publishing Co., Amsterdam 1974. 
119. Poppers P.J, Epstein R.M., Donham R.T." Automatic ultrasound monitoring of blood pressure durimg induced hypotension. Anesthesiology $35: 431-435,1971$.

120. Ray P.S. Lape C.P., Lutes C.A., Dillihunt R.: Hemoropopliteal saphenous wein bypass grafts. Analysis of 150 casces. Am, J. Sirg. $119: 385-391,1970$.

121. Reichle F.A, Shuman C.R., Tyson R.R.: Femorotibial bypass in the diabetic patient for salvage of the lschemic lower extremity. Am. Nourg. 129:603-605, 1975

122. Reid J.M., Davis D.L., Ricketts H.J., Spencer M.P.: A new Doppler flowmeter system and its operation with catheter mounted transducers. In: Cardiovascular applications of ultrasound. Ed. Reneman, $\mathbb{R}_{\text {, }}$ North-Holland Pblishing co., Amsterdam 1974.

123. Reneman R.S., Spencer M.P.: Difficulties ir processing of an analogue Doppler fow signal; with special werence to zerowcrossing meters and quantification. In: Cardiovascular applicalions of ultrasound. Ed. Reneman R, North-Holland Publishing Co, Amsterdam 1974.

124. Reneman R.S. Spencer M.P.:Local Doppler audio spectra in normal and stenosed carotid arteries in man. Utrasound in Med. and Biol. $5: 1-11,1979$.

125. Reneman $\mathbb{R}$.S. Hoeks A.P.G., Bruins Slot H., van Merode T.: The on-lime recording of velocity profiles and its potential in the diagnosis of peripheral arterial lesions. In: Non-invasive cardiovascular diagnosis III. Ed. Dietrich, PSC Publishing Company, Littleton. In press 1981.

126. Reneman R.S.: What measurements are necessary for adequate evaluation of the peripheral arterial circulation, Cardiovasc. Dis. In press, 1981.

127. Rememan R.S., Clarke H.F. Simmons N., Spencer M.P.: In wivo comparison of electromagnetic and Doppler flowmeters; with special attention to the processing of an analogue Doppler low signal. Cardiovasic. Res. $7: 557-566,1973$.

128. Rittenhouse E.A., Maixner W., Burr J.W., Barnes, R.W. Directional arterial flow velocily : a sensitive index of changes in peripheral vascular nesistance. Surgery $79: 350-355,1976$.

129. Roewros J.M.J.G.: Analogue processing of C.W.Doppler flowmeter signals to determine a verage frequency shift momentaneously without the use of a wave analyser. In: Cardiovascular Appications of Utrasound. Ed. Reneman $R$, North-Holland Publishing Co., Amsterdam 1974.

1.30, Ross R., Glomset J., Harker L.: Response to injury and atherogenesis. Am. J. Pathot. 86: 675-684, 1977 .

131. Royster T.S., Lynn R., Mulcare R.J.: Combined aortollac and femoropopliteal occlusive disease. Surg. Cynecol. Obstet. 143: $949-952,1976$.

132. Rutherford R.B. Vascular Surgery. Ed. Rutherford R. B., Saunders Co., Philadelphia 1977.

133. Rutkow I.M., Zuidema G.D.: "Unnecessary Surgery": An npdate. Surgery 84: 671-678, 1978.

134. Sandmann W., Peronneau P., Schweins $G$., Bournat J., Hinglais J... Turbulenzmessung mit den DoppterUltraschallvorfahren: Fine neue Methode der Qualitatskontrolle in der Arterienchirurgie. In: Ultaschall-Doppler-Diagnostik in der Angiologie. Ed. Kriessmann A. Rnd Bollinger A., Thieme Verlag, Stuttgart 1979.

135. Satomura S., Kaneko, Z.: Ultrasonic blood rheograph. Proceedings of the 3 ra International Conf. on Med. Electromics: 254,1960 .

136. Sehlenker J.D., Wolkoff J.S.: Major amputations after femoropopliteal bypass procedures. Am. J. Surg. $129: 495-499,1975$.

137. Schouten H. J.A.: Measuring pairwise agreement among many observers. Bïom. $J .22: 497-504,1980$.

138. Segeren G.C.H.J.: Design and construction of a wave form analyser for on-line determination of PI and TT from blood velocity signals. Technical University of Eindho ven 1979 .

139. Shoor P.M. Fronek A., Bernstein E. I.: Quantitative transcutaneous arterial velocity measurements with Doppler llow whetors. Arch. Surg. 114:922-928, 1979.

140. Skidmore $R$. Woodcock J.P.: Physiological interpretation of Doppler-shift waweforms - II. Validation of the Laplace Iransform method for characterisation of the common famoral blood - velocity/time wat woritin. Uitrasound Med. Bitol. 6: 219-225, 1980.

141. Skidmore R., Woodcock J.P., Wells P.N.T Bird, D., Baird, R.N.: Physiological interpretation of Dopplershift waveforms - III, Clinical Rosults. Utrasound Med. Biol. 6:227-231, 1980.

142. Smith R.C. Ferrington $\mathrm{C}$., Ruckiey C.V.: Cat muscle technetium clearance and Doppler ankle pressure in patients with intermitiont clatication. $V A S A 6: 236-243,1977$.

143. Spence M.P. and Reid I.M.: Quantitation of carotid stenosis with continuous wave (C-W) Doppler ultrasotund. Stroke 10:326-330, 1979 .

144. Stegall H.F., Kardon M.B., Kemmerer W.T.: Indirect measurement of arterial blood pressure by Doppler ultrasonic sphygmonanometry. J. Appl. Physiol. 25: 793-798, 1968.

145. Stehbens W.E. The role of hemodymamics in the pathogenesis of atheroselerosis. Prog. Cardiovasc. Dis. $18: 89-103,1975$

146. Stipa S., Wheelock F.C.: A comparison of femoral artery grafts in diabetic and nondiabetic patients. Am. Jurg. 121: 223-228, 1971 .

147. Strandness $D_{1} L_{\text {. }}$ Bell J.W: An evaluation of the hemodynamic response of the claudicating extremity to oxercise. Surg. Gynecol Obstet. $1237-1242,1964$.

148. Strandness D. C. Introduction: Critical appraisal of the fifteen chapters. In: Methods in angiology, Ed. Verstrate M., Martinus Nifhoff, The Hague 1980.

149. Sumdness D.E., Sumner D.S.: Hemodymamics for surgeons. Grume and Stratton, Now York 1975. 
150. Sumner D.S., Strandmess D.E.: Aurtoiliac reconstruction in patients with combined ilide and superfictol femonal artery occlusion. Surgery $84: 348-355,1978$.

151. Sumner D.S., Strandness D.E.: The relationship between calf blood low and ankle blood pressure in patients with iniermittent claudication. Surgery $65: 763-771,1969$.

152. Szilagyi D.E., Elliott J.P., Hageman J.H. Smith R.F., Dall Omo C.A.. Biologic fate of autogenous vein implants as arterial substitutex: clinica!, angiographic and histopathologic observations in fomoropopliteal operations for atherosclerosis, Ant. Sturg. 178: 232-246, 1973.

153. Tatsuoka M.M.: Discriminant analysis, the study of group differences. In: Selected topics ant aduced statistics. An elementary approach, nr. 6, 1970.

154. Taylor KJ.W., Atkinson $P_{\text {s }}$ de Graff C.S., Dembner A.G., Rosenfield A.T.: Clinical evaluation of pulse - Doppler device linked to Gray scale B-scan equipment. Radiology 129: 745-749, 1978.

155. Thompson B.W. Read R.C., Slayden JiE., Boyd C.M. The role of primary and secondary profundaplasty in the treatment of vascular insufficiency. J. Cardiovasc. Surg. 1.8:55-62, 1977.

156. Thompson I.E., Garret W.V.: Peripheral-arterial surgery, N. Engl J. Med. 302; 491-503, 1980.

157. Thulesius 0 ., Gjöres J.E.: Use of Doppler shift detection for determining peripheral arterial blood pressure. Angiology 22:594-603, 1971.

1158. Thulesius O.: Simultane Doppler-sonographie von Arm und Beingefâssen bei atteriellen Okklusionen. In: Uhraschall-Dopplep-Diagnostik in der Angiologie, Ed. Kriesmann A. und Bollinger A. Thieme Verlag, Stutigart 1979.

159. Van den Bos G.C., Westerhol N., Elzinga G., Sipkema P.: Rethection in the systemic arterial system : effects of aortic and carotid occlusion. Cardiovasc. Res. $10: 565-573,1976$.

160. Vam de Water J.M., Laska E.D., Ciniero W. W.: Patient and operation selectivity. The peripheral vascuhar laboratory. Arn. Surg. 189: 143-146,1979.

161. Verstraete M.: Guidelines for clinical trials set up to determine the ugefulness of drugs in patients with symptoms ascribed to decreased flow in a particular vascular area. In: Methods in Angiology. Ed.

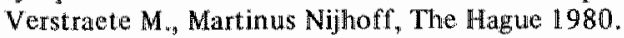

162. Waibel P.P., Wolff $G_{. .}$: The collateral circulation in occlusions of the femorall artery." an experimental study. Surgery 60:912-918, 1966.

163. Ward A.S., Morris-Jones W.: The long term results of profundaplasty in femoropopliteal artertal occlusion. Br. J. Surg. 64: 365-367,1977.

164. Warren R., Gomez R.L., Marston J.A.P., Cox J.S.T." Femoropopliteal arteriosclerosis oblitertans arterigraphic patterns and rates of progression. Surgery $55: 135-143,1964$.

165. Weber R., Becker H.M., Baumann G.: Die profundarevaskularisation. The revascularization of the profunda femoris artery. Thoraxchinurgie 25: 132-138, Thieme 1977.

166. Welsh P., Repetto R.: Revascularization of the profunda femoris artery in aortoliac occlusive disease. Surgery $78: 389-393,1975$.

167. Wesolowski S.A., Fries C.C., Sabini A.M., Sawyer P.N.: The significance of turbulence in hentic systems and in the distribution of the atherosclerotic lesion. Surgery $57: 155-162,1965$.

168. Widmer L.K., Greensher A., Kannel W.B.; Occlusion of peripheral arteries. A study of 6.400 working subjects. Circulation 30: 836-842, 1964.

169. Winsor T.: Influence of arterial disease on the systolic blood pressure gradients of the extremity. Am. $J_{0}$ Med. Sc. 220:117-126, 1950 .

170. Woodcock J.P., Gosling R.G., FitzGerald D.E.: A new non-invasive technique for assessment of superficial femoral artery obstruction. Bt. J. Surg. 59: 226-231, 1972.

171. Wylie E.J.: In: Barker W.F., Crawford E.S., Mannick J.A., Wylle E.J. : The current status of fernoro. popliteal bypass for atherosclenotic occlusive disease: al panel discussion. Surgery 79: 30-36, 1976.

172. Yao S.T. Hobbs J.T., Irvine W.T.: Ankle systollc pressure measurements in arterial disease affocting the lower extremities. Br. J. Surg. 56:676-679, 1969.

173. Yao S.T. Needham T.N. Gourmoos C. Irwine W.T.: A companative study of straingauge plethysmography and Doppler ultrasound in the assessment of occlusive arterial disease of the lower extremitics. Surgery $71: 4-9,1972$.

174. Yao S.T.: Haemodynamic studies in peripheral arterial discase. Br. J. Sitg. 57: 761-766, 1970.

175. Youmg D.F., Cholvin N.R., Roth A.C.: Pressure drop sacross artificially induced stenoses in the femoral. arteries of dogs. Circ. Res. $36: 735-743,1975$.

176. Zir L.M., Miller S.W., Dinsmore R.E., Gilbert J.P., Harthorne J.W.: Interobserver variability in coronary angiography. Circulation 53: 627-632, 1976. 


\section{Acknowledgements}

With gratitude I acknowledge the support of a great many people in Maastricht and other parts of the world.

Looking back upon the years of my surgical training in Rotterdam I feel greatly indebted to the late Prof. Dr. H. Muller and to Prof. Dr. H. van Houten, for encouraging my budding interest in the hemodynamics of vascular surgery.

It was through my friend Hans Jeekel that I met Prof. Dr. J.M. Greep, who provided me with the opportunity to start a vascular laboratory in Maastricht, in which the material for this study was collected. The enthusiasm and never failing patience of Renée Nicolaes, who performed most of the noninvasive measurements, deserves special attention; she, together with Monique Kupers, who skillfully helped creating the many illustrations, and Annie van Bun, made the vascular laboratory of St. Annadal Hospital a fine place to work in.

I owe a great deal to Arnold Hoeks, Cees Ruissen and Rob Reneman, their stimulating and sometimes mind-bending conversations have been of invaluable help.

The constructive attitude of Leo Strijbosch and Lex Volovics, who gave me the benefit of their statistical knowledge, contributed much to this study.

Special thanks must be reserved for Prof. Dr. R.S. Reneman, who has been a wonderful 'director', advising and correcting me in a highly efficient manner.

I also would like to express my deep appreciation towards Prof. Gene Strandness and Prof. Ray Gosling for their efforts as external examiners of this thesis.

I feel grateful to Prof. Dr. J.M. Greep and Prof. Dr. H.A.J. Lemmens for their critical contributions to this work, and to Dr. B. Janevski for his splendid collaboration in the field of arteriography.

Through the priceless work of Gerda Haemers, with her proverbial skill at the IBM6, this thesis found its way to the printer for which I thank her.

My deepest gratitude concerns my parents who, among other things, will feel that their son at last finished his studies, and without whose always tactful advice and continuous sympathy this thesis would never have been written.

Last but not least I would like to mention Liesbet, Bartje and Annemaria for 'being there' in my moments of stress, and for putting up with me these past hectic months.

\section{Curriculum vitae}

Harm Bruins Slot was born on the 16th of April, 1942 in Leiden, the Netherlands. After graduating from high school in Rotterdam he studied at the Medical Faculty in Leiden from' 1959 to 1967. Before starting his surgical training he spent three years in Malawi, working in a mission hospital. From 1971 to 1977 he was a resident in surgery in the University Hospital Dijkzigt, Rotterdam (head: the late Prof. Dr. H. Muller). Since January 1977 he works as 'chef de clinique' at the surgical department of the St. Annadal Hospital, Maastricht (head: Prof. Dr. J.M. Greep). 
